\title{
Thermodynamic and spectral properties of quantum many-particle systems
}

\author{
Dissertation \\ zur Erlangung des mathematisch-naturwissenschaftlichen \\ Doktorgrades \\ „Doctor rerum naturalium“ \\ der Georg-August-Universität Göttingen
}

vorgelegt von

Sebastian Fuchs

aus Lippstadt

Göttingen, 2011 
Referent:

Korreferent:

Tag der mündlichen Prüfung: 21.1.2011
Prof. Dr. Thomas Pruschke

Prof. Dr. Fakher F. Assaad 
On peut même dire, à parler en rigueur, que presque toutes nos connaissances ne sont que probables; et dans le petit nombre des choses que nous pouvons savoir avec certitude, dans les sciences mathématiques elles-mêmes, les principaux moyens de parvenir à la verité, l'induction et l'analogie se fondent sur les probabilités; [...]

Pierre-Simon De LAPlace ${ }^{[100]}$ 


\section{Contents}

1 Introduction 1

2 The Hubbard model 5

2.1 The Hubbard model in condensed-matter physics . . . . . . . . . . 5

2.1.1 Antiferromagnetism . . . . . . . . . . . . . 8

2.1.2 Mott-Hubbard metal-insulator transition . . . . . . . . . . . 10

2.1.3 Magnetic frustration . . . . . . . . . . . . . . . 11

2.2 Cold atoms in optical lattices . . . . . . . . . . . . . . . 13

2.2.1 Theoretical modeling . . . . . . . . . . . . . . . . 14

3 Dynamical cluster approximation 17

3.1 Cluster structure . . . . . . . . . . . . . . . . . . 18

3.2 Outline of the method . . . . . . . . . . . . . . . 20

3.3 Diagrammatic motivation . . . . . . . . . . . . . . . 22

3.4 Effective cluster model . . . . . . . . . . . . . . . . . . . 24

3.5 Antiferromagnetic order . . . . . . . . . . . . . . 26

3.6 Finite-size scaling . . . . . . . . . . . . . . . . . . . . . . 28

3.7 Cluster selection . . . . . . . . . . . . . . . . . . . . . . . 29

3.7.1 Topological imperfection . . . . . . . . . . . . . . . . . 29

3.7.2 Geometry and symmetry . . . . . . . . . . . . . . 31

3.8 Summary . . . . . . . . . . . . . . . . . 33

4 Quantum Monte Carlo simulations in continuous time 35

4.1 Monte Carlo evaluation . . . . . . . . . . . . . . . . . . 36

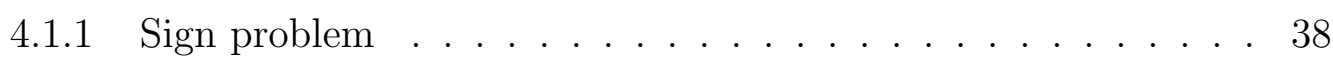

4.2 Continuous-time interaction expansion . . . . . . . . . . . . . . . 39

4.2.1 Monte Carlo updates . . . . . . . . . . . . . . . . . . . 41

4.2 .2 Observables . . . . . . . . . . . . . . . . 42

4.3 Continuous-time auxiliary-field method . . . . . . . . . . . . 45

4.3.1 Monte Carlo updates . . . . . . . . . . . . . . . . . . . 46

4.3 .2 Observables . . . . . . . . . . . . . . . . . 46

4.4 Summary . . . . . . . . . . . . . . . . . . . 47

5 Thermodynamics of the 3D Hubbard model on approach to the Néel transition 
5.1 Method ........................ 49

5.2 Results at half filling . . . . . . . . . . . . . . . . . . 52

5.3 Results away from half filling . . . . . . . . . . . . . 54

5.4 Entropy in the optical lattice system . . . . . . . . . . . . 57

5.5 Summary . . . . . . . . . . . . . . . . . 58

6 Analytic continuation of quantum Monte Carlo data $\quad 61$

6.1 Analytic continuation . . . . . . . . . . . . . . . 62

6.2 Maximum entropy method . . . . . . . . . . . . . . . . . . . 64

6.2.1 Bayesian statistical inference . . . . . . . . . . . . . 64

6.2.2 Bayesian inference and the regularization parameter $\alpha$. . . 65

6.3 Stochastic analytical inference . . . . . . . . . . . . . . . 66

6.3.1 Bayesian statistical inference . . . . . . . . . . . . . . 68

6.3.2 Bayesian inference and the regularization parameter $\alpha$. . . 69

6.4 Monte Carlo evaluation . . . . . . . . . . . . . . . . . . . . . 70

6.5 Simulation results . . . . . . . . . . . . . . . . . 70

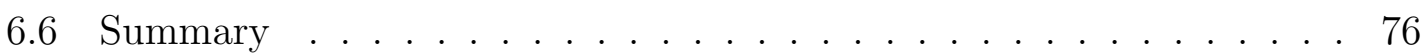

7 Spectral properties of the 3D Hubbard model $\quad 79$

7.1 Method ........................ 79

7.2 Properties of the paramagnetic phase . . . . . . . . . . . 81

7.2 .1 Metallic phase . . . . . . . . . . . . . . . . 82

7.2 .2 Mott-Hubbard metal-insulator transition . . . . . . . . . 86

7.3 Antiferromagnetic phase . . . . . . . . . . . . . . . 90

7.4 Summary . . . . . . . . . . . . . . . . . . . 94

8 Summary $\quad 97$

$\begin{array}{ll}\text { A CT-INT: Inversion by partitioning } & 99\end{array}$

$\begin{array}{ll}\text { B High-frequency expansion } & 101\end{array}$

$\begin{array}{lr}\text { C Sub-matrix updates for the CT-AUX algorithm } & \mathbf{1 0 5}\end{array}$

C.1 Sub-matrix updates . . . . . . . . . . . . . . . . . . . 105

C.2 Monte Carlo updates . . . . . . . . . . . . . . . . . . . . 107

C.3 Summary . . . . . . . . . . . . . . . . . . . . . . . . 109

D Analytic continuation: Implementation details $\quad \mathbf{1 1 1}$

D.1 Maximum entropy algorithm . . . . . . . . . . . . . . . . . . . 112

D.2 Stochastic analytical inference . . . . . . . . . . . . . . 113

D.2.1 Wang-Landau algorithm . . . . . . . . . . . . . . 115

$\begin{array}{ll}\text { E Cluster table } & 117\end{array}$ 


\section{Introduction}

The investigation of quantum many-particle systems is a central goal of modern condensed-matter physics. Complex physical phenomena such as high-temperature superconductivity [101], heavy-fermion behavior [72], and correlation-driven metalinsulator transitions [78] are central aspects of this particular field of science.

The Hubbard model $[76,63,91]$ is a paradigm of correlated-electron physics. The model describes interactions between electrons in the most basic way by assuming the Coulomb repulsion to be local to each lattice site. It is one of the simplest quantum-mechanical lattice models capable of capturing relevant physical aspects of strongly correlated electron systems such as high-temperature superconductivity [5] or correlation-driven insulators [78]. Outside of condensed-matter physics, the Hubbard model plays an important role in the explanation of many-body phenomena observed in ultra-cold atoms trapped in optical lattices [23]. These systems present a remarkably pure realization of the Hubbard model and other quantum-mechanical lattice models [80]. In contrast to condensed-matter systems, the model parameters can be precisely controlled offering the possibility of a systematic experimental study of models that were initially formulated for applications in condensed-matter physics.

Despite its simple structure, the Hubbard model cannot be solved in general. Especially for lattices in two and three dimensions no exact solution exists. Therefore, numerical methods are of prime importance of the investigation of its properties. In this thesis, we study the three-dimensional (3D) Hubbard model using quantum Monte Carlo (QMC) simulations. Our aim is a precise numerical study of its properties in the thermodynamic limit.

QMC algorithms are powerful tools to calculate thermodynamic properties of interacting quantum many-particle systems, such as spin models [66, 158, 159] or more generally bosonic systems $[68,162]$. However, a direct investigation of lattice models in the thermodynamic limit is generally impossible because QMC simulations cannot treat infinite lattice sizes. A reliable finite size extrapolation to the thermodynamic limit is very difficult, especially for fermionic systems such as the Hubbard model, where the sign problem [184] limits simulations to either very small systems or to special cases such as particle-hole symmetric models.

Therefore, approximative schemes that allow calculations directly in the thermodynamic limit are advantageous. A widely applied approximation of this kind is the dynamical mean-field theory (DMFT) $[48,96]$. The DMFT maps the infinite-lattice problem onto a single-site impurity model embedded in a mean field. The single- 
site problem is then solved by QMC or other suitable impurity solvers like exact diagonalization methods [30] or Wilson's numerical renormalization group (NRG) algorithm [27]. The DMFT approximates all quantum fluctuations to be local and treats long ranged correlations in a mean-field limit only, an approximation that is only exact in the limit of infinite dimensions $[118,126]$. The neglect of non-local many-body correlations renders the DMFT incapable of capturing physical effects such as spin-wave and spin-liquid physics or disorder-induced localization.

Cluster mean-field theories [109] represent a systematic extension of the DMFT by expanding the single impurity to a finite cluster embedded in a mean field. By systematically enlarging the cluster size, more and more non-local correlations are re-introduced into the problem. The original lattice problem is regained in the limit of infinite cluster size. Thus, cluster mean-field theories offer the possibility of a systematic finite-size analysis. Two distinct cluster mean-field theory algorithms have been developed in recent years: the dynamical cluster approximation (DCA) $[71,70]$ and the cellular dynamical mean-field theory [97]. Since the difference between the two methods is mainly of a technical nature, we use the DCA exclusively for this thesis.

We employ QMC simulations to solve the effective cluster model of the DCA. Alternative methods applied as impurity solvers in the DMFT context such exact diagonalization or NRG are not applicable to the more complex cluster problems. However, even when using QMC methods, the transition from a single impurity to finite clusters dramatically enhances the numerical complexity of the simulation requiring highly optimized algorithms and the application of high-performance computing resources.

The quasi-standard for QMC simulations of fermionic systems used to be the Hirsch-Fye algorithm [75] which suffered from a systematic error caused by an artificial discretization of the imaginary time axis. We use recently developed QMC algorithms $[152,153,59]$ which are formulated in continuous imaginary time and are free of any systematic error. These QMC methods in combination with additional performance-optimized algorithms [56] enable us to do a precise study of the thermodynamic properties of the 3D Hubbard model in the thermodynamic limit.

Important insight can be gained into the properties of correlated materials by studying dynamic quantities such as single-particle spectra. The second focus of this thesis is therefore the calculation of spectral properties of the Hubbard model.

QMC methods map the quantum-mechanical lattice model on a classical one at the expense of an additional dimension, which is identical or at et least closely related to an imaginary time axis [40, 34, 160]. For this reason, QMC algorithms can only provide dynamical data on the imaginary time or frequency axis. The necessary analytic continuation to the physically relevant real axis has proven to be a difficult problem that has to be approached by advanced data analysis tools.

The maximum entropy method (MEM) [83] is the standard technique to handle this problem. It uses methods of Bayesian inference $[86,61]$ to determine the most 
probable spectrum subject to the incomplete information given by the QMC data. We apply the MEM to the problem of extracting momentum-resolved single-particle spectra from QMC simulations of the 3D Hubbard model. Furthermore, we discuss and expand algorithms that were recently proposed as alternatives to the standard MEM approach $[157,13,176,45]$ and provide a full interpretation of these methods within the framework of Bayesian probability theory.

The thesis starts with an introduction to the Hubbard model and its properties and presents its applications in the context of condensed-matter physics and coldatom experiments (chapter 2).

The subsequent two chapters deal with the theoretical and numerical methods which were used to solve the Hubbard model within a cluster approximation. An overview of the DCA as the applied cluster mean-field theory is given in chapter 3, whereas the QMC techniques to solve the resulting cluster problem are described in chapter 4.

As first application of these techniques, chapter 5 presents an expansive study of the thermodynamic properties of the 3D Hubbard model for temperatures above the Néel temperature. To make contact to cold-atom experiments, we focus on thermodynamic properties, that could be valuable to experimental research and compute properties of the model subject to an external trap.

The subject matter of the following two chapters is the calculation of energy spectra. Chapter 6 introduces the MEM as a method used for the necessary analytic continuation of QMC data. Additionally, a detailed characterization of stochastic analytical inference, an alternative to the MEM, and its application to a test case is given. Finally, momentum-resolved energy spectra of the 3D Hubbard model in the paramagnetic and the antiferromagnetic phase are presented in chapter 7 . The thesis concludes with a short summary of the results (chapter 8). 


\section{The Hubbard model}

The single-band Hubbard model $[76,63,91]$ is commonly defined as

$$
H=-t \sum_{\langle i, j\rangle \sigma} c_{i \sigma}^{\dagger} c_{j \sigma}+U \sum_{i} n_{i \uparrow} n_{i \downarrow}
$$

The operators $c_{i \sigma}^{\dagger}\left(c_{i \sigma}\right)$ create (annihilate) an electron with spin $\sigma \in\{\uparrow, \downarrow\}$ at lattice site $i, n_{i \sigma}=c_{i \sigma}^{\dagger} c_{i \sigma}$ is their corresponding number density, $t$ is the hopping parameter between neighboring sites (denoted by $\langle i, j\rangle$ ), and $U$ implements the Coulomb repulsion. Note that Coulomb repulsion is taken to be local at each lattice site. This is characteristic approximation of the Hubbard model.

We study the Hubbard model in three dimensions on a simple-cubic lattice. For $U=0$ eq. (2.1) simplifies to the tight-binding model for non-interacting fermions,

$$
H_{\mathrm{tb}}=-t \sum_{\langle i, j\rangle \sigma} c_{i \sigma}^{\dagger} c_{j \sigma}=\sum_{\boldsymbol{k} \sigma} \epsilon_{\boldsymbol{k}} c_{\boldsymbol{k} \sigma}^{\dagger} c_{\boldsymbol{k} \sigma} .
$$

The second expression follows from a Fourier transformation into momentum space ${ }^{1}$ using the dispersion relation

$$
\epsilon_{\boldsymbol{k}}=-2 t \sum_{i=1}^{3} \cos \left(k_{i}\right)
$$

The momentum $\boldsymbol{k}=\left(k_{1}, k_{2}, k_{3}\right)$ is an element of the first Brillouin zone which - in the case of the simple-cubic lattice - is of cubic shape. The bandwidth $W=12 t$ of this dispersion is used as energy scale for all results in this thesis.

\subsection{The Hubbard model in condensed-matter physics}

Although the repulsion between electrons per se is a strong and long-ranged force, the Coulomb potential is screened by the presence of other electrons resulting in a short ranged Yukawa potential. This effect combined with the high velocity of electrons near the Fermi level cause electron interactions to be a small effect in most

\footnotetext{
${ }^{1}$ We use the convention $\hbar=1$ in all chapters of this thesis. Consequently, we do not distinguish between the terms "wave vector" and "momentum", or "frequency" and "energy", respectively.
} 
materials. In this case, the system can be described by Fermi liquid theory [12], i. e., by quasi-particles that effectively behave like free fermions with renormalized coupling parameters. However, this is not always true and electron-electron correlations can have a significant influence on the physical properties of the material. The Hubbard model is one of the simplest quantum-mechanical lattice model that captures this electron-electron interaction explicitly.

We can motivate the Hubbard model by starting with the general Hamiltonian for interacting electrons subject to the electric potential of the atoms [39]. If we employ the Born-Oppenheimer approximation and assume the atoms to be fixed, we can describe the influence of the atoms by a conservative lattice potential $V_{\mathrm{L}}(\boldsymbol{r})$. The Hamiltonian of electrons of mass $m$, charge $e$, and spin $\sigma$ in second-quantized form is

$$
\begin{aligned}
H= & \sum_{\sigma} \int \mathrm{d} \boldsymbol{r} \psi_{\sigma}^{\dagger}(\boldsymbol{r})\left(\frac{-\hbar^{2}}{2 m} \nabla^{2}+V_{\mathrm{L}}(\boldsymbol{r})\right) \psi_{\sigma}(\boldsymbol{r}) \\
& +\frac{1}{2} \sum_{\sigma \sigma^{\prime}} \int \mathrm{d} \boldsymbol{r} \mathrm{d} \boldsymbol{r}^{\prime} \frac{e^{2}}{\left|\boldsymbol{r}^{\prime}-\boldsymbol{r}\right|} \psi_{\sigma}^{\dagger}(\boldsymbol{r}) \psi_{\sigma^{\prime}}^{\dagger}\left(\boldsymbol{r}^{\prime}\right) \psi_{\sigma^{\prime}}\left(\boldsymbol{r}^{\prime}\right) \psi_{\sigma}(\boldsymbol{r})
\end{aligned}
$$

using the fermionic field operators $\psi_{\sigma}(\boldsymbol{r})$. An expansion in localized Wannier states $W_{\alpha}\left(\boldsymbol{r}-\boldsymbol{r}_{i}\right)$,

$$
\psi_{\sigma}(\boldsymbol{r})=\sum_{i \alpha} c_{i \alpha \sigma} W_{\alpha}\left(\boldsymbol{r}-\boldsymbol{r}_{i}\right)
$$

with band index $\alpha$ introduces the annihilation (creation) operators $c_{i \alpha \sigma}^{(\dagger)}$ at each lattice site. The result of the expansion is

$$
H=-\sum_{i j \alpha \sigma} t_{i j}^{\alpha} c_{i \alpha \sigma}^{\dagger} c_{j \alpha \sigma}+\frac{1}{2} \sum_{i j k l \sigma \sigma^{\prime}} \sum_{\alpha \beta \gamma \delta} V_{i j k l}^{\alpha \beta \gamma \delta} c_{i \alpha \sigma}^{\dagger} c_{k \beta \sigma^{\prime}}^{\dagger} c_{l \gamma \sigma^{\prime}} c_{j \delta \sigma}
$$

with

$$
V_{i j k l}^{\alpha \beta \gamma \delta}=\int \mathrm{d} \boldsymbol{r} \mathrm{d} \boldsymbol{r}^{\prime} \frac{e^{2}}{\left|\boldsymbol{r}^{\prime}-\boldsymbol{r}\right|} W_{\alpha}^{*}\left(\boldsymbol{r}-\boldsymbol{r}_{i}\right) W_{\beta}^{*}\left(\boldsymbol{r}^{\prime}-\boldsymbol{r}_{j}\right) W_{\gamma}\left(\boldsymbol{r}^{\prime}-\boldsymbol{r}_{k}\right) W_{\delta}\left(\boldsymbol{r}-\boldsymbol{r}_{l}\right)
$$

and the hopping

$$
t_{i j}^{\alpha}=\int \mathrm{d} \boldsymbol{r} W_{\alpha}^{*}\left(\boldsymbol{r}-\boldsymbol{r}_{i}\right)\left(-\frac{\hbar^{2}}{2 m} \nabla^{2}+V_{\mathrm{L}}(\boldsymbol{r})\right) W_{\alpha}\left(\boldsymbol{r}-\boldsymbol{r}_{j}\right)
$$

Now we employ the approximation that only one band is relevant for the physical properties of the systems. This approximation is justified if the inter-band interactions are small and all bands except one conduction band are far away from the Fermi level. The single-band Hubbard model eq. (2.1) then follows from eq. (2.6) 


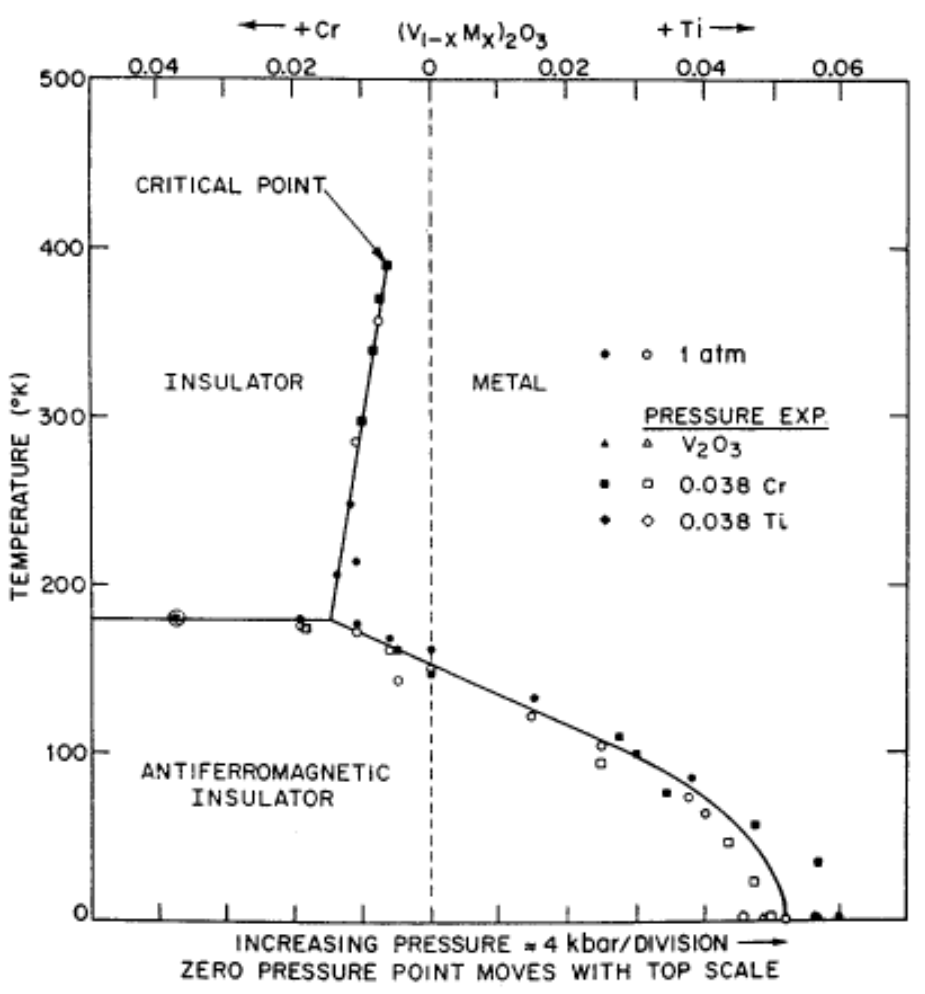

Figure 2.1: (taken from ref. [48]) Experimental phase diagram of $\mathrm{V}_{2} \mathrm{O}_{3}$ as measured by MCWhan et al. [115] as function of doping or as a function of pressure.

if we keep only the most significant terms, i. e., the hopping between nearest neighbors and the local coulomb repulsion $U=\frac{1}{2} V_{i i i i}^{\alpha \alpha \alpha \alpha}$. The second approximation is justified if the intra-atomic Coulomb repulsion is large compared to the inter-atomic interaction and, at the same time, cannot be neglected compared to the hopping.

This situation is believed to be realized in many materials such as transition metal oxides, cuprates, actinides, or materials containing rare-earth elements [78]. These materials often feature flat conduction bands consisting mainly of $d$ and $f$ orbitals, whose strong localization causes a dominant local Coulomb repulsion between electrons in the same orbital. These materials exhibit prominent evidence of strong electron-electron correlations including various types of magnetism, correlationdriven metal-insulator transitions, or superconductivity.

An example for a 3D transition metal oxide is vanadium(III) oxide, $\mathrm{V}_{2} \mathrm{O}_{3}$. Fig. 2.1 shows the phase diagram of this compound as a function of pressure and as function of doping with chromium or titanium. The increase of pressure causes a larger overlap between orbitals and thus enlarges the hopping $t$, or, alternatively, decreases the fraction $U / t$. The doping with $\mathrm{Cr}^{3+}$ or $\mathrm{Ti}^{3+}$ ions has a similar effect $[115,78]$. The phase diagram exhibits an antiferromagnetic insulator at low temperature. A transition line emerges from this phase which separates a metallic and an insulating 
paramagnet. The transition line ends at a critical point at larger temperatures, above which only a crossover between metallic and insulating behavior is observed. We will show in the subsequent two sections that the Hubbard model provides the possibility to study both metal-insulator transitions and antiferromagnetism.

The Hubbard model is one of the simplest quantum-mechanical model of interacting electrons capable of capturing - at least qualitatively - basic physical properties of strongly correlated materials. For a quantitative description of real materials, the precise orbital structure and lattice configuration must often be taken into account. This leads to more complicated forms of the kinetic energy and Coulomb repulsion. One can try to incorporate these effects by extending the Hubbard model to include, for example, multiple bands [178] or non-local interactions [185]. But even in its most basic formulation - despite its very simple structure - the Hubbard model can only be solved exactly in one [39] and infinite spatial dimensions [48].

In one dimension, the Hubbard model can be solved in most cases by the Bethe ansatz $[103,104]$. Here even weak Coulomb interactions cause strong perturbations and the physical features are distinctly different from systems in higher dimensions [39].

In the other exactly solvable limit, i. e., $D \rightarrow \infty$, it turns out that all dynamical renormalizations become purely local $[118,126]$. As it can be related to a genuine mean-field theory, the name dynamical mean-field theory (DMFT) has been coined for this solution. Besides being exact in the limit $D \rightarrow \infty$, it is frequently used to approximately treat two respectively three-dimensional systems. Within DMFT, the phase diagram of the single-band Hubbard model eq. (2.1) is already well understood. It features an antiferromagnetic phase at half filling, which will be discussed in the next section. Antiferromagnetism is persistant off half filling up to a critical doping and shows additional phase separation [185, 85, 202]. For very large values of $U$ and low temperatures, antiferromagnetism is replaced by a small ferromagnetic phase off half filling [127, 131, 136].

To what extend these results are transferable to $2 \mathrm{D}$ and $3 \mathrm{D}$ systems is still an open question. Especially, the 2D Hubbard model is studied intensely, since it is believed to capture the physics of the superconducting planes in high-temperature superconductors [200, 5, 109]. In the following, we will focus on results for the 3D Hubbard model, especially on the two mechanism we are mostly interested in, antiferromagnetism and the Mott-Hubbard metal-insulator transition.

\subsubsection{Antiferromagnetism}

The Hubbard model eq. (2.1) at half filling shows antiferromagnetic order at finite temperature for any value of $U>0$. Antiferromagnetic order breaks the translational symmetry of the lattice causing a doubling of the unit cell. In momentum space, this translates to a reduction of the first Brillouin zone. The resulting antiferromagnetic Brillouin zone possesses only half of the original volume (see fig. 2.2a). 

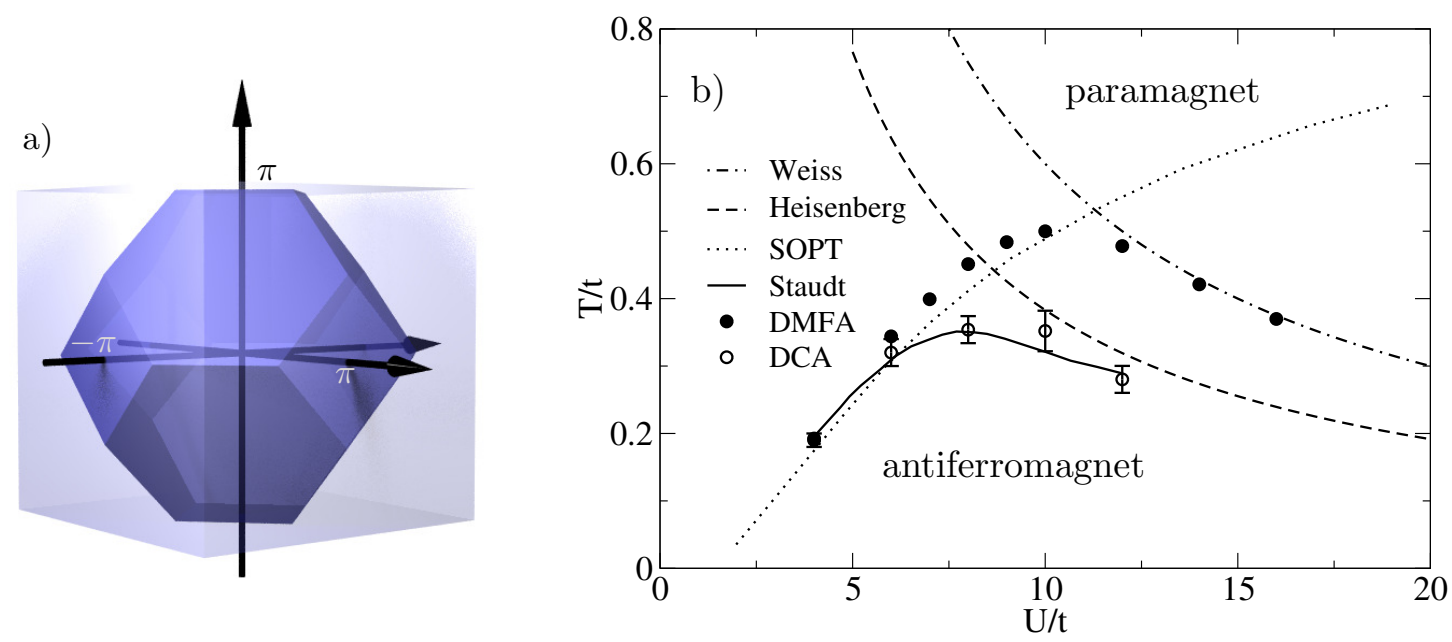

Figure 2.2: Panel a: the reduced first Brillouin zone of the simple-cubic lattice with antiferromagnetic order. Panel b (taken from ref. [92]): the antiferromagnetic phase diagram of the $3 \mathrm{D}$ Hubbard model at half filling as calculated by lattice QMC [144] (denoted by "Staudt"), second order perturbation theory (SOPT) [179], the Heisenberg model [154] Weiss mean-field theory ("Weiss"), DMFT (denoted by "DMFA"), and DCA [92]. Note that the units refer to the hopping parameter $t$.

Following an argument by SLATER [170], the back-folding of the conduction band into the reduced Brillouin zone combined with the opening of a gap at the new zone boundary causes the ordered state to be an insulator. This insulating behavior is primarily induced by symmetry breaking and already exists at small interaction strengths. It is not related to the correlation driven insulators discussed in section 2.1.2.

At very large $U$, on the other hand, it is energetically highly disadvantageous that two electrons occupy the same site. If we consider the model at half filling, this implies that there is precisely one electron per site. No electron can move and the system is insulating. In this limit, the remaining spin degrees of freedom can be accurately described by the Heisenberg model [47] whose antiferromagnetic phase is well studied [154].

The extent of the antiferromagnetic phase between these two limiting cases can be determined by QMC simulations. Fig. 2.2b displays the antiferromagnetic phase diagram of the 3D Hubbard model at half filling as calculated by second order perturbation theory [179], the Heisenberg model [154], QMC on finite lattices [144], Weiss mean-field theory, DMFT, and dynamical cluster approximation (DCA) [92] (cf. chapter 3 for details of the DCA). The figure shows how the DCA and lattice QMC results interpolate between those from perturbation theory at small $U$ and those from the Heisenberg model at large $U$. The DMFT overstates the Néel tem- 
perature already at intermediate values of $U$ and converges with the results of the (static) Weiss mean-field theory [191] at larger interaction values.

\subsubsection{Mott-Hubbard metal-insulator transition}

The Hubbard model also features a correlation-driven metal-insulator transition as first described by Mотт [124]. The argument given in section 2.1.1, that the system is insulating at half filling and for $U \rightarrow \infty$, still holds for the paramagnetic state. For weak interaction, on the other hand, the paramagnetic state can be accurately described by Fermi liquid theory [12] and is therefore a metal like the non-interacting system itself. A potential phase transition between these to states is the Mott-Hubbard metal-insulator transition (MH-MIT).

Unfortunately, the low-temperature behavior of the single-band Hubbard model on the simple cubic lattice is completely dominated by the antiferromagnetic phase. Above the Néel temperature, only a crossover from metallic to insulating behavior can be observed [144] while the whole antiferromagnetic phase is insulating by symmetry. Thus, the MH-MIT cannot be observed directly. However, it is nevertheless possible to study the MH-MIT on the Hubbard model by artificially suppressing antiferromagnetic order in the system. While this may appear unphysical at first glance, it is the proper route to understand the influence of electron correlations in the model, because it also allows to distinguish between effects that come from fluctuations, hence are dynamical, and those that are due to actual symmetry breaking.

Studies of the MH-MIT at finite temperatures were performed for a Bethe lattice in the limit of infinite dimension, i. e., within DMFT [28, 87, 181]. The DMFT, like any other mean-field theory, offers the possibility to artificially suppress antiferromagnetic order. Results calculated by the numerical renormalization group method (NRG) [27, 28], QMC [87], and the so-called iterated perturbation theory (ITP) [150] are presented in fig. 2.3. Note that the units of the graph refer to the bandwidth $W_{\text {Bethe }}$ of the Bethe lattice. The ITP employs only second-order perturbation theory to solve the local problem and its results differ notably from those of the other two methods. The DMFT exhibits a coexistence region below a critical temperature $^{2} T_{\mathrm{c}} \approx 0.02 W_{\text {Bethe }}$ (according to NRG and QMC). Inside this region, the solution is either metallic or insulating depending on the starting point of the DMFT iteration (cf. section 3.2 for details of the DMFT/DCA algorithm). By comparison of the free energies of the two possible solutions, one can decide which one of the two solutions is actually the physical one. Since estimation of the free energy is particularly difficult for many impurity solvers including QMC (see section 4.1), further DMFT studies with alternative methods that enable the calculation of free energies, e.g., exact diagonalization techniques, show that the actual phase transi-

\footnotetext{
${ }^{2}$ We set Boltzmann's constant $k_{\mathrm{B}}$ to unity in this thesis and will therefore refer to temperatures in energy units.
} 


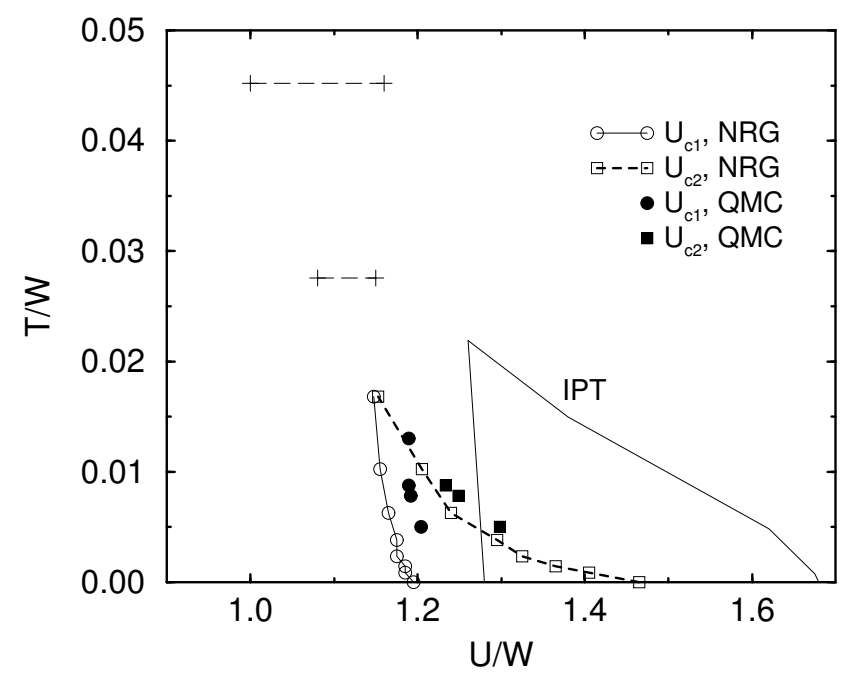

Figure 2.3: (taken from ref. [28]) The MH-MIT of the Hubbard model in infinite dimensions on the Bethe lattice as calculated by NRG [28], QMC [87], and iterated perturbation theory (IPT) [150]. The dashed lines for $T>T_{c}$ indicate the position and width of the crossover region. Note that the units refer to the bandwidth $W_{\text {Bethe }}$ of the Bethe lattice.

tion line is situated close to the upper critical value of the coexistence region [28]. Collecting all results, one can also deduce that the transition line is of first order and ends in two second-order critical end points at $T=0$ and $T=T_{\mathrm{c}}[150,48]$.

In order to locate the MH-MIT in the phase diagram fig. $2.2 \mathrm{~b}$, one should be aware of the fact that the DMFT calculations were performed using a different non-interacting band structure. Thus, energy units like the bandwidth cannot be compared directly. Following the discussion in section 7.2.2, the upper critical end point of the MH-MIT at $T_{\mathrm{c}} \approx 0.02 W_{\text {Bethe }}$ and $U_{\mathrm{c}} \approx 1.2 W_{\text {Bethe }}$ translates to $T_{\mathrm{c}} \approx$ $0.016 \mathrm{~W}=0.2 t$ and $U_{\mathrm{c}} \approx 0.98 \mathrm{~W}=11.7 t$, respectively, on the simple cubic lattice. This demonstrates in comparison to fig. $2.2 \mathrm{~b}$ that the MH-MIT is far below the Néel temperature $T_{\mathrm{N}} \approx 0.3 t$ for this particular interaction strength.

\subsubsection{Magnetic frustration}

The emergence of the MH-MIT from the antiferromagnetic phase as seen in fig. 2.1 is the most obvious qualitative difference between the phase diagram of $\mathrm{V}_{2} \mathrm{O}_{3}$ and that of the single-band Hubbard model. The predominance of antiferromagnetism in the Hubbard model can be attributed to its simplified lattice structure compared to the real material which exhibits a fairly complex corundum structure of vanadium ions surrounded by an octahedron of oxygen atoms (fig. 2.4a). The oxygen effectively mediates the hopping of electrons between vanadium ions. If we reduce the structure and show the vanadium ions only (fig $2.4 \mathrm{~b}$ ), the partly triangular structure of the 
a)

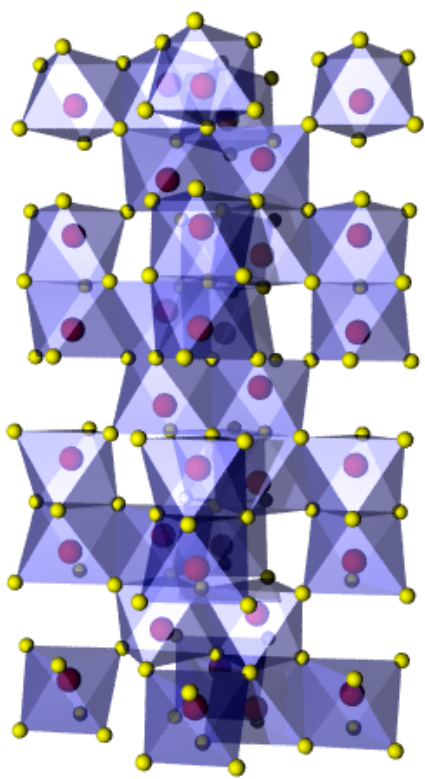

b)

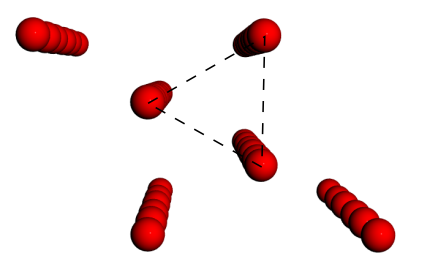

Figure 2.4: Panel a: the corundum structure of $\mathrm{V}_{2} \mathrm{O}_{3}$. Each vanadium atom (denoted by red spheres) is surrounded by a octahedron of oxygen atoms (yellow spheres). Panel b: The same structure reduced to the vanadium atoms and seen from above. The dashed lines highlight the triangular structure.

lattice becomes apparent. Antiferromagnetic order relies on a bipartite lattice, i. e., a lattice which can be divided into two sub-lattices. The spins point upwards on one sub-lattice and downwards on the other. A lattice with triangular structure elements is not bipartite. Hence it is impossible to establish a complete antiferromagnetic order where each spin is surrounded by spins of opposite direction only: the order is frustrated.

We can incorporate frustration into our model by introducing a hopping $t^{\prime}$ between next-nearest neighbors (denoted by $\langle\langle i, j\rangle\rangle$ ) to the Hamiltonian eq. (2.1) via

$$
H^{\prime}=H-t^{\prime} \sum_{\langle\langle i, j\rangle\rangle \sigma} c_{i \sigma}^{\dagger} c_{j \sigma}
$$

This will also affect the dispersion relation eq. (2.3) yielding

$$
\epsilon_{\boldsymbol{k}}=-2 t \sum_{i=1}^{3} \cos \left(k_{i}\right)-4 t^{\prime}\left[\cos \left(k_{1}\right) \cos \left(k_{2}\right)+\cos \left(k_{2}\right) \cos \left(k_{3}\right)+\cos \left(k_{1}\right) \cos \left(k_{3}\right)\right]
$$

with bandwidth

$$
W=\left\{\begin{array}{ll}
12 t & \text { for }\left|t^{\prime}\right| \leq t / 4 \\
8 t+16\left|t^{\prime}\right| & \text { for }\left|t^{\prime}\right|>t / 4
\end{array} .\right.
$$

DMFT calculations on frustrated Bethe lattices have shown that the antiferromagnetic phase of the Hubbard model becomes smaller with increasing $t^{\prime}$ up to the point where the MH-MIT emerges from the phase [137]. 


\subsection{Cold atoms in optical lattices}

Besides its applications in condensed matter physics, the Hubbard model plays an important role in the explanation of many-body phenomena observed in dilute, ultracold atomic gases [23]. Major advances in laser cooling [116] in the 1980s and 1990s led to the realization of artificial crystals of atoms bound by light at temperatures in the micro-Kelvin regime. These systems can present a remarkably clear realization of the Hubbard model and other quantum-mechanical lattice models [80]. The model parameters are often under precise control of the experimentalist and offer thus an opportunity to systematically study the properties of lattice models, hereby expressing the concept of a "quantum simulator".

Typically, neutral atoms - in most cases alkali metals - are confined in a magnetooptical trap [145], a device that applies a combination of magnetic trapping and Doppler cooling to reduce the temperature of the atoms. A system of laser beams with frequency $\omega$ slightly less than a resonant frequency $\omega_{\mathrm{R}}$ produces light which can only be absorbed when the beam is red-shifted due to the Doppler effect, i. e., when the atoms are moving towards the laser source. An additional varying magnetic quadrupole field causes a Zeeman shift towards the laser frequency $\omega$ which becomes stronger with increasing distance from the trap center, thus providing a further confinement mechanism.

After cooling, the atomic cloud is transfered to an optical dipole trap [52] consisting of several laser beams. When the atoms are placed in the light field, the oscillating electric field $\boldsymbol{E}(\boldsymbol{r}, t)$ induces an electric dipole moment $\boldsymbol{d}(\boldsymbol{r}, t)$ in an atom at coordinate $\boldsymbol{r}$. This leads to a Stark shift

$$
V(r)=-\langle\boldsymbol{d}(\boldsymbol{r}, t) \cdot \boldsymbol{E}(\boldsymbol{r}, t)\rangle=-\alpha(\omega)\left\langle\boldsymbol{E}^{2}(\boldsymbol{r}, t)\right\rangle
$$

of the atomic energy levels. Here $\alpha(\omega)$ denotes the atomic polarizability and $\langle\ldots\rangle$ a period average. The laser frequency $\omega=\omega_{\mathrm{R}}+\Delta$ is usually tuned far away from an atomic resonance frequency $\omega_{\mathrm{R}}$, such that spontaneous emission effects from resonant excitations can be neglected and the dynamic energy shift $V(r)$ behaves like a purely conservative potential. It is attractive for $\Delta<0$, when the induced dipole is in phase with the electric field $(\alpha(\omega)>0)$.

A stable optical trap can be realized by focusing the laser beam to a spot size $w$. If we assume a Gaussian intensity distribution along the radius $r$, the resulting potential has the form

$$
V_{\mathrm{T}}(r) \approx V_{0} \exp \left(\frac{-2 r^{2}}{w}\right),
$$

where the trap depth $V_{0}$ is proportional to the intensity of the beam. Expanding around $r=0$, the harmonic approximation of the potential radial to the propagation direction of the beam is given by

$$
V_{\mathrm{T}}(r) \approx \frac{V_{0}}{w^{2}} r^{2} .
$$


In order to confine the atoms in all spatial dimensions, two or more crossed laser can be used. If the trap depth is continuously lowered by decreasing the laser intensity, high-energy atoms leave the trap and the remaining ones thermalize at a lower temperature. Thus, an additional cooling effect is achieved by evaporative cooling [113].

A periodic potential can then be formed simply by overlapping two counterpropagating laser beams with identical wavelength $\lambda$. The interference between the two laser beams forms an optical standing wave creating a potential with a distance $\lambda / 2$ between two minima. By interfering more laser beams, one can obtain $1 \mathrm{D}, 2 \mathrm{D}$, and $3 \mathrm{D}$ periodic potentials. The potential as seen by the atoms is then

$$
V_{\mathrm{L}}(\boldsymbol{r})=\sum_{n=1}^{D} V_{0 n} \cos ^{2}\left(\frac{2 \pi r_{n}}{\lambda}\right)
$$

with the potential depths $V_{0 n}$ in each of the $D$ dimensions. This periodic potential can again be approximated around each minimum by a harmonic potential. An important advantage of using optical fields to create a periodic trapping potential is that the geometry and depth of the potential are under complete control.

Experiments on fermions are more difficult to realize than those on bosons. The main problem is the cooling process. In contrast to the bosonic case, two-body elastic scattering is mostly absent in a Fermi system at low temperature due to the Pauli exclusion principle. This inhibits thermalization and precludes direct implementation of evaporative cooling. To overcome this problem, one uses a mixture of bosons and fermions, which are cooled simultaneously [122]. Recent efforts aiming at the implementation of the Hubbard model in a cold gas experiment [95] has led to experimental signs of the Mott insulator [88, 163]. A major experimental achievement will be the detection of the antiferromagnetic phase, for which the slow and ill-understood equilibration rates, the limited number of detection methods, and cooling problems will have to be overcome.

\subsubsection{Theoretical modeling}

Early experiments on cold atoms focused on weakly interacting, dilute gases of bosons in optical or magnetic traps $[36,102]$ and were mostly conducted without a periodic potential. The main goal was to investigate quantum mechanical coherence which led, e.g., to the first experimental realization of a Bose-Einstein condensate [37]. More recently, the emphasis has shifted to strongly interacting systems. As first pointed out in ref. [79], a system of bosonic atoms in an optical lattice can be appropriately described by a Bose-Hubbard model. This identification can be generalized to a multitude of Hubbard type lattice models [80], including the fermionic Hubbard model. 
The Hubbard model can again be motivated by starting with the Hamiltonian for fermions of mass $m$ and spin $\sigma$ subject to the external potentials $V_{\mathrm{T}}(\boldsymbol{r})$ and $V_{\mathrm{L}}(\boldsymbol{r})$ of the previous section,

$$
\begin{aligned}
H= & \sum_{\sigma} \int \mathrm{d} \boldsymbol{r} \psi_{\sigma}^{\dagger}(\boldsymbol{r})\left(\frac{-\hbar^{2}}{2 m} \nabla^{2}+V_{\mathrm{L}}(\boldsymbol{r})+V_{\mathrm{T}}(\boldsymbol{r})\right) \psi_{\sigma}(\boldsymbol{r}) \\
& +\frac{1}{2} \frac{4 \pi a_{s} \hbar^{2}}{m} \int \mathrm{d} \boldsymbol{r} \psi_{\uparrow}^{\dagger}(\boldsymbol{r}) \psi_{\downarrow}^{\dagger}(\boldsymbol{r}) \psi_{\downarrow}(\boldsymbol{r}) \psi_{\uparrow}(\boldsymbol{r}) .
\end{aligned}
$$

Here the interatomic repulsion is already approximated by a local potential using $a_{s}$, the $s$-wave scattering length. Additionally, other energy bands are assumed to be high in energy and irrelevant for the effective description. Note that - in contrast to a solid - this condition can actually be controlled experimentally. An expansion in Wannier states at each lattice potential minimum yields analogous to the calculation in section 2.1

$$
H=-t \sum_{\langle i, j\rangle \sigma} c_{i \sigma}^{\dagger} c_{j \sigma}+\sum_{i \sigma} V_{i} n_{i \sigma}+U \sum_{i} n_{i \uparrow} n_{i \downarrow}
$$

with

$$
U=\frac{1}{2} \frac{4 \pi a_{s} \hbar^{2}}{m} \int \mathrm{d} \boldsymbol{r}|W(\boldsymbol{r})|^{4} .
$$

The notable difference to eq. (2.1) is the non-uniform onsite potential

$$
V_{i}=\int \mathrm{d} \boldsymbol{r} V_{\mathrm{T}}(\boldsymbol{r})\left|W\left(\boldsymbol{r}-\boldsymbol{r}_{i}\right)\right|^{2} \approx V_{\mathrm{T}}\left(\boldsymbol{r}_{i}\right)
$$

due to the trap. A closer inspection of the relevant energy scales shows that the approximations in the derivation are justified and that the coupling strengths can in principle be realized experimentally [80]. A sketch of an optical lattice system with the parameters of the Hubbard model is shown in fig. 2.5.

Various types of Hamiltonians of Bose and Fermi systems can thus be realized in optical lattices. This offers remarkably clean access to model Hamiltonians, that were originally developed in the context of condensed-matter physics, providing model systems for testing fundamental theoretical concepts. The observation of a quantum phase transition between a Mott insulator and a superfluid phase in a system of bosons [51] demonstrated the potential of this approach.

Theoretical modeling of atoms in a harmonic trap is more difficult than for homogeneous systems, because the trap breaks translational symmetry. This problem is most easily solved in one dimension. QMC studies on trapped 1D systems have been performed for both bosons [8] and fermions [147, 146]. For higher dimensional systems, especially for fermions on 3D lattices, one has to revert to approximation schemes.

One particularly common approximation $[163,38]$ is the local density approximation (LDA) which avoids the simulation of an spatially inhomogeneous system. 


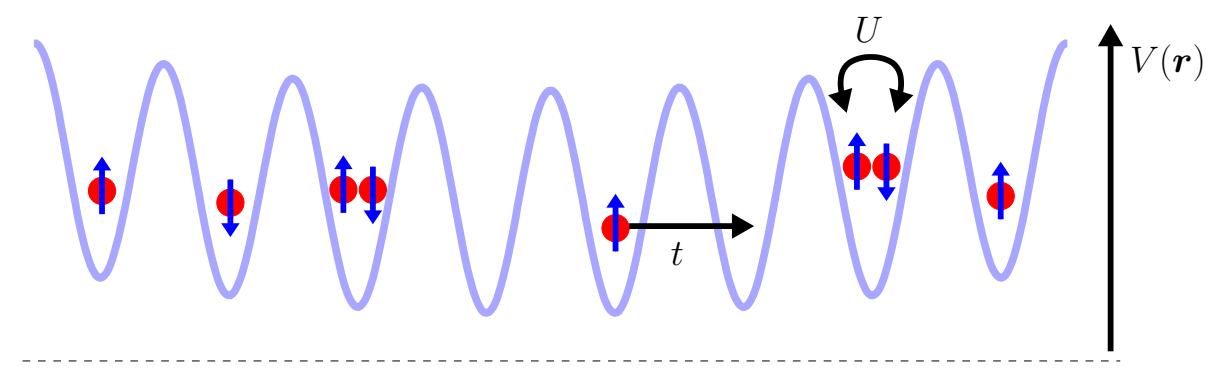

Figure 2.5: Sketch of an optical lattice system in one dimension. Atoms (depicted by red dots) with spin up/down (blue arrows) can move from one minimum of the lattice potential to the other. The system can be effectively described by a Hubbard model with local Coulomb repulsion $U$ and hopping $t$. The ratio between the parameters $t$ and $U$ can be tuned by changing the height of the trapping potential. Not the parabolic shape of the lattice potential.

Here one assumes that in the limit of large lattices with a slowly varying trapping potential the influence of the trap becomes local. Thus, a series of calculations of a homogeneous systems with chemical potentials $\mu-V_{\mathrm{T}}(\boldsymbol{r})$ is sufficient to take the trap geometry into account. There exist a few approximate schemes that do not rely on the LDA, e.g., the real-space DMFT [69, 171]. Calculations with these methods have shown that the LDA is indeed a very a good approximation, at least for simple thermodynamic observables [69, 161].

However, even within the LDA, the remaining task to solve the homogeneous Hubbard model on a 3D lattice with arbitrary filling is difficult. Since there are no exact methods available for this problem, most authors use approximate methods like DMFT [163, 38] or high temperature series expansions (HTSE) [161]. These calculations resulted in temperature and entropy estimates. The entropy is a particularly interesting quantity, because the atoms are well isolated from their environment and experiments are therefore carried out adiabatically at constant entropy. Since a direct experimental temperature measurement in the dilute atomic gas is not possible, thermometry is also an important issue. The temperature can be inferred from comparisons of experimental observables with those from simulations of the Hubbard model. Previous works focused on the double occupancy as a experimentally measurable candidate for thermometry [89, 49]. Results from these calculations have already been applied to experimental data [89].

We provide entropy estimates and observables for thermometry based on an approximate solution of the homogeneous 3D Hubbard model with the DCA simulations (see chapter 5). We focus on the direct vicinity of the Néel phase providing data that could be experimentally valuable for the detection of the antiferromagnetic phase. 


\section{Dynamical cluster approximation}

The dynamical cluster approximation (DCA) is an approximation scheme, that maps the infinite lattice Hamiltonian described in the previous chapter on a finite cluster embedded in a mean-field. Details and properties of this mapping are the subject of the present chapter. Means to solve the resulting effective cluster problem are discussed in chapter 4 .

The numerical treatment of lattice models in the thermodynamic limit is generally impossible. Limited computational resources restrict the tractable system sizes to finite clusters with only a small number of lattice sites. Numerically exact solutions of finite lattice systems are usually gained by exact diagonalization [192] or QMC methods. Exact diagonalization is severely limited by the exponential growth of the computational effort with system size, whereas QMC suffers from the sign problem [184], that also restricts the simulations to small systems and special cases, such as particle-hole symmetric situations.

Thus, a finite-size extrapolation to the thermodynamic limit is very difficult. In particular, the identification of ordered phases, which requires a reliable finite-size scaling, becomes exceedingly complicated. A direct investigation of the properties of ordered phases possibly present in the thermodynamic limit is not possible, because finite systems cannot exhibit a spontaneously broken symmetry. Therefore, a suitable approximation scheme allowing calculations directly in the thermodynamic limit while including dynamical correlations in a controlled way is highly desirable. Such methods have been devised under the name of dynamical mean-field theory (DMFT) $[48,96]$ and cluster mean-field theories [109].

The DMFT maps the lattice problem onto an effective single-site model embedded in a mean-field. The single-site problem is solved by a suitable impurity solver. Examples for impurity solvers are renormalization techniques [27], exact diagonalization [30] and, again, QMC algorithms, which will be discussed in detail in chapter 4. An important technical aspect in the context of QMC algorithms is that the DMFT completely avoids or at least strongly limits the sign problem in many cases. The mean-field must be determined self-consistently, similar to well known mean-field theories such as the Weiss theory for spin models [191]. Although it differs from these theories in this respect, that it is dynamic, i. e., time dependent, and can thus treat quantum fluctuations.

The DMFT becomes exact in the limit of infinite coordination number or equivalently infinite dimensions $[118,126]$. However, non-local corrections become important in finite dimensions. Non-local many-body correlations are necessary to study 
the interplay or competition of formation of a Fermi liquid and more exotic states of matter. Thus, the DMFT is unable to capture the physics of, e.g., localization in disordered systems, spin waves in quantum-mechanical spin models, or spin-liquid physics in correlated electronic systems.

Cluster mean-field theories approximate an infinite lattice problem by a finite cluster embedded in a mean field, similar to the spirit adopted in DMFT. Within this approximation, all physical effects local to the cluster including quantum fluctuations and short ranged correlations are treated exactly. In contrast to conventional finite size calculations, long range correlation are captured as well, but in a meanfield treatment only. In the limit of infinite cluster size the original lattice problem is regained. If the cluster consists of only one lattice site, the approximation simplifies to DMFT, which only captures fluctuations local to the single lattice site and neglects all spatial correlations. Thus, cluster mean-field theories can be understood as a systematic extension to the DMFT by incorporating more and more short-ranged correlations as the cluster size increases.

Cluster mean-field theories have been applied to different quantum-mechanical lattice models. Examples include the Falicov-Kimbal model [71, 70], the 1D Hubbard model [24], the 2D Hubbard model with [98, 135] and without [77, 125, 55] frustration, and the 3D Hubbard model [92].

Two distinct methods have been developed in recent years, the DCA $[71,70]$ and the cellular dynamical mean-field theory (CDMFT) [97]. The difference between the two methods is mostly of a technical nature. While the CDMFT violates translational symmetry, the DCA restores this property of the infinite lattice and can therefore be efficiently formulated in momentum space. This simplifies, e.g., the calculation of two-particle properties [70, 84]. The two methods also differ in their finite-size scaling behavior [110]. We used the DCA for this work exclusively and will therefore only briefly mention details of the CDMFT algorithm in the course of this chapter.

\subsection{Cluster structure}

We study the simple cubic lattice in three dimensions. Each site of the lattice is described by a vector

$$
\boldsymbol{r}=n_{1} \hat{\boldsymbol{x}}+n_{2} \hat{\boldsymbol{y}}+n_{3} \hat{\boldsymbol{z}},
$$

where $n_{1}, n_{2}, n_{3}$ are integers and $\hat{\boldsymbol{x}}, \hat{\boldsymbol{y}}, \hat{\boldsymbol{z}}$ orthonormal vectors in the three spatial dimensions. We tile the infinite lattice by finite-size clusters with periodic boundary conditions by defining a parallelepiped spanned by the $3 \times 3$ matrix

$$
\mathbf{C}:=\left(\boldsymbol{l}_{1}, \boldsymbol{l}_{2}, \boldsymbol{l}_{3}\right):=\left(\begin{array}{lll}
l_{11} & l_{12} & l_{13} \\
l_{21} & l_{22} & l_{23} \\
l_{31} & l_{32} & l_{33}
\end{array}\right)
$$



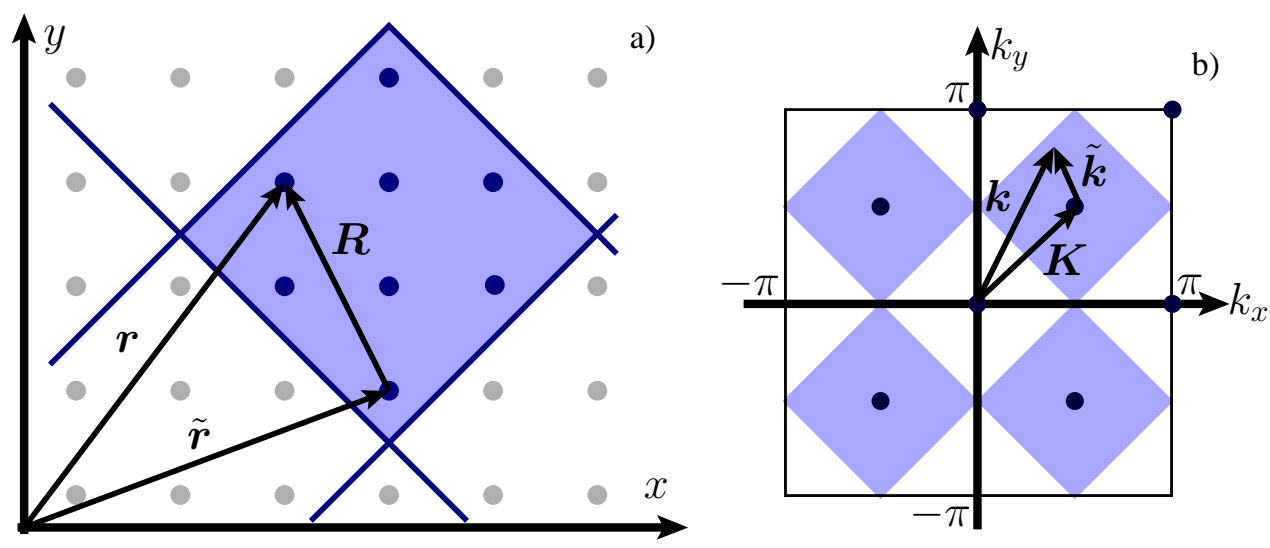

Figure 3.1: The DCA illustrated for a 8-site cluster in two dimensions. In real space (a) the origin of a cluster is labelled by $\tilde{\boldsymbol{r}}$. Each site of the cluster is identified by $\boldsymbol{R}$. Fourier transformation maps the coordinate $\boldsymbol{r}=\tilde{\boldsymbol{r}}+\boldsymbol{R}$ of each lattice site to a vector $\boldsymbol{k}$ in the first Brillouin zone (b). The cluster momentum $\boldsymbol{K}$ now identifies the centre of a cell in momentum space. All points inside this cell are accessible by $\tilde{\boldsymbol{k}}$. The DCA integrates out $\tilde{\boldsymbol{k}}$ and thus replaces the full $\boldsymbol{k}$-dependence of the lattice by the cells labelled by $\boldsymbol{K}$.

where the entries $l_{i j}$ are again integers. The infinite lattice can be reconstructed by translating the origin of the cluster by

$$
\tilde{\boldsymbol{r}}=n_{1} \boldsymbol{l}_{1}+n_{2} \boldsymbol{l}_{2}+n_{3} \boldsymbol{l}_{3}
$$

and thus tiling the whole lattice with the parallelepiped. The sites inside each cluster are described by the vector $\boldsymbol{R}$, so that $\boldsymbol{r}=\tilde{\boldsymbol{r}}+\boldsymbol{R}$. The size $N_{\mathrm{c}}$ of the cluster is equal to the volume of the parallelepiped, $N_{\mathrm{c}}=\operatorname{det} \mathbf{C}$. An example for such a cluster in two dimensions is given in fig. 3.1a. Although 3D lattices are the main focus of this work, we will restrict the examples in this chapter to the square lattice in $2 \mathrm{D}$ for simplicity. In case of $2 \mathrm{D}$ lattices $\mathbf{C}$ reduces to a $2 \times 2$-matrix. The figure shows the cluster defined by

$$
C=\left(\begin{array}{cc}
2 & 2 \\
2 & -2
\end{array}\right)
$$

Because the DCA is formulated in momentum space, we also need to Fouriertransform the cluster structure. In reciprocal space, the vector $\boldsymbol{r}$ is mapped onto an element $\boldsymbol{k}$ of the first Brillouin zone. The vectors $\boldsymbol{R}$ and $\tilde{\boldsymbol{r}}$ are transformed into $\boldsymbol{K}$ and $\tilde{\boldsymbol{k}}$, respectively. The cluster structure described above is now equivalent to tiling the first Brillouin zone into $N_{\mathrm{c}}$ non-overlapping cells, each represented by its central momentum $\boldsymbol{K}$. All vectors inside the volume $V$ of the cell are accessible by $\tilde{\boldsymbol{k}}$ (cf. fig. $3.1 \mathrm{~b}$ for an example). This coarse-graining of the first Brillouin zone forms the basis of the DCA, the details of which are given in the next section. 


\subsection{Outline of the method}

The central quantity for the DCA is the single particle Green function in imaginary time $\tau$, defined by

$$
G_{\sigma i j}(\tau)=-\left\langle T_{\tau} c_{i \sigma}(\tau) c_{j \sigma}^{\dagger}\right\rangle
$$

Here $T_{\tau}$ is the imaginary-time ordering operator, $\langle\ldots\rangle$ denotes a thermal expectation value and $c_{\sigma i}(\tau)=e^{-H \tau} c_{\sigma i} e^{H \tau}$. The DCA is also applicable to Green functions depending on real times. However, we will restrict ourselves to imaginary times and frequencies in the course of the chapter having in mind the application of imaginarytime-based QMC methods. The spatial and temporal Fourier transform of the Green function eq. (3.5) is

$$
G_{\sigma \boldsymbol{k}}\left(\mathrm{i} \omega_{n}\right)=\frac{1}{N_{\mathrm{c}}} \sum_{i j} \exp \left[\mathrm{i} \boldsymbol{k} \cdot\left(\boldsymbol{r}_{i}-\boldsymbol{r}_{j}\right)\right] \int_{0}^{\beta} \mathrm{d} \tau \mathrm{e}^{\mathrm{i} \omega_{n} \tau} G_{\sigma i j}(\tau),
$$

where $\omega_{n}=(2 n+1) \pi / \beta$ with $n \in \mathbb{Z}$ and $\beta=1 / k_{\mathrm{B}} T$ are the fermionic Matsubara frequencies. As usual, $T$ denotes the temperature and $k_{\mathrm{B}}$ Boltzmann's constant. The self-energy $\Sigma_{\sigma \boldsymbol{k}}\left(\mathrm{i} \omega_{n}\right)$ is defined via Dyson's equation

$$
\Sigma_{\sigma \boldsymbol{k}}\left(\mathrm{i} \omega_{n}\right)=g_{\sigma \boldsymbol{k}}\left(\mathrm{i} \omega_{n}\right)^{-1}-G_{\sigma \boldsymbol{k}}\left(\mathrm{i} \omega_{n}\right)^{-1}
$$

using the bare Green function

$$
g_{\sigma \boldsymbol{k}}\left(\mathrm{i} \omega_{n}\right)=\frac{1}{\mathrm{i} \omega_{n}+\mu-\epsilon_{\boldsymbol{k}}},
$$

where $\mu$ denotes the chemical potential and $\epsilon_{\boldsymbol{k}}$ the bare dispersion eq. (2.3). The bare Green function is the Green function of the non-interacting system.

The full $\boldsymbol{k}$-dependence of the self-energy is now approximated by the self-energies at the discrete set of $N_{\mathrm{c}}$ cluster momenta $\boldsymbol{K}$ by replacing $\Sigma_{\sigma \boldsymbol{k}}\left(\mathrm{i} \omega_{n}\right)$ by $\Sigma_{\sigma \boldsymbol{K}}\left(\mathrm{i} \omega_{n}\right)$. Here $\Sigma_{\sigma \boldsymbol{K}}\left(\mathrm{i} \omega_{n}\right)$ is taken to be constant in each cluster cell volume $V$. This coarsegraining of the self-energy is the central approximation of the DCA. It will be further motivated in section 3.3. In the limit $N_{\mathrm{c}}=1$, only the single cluster momentum $\boldsymbol{K}=0$ is kept and the coarse graining is extended to the whole first Brillouin zone. This purely local limit is identical to the DMFT. If we apply this approximation to the Green function eq. (3.6)

$$
G_{k}=\frac{1}{g_{k}^{-1}-\Sigma_{k}}
$$

(omitting the dependency on $\mathrm{i} \omega_{n}$ and $\sigma$ from now on) and average over the volume $V$ of the cell corresponding to cluster momentum $\boldsymbol{K}$, we obtain the coarse-grained Green function

$$
\bar{G}_{\boldsymbol{K}}=\frac{1}{V} \int \mathrm{d} \tilde{\boldsymbol{k}} \frac{1}{g_{\boldsymbol{K}+\tilde{\boldsymbol{k}}}^{-1}-\Sigma_{\boldsymbol{K}}},
$$




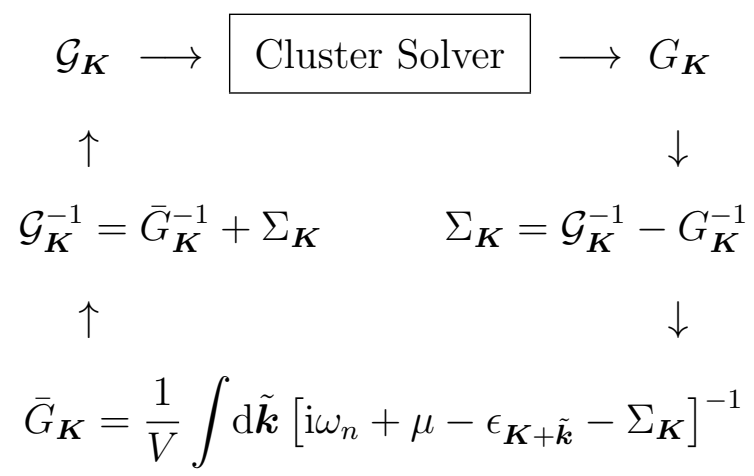

Figure 3.2: The self-energy $\Sigma_{\sigma \boldsymbol{K}}\left(\mathrm{i} \omega_{n}\right)$ is determined self-consistently by iterating the depicted procedure until convergence is reached. The bottom line shows the calculation of the coarse-grained Green function $\bar{G}_{\boldsymbol{K}}$ by averaging over the momentum cell centered around $\boldsymbol{K}$ via integrating $\tilde{\boldsymbol{k}}$ over the volume $V$ of the cell. The dependency of the Green functions and the self-energy on $i \omega_{n}$ and $\sigma$ is omitted for simplicity.

where only the momentum dependence of the self-energy is approximated, while the bare Green function still depends on $\boldsymbol{k}=\boldsymbol{K}+\tilde{\boldsymbol{k}}$. Finally, an effective non-interacting cluster Green function is defined via

$$
\mathcal{G}_{\boldsymbol{K}}^{-1}=\bar{G}_{\boldsymbol{K}}^{-1}+\Sigma_{\boldsymbol{K}}
$$

With this set of quantities and using a suitable method to solve the effective cluster defined by $\mathcal{G}_{\boldsymbol{K}}$ and the interaction $U$, one can determine the self-energy $\Sigma_{\boldsymbol{K}}$ selfconsistently.

Fig. 3.2 depicts outlines of the iterative procedure to determine the cluster selfenergy $\Sigma_{\boldsymbol{K}}$. The iteration starts with an initial guess for the self-energy. One usually takes the free solution $\Sigma_{\boldsymbol{K}}=0$ and calculates $\bar{G}_{\boldsymbol{K}}=\mathcal{G}_{\boldsymbol{K}}$. This quantity serves as input to the cluster solver, which treats $\mathcal{G}_{K}$ as the effective bare Green function of the cluster and calculates the interacting Green function $G_{\boldsymbol{K}}$ subject to the interaction $U$. This task is performed by the QMC methods detailed in chapter 4 and is by far the most time consuming part of the self-consistency loop. The solution defines a new self-energy via Dyson's equation,

$$
\Sigma_{K}=\mathcal{G}_{K}^{-1}-G_{K}^{-1}
$$

A recalculation of $\bar{G}_{\boldsymbol{K}}$ and $\mathcal{G}_{\boldsymbol{K}}$ follows using the new self-energy. Subsequently, the interacting Green function $G_{\boldsymbol{K}}$ is again calculated by the cluster solver. Additional iterations follow until convergence of the self-energy is accomplished. 


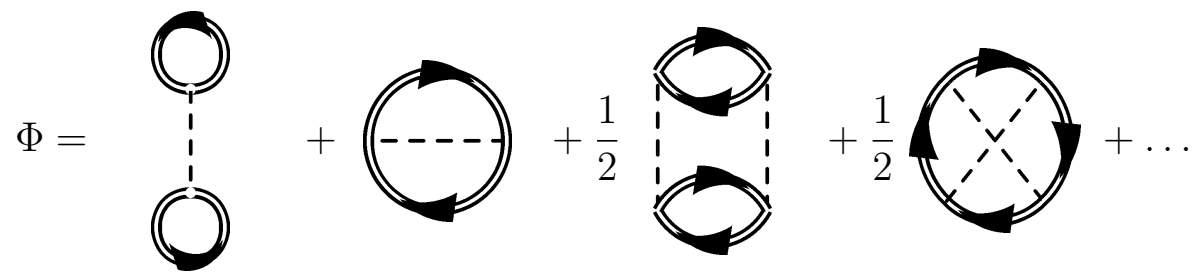

Figure 3.3: First and second order contributions to the generating functional $\Phi$. Dashed lines represent the interaction $U$. Double line arrows represent the Green function $G$. Each diagram of order $n$ is weighted by a factor $\frac{1}{n}$.

\subsection{Diagrammatic motivation}

In order to motivate the central approximation of the last section, i. e., the coarsegraining of the self-energy, we provide a diagrammatic argument of the DCA equations.

A thermodynamically consistent approximation may be constructed by requiring that the self-energy fulfill $[10,11]$

$$
g^{-1}-G^{-1}=\Sigma=\frac{\delta \Phi[G, U]}{\delta G},
$$

where $\Phi[G, U]$ denotes the Luttinger-Ward generating functional defined as a skeletal sum over all compact, closed, connected graphs constructed from $G$ and the interaction $U$ [106]. A few lowest order contributions of $\Phi$ can be seen in fig 3.3. On the one hand, eq. (3.13) demands that the self-energy be a functional derivative of $\Phi$ with respect to the Green function $G$. Thus, any approximation scheme should be performed on the generating functional $\Phi$. On the other hand, the potentially approximated self-energy needs to fulfill Dyson's equation thereby creating a self-consistency condition.

The relation between $\Phi$ and the grand-canonical potential $\Omega$ is $[106,1]$

$$
\Omega[G, U]=-\frac{1}{\beta}(\Phi[G, U]-\operatorname{Tr} \ln (-G)-\operatorname{Tr} \Sigma G)
$$

where the trace indicates summation over lattice momenta, Matsubara frequencies, and spin. Subject to the condition eq. (3.13), $\Omega$ is stationary with respect to $G$, i. e.,

$$
\frac{\delta \Omega[G, U]}{\delta G}=-\frac{1}{\beta}\left(\frac{\delta \Phi[G, U]}{\delta G}-\Sigma\right) \stackrel{(3.13)}{=} 0
$$

The potential $\Omega$ will still be stationary, if one uses an approximated generating functional $\Phi_{\mathrm{c}}$ and consequently an approximated self-energy $\Sigma_{\mathrm{c}}$ by enforcing eq. (3.13), i. e., the self-consistency equation $\Sigma_{\mathrm{c}}=g^{-1}-G^{-1}$. Such an approximation is thermodynamically consistent: Observables calculated from $G$ agree with those calculated 
from $\Omega$. It conserves quantities derived from the potential such as particle number and pressure. That means that the approximation also preserves conservation laws [10] and is therefore called a conserving approximation.

The DCA does not restrict the number of diagrams in $\Phi$ as is common, for example, in the Hartree-Fock approximation, which takes only the first order diagrams in fig. 3.3 into account. The approximation is introduced into the momentum conservation at internal vertices of each diagram which is described by the Laue function

$$
\Delta=\sum_{\boldsymbol{r}} \mathrm{e}^{\mathrm{i} \boldsymbol{r} \cdot\left(\boldsymbol{k}_{1}+\boldsymbol{k}_{2}+\cdots-\boldsymbol{k}_{1}^{\prime}-\boldsymbol{k}_{2}^{\prime}-\ldots\right)} \propto \delta_{\boldsymbol{k}_{1}+\boldsymbol{k}_{2}+\ldots, \boldsymbol{k}_{1}^{\prime}+\boldsymbol{k}_{2}^{\prime}+\ldots} .
$$

Here, $\boldsymbol{k}_{1}, \boldsymbol{k}_{2}, \ldots$ are the momenta entering the vertex and $\boldsymbol{k}_{1}^{\prime}, \boldsymbol{k}_{2}^{\prime}, \ldots$ the momenta leaving it. If one introduces the cluster structure and writes $\boldsymbol{k}=\boldsymbol{K}+\tilde{\boldsymbol{k}}$ and $\boldsymbol{r}=\boldsymbol{R}+\tilde{\boldsymbol{r}}$, several approximation schemes are possible. If all phase factors including $\tilde{\boldsymbol{r}}$ are omitted, the result is the CDMFT [97]. In this approximation, the factors including $\tilde{\boldsymbol{k}}$ violate the translational symmetry of the clusters. The CDMFT is therefore formulated in real space. In order to regain translational symmetry, the phase factors including $\tilde{\boldsymbol{k}}$ are neglected as well resulting in the Laue function of the DCA,

$$
\Delta_{\mathrm{DCA}}=\sum_{\boldsymbol{R}} \mathrm{e}^{\mathrm{i} \boldsymbol{R} \cdot\left(\boldsymbol{K}_{1}+\boldsymbol{K}_{2}+\cdots-\boldsymbol{K}_{1}^{\prime}-\boldsymbol{K}_{2}^{\prime}-\ldots\right)} \propto \delta_{\boldsymbol{K}_{1}+\boldsymbol{K}_{2}+\ldots, \boldsymbol{K}_{1}^{\prime}+\boldsymbol{K}_{2}^{\prime}+\ldots} .
$$

If the conservation of momentum is ignored completely, i. e., $\Delta=1$, we regain the DMFT [126]. The integral over $\tilde{\boldsymbol{k}}$ is no longer restricted to any momentum conservation law if we use $\Delta_{\mathrm{DCA}}$ in each diagram of $\Phi[G, U]$ and can therefore be performed freely. Thus, each Green function $G$ and interaction $U$ is replaced by it's coarse-grained counterparts,

$$
\bar{G}_{\boldsymbol{K}}=\frac{1}{V} \int \mathrm{d} \tilde{\boldsymbol{k}} G_{\boldsymbol{K}+\tilde{\boldsymbol{k}}} \quad \text { and } \quad \bar{U}_{\boldsymbol{K}}=\frac{1}{V} \int \mathrm{d} \tilde{\boldsymbol{k}} U_{\boldsymbol{K}+\tilde{\boldsymbol{k}}} .
$$

The coarse-graining of the interaction is trivial in the case of the simple Hubbard model with only local interactions. The result of this procedure is an approximated $\Phi\left[\bar{G}_{\boldsymbol{K}}, \bar{U}_{\boldsymbol{K}}\right]$ which generates the cluster self-energy

$$
\Sigma_{\boldsymbol{K}}=\frac{\delta \Phi\left[\bar{G}_{\boldsymbol{K}}, \bar{U}_{\boldsymbol{K}}\right]}{\delta \bar{G}_{\boldsymbol{K}}} .
$$

By imposing eq. (3.13) it becomes apparent that the self-energy of the whole lattice is replaced by the cluster self-energy which depends on cluster momenta $\boldsymbol{K}$ only,

$$
g_{k}^{-1}-G_{k}=\Sigma_{k}=\Sigma_{K} .
$$

Integrating over $\tilde{\boldsymbol{k}}$, one derives

$$
\bar{G}_{\boldsymbol{K}}=\frac{1}{V} \int \mathrm{d} \tilde{\boldsymbol{k}} \frac{1}{g_{\boldsymbol{K}+\tilde{\boldsymbol{k}}}^{-1}-\Sigma_{\boldsymbol{K}}},
$$

which was postulated previously in eq. (3.10). 


\subsection{Effective cluster model}

The self-consistency scheme depicted in section 3.2 requires a tool to solve the cluster problem described by the effective bare Green function $\mathcal{G}_{\boldsymbol{K}}$ eq. (3.11) and the local Coulomb repulsion $U$. In the path integral formulation the partition function of this system is [129]

$$
Z=\int \mathcal{D}\left[\phi^{*}, \phi\right] \mathrm{e}^{-S\left[\phi^{*}, \phi\right]}
$$

The measure

$$
\mathcal{D}\left[\phi^{*}, \phi\right]=\prod_{\boldsymbol{K} \sigma} \mathcal{D} \phi_{\boldsymbol{K} \sigma}^{*} \mathcal{D} \phi_{\boldsymbol{K} \sigma}=\lim _{M \rightarrow \infty} \prod_{j=1}^{M} \prod_{\boldsymbol{K} \sigma} \mathrm{d} \phi_{\boldsymbol{K} \sigma}^{*}\left(\tau_{j}\right) \mathrm{d} \phi_{\boldsymbol{K} \sigma}\left(\tau_{j}^{\prime}\right)
$$

denotes a path integral over the Grassmann variables $\phi_{\boldsymbol{K} \sigma}(\tau)$. The action $S\left[\phi^{*}, \phi\right]=$ $S_{0}\left[\phi^{*}, \phi\right]+S_{U}\left[\phi^{*}, \phi\right]$ can be divided into two parts. The non-interacting part

$$
\begin{aligned}
S_{0}\left[\phi^{*}, \phi\right] & =-\int_{0}^{\beta} \mathrm{d} \tau \int_{0}^{\beta} \mathrm{d} \tau^{\prime} \sum_{\boldsymbol{K} \sigma} \phi_{\boldsymbol{K} \sigma}^{*}(\tau) \mathcal{G}_{\sigma \boldsymbol{K}}^{-1}\left(\tau-\tau^{\prime}\right) \phi_{\boldsymbol{K} \sigma}\left(\tau^{\prime}\right) \\
& =-\frac{1}{\beta} \sum_{\mathrm{i} \omega_{n}} \sum_{\boldsymbol{K} \sigma} \phi_{\boldsymbol{K} \sigma}^{*}\left(\mathrm{i} \omega_{n}\right) \mathcal{G}_{\sigma \boldsymbol{K}}^{-1}\left(\mathrm{i} \omega_{n}\right) \phi_{\boldsymbol{K} \sigma}\left(\mathrm{i} \omega_{n}\right)
\end{aligned}
$$

depends on $\mathcal{G}_{\boldsymbol{K} \sigma}\left(\mathrm{i} \omega_{n}\right)$ only. The interaction part

$$
S_{U}\left[\phi^{*}, \phi\right]=U \int_{0}^{\beta} \mathrm{d} \tau \sum_{i} \phi_{i \uparrow}^{*}(\tau) \phi_{i \uparrow}(\tau) \phi_{i \downarrow}^{*}(\tau) \phi_{i \downarrow}(\tau),
$$

is most easily written in real space using the local Coulomb repulsion $U$ and the real space equivalent $\phi_{i \sigma}(\tau)$ of the Grassmann variables.

It is also possible to formulate the cluster model using a specific Hamiltonian description. This formulation directly reflects the image of a cluster embedded in a mean field. Expressing the bare dispersion

$$
\epsilon_{\boldsymbol{K}+\tilde{\boldsymbol{k}}}=\bar{\epsilon}_{\boldsymbol{K}}+\delta \epsilon_{\boldsymbol{K}}(\tilde{\boldsymbol{k}})
$$

via the averaged bare dispersion $\bar{\epsilon}_{\boldsymbol{K}}=\frac{1}{V} \int \mathrm{d} \tilde{\boldsymbol{k}} \epsilon_{\boldsymbol{K}+\tilde{\boldsymbol{k}}}$ and the deviation $\delta \epsilon_{\boldsymbol{K}}(\tilde{\boldsymbol{k}})$ we rewrite the coarse-grained Green function eq. (3.10),

$$
\bar{G}_{\boldsymbol{K}}\left(\mathrm{i} \omega_{n}\right)^{-1}=\mathrm{i} \omega_{n}-\bar{\epsilon}_{\boldsymbol{K}}+\mu-\Sigma_{\boldsymbol{K}}\left(\mathrm{i} \omega_{n}\right)-\Gamma_{\boldsymbol{K}}\left(\mathrm{i} \omega_{n}\right),
$$

or alternatively

$$
\mathcal{G}_{\boldsymbol{K}}\left(\mathrm{i} \omega_{n}\right)^{-1}=\mathrm{i} \omega_{n}-\bar{\epsilon}_{\boldsymbol{K}}+\mu-\Gamma_{\boldsymbol{K}}\left(\mathrm{i} \omega_{n}\right)
$$


which defines the hybridization function

$$
\Gamma_{\boldsymbol{K}}\left(\mathrm{i} \omega_{n}\right)=\frac{\frac{1}{V} \int \mathrm{d} \tilde{\boldsymbol{k}} \delta \epsilon_{\boldsymbol{K}}^{2}(\tilde{\boldsymbol{k}}) G_{\boldsymbol{K}+\tilde{\boldsymbol{k}}}\left(\mathrm{i} \omega_{n}\right)}{1+\frac{1}{V} \int \mathrm{d} \tilde{\boldsymbol{k}} \delta \epsilon_{\boldsymbol{K}}(\tilde{\boldsymbol{k}}) G_{\boldsymbol{K}+\tilde{\boldsymbol{k}}}\left(\mathrm{i} \omega_{n}\right)} .
$$

In this formulation $\bar{G}_{\boldsymbol{K}}\left(\mathrm{i} \omega_{n}\right)$ already reflects the structure of the effective cluster model: The bare cluster is described by the bare dispersion $\bar{\epsilon}_{\boldsymbol{K}}$. The interaction due to the local repulsion $U$ is captured by $\Sigma_{\boldsymbol{K}}\left(\mathrm{i} \omega_{n}\right)$, whereas $\Gamma_{\boldsymbol{K}}\left(\mathrm{i} \omega_{n}\right)$ represents the dynamic coupling of the cluster to the surrounding bath. Let us now consider the effective cluster Hamiltonian

$$
\begin{aligned}
H_{\mathrm{c}} & =\sum_{\boldsymbol{K} \sigma}\left(\bar{\epsilon}_{\boldsymbol{K}}-\mu\right) c_{\boldsymbol{K} \sigma}^{\dagger} c_{\boldsymbol{K} \sigma}+U \sum_{i \sigma} n_{i \uparrow} n_{i \downarrow} \\
& +\sum_{\boldsymbol{K} \sigma} \int \mathrm{d} \tilde{\boldsymbol{k}} \zeta_{\boldsymbol{K}+\tilde{\boldsymbol{k}}} a_{\boldsymbol{K}+\tilde{\boldsymbol{k}} \sigma}^{\dagger} a_{\boldsymbol{K}+\tilde{\boldsymbol{k}} \sigma} \\
& +\sum_{\boldsymbol{K} \sigma} \int \mathrm{d} \tilde{\boldsymbol{k}}\left(V_{\boldsymbol{K}}(\tilde{\boldsymbol{k}}) c_{\boldsymbol{K} \sigma}^{\dagger} a_{\boldsymbol{K}+\tilde{\boldsymbol{k}} \sigma}+\text { H. c. }\right) .
\end{aligned}
$$

The first line is the finite cluster with the usual Hubbard interaction. The second line introduces a non-interacting infinite bath represented by auxiliary fermionic operators $a_{\boldsymbol{k} \sigma}^{(\dagger)}$ and an unknown energy dispersion $\zeta_{\boldsymbol{k}}$. The bath is coupled to the cluster via coupling amplitudes $V_{\boldsymbol{K}}(\tilde{\boldsymbol{k}})$ (unknown as well). The action of this system is

$$
\begin{aligned}
S_{\mathrm{c}}\left[\phi^{*}, \phi, \psi^{*}, \psi\right]= & \int_{0}^{\beta} \mathrm{d} \tau \sum_{\boldsymbol{K} \sigma} \phi_{\boldsymbol{K} \sigma}^{*}(\tau)\left(\frac{\partial}{\partial \tau}+\bar{\epsilon}_{\boldsymbol{K}}-\mu\right) \phi_{\boldsymbol{K} \sigma}(\tau)+S_{U}\left[\phi^{*}, \phi\right] \\
& +\int_{0}^{\beta} \mathrm{d} \tau \sum_{\boldsymbol{K} \sigma} \int \mathrm{d} \tilde{\boldsymbol{k}} \psi_{\boldsymbol{K}+\tilde{\boldsymbol{k}} \sigma}^{*}(\tau)\left(\frac{\partial}{\partial \tau}+\zeta_{\boldsymbol{K}+\tilde{\boldsymbol{k}}}\right) \psi_{\boldsymbol{K}+\tilde{\boldsymbol{k}} \sigma}(\tau) \\
& +\int_{0}^{\beta} \mathrm{d} \tau \sum_{\boldsymbol{K} \sigma} \int \mathrm{d} \tilde{\boldsymbol{k}}\left(\phi_{\boldsymbol{K} \sigma}^{*}(\tau) V_{\boldsymbol{K}}(\tilde{\boldsymbol{k}}) \psi_{\boldsymbol{K}+\tilde{\boldsymbol{k}} \sigma}(\tau)+\text { H.c. }\right),
\end{aligned}
$$

where $\phi_{\boldsymbol{K} \sigma}^{(*)}$ and $\psi_{\boldsymbol{k} \sigma}^{(*)}$ are the Grassmann variables to the corresponding operators $c_{\boldsymbol{K} \sigma}^{(\dagger)}$ and $a_{\boldsymbol{k} \sigma}^{(\dagger)}$, respectively. The bath variables $\psi_{\boldsymbol{k} \sigma}^{(*)}$ only appear in a biquadratic form and can be integrated out yielding the bath contribution $Z_{\text {bath }}$ to the partition function. Using the Gaussian integration formula for Grassmann variables

$$
\int \prod_{i} \mathrm{~d} \xi_{i}^{*} \mathrm{~d} \xi_{i} \exp \left(-\sum_{i j} \xi_{i}^{*} A_{i j} \xi_{j}+\sum_{i}\left[J^{*} \xi_{i}+\xi^{*} J\right]\right)=\operatorname{det} A \exp \left(J^{*} A^{-1} J\right)
$$


and identifying $\xi_{i}=\psi_{\boldsymbol{k} \sigma}(\tau), A=\delta_{\boldsymbol{k} \boldsymbol{k}^{\prime}} \delta_{\sigma \sigma^{\prime}} \delta\left(\tau-\tau^{\prime}\right)\left(\frac{\partial}{\partial \tau}-\zeta_{\boldsymbol{k}}\right)$, and $J=V_{\boldsymbol{K}}(\tilde{\boldsymbol{k}}) \psi_{\boldsymbol{k} \sigma}(\tau)$, the partition function of the effective model becomes

$$
\begin{aligned}
Z_{\mathrm{c}} & =\int \mathcal{D}\left[\phi^{*}, \phi\right] \mathcal{D}\left[\psi^{*}, \psi\right] \mathrm{e}^{-S_{\mathrm{c}}\left[\phi^{*}, \phi, \psi^{*}, \psi\right]} \\
& =Z_{\text {bath }} \int \mathcal{D}\left[\phi^{*}, \phi\right] \mathrm{e}^{-S_{0}\left[\phi^{*}, \phi\right]-S_{U}\left[\phi^{*}, \phi\right]}
\end{aligned}
$$

with

$$
\begin{aligned}
S_{0}\left[\phi^{*}, \phi\right]= & \int_{0}^{\beta} \mathrm{d} \tau \sum_{\boldsymbol{K} \sigma} \phi_{\boldsymbol{K} \sigma}^{*}(\tau)\left(\frac{\partial}{\partial \tau}+\bar{\epsilon}_{\boldsymbol{K}}-\mu\right) \phi_{\boldsymbol{K} \sigma}(\tau) \\
& +\int_{0}^{\beta} \mathrm{d} \tau \sum_{\boldsymbol{K} \sigma} \phi_{\boldsymbol{K} \sigma}^{*}(\tau) \int \mathrm{d} \tilde{\boldsymbol{k}} V_{\boldsymbol{K}}^{*}(\tilde{\boldsymbol{k}})\left[\frac{\partial}{\partial \tau}+\bar{\zeta}_{\boldsymbol{K}+\tilde{\boldsymbol{k}} \sigma}\right]^{-1} V_{\boldsymbol{K}}(\tilde{\boldsymbol{k}}) \phi_{\boldsymbol{K} \sigma}(\tau) \\
= & -\frac{1}{\beta} \sum_{\mathrm{i} \omega_{n}} \sum_{\boldsymbol{K} \sigma} \phi_{\boldsymbol{K} \sigma}^{*}\left(\mathrm{i} \omega_{n}\right)\left(\mathrm{i} \omega_{n}-\bar{\epsilon}_{\boldsymbol{K} \sigma}+\mu-\int \mathrm{d} \tilde{\boldsymbol{k}} \frac{\left|V_{\boldsymbol{K}}(\tilde{\boldsymbol{k}})\right|^{2}}{\mathrm{i} \omega_{n}-\zeta_{\boldsymbol{K}+\tilde{\boldsymbol{k}}}}\right) \phi_{\boldsymbol{K} \sigma}\left(\mathrm{i} \omega_{n}\right) .
\end{aligned}
$$

Eq. (3.29) shows that $S_{0}$ is indeed identical to eq. (3.25), if we define

$$
\Gamma_{\boldsymbol{K}}\left(\mathrm{i} \omega_{n}\right)=\int \mathrm{d} \tilde{\boldsymbol{k}} \frac{\left|V_{\boldsymbol{K}}(\tilde{\boldsymbol{k}})\right|^{2}}{\mathrm{i} \omega_{n}-\zeta_{\boldsymbol{K}+\tilde{\boldsymbol{k}}}} .
$$

Thus, although the bath does not enter the algorithm explicitly, it is nevertheless implicitly included in the effective bare Green function $\mathcal{G}_{\boldsymbol{K} \sigma}\left(\mathrm{i} \omega_{n}\right)$.

\subsection{Antiferromagnetic order}

So far, all quantities such as Green functions and self-energies were diagonal in momentum space, because the DCA preserved the translational symmetry of the lattice problem. Antiferromagnetic order breaks translational symmetry, leading to a doubling of the unit cell. This means that the first Brillouin zone correspondingly becomes smaller. Evaluating the mapping for the 3D cubic lattice, then results in the reduced zone shown in fig. 2.2a.

In order to explicitely break the full translational symmetry alongside with the $\mathrm{SU}(2)$ symmetry, one adds staggered magnetic field $h_{i}=h_{0} \mathrm{e}^{\mathrm{i} \boldsymbol{Q} \cdot \boldsymbol{r}_{i}}$ with $\boldsymbol{Q}=(\pi, \pi, \pi)$ to the Hamiltonian eq. (2.1) as

$$
H_{h}=H+\sum_{i} h_{i} m_{i}
$$


where $m_{i}=n_{i \uparrow}-n_{i \downarrow}$ is the spin polarization at lattice site $i$. One can then in principle study the properties as function of this staggered field. However, because such a field is of little experimental relevance, one is conventionally only interested in the limit $h_{0} \rightarrow 0$. If a finite polarization remains in this limit, we have found a state with spontaneous symmetry breaking.

In a real simulation, one usually proceeds slightly differently via adding a small field (in our case we chose $h_{0}=0.01$ ) in the intialization of the iteration process. The field is switched off after the first few iterations for subsequent iterations and the system is allowed to evolve freely. Eventually, the process will converge either to a solution with vanishing staggered magnetization $\left\langle m_{i}\right\rangle=0$, meaning that we are in a parameter regime where the thermodynamically stable state is a paramagnet, or else have $\left\langle m_{i}\right\rangle \neq 0$ and thus an antiferromagnetically ordered state.

In order to be able to include such a field in our simulations, we have to ensure that the cluster we use has the proper translational symmetry with respect to a double unit cell. These clusters are the bipartite clusters (cf. section 3.7.2). Since the DCA is formulated in momentum space, the broken translational symmetry introduces explicit non-diagonal elements in quantities like the Green function or the self-energy. Using

$$
G_{\sigma \boldsymbol{K}_{1}, \boldsymbol{K}_{1}}\left(\mathrm{i} \omega_{n}\right)=\frac{1}{N} \sum_{i j} \exp \left[\mathrm{i}\left(\boldsymbol{K}_{1} \cdot \boldsymbol{R}_{i}-\boldsymbol{K}_{2} \cdot \boldsymbol{R}_{j}\right)\right] G_{\sigma i j}\left(\mathrm{i} \omega_{n}\right)
$$

as extension of eq. (3.6) for Green functions with momenta $\boldsymbol{K}_{1} \neq \boldsymbol{K}_{2}$, the antiferromagnetic Green function can be represented by the $2 \times 2$ matrix

$$
\begin{aligned}
\mathbf{G}_{\sigma \boldsymbol{K}^{\prime}}\left(\mathrm{i} \omega_{n}\right) & :=\left(\begin{array}{ll}
G_{\sigma \boldsymbol{K}^{\prime}}^{00}\left(\mathrm{i} \omega_{n}\right) & G_{\sigma \boldsymbol{K}^{\prime}}^{01}\left(\mathrm{i} \omega_{n}\right) \\
G_{\sigma \boldsymbol{K}^{\prime}}^{10}\left(\mathrm{i} \omega_{n}\right) & G_{\sigma \boldsymbol{K}^{\prime}}^{11}\left(\mathrm{i} \omega_{n}\right)
\end{array}\right) \\
& :=\left(\begin{array}{cc}
G_{\sigma \boldsymbol{K}^{\prime}, \boldsymbol{K}^{\prime}}\left(\mathrm{i} \omega_{n}\right) & G_{\sigma \boldsymbol{K}^{\prime}, \boldsymbol{K}^{\prime}+\boldsymbol{Q}}\left(\mathrm{i} \omega_{n}\right) \\
G_{\sigma \boldsymbol{K}^{\prime}+\boldsymbol{Q}, \boldsymbol{K}^{\prime}}\left(\mathrm{i} \omega_{n}\right) & G_{\sigma \boldsymbol{K}^{\prime}+\boldsymbol{Q}, \boldsymbol{K}^{\prime}+\boldsymbol{Q}}\left(\mathrm{i} \omega_{n}\right)
\end{array}\right)
\end{aligned}
$$

where $\boldsymbol{K}^{\prime}$ is now an element of the reduced Brillouin zone depicted in fig. 2.2a. The symmetry relations $G_{\sigma \boldsymbol{K}^{\prime}}^{00}\left(\mathrm{i} \omega_{n}\right)=G_{\bar{\sigma} \boldsymbol{K}^{\prime}}^{11}\left(\mathrm{i} \omega_{n}\right)=-\left(G_{\sigma \boldsymbol{K}^{\prime}}^{11}\left(\mathrm{i} \omega_{n}\right)\right)^{*}=-\left(G_{\bar{\sigma} \boldsymbol{K}^{\prime}}^{00}\left(\mathrm{i} \omega_{n}\right)\right)^{*}$ and $G_{\sigma \boldsymbol{K}^{\prime}}^{10}\left(\mathrm{i} \omega_{n}\right)=G_{\sigma \boldsymbol{K}^{\prime}}^{01}\left(\mathrm{i} \omega_{n}\right)=G_{\bar{\sigma} \boldsymbol{K}^{\prime}}^{10}\left(\mathrm{i} \omega_{n}\right)=G_{\bar{\sigma} \boldsymbol{K}^{\prime}}^{01}\left(\mathrm{i} \omega_{n}\right)$ hold for the Green function as well as for the self-energy, which must be extracted by matrix inversions,

$$
\boldsymbol{\Sigma}_{\sigma \boldsymbol{K}^{\prime}}\left(\mathrm{i} \omega_{n}\right)=\mathcal{G}_{\sigma \boldsymbol{K}^{\prime}}\left(\mathrm{i} \omega_{n}\right)^{-1}-\mathbf{G}_{\sigma \boldsymbol{K}^{\prime}}\left(\mathrm{i} \omega_{n}\right)^{-1} .
$$

The self-consistency loop fig. 3.2 applies to the antiferromagnetic case as well, if one replaces all scalar Green functions and self-energies by their corresponding $2 \times 2$ matrices. Special attention is required for the coarse-grained Green function $\overline{\mathbf{G}}_{\sigma \boldsymbol{K}^{\prime}}\left(\mathrm{i} \omega_{n}\right)$. In the presence of a staggered magnetic field, it can be described by [109]

$$
\overline{\mathbf{G}}_{\sigma \boldsymbol{K}^{\prime}}\left(\mathrm{i} \omega_{n}\right)=\frac{1}{V} \int \mathrm{d} \tilde{\boldsymbol{k}}\left[\left(\begin{array}{lr}
\mathrm{i} \omega_{n}+\mu-\epsilon_{\boldsymbol{K}^{\prime}+\tilde{\boldsymbol{k}}} & h_{\sigma} / 2 \\
h_{\sigma} / 2 & \mathrm{i} \omega_{n}+\mu-\epsilon_{\boldsymbol{K}^{\prime}+\boldsymbol{Q}+\tilde{\boldsymbol{k}}}
\end{array}\right)-\boldsymbol{\Sigma}_{\boldsymbol{K}^{\prime}}\left(\mathrm{i} \omega_{n}\right)\right]^{-1}
$$

using $h_{\sigma}=h_{\uparrow / \downarrow}= \pm h_{0}$. 


\subsection{Finite-size scaling}

The DCA has several well-defined limits. It becomes exact in the limit of infinite cluster size $N_{\mathrm{c}} \rightarrow \infty$, when the effective cluster problem becomes identical to the original problem and the cluster Green function becomes the exact Green function of the complete system. At cluster size $N_{\mathrm{c}}=1$ the DCA reduces to the DMFT for a finite-dimensional system. In this limit, all quantities become local. Thus, the DCA can be regarded as an interpolation scheme between the DMFT and the exact solution. Moreover, it can be shown that the DCA is a systematic approximation in cluster size and possesses a well-defined finite-size scaling behavior. This offers the possibility to systematically extrapolate results for finite clusters to the infinite system limit.

The approximation performed by the DCA is to replace the exact Green function by its coarse-grained counterparts in diagrams of the generating functional $\Phi$. The relation between the coarse-grained Green function $\bar{G}_{\boldsymbol{K}}$ and the exact Green function $G_{\boldsymbol{k}}$ is given by (cf. eqs. (3.28) and (3.27))

$$
G_{\boldsymbol{k}}=\left[\bar{G}_{\boldsymbol{K}}^{-1}-\delta \epsilon_{\boldsymbol{K}}(\tilde{\boldsymbol{k}})-\delta \Sigma_{\boldsymbol{K}}(\tilde{\boldsymbol{k}})+\Gamma_{\boldsymbol{K}}\right]^{-1}
$$

defining $\Sigma_{\boldsymbol{k}}=\Sigma_{\boldsymbol{K}}+\delta \Sigma_{\boldsymbol{K}}(\tilde{\boldsymbol{k}})$. Since the diagrams in $\Phi$ are integrated over $\tilde{\boldsymbol{k}}$ and $\frac{1}{V} \int \mathrm{d} \tilde{\boldsymbol{k}} \delta \epsilon_{\boldsymbol{K}}(\tilde{\boldsymbol{k}})=0$ by definition, all contributions linear in $\delta \epsilon_{\boldsymbol{K}}(\tilde{\boldsymbol{k}})$ vanish. If we assume a simple cubic cluster of size $N_{\mathrm{c}}=L_{\mathrm{c}}^{3}$, the cluster momenta are $\boldsymbol{K}=\left(\frac{n_{1} \pi}{L_{\mathrm{c}}}, \frac{n_{2} \pi}{L_{\mathrm{c}}}, \frac{n_{3} \pi}{L_{\mathrm{c}}}\right)$ with $n_{1}, n_{2}, n_{3} \in\left\{-L_{\mathrm{c}}, \ldots, L_{\mathrm{c}}\right\}$. Using the simple dispersion eq. (2.3), the quantity $\delta \epsilon_{\boldsymbol{K}}(\tilde{\boldsymbol{k}})=\epsilon_{\boldsymbol{K}+\tilde{\boldsymbol{k}}}-\bar{\epsilon}_{\boldsymbol{K}}$ becomes

$$
\delta \epsilon_{\boldsymbol{K}}(\tilde{\boldsymbol{k}})=\sum_{i=1}^{3} \cos \left(\frac{n_{i} \pi}{L_{\mathrm{c}}}+\tilde{k}_{i}\right)-\frac{\pi}{L_{\mathrm{c}}} \sum_{i=1}^{3} \int \mathrm{d} \tilde{\boldsymbol{k}} \cos \left(\frac{n_{i} \pi}{L_{\mathrm{c}}}+\tilde{k}_{i}\right) .
$$

We observe, that $\delta \epsilon_{\boldsymbol{K}}(\tilde{\boldsymbol{k}}) \sim \mathcal{O}\left(1 / L_{\mathrm{c}}\right)$ for large $L_{\mathrm{c}}$. The hybridization function vanishes like $\delta \epsilon_{\boldsymbol{K}}^{2}(\tilde{\boldsymbol{k}})$ to leading order (cf. eq. (3.30)), hence the average hybridization per cluster site scales like

$$
\bar{\Gamma}=\frac{1}{N_{\mathrm{c}}} \sum_{\boldsymbol{K}} \Gamma_{\boldsymbol{K}} \sim \mathcal{O}\left(1 / L_{\mathrm{c}}^{2}\right)
$$

If we assume, that the correction to the self-energy $\delta \Sigma_{\boldsymbol{K}}(\tilde{\boldsymbol{k}})$ scales with the same or higher order than the hybridization function, we find that the DCA converges like $\Phi_{\mathrm{DCA}} \approx \Phi+\mathcal{O}\left(1 / L_{\mathrm{c}}^{2}\right)$. The same behavior can be expected from all other observables derived from $\Phi_{\mathrm{DCA}}$. This scaling relation was numerically verified for the 1D FalicovKimball model [110] and will be the foundation of the finite-size scaling employed in chapter 5 . 
The argument above was only given for a cubic cluster of length $L_{\mathrm{c}}$. We usually perform DCA simulations on clusters that do not possess a simple cubic shape. For this reason, we have to define an effective cluster length $L_{\mathrm{c}}=N_{\mathrm{c}}^{1 / 3}$. The next section deals with the details of selecting clusters for optimal finite-size scaling.

\subsection{Cluster selection}

The choice of an appropriate finite-size cluster is important, since its size is severely limited by computational restrictions. Early simulations of 2D spin models on finite lattices using exact diagonalization were usually performed on square-shaped tiles [132]. In order to overcome the limited number of useful tilings it was shown that parallelogram tiles of a certain kind could also produce useful results [64]. Moreover, BETTS et al. introduced criteria to judge the quality of finite clusters with periodic boundary conditions based on topological structure and geometrical shape and determined a set of optimized parallelogram-shaped tiles of the square lattice $[19,18]$. They demonstrated that these tiles show a much better finite-size scaling behavior than their simple square-shaped counterparts. Thus, they argued that topological perfection is more important than the conservation of the full point group symmetry of the lattice. The criteria were later applied to parallelepiped tilings of the simple cubic lattice [21, 92], the face-centered cubic lattice [172], and the body-centered cubic lattice [20]. We present the criteria for finding clusters with good finite-size scaling behavior in the following two sections.

\subsubsection{Topological imperfection}

A set of clusters with different sizes exhibit good finite-size scaling if each cluster represents a certain length scale closely connected to its size. A physical relevant length scale is the number of nearest-neighbor shells which are part of the cluster. A nearest-neighbor shell shall be defined in a way, that it reflects the topology of a model with nearest-neighbor interaction: Site $i$ is $k^{\text {th }}$-nearest neighbor to a site $j$ if $i$ and $j$ can only be connected by $k$ or more hopping processes between nearest neighbors. For example, each site on an infinite square lattice possesses 4 nearest neighbors, 8 second-nearest neighbors, 12 third-nearest neighbors, 16 fourth-nearest neighbors, etc.

A finite cluster can only incorporate a finite number of nearest-neighbor shells. It is possible that there are less sites in a specific shell than in the corresponding shell of the infinite lattice. A topologically perfect cluster fills up the lowest neighbor shells completely and possesses only one incomplete shell for its remaining sites. An example for a perfect 16 site cluster is shown in fig. 3.4b. The two lowest shells are completely filled. The third-nearest-neighbor shell inhabits the remaining three sites and is incomplete. All other shells are empty. A simulation of a model 

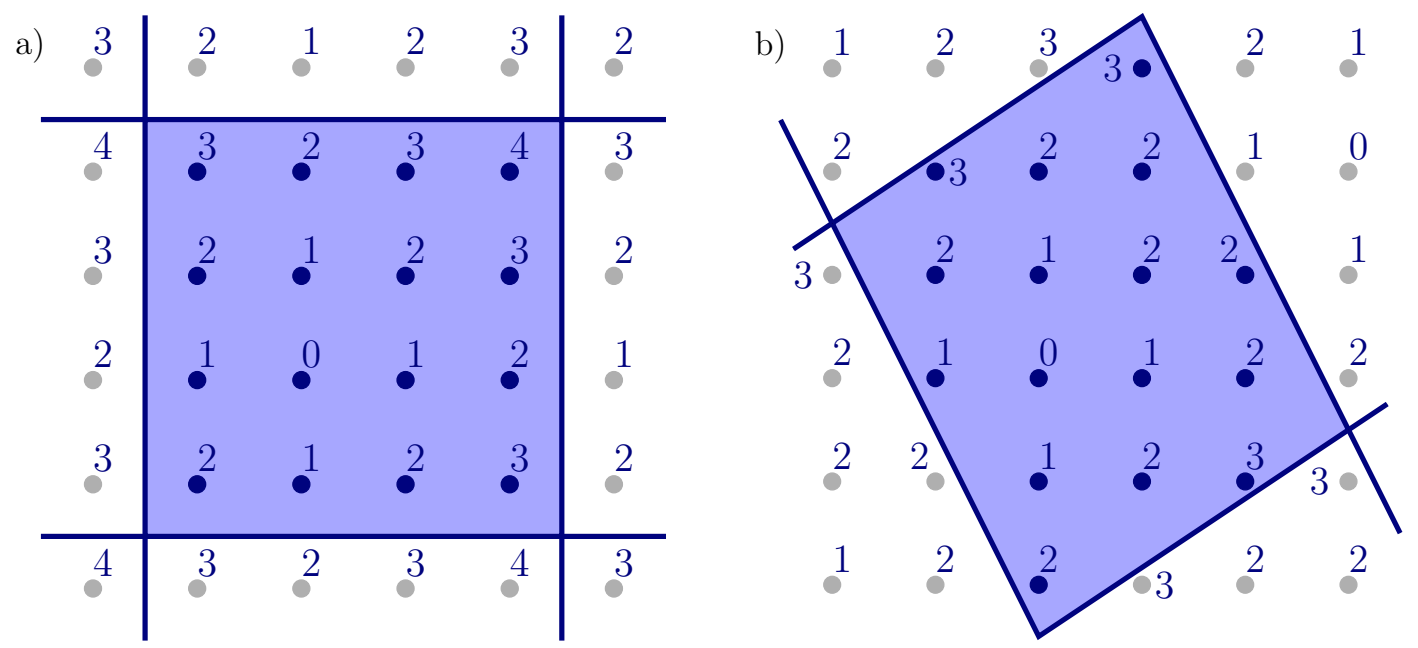

Figure 3.4: A comparison of two 16 site clusters in $2 \mathrm{D}$ with periodic boundary conditions which tile the simple square lattice. One arbitrary site (labeled by 0 ) is chosen as reference point. Nearest-neighbor shells are labeled by 1,2,3,4. The square cluster (a) exhibits a full nearest-neighbor shell and incomplete second (6 of 8 sites), third (4 of 12), and fourth-nearest-neighbor shells (1 of 16). Its topological imperfection is $I=3$. The parallelogram-shaped cluster (b) possesses complete nearest-neighbor and second-nearest-neighbor shells and only one incomplete third-nearest-neighbor shell (3 of 12). All higher neighbor shells are empty. Although the cluster (b) is not as symmetric as (a), it is topologically perfect $(I=0)$.

using this finite cluster correctly treats physical processes acting on a length scale up to the second-nearest-neighbor shell. The third-nearest-neighbor shell is treated approximately due to the lack of several sites. All higher length scales are not captured at all.

A less than perfect finite cluster is shown in fig. 3.4a. It exhibits incomplete second, third, and fourth-nearest-neighbor shells. This cluster treats even longer length scales (the fourth-nearest-neighbor shell) while approximating already the processes on the second-nearest-neighbor shell. It therefore mixes several different length scales and is not a pure representative of a specific scale. Although this cluster is a perfect square and conserves the point group symmetry of the lattice, it is less suitable for a systematic finite size analysis and should be avoided.

In order to have a quantitative statement of cluster perfection one defines the topological imperfection [21]

$$
I=I_{\mathrm{f}}-I_{\mathrm{p}} .
$$

The first quantity $I_{\mathrm{f}}$ is based on the cluster in question,

$$
I_{\mathrm{f}}=\sum_{k=0}^{\infty} k n_{k},
$$



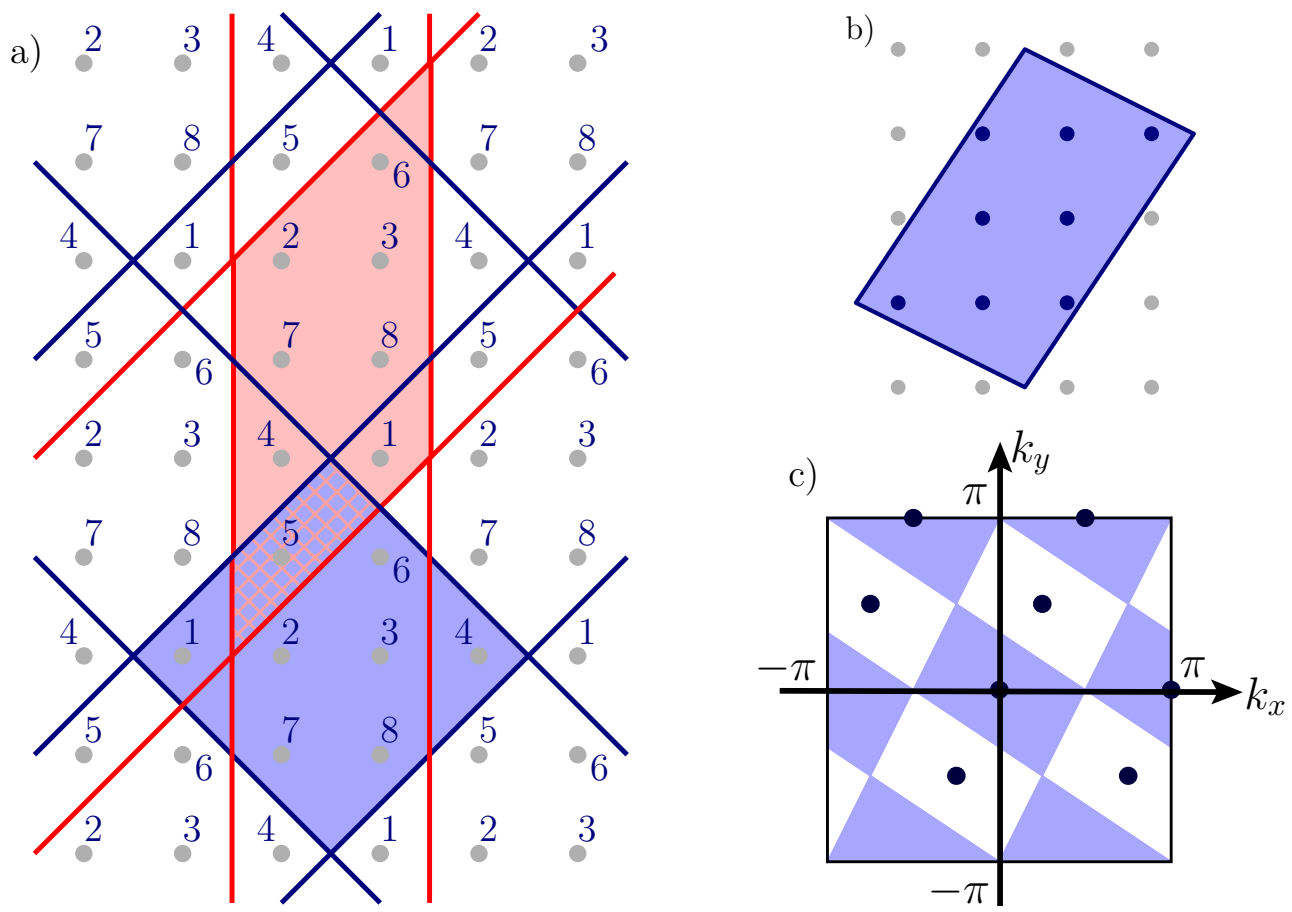

Figure 3.5: Panel a: The same cluster of fig. 3.1 described by the same parallelogram as in fig. 3.1 (blue) and by a completely different parallelogram (red). Each cluster site is identified by a number $1,2, \ldots, 8$. Right column: a non-bipartite cluster with even number of sites in real space (b) and in momentum space (c).

where $n_{k}$ is the number of sites in the $k^{\text {th }}$-nearest-neighbor shell. The second term $I_{\mathrm{p}}$ is the same quantity determined for a perfect cluster of the same size, which possesses at most one incomplete nearest-neighbor shell. This definition is independent of the fact, wether such a perfect cluster exists or not. The definition of $I$ can easily be extended to the simple cubic lattice in 3D. In this case the number of sites in the nearest-neighbor shells of the infinite lattice are $n_{1}=8, n_{2}=18, n_{3}=38$, etc.

\subsubsection{Geometry and symmetry}

The topological imperfection is taken to be the most important quantity to judge the quality of a cluster [21]. For a particular size, there exist many clusters with the same imperfection but described by different matrices $\mathbf{C}$. Some of these are even identical. Due to the periodic boundary conditions, the description by the matrix $\mathbf{C}$ is not unique. For example, the cluster of fig. 3.1 can be described with different parallelograms (cf. fig. 3.5a). It can be shown, that two clusters $\mathbf{C}_{1}$ and $\mathbf{C}_{2}$ of size $N_{\mathrm{c}}$ are identical, if and only if $\mathbf{A}=\mathbf{C}_{2}^{-1} \mathbf{C}_{1}$ has only integer elements [107]. But there can still exist several non-identical clusters with different geometrical properties including clusters with very thin, flat, or elongated shapes. These clusters are not 
desirable, since they exhibit effectively only two or even one-dimensional properties. It is therefore necessary to choose a cluster which is as close to a cubic shape as possible.

Using the lengths of the four body diagonals of the parallelepiped $d_{1}, d_{2}, d_{3}, d_{4}$ and of the six face diagonals $f_{1}, f_{2}, \ldots, f_{6}$, we take their geometric mean length $d=\left(d_{1} d_{2} d_{3} d_{4}\right)^{1 / 4}$ and $f=\left(f_{1} f_{2} f_{3} f_{4} f_{5} f_{6}\right)^{1 / 6}$, respectively, as well as the mean of the three edges $l=\left(l_{1} l_{2} l_{3}\right)^{1 / 3}$. These are used to define the cubicities [21]

$$
c_{1}=\frac{\sqrt{3} l}{d} \quad \text { and } \quad c_{2}=\frac{\sqrt{2} l}{f} .
$$

Obviously $c_{1}=c_{2}=1$ for the cube. The closer these cubicities are to unity the closer the parallelepiped is to a cube. The combined cubicity parameter

$$
c=\max \left(c_{1}, c_{1}^{-1}\right) \max \left(c_{2}, c_{2}^{-1}\right)
$$

is used as an criterion to choose clusters that are closest to a cube.

A parallelepiped cluster obviously breaks the cubic symmetry of the infinite lattice. While all clusters are at least symmetric under coordinate inversion, the remaining 46 mirror and rotation symmetries of the cubic group may be broken.

A potential antiferromagnetic order of a simulated system will be frustrated, if the lattice is not bipartite, i. e., cannot be split into two sub-lattices. The infinite simple cubic lattice is bipartite, but a finite cluster may not. All clusters with uneven number of sites are non-bipartite, but also certain clusters with an even number of sites. Thus, in order to avoid an artificial frustration, a bipartite cluster is mandatory for a study of antiferromagnetic order. Another disadvantage of nonbipartite lattices is, that they cause a sign problem in the QMC algorithm even in particle-hole-symmetric situations. The two-sub-lattice structure of a bipartite cluster transforms into momentum space in the following way: For each cluster momentum $\boldsymbol{K}$ the momentum $\boldsymbol{K}+(\pi, \pi, \pi)$ is also a cluster momentum. The cluster momenta can accordingly be divided into pairs. In particular the momentum $(\pi, \pi, \pi)$ itself is a cluster momentum. This symmetry is a necessary ingredient for the cancelation of the fermionic sign. Since the sign problem limits the performance of the algorithm severely, non-bipartite clusters should be avoided at least in the context of QMC simulations. The cluster of fig. 3.5b is a $2 \mathrm{D}$ example of a nonbipartite cluster with even number of sites. In $2 \mathrm{D}$ the vector $(\pi, \pi)$ is the relevant direction for antiferromagnetic order. If one observes the cluster in momentum space (fig 3.5c), the lack of the cluster momentum $(\pi, \pi)$ becomes apparent.

All simulations in this work exclusively used bipartite clusters which were optimized following these rules. For a given cluster size $N_{\mathrm{c}}$, the chosen cluster was the one with the smallest possible topological imperfection and cubicity closest to one, in this order of priority. Only bipartite clusters with an even number of cluster sites were used. For $N_{\mathrm{c}} \leq 100$, these clusters were already published in ref. [92]. 


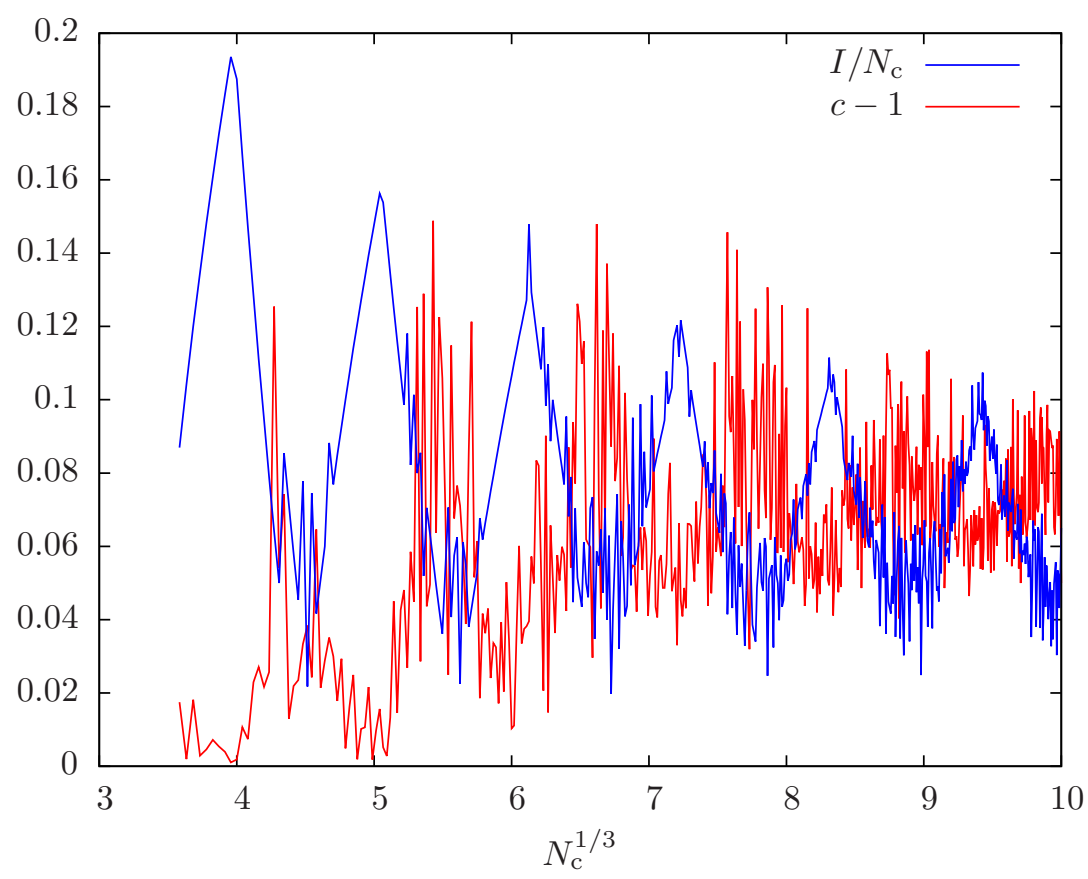

Figure 3.6: Imperfection per cluster size $I / N_{\mathrm{c}}$ and the deviation from cubic shape $c-1$ for optimized clusters of the $3 \mathrm{D}$ simple cubic lattice. Only bipartite clusters with $46<N_{\mathrm{c}}<1000$ and even number of sites are shown.

Additional bipartite clusters in 3D with $N_{\mathrm{c}} \leq 1000$ were found by examining all matrices $\mathbf{C}$ with individual components up to \pm 9 by direct enumeration. A selection is listed in appendix E. Fig. 3.6 shows imperfection and cubicity of these optimized clusters plotted against the effective cluster radius $N_{\mathrm{c}}^{1 / 3}$. A periodic pattern in the imperfection is clearly visible showing that minimizing $I$ effectively causes the nearest-neighbor shells to be filled up one after the other. Unfortunately, regions of low imperfection coincide with those of largest deviation from the cubic shape. However, the cubicity remains at moderate values $c<1.2$ and fluctuates heavily, so that individual clusters with low cubicity can be found.

\subsection{Summary}

The DCA provides an approximation scheme for the Hubbard model by approximating the infinite-lattice problem on an effective finite cluster embedded in a mean field. All quantum fluctuations local to the cluster are treated exactly while longranged correlations are included in a mean-field limit. The DCA is a systematic generalization to the DMFT and offers the possibility of a finite-size scaling analysis (cf. chapter 5 for an application). The remaining problem, i. e., how to solve the effective cluster problem, is the subject of the subsequent chapter. 


\section{Quantum Monte Carlo simulations in continuous time}

In this chapter, we give a detailed description of the QMC algorithms employed to solve the cluster model introduced in chapter 3. An introduction into QMC methods and an overview of Monte Carlo techniques in general is followed by a characterization of the two distinct continuous-time QMC algorithms used for this work.

There exist several completely different classes of algorithms which are commonly described by the term "quantum Monte Carlo". They only have in common that they employ stochastic methods to simulate quantum mechanical problems. Variational Monte Carlo [114, 33] and diffusion Monte Carlo [42] are used for the direct calculation of wave functions of many-body systems, while path integral Monte Carlo techniques [34] such as the stochastic series expansion [160, 177], the loop [41, 14, 40] and the worm algorithm $[143,142]$ sample the action of many-body-problems. This latter group of algorithms is mostly applied to bosonic systems [68, 162], quantum magnets [66, 158, 159], and one-dimensional fermionic systems [165]. The fermionic sign problem [184] generally prevents the application of these methods to fermionic models in higher dimensions.

A third group of algorithms provide methods suitable for the stochastic sampling of the action of fermions. Because of the sign problem, their application is limited to either particle-hole-symmetric lattice problems [22] or to impurity problems within an optional DMFT or DCA context. In the past, the standard method for fermionic impurity models was the algorithm by HIRSCH and FYE [75]. It decouples the fermion degrees of freedom by a discrete Hubbard-Stratonovich transformation $[73,74]$ of the partition function, which introduces an auxiliary Ising field. This transformation requires a discretization of the imaginary time axis into equally spaced time slices $\Delta \tau$ using a Trotter-Suzuki decomposition $[182,174]$. Such a discretization introduces a systematic error of $\mathcal{O}\left(\Delta \tau^{2}\right)$, which demands a careful and numerically expensive extrapolation $\Delta \tau \rightarrow 0$. A characteristic technical feature of the algorithm is that the Monte Carlo weights depend on the numerically often expensive calculation of determinants.

Alternatives to the Hirsch-Fye algorithm have been developed in recent years $[152,153,59,194,196,60]$. All of them avoid the error due to the Trotter-Suzuki decomposition and work in continuous time instead. They are similar to the HirschFye algorithm in this respect, that they require the calculation of determinants. 
We employ two of these algorithms, which are both based on an expansion in the interaction term of the Hamiltonian. The continuous-time interaction expansion algorithm (CT-INT) was developed by RuBTsov et al. [151, 152, 153]. It is often called "weak coupling" algorithm [119, 58, 59], which is somewhat inappropriate, since its application is not limited to weak coupling strengths. The continuoustime auxiliary-field method (CT-AUX) was initially developed by GuLL et al. [59] based on an algorithm for finite lattices by Rombouts et al. [148, 149]. This method exhibits stronger similarities to the Hirsch-Fye algorithm than the CT-INT. Although technically different, both algorithms were shown to be equivalent for Hubbard type interactions [119]. There exists another algorithm also formulated in continuous imaginary time, which is based on an expansion in the hybridization with the surrounding bath [194, 196]. However, it is not suitable for large scale cluster applications, because the interacting cluster problem has to be solved exactly. This causes an exponential scaling of the algorithm with cluster size [196], which limits the size of treatable clusters to a minimum.

\subsection{Monte Carlo evaluation}

We are aiming at a Monte Carlo evaluation of the single particle Green function and other thermodynamic observables. The thermal expectation value of an observable $O$ is

$$
\langle O\rangle=\frac{1}{Z} \operatorname{Tr} O \mathrm{e}^{-\beta H_{\mathrm{c}}}
$$

with the partition function

$$
Z=\operatorname{Tr} \mathrm{e}^{-\beta H_{\mathrm{c}}}
$$

of the effective cluster model eq. (3.31). In a many-body quantum system, the direct evaluation of the trace over the exponentially large number of many-particle states is generally impossible. In order to evaluate eq. (4.1) stochastically, the thermal average needs to be transformed into a sum of configurations $C$ weighted by $w(C)$,

$$
\langle O\rangle=\sum_{C} O(C) w(C)
$$

Such a weighted average can be calculated by a Monte Carlo procedure. The explicite calculation of the weights $w(C)$ themselves is generally not possible, since the norm of the weights is determined by the partition function $Z$ whose calculation again involves a trace over an exponentially large number of states. Another exponentially hard effort is the diagonalization of the Hamiltonian $H_{\mathrm{c}}$, which could otherwise provide the transformation eq. (4.3). The details of the transformation are specific to each QMC algorithm and will be covered in sections 4.2 and 4.3, respectively. The inability to calculate $Z$ directly also prohibits the direct Monte 
Carlo evaluation of observables that depend directly on the partition function, for example the entropy $S=\frac{1}{\beta} \ln Z$ or the free energy.

In order to sample averages like eq. (4.3) effectively, we apply Monte Carlo techniques [99]. We generate a Markov chain of configurations

$$
C_{1} \rightarrow C_{2} \rightarrow \cdots \rightarrow C_{n} \rightarrow C_{n+1} \rightarrow \ldots \text {. }
$$

A configuration $C_{n+1}$ in the chain is only connected to its immediate predecessor $C_{n}$ by the transition probability $t\left(C_{n} \rightarrow C_{n+1}\right)$. That means, that the current configuration of the chain does not depend on its past nor do any future configurations. We need to find transition probabilities that will generate a finite set of configurations $C_{1}, C_{2}, \ldots, C_{N}$, such that these configurations are distributed according to $w(C)$ in the limit $N \rightarrow \infty$. If this can be accomplished, the average eq. (4.3) is approximated by

$$
\langle O\rangle=\sum_{n=1}^{\infty} O\left(C_{n}\right) \approx \sum_{n=1}^{N} O\left(C_{n}\right)+\mathcal{O}(\sqrt{1 / N}) .
$$

The statistical error of such an approximation is of order $\mathcal{O}(\sqrt{1 / N})$. Thus, in principle every observable can be calculated with a desired accuracy by a sufficiently large number of generated configurations. The Monte Carlo estimate for $\langle O\rangle$ possesses statistical errors only and is otherwise exact.

The Markov chain must generate configurations distributed by $w(C)$. It is sufficient for the transition probabilities $t\left(C_{n} \rightarrow C_{n+1}\right)$ to fulfill two conditions:

Ergodicity: Any configuration must be reachable from any other configuration in a finite number of Markov steps.

Detailed balance: We demand

$$
t\left(C_{n} \rightarrow C_{n+1}\right) w\left(C_{n}\right)=t\left(C_{n+1} \rightarrow C_{n}\right) w\left(C_{n+1}\right) .
$$

We apply a Metropolis-Hastings algorithm $[117,67]$ to satisfy these conditions. The transition probability is split into a proposal part $p$ and an acceptance probability $a$,

$$
t\left(C_{n} \rightarrow C_{n+1}\right)=p\left(C_{n} \rightarrow C_{n+1}\right) a\left(C_{n} \rightarrow C_{n+1}\right) .
$$

Thus, the detailed balance condition becomes

$$
\frac{a\left(C_{n} \rightarrow C_{n+1}\right)}{a\left(C_{n+1} \rightarrow C_{n}\right)}=\frac{w\left(C_{n+1}\right) p\left(C_{n+1} \rightarrow C_{n}\right)}{w\left(C_{n}\right) p\left(C_{n} \rightarrow C_{n+1}\right)},
$$

which is satisfied, if we propose a change of the current configuration $C_{n}$ and accept the change with the probability

$$
a\left(C_{n} \rightarrow C_{n+1}\right)=\min \left(1, \frac{w\left(C_{n+1}\right) p\left(C_{n+1} \rightarrow C_{n}\right)}{w\left(C_{n}\right) p\left(C_{n} \rightarrow C_{n+1}\right)}\right) .
$$

Here the weights $w(C)$ only appear in ratios eliminating the dependence on the normalization $Z$. 


\subsubsection{Sign problem}

The interpretation of eq. (4.9) as a probability requires its non-negativity. While the proposal probabilities are usually positive, the weights stemming from eq. (4.3) are generally not. Depending on the QMC algorithm and the model in question the quantities $w(C)$ may be negative, thus inhibiting their interpretation as probabilities in a weighted sum. In principle, this problem can be avoided by switching from an average $\langle\ldots\rangle_{w}$ using $w(C)$ to $\langle\ldots\rangle_{|w|}$ involving the absolute values $|w(C)|$ of the weights. The relation

$$
\langle O\rangle_{w}=\frac{\sum_{C} O(C) w(C)}{\sum_{C} w(C)}=\frac{\sum_{C} \operatorname{sgn}(w(C)) O(C)|w(C)| / \sum_{C}|w(C)|}{\sum_{C} \operatorname{sgn}(w(C))|w(C)| / \sum_{C}|w(C)|}=\frac{\langle\operatorname{sgn}(w) O\rangle_{|w|}}{\langle\operatorname{sgn}(w)\rangle_{|w|}}
$$

displays that the average of each observable $O$ can be calculated by measuring $\langle\operatorname{sgn}(w) O\rangle_{|w|}$ with respect to the positive weights $|w(C)|$ and subsequently dividing by the average sign of the weights $\langle\operatorname{sgn}(w)\rangle_{|w|}$. This ensures that the Monte Carlo algorithm described in the previous section is still applicable. Numerical problems arise if the average sign becomes very small, which is unfortunately often the case. The statistical errors even grow exponentially with $\beta$ and system size $N_{\mathrm{c}}$, which renders the measurement of an observable with vanishing sign an exponentially hard problem [184]. This can be shown, by observing that the average sign $\langle\operatorname{sgn}(w)\rangle_{|w|}=$ $Z_{w} / Z_{|w|}$ is the ratio of the partition functions of the system with respect to $w$, $Z_{w}=\sum_{C} w(C)$, and with respect to $|w|, Z_{|w|}=\sum_{C}|w(C)|$. Per definition, the partition function depends exponentially on the free energy $F=-\frac{1}{\beta} \ln Z$. That means that also the average sign depends exponentially on the difference $\Delta f$ of the free energy densities $f=F / N_{\mathrm{c}}$, i. e.,

$$
\langle\operatorname{sgn}(w)\rangle_{|w|} \propto \exp \left(-\beta N_{\mathrm{c}} \Delta f\right)
$$

The relative error $\sigma(\operatorname{sgn}) /\langle\operatorname{sgn}\rangle_{|w|}$ follows as

$$
\frac{\sigma(\operatorname{sgn})}{\langle\operatorname{sgn}\rangle}=\frac{\sqrt{\left(\left\langle\operatorname{sgn}^{2}\right\rangle_{|w|}-\langle\operatorname{sgn}\rangle_{|w|}^{2}\right) / N}}{\langle\operatorname{sgn}\rangle_{|w|}} \propto \frac{\mathrm{e}^{\beta N_{\mathrm{C}} \Delta f}}{\sqrt{N}} .
$$

Since measurements of all observables depend on the average sign, the exponential increase of the errors with inverse temperature and system size is true for all observables. This effect is commonly known as the sign problem. The exponential increase of the statistical errors effectively prevents the application of QMC techniques to many models. 


\subsection{Continuous-time interaction expansion}

The CT-INT algorithm provides a numerical tractable transformation of the expectation value eq. (4.1) into the weighted sum eq. (4.3) by expanding the partition function in the interaction term of the Hamiltonian.

We divide the cluster Hamiltonian $H_{\mathrm{c}}=H_{0}+H_{U}$ into a non-interacting part $H_{0}$ and

$$
H_{U}=U \sum_{i} n_{i \uparrow} n_{i \downarrow}
$$

We switch into the interaction representation by defining time-dependent operators $O(\tau)$ with respect to $H_{0}$, i. e.,

$$
O(\tau)=\mathrm{e}^{\tau H_{0}} O \mathrm{e}^{-\tau H_{0}}
$$

If we introduce the operator

$$
U(\beta)=\mathrm{e}^{\beta H_{0}} \mathrm{e}^{-\beta H_{\mathrm{c}}},
$$

the partition function eq. (4.2) can be written as

$$
Z=\operatorname{Tr}\left[\mathrm{e}^{-\beta H_{0}} U(\beta)\right] .
$$

The operator $U(\beta)$ has the property

$$
\frac{\mathrm{d} U(\beta)}{\mathrm{d} \beta}=-H_{U}(\beta) U(\beta) \Rightarrow U(\beta)=T_{\tau} \exp \left[-\int_{0}^{\beta} \mathrm{d} \tau H_{U}(\tau)\right] .
$$

The partition function in the interaction representation follows as

$$
Z=\operatorname{Tr}\left[\mathrm{e}^{-\beta H_{0}} T_{\tau} \mathrm{e}^{-\int_{0}^{\beta} \mathrm{d} \tau H_{U}(\tau)}\right]=Z_{0}\left\langle\mathrm{e}^{-\int_{0}^{\beta} \mathrm{d} \tau H_{U}(\tau)}\right\rangle_{0},
$$

which defines the non-interacting partition function

$$
Z_{0}=\operatorname{Tr} T_{\tau} \mathrm{e}^{-\beta H_{0}}
$$

and the ensemble average

$$
\langle\ldots\rangle_{0}=\frac{1}{Z_{0}} \operatorname{Tr} T_{\tau} \ldots \mathrm{e}^{-\beta H_{0}} .
$$

The interaction Hamiltonian $H_{U}$ is modified by coupling an auxiliary Ising field $s_{i}= \pm 1$ to each cluster site. Thus, eq. (4.13) becomes

$$
H_{U}=U \sum_{i} \sum_{s_{i}= \pm 1}\left(n_{i \uparrow}-\frac{1}{2}-\delta s_{i}\right)\left(n_{i \downarrow}-\frac{1}{2}+\delta s_{i}\right) \text {. }
$$


The coupling strength $\delta$ and the additional terms $\frac{1}{2}$ cause no physical change in the Hamiltonian except a trivial shift in energy. However, the modification greatly affects the sign of the Monte Carlo weights and can even eliminate the sign problem for particle hole symmetric models [151, 6].

An expansion of the exponential function in the partition function eq. (4.18) to all orders yields

$$
\begin{aligned}
Z= & Z_{0} \sum_{k=0}^{\infty}(-U)^{k} \int_{0}^{\beta} \mathrm{d} \tau_{1} \cdots \int_{0}^{\tau_{k-1}} \mathrm{~d} \tau_{k} \sum_{i_{1} s_{i_{1}}} \cdots \sum_{i_{k} s_{i_{k}}} \\
& \left\langle\left(n_{i_{1} \uparrow}\left(\tau_{1}\right)-\alpha_{1 \uparrow}\right)\left(n_{i_{1} \downarrow}\left(\tau_{1}\right)-\alpha_{1 \downarrow}\right) \ldots\left(n_{i_{k} \uparrow}\left(\tau_{k}\right)-\alpha_{k \uparrow}\right)\left(n_{i_{k} \downarrow}\left(\tau_{k}\right)-\alpha_{k \downarrow}\right)\right\rangle_{0} .
\end{aligned}
$$

Here we have defined $\alpha_{k \sigma}=\frac{1}{2}+\operatorname{sgn}(\sigma) \delta s_{i_{k}}\left(\tau_{k}\right)$ with $\operatorname{sgn}(\uparrow)=1$ and $\operatorname{sgn}(\downarrow)=-1$. Since the average $\langle\ldots\rangle_{0}$ is performed over a non-interacting system, we can employ Wick's theorem [198] to factorize the expectation values in this expression. The result is a sum over all possible contractions of the operator string in eq. (4.22), which can be expressed in terms of the determinant of a $2 k \times 2 k$ matrix. This determinant factorizes into two sub-determinants for each spin resulting in

$$
Z=Z_{0} \sum_{k=0}^{\infty}(-U)^{k} \int_{0}^{\beta} \mathrm{d} \tau_{1} \cdots \int_{0}^{\tau_{k-1}} \mathrm{~d} \tau_{k} \sum_{i_{1} s_{i_{1}}} \cdots \sum_{i_{k} s_{i_{k}}} \prod_{\sigma} \operatorname{det}\left[G_{k \sigma}^{0}-A_{k \sigma}\right]
$$

with

$$
A_{k \sigma}=\operatorname{diag}\left(\alpha_{1 \sigma}, \alpha_{2 \sigma}, \ldots, \alpha_{k \sigma}\right) \text {. }
$$

Since $\left\langle c_{i \sigma}(\tau) c_{j \sigma}^{\dagger}\left(\tau^{\prime}\right)\right\rangle_{0}=-\mathcal{G}_{i j \sigma}\left(\tau-\tau^{\prime}\right)$ and $\left\langle n_{i \sigma}(\tau)\right\rangle_{0}=-\mathcal{G}_{i i \sigma}\left(\beta^{-}\right)$, the matrix $G_{k \sigma}^{0}$ can be explicitly evaluated yielding

$$
G_{k \sigma}^{0}=-\left(\begin{array}{cccc}
\mathcal{G}_{i_{1} i_{1} \sigma}\left(\beta^{-}\right) & \mathcal{G}_{i_{1} i_{2} \sigma}\left(\tau_{1}-\tau_{2}\right) & \cdots & \mathcal{G}_{i_{1} i_{k} \sigma}\left(\tau_{1}-\tau_{k}\right) \\
\mathcal{G}_{i_{2} i_{1} \sigma}\left(\tau_{2}-\tau_{1}\right) & \mathcal{G}_{i_{2} i_{2} \sigma}\left(\beta^{-}\right) & & \mathcal{G}_{i_{2} i_{k} \sigma}\left(\tau_{2}-\tau_{k}\right) \\
\vdots & & \ddots & \vdots \\
\mathcal{G}_{i_{k} i_{1} \sigma}\left(\tau_{k}-\tau_{1}\right) & \mathcal{G}_{i_{k} i_{2} \sigma}\left(\tau_{k}-\tau_{2}\right) & \cdots & \mathcal{G}_{i_{k} i_{k} \sigma}\left(\beta^{-}\right)
\end{array}\right)
$$

We can finally express the partition function eq. (4.23) as a sum of configurations $C$. Each configuration is characterized by an expansion order $k$ and a set of times, sites, and Ising field configurations,

$$
C=\left\{k ; i_{1}, \ldots, i_{k} ; \tau_{1}, \ldots, \tau_{k} ; s_{i_{1}}\left(\tau_{1}\right), \ldots, s_{i_{k}}\left(\tau_{k}\right)\right\} .
$$

Each one is weighted by

$$
w(C)=Z_{0}(-U)^{k} \prod_{\sigma} \operatorname{det}\left[G_{k \sigma}^{0}-A_{k \sigma}\right]
$$


such that the partition function eq. (4.23) can be written in the form

$$
Z=\sum_{C} w(C)
$$

if we formally combine all sums and integrals into a single sum over $C$. Accordingly, all observable averages can be expressed as the Monte Carlo average eq. (4.3). Again, we point out the importance of the matrix $A_{k \sigma}$ to avoid the sign problem. If one omitted $A_{k \sigma}, G_{k \uparrow}^{0}=G_{k \downarrow}^{0}$ would hold for spin-symmetric problems. The alternating sign stemming from the term $(-U)^{k}$ would then create a massive sign problem.

\subsubsection{Monte Carlo updates}

The presented QMC algorithm produces weights of the form (cf. eq. (4.27))

$$
w(C)=Z_{0} c^{k} \prod_{\sigma} \operatorname{det} M_{k \sigma}^{-1}
$$

with a constant $c=-U$ and $k \times k$ matrices $M_{k \sigma}^{-1}=G_{k \sigma}^{0}-A_{k \sigma}$. If the current configuration $C_{k}$ consists of $k$ vertices, a possible update is the insertion or removal of one vertex, thereby expanding or reducing the expansion order $k$ by one. The proposal probabilities for the insertion update is

$$
p\left(C_{k} \rightarrow C_{k+1}\right)=\frac{1}{\beta N_{\mathrm{c}}},
$$

which reflects the choice of randomly picking a time $\tau_{k+1}$ from the interval $[0, \beta]$ and a cluster site $s_{k+1}$ from $N_{c}$ possible sites. When removing one vertex from a configuration of order $k+1$, we must choose one of $k+1$ vertices. The proposal probability is consequently

$$
p\left(C_{k+1} \rightarrow C_{k}\right)=\frac{1}{k+1} .
$$

These two quantities enter the acceptance probability eq. (4.9), hence

$$
\begin{aligned}
& a\left(C_{k} \rightarrow C_{k+1}\right)=\min \left(1, \frac{c \beta N_{\mathrm{c}}}{k+1} \prod_{\sigma} \frac{\operatorname{det} M_{k+1, \sigma}^{-1}}{\operatorname{det} M_{k \sigma}^{-1}}\right), \\
& a\left(C_{k} \rightarrow C_{k-1}\right)=\min \left(1, \frac{k}{c \beta N_{\mathrm{c}}} \prod_{\sigma} \frac{\operatorname{det} M_{k-1, \sigma}^{-1}}{\operatorname{det} M_{k \sigma}^{-1}}\right) .
\end{aligned}
$$

Appendix A deals with the numerically efficient evaluation of these weights as well as with details of the update process of the matrix $M_{k \sigma}$. Updates of this kind are already sufficient to render the algorithm ergodic. In order to reach one arbitrary 


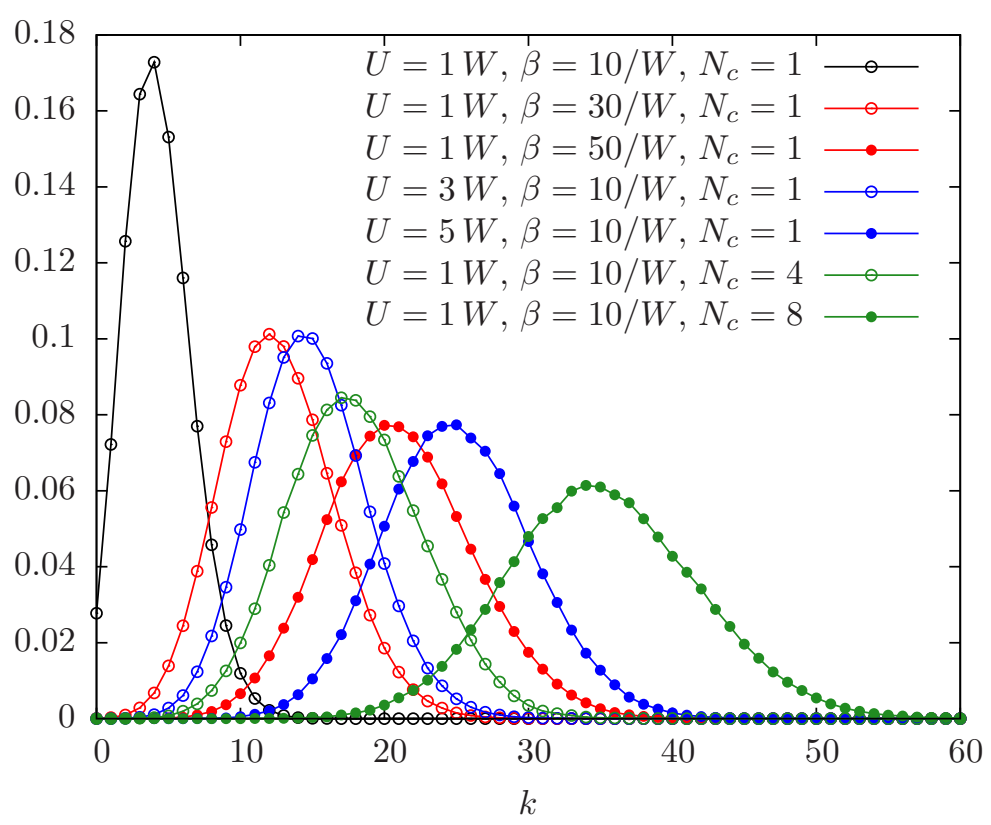

Figure 4.1: Histogram of the expansion order $k$ of several DCA simulations for the Hubbard model at half filling on a simple cubic lattice.

configuration from another, one could, for example, remove all current vertices and add the ones from the target configuration. The expansion order $k$ of the configuration can in principle reach all possible values. In real simulations, however, the average expansion order will be peaked around a finite value. Fig. 4.1 shows a histogram of the expansion order for several different parameter values. Each histogram exhibits a clear maximum and an overall Gaussian shape around the maximum. We can also observe that the average expansion order scales linearly with the inverse temperature $\beta$, the Coulomb repulsion $U$, and the cluster size $N_{\mathrm{c}}$. Appendix A shows that the Monte Carlo updates scale as $\mathcal{O}\left(k^{3}\right)$. The overall scaling of the algorithm is thus $\mathcal{O}\left(\left(\beta U N_{\mathrm{c}}\right)^{3}\right)$. That means that the simulation becomes more and more inefficient for increasing $\beta, U$, and $N_{\mathrm{c}}$.

\subsubsection{Observables}

In the DCA context, the quantity we are most interested in is the single-particle Green function as defined in eqs. (3.5) and (3.6). The imaginary-time dependent Green function in real space is easily expressed in the interaction picture by

$$
G_{\sigma i j}\left(\tau-\tau^{\prime}\right)=-\left\langle T_{\tau} c_{i \sigma}(\tau) c_{j \sigma}^{\dagger}\left(\tau^{\prime}\right)\right\rangle=\frac{\left\langle-\mathrm{e}^{-\int_{0}^{\beta} \mathrm{d} \tau H_{U}(\tau)} c_{i \sigma}(\tau) c_{j \sigma}^{\dagger}\left(\tau^{\prime}\right)\right\rangle_{0}}{\left\langle\mathrm{e}^{-\int_{0}^{\beta} \mathrm{d} \tau H_{U}(\tau)}\right\rangle_{0}}
$$


Applying the expansion eq. (4.22) to the enumerator yields a sum over weights

$$
\tilde{w}(C)=Z_{0} c^{k} \operatorname{det} \tilde{M}_{k+1, \sigma}^{-1} \operatorname{det} M_{k \bar{\sigma}}^{-1},
$$

where $\operatorname{det} \tilde{M}_{k+1, \sigma}^{-1}$ denotes the original $k \times k$ matrix $M_{k \sigma}^{-1}=G_{k \sigma}^{0}-A_{k \sigma}$ augmented by an additional row and column,

$$
\tilde{M}_{k+1, \sigma}^{-1}=\left(\begin{array}{cc}
\left(M_{k \sigma}^{-1}\right)_{p q} & \mathcal{G}_{\sigma i_{p} j}\left(\tau_{p}-\tau^{\prime}\right) \\
\mathcal{G}_{\sigma i i_{q}}\left(\tau-\tau_{q}\right) & \mathcal{G}_{\sigma i j}\left(\tau-\tau^{\prime}\right)
\end{array}\right)
$$

The indices $p$ and $q$ are elements of $1,2, \ldots, k$. Thus,

$$
G_{\sigma i j}\left(\tau-\tau^{\prime}\right)=\frac{\sum_{C} \frac{\tilde{w}(C)}{w(C)} w(C)}{\sum_{C} w(C)}=\left\langle\frac{\operatorname{det} \tilde{M}_{k+1, \sigma}^{-1}}{\operatorname{det} M_{k \sigma}^{-1}}\right\rangle_{w}=\left\langle\frac{\operatorname{det} M_{k, \sigma}}{\operatorname{det} \tilde{M}_{k+1, \sigma}}\right\rangle_{w}
$$

which exhibits a similar ratio of determinants as the Monte Carlo weight eq. (4.32). Applying the framework detailed in appendix A (cf. eq. (A.8)), the Green function becomes

$$
G_{\sigma i j}\left(\tau-\tau^{\prime}\right)=\mathcal{G}_{\sigma i j}\left(\tau-\tau^{\prime}\right)-\left\langle\sum_{p, q=1}^{k} \mathcal{G}_{\sigma i i_{p}}\left(\tau_{p}-\tau^{\prime}\right)\left(M_{k \sigma}\right)_{p q} \mathcal{G}_{\sigma i_{q} j}\left(\tau-\tau_{q}\right)\right\rangle_{w}
$$

A spatial and temporal Fourier transformation into Matsubara frequencies further simplifies this expression yielding

$$
G_{\sigma \boldsymbol{K}}\left(\mathrm{i} \omega_{n}\right)=\mathcal{G}_{\sigma \boldsymbol{K}}\left(\mathrm{i} \omega_{n}\right)-\mathcal{G}_{\sigma \boldsymbol{K}}^{2}\left(\mathrm{i} \omega_{n}\right) \frac{1}{\beta}\left\langle\sum_{p, q=1}^{k} \mathrm{e}^{\mathrm{i} \boldsymbol{K} \cdot\left(\boldsymbol{R}_{i_{p}}-\boldsymbol{R}_{i_{q}}\right)} \mathrm{e}^{\mathrm{i} \omega_{n}\left(\tau_{p}-\tau_{q}\right)}\left(M_{k \sigma}\right)_{p q}\right\rangle_{w}
$$

for paramagnetic systems. Antiferromagnetic Green function are no longer diagonal in momentum space. The spatial Fourier transformation of eq. (4.38) leads to the matrix notation of eq. (3.39),

$$
\boldsymbol{G}_{\sigma \boldsymbol{K}^{\prime}}\left(\mathrm{i} \omega_{n}\right)=\mathcal{G}_{\sigma \boldsymbol{K}^{\prime}}\left(\mathrm{i} \omega_{n}\right)-\mathcal{G}_{\sigma \boldsymbol{K}^{\prime}}\left(\mathrm{i} \omega_{n}\right)\left\langle\boldsymbol{M}_{k \sigma}\right\rangle_{w} \mathcal{G}_{\sigma \boldsymbol{K}^{\prime}}\left(\mathrm{i} \omega_{n}\right)
$$

using the $2 \times 2$ matrix

$$
\left(\boldsymbol{M}_{k \sigma}\right)_{\boldsymbol{K}_{1} \boldsymbol{K}_{2}}=\frac{1}{\beta} \sum_{p, q=1}^{k} \mathrm{e}^{\mathrm{i}\left(\boldsymbol{K}_{1} \cdot \boldsymbol{R}_{i_{p}}-\boldsymbol{K}_{2} \cdot \boldsymbol{R}_{i_{q}}\right)} \mathrm{e}^{\mathrm{i} \omega_{n}\left(\tau_{p}-\tau_{q}\right)}\left(M_{k \sigma}\right)_{p q} .
$$

In contrast to the Hirsch-Fye algorithm, the Green function can be directly measured in momentum space and Matsubara frequencies. A Monte Carlo measurement consists of the evaluation of the quantity in the brackets $\langle\ldots\rangle_{w}$, which is basically a spatial and temporal Fourier transformation of the configuration matrix $M_{k \sigma}$. The 


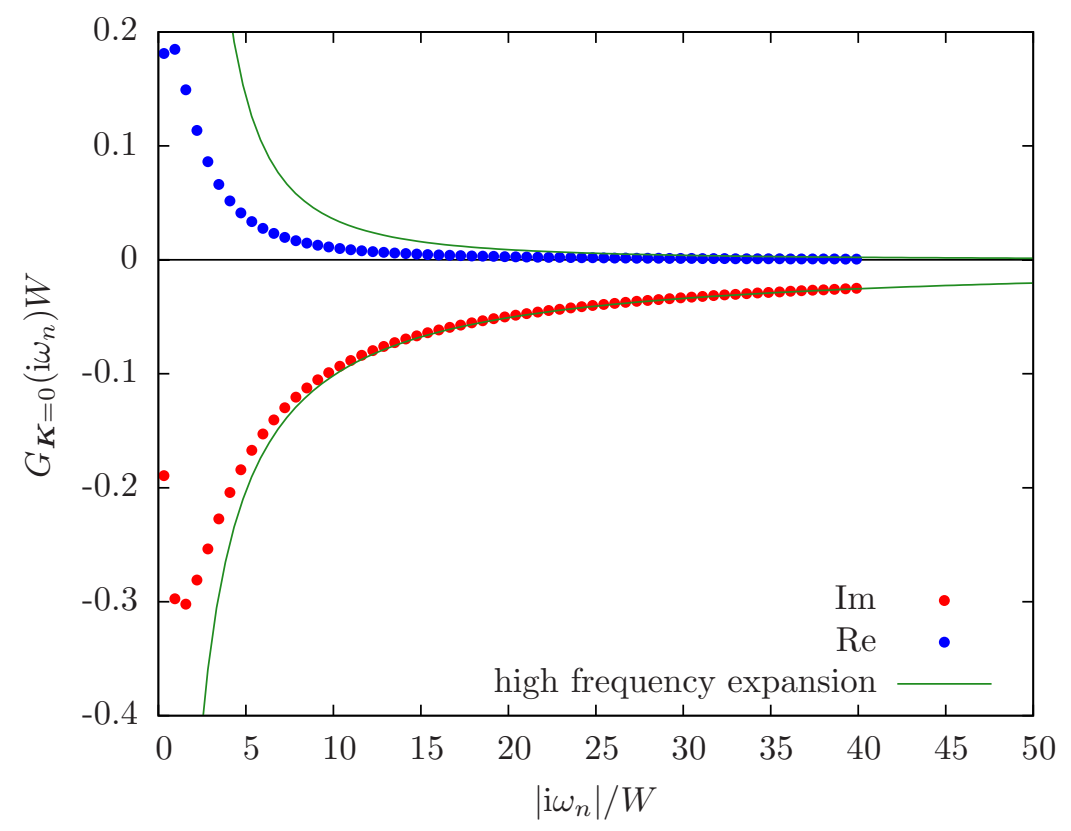

Figure 4.2: The paramagnetic single-particle Green function $G_{\boldsymbol{K}=0}\left(\mathrm{i} \omega_{n}\right)$ of the Hubbard model on the simple cubic lattice at half filling. The Green function was calculated in a DCA simulation with parameters $N_{\mathrm{c}}=18, U=W$, and $T=$ $0.025 \mathrm{~W}$. Points denote QMC measurements using the CT-INT algorithm. The error bars are smaller than the symbols. Solid lines denote the solution of a high-frequency expansion (cf. appendix B).

calculation must be repeated for each Matsubara frequency. Since one is naturally limited to measurements of a finite number of frequencies, only a certain number of lowest frequencies is measured. The high-frequency behavior can be approximated by a series expansion (cf. appendix B). The number of measured frequencies depends on the parameters of the simulation. Fig. 4.2 shows a Green function as calculated by the CT-INT algorithm. In this example, the high-frequency expansion is valid for $\left|i \omega_{n}\right| \gtrsim 30$. Measurements of higher Matsubara frequencies are therefore unnecessary.

The local particle density $\left\langle n_{\sigma i}\right\rangle$ and the spin polarization $m_{i}=\left\langle n_{\uparrow i}-n_{\downarrow i}\right\rangle$ are easily measured using eq. (4.38) by evaluating $n_{\sigma i}=1+G_{\sigma i i}\left(\tau_{\text {ref }}-\tau_{\text {ref }}\right)$, where the reference time $\tau_{\text {ref }}$ is chosen randomly.

For measuring two-particle correlators such as the density-density correlations $\left\langle n_{i \sigma} n_{j \sigma^{\prime}}\right\rangle$, the matrix $M_{k \sigma}$ must be extended by two additional rows and columns. Applying Wick's theorem, the result is the determinant of a $2 \times 2$ matrix,

$$
\left\langle n_{i \sigma} n_{j \sigma^{\prime}}\right\rangle=\left\langle\operatorname{det}\left(\begin{array}{cc}
n_{i \sigma} & G_{\sigma \sigma^{\prime} i j}\left(0^{+}\right) \\
G_{\sigma^{\prime} \sigma j i}\left(0^{+}\right) & n_{j \sigma^{\prime}}
\end{array}\right)\right\rangle_{w} .
$$

For the spin-symmetric Hubbard model, the off-diagonal elements are non-zero for 
$\sigma=\sigma^{\prime}$ only. Particularly interesting two-particle correlators are the double occupancy $\left\langle n_{i \uparrow} n_{i \downarrow}\right\rangle$ and the spin-spin correlation $\left\langle\left(n_{i \uparrow}-n_{i \downarrow}\right)\left(n_{j \uparrow}-n_{j \downarrow}\right)\right\rangle$. Both will be important in the context of the simulations discussed in chapter 5 .

\subsection{Continuous-time auxiliary-field method}

The basis of the CT-AUX algorithm is the relation [148, 149]

$$
1-\frac{\beta U}{K}\left(n_{i \uparrow} n_{i \downarrow}-\frac{n_{i \uparrow}+n_{i \downarrow}}{2}\right)=\frac{1}{2} \sum_{s= \pm 1} \mathrm{e}^{\gamma s\left(n_{i \uparrow}-n_{i \downarrow}\right)}
$$

with

$$
\cosh (\gamma)=1+\frac{U \beta}{2 K}
$$

where $K$ is an arbitrary positive constant. The relation can be applied to the problem, if $H_{U}$ is modified to become

$$
\begin{aligned}
H_{U} & =U \sum_{i}\left(n_{i \uparrow} n_{i \downarrow}-\frac{n_{i \uparrow}+n_{i \downarrow}}{2}\right)-\frac{K}{\beta} \\
& =\frac{K}{2 \beta} \sum_{i} \sum_{s_{i}= \pm 1} \mathrm{e}^{\gamma s_{i}\left(n_{i \uparrow}-n_{i \downarrow}\right)}
\end{aligned}
$$

again causing only an energy shift. The transformation again introduces the Ising field $s_{i}$ into the algorithm. We expand the exponential in the partition function eq. (4.18) in the same fashion as in eq. (4.22). We treat the time ordering explicitly in the integration bounds and write

$$
\begin{aligned}
& Z=Z_{0} \sum_{k=0}^{\infty} \int_{0}^{\beta} \mathrm{d} \tau_{1} \cdots \int_{\tau_{k}-1}^{\beta} \mathrm{d} \tau_{k} \sum_{i_{1} s_{i_{1}}} \cdots \sum_{i_{k} s_{i}}\left(\frac{K}{2 \beta}\right)^{k} \times \\
& \operatorname{Tr} \prod_{m=k}^{1} \mathrm{e}^{-\Delta \tau_{m} H_{0}} \mathrm{e}^{\gamma s_{i_{m}}\left(n_{i_{m} \uparrow}\left(\tau_{m}\right)-n_{i_{m} \downarrow}\left(\tau_{m}\right)\right)}
\end{aligned}
$$

using $\Delta \tau_{m}=\tau_{m+1}-\tau_{m}$ for $m<k$ and $\Delta \tau_{k}=\beta-\tau_{k}+\tau_{1}$. This is very similar to equations in the Hirsch-Fye algorithm [75], e. g., see eq. (121) in ref. [48]. The trace over the string of exponential operators can be calculated explicitly,

$$
Z=Z_{0} \sum_{k=0}^{\infty} \int_{0}^{\beta} \mathrm{d} \tau_{1} \cdots \int_{\tau_{k}-1}^{\beta} \mathrm{d} \tau_{k} \sum_{i_{1} s_{i_{1}}} \cdots \sum_{i_{k} s_{i_{k}}}\left(\frac{K}{2 \beta}\right)^{k} \prod_{\sigma} N_{k \sigma}^{-1}
$$

using $G_{k \sigma}^{0}$ as defined in eq. (4.25) and

$$
N_{k \sigma}^{-1}=\operatorname{det}\left[\mathrm{e}^{V_{k \sigma}}-G_{k \sigma}^{0}\left(\mathrm{e}^{V_{k \sigma}}-1\right)\right]
$$


with

$$
\mathrm{e}^{V_{k \sigma}}=\operatorname{diag}\left(\mathrm{e}^{\gamma \operatorname{sgn}(\sigma) s_{i_{1}}}, \mathrm{e}^{\gamma \operatorname{sgn}(\sigma) s_{i_{2}}}, \ldots, \mathrm{e}^{\gamma \operatorname{sgn}(\sigma) s_{i_{k}}}\right) .
$$

Analogous to the CT-INT algorithm, $Z$ can be calculated as a sum over configurations $C$ with weights

$$
w(C)=Z_{0}\left(\frac{K}{2 \beta}\right)^{k} \prod_{\sigma} N_{k \sigma}^{-1} .
$$

\subsubsection{Monte Carlo updates}

The weights in eq. (4.50) possess the same structure as the weights of the CT-INT algorithm in eq. (4.29), if we identify $c=\frac{K}{2 \beta}$ and $M_{k \sigma}^{-1}=N_{k \sigma}^{-1}$. The acceptance probabilities eq. (4.33) remain therefore unchanged. One can employ additional spin-flip updates to speed up the performance of the algorithm. These updates flip randomly chosen Ising spins without altering the expansion order $k$. If two matrices $N_{k \sigma}$ and $N_{k \sigma}^{\prime}$ have the same imaginary time and space location for all vertices but differ in the value of one auxiliary spin, which is flipped from $s_{p}$ to $s_{p}^{\prime}$, their acceptance probability is given by

$$
a\left(C_{k} \rightarrow C_{k}^{\prime}\right)=\min \left(1, \prod_{\sigma} \frac{\operatorname{det} N_{k \sigma}}{\operatorname{det} N_{k \sigma}^{\prime}}\right) .
$$

Spin-flips are known from the Hirsch-Fye algorithm. Many aspects of this algorithm are still valid for the present continuous-time version. If we define the matrix $G_{k \sigma}$ analogous to eq. (4.25) using the full Green function $G_{i j}\left(\tau_{i}-\tau_{j}\right)$ instead of the effective bare Green function, $N_{k \sigma}$ is related to $G_{k \sigma}$ by $G_{k \sigma}=N_{k \sigma} G_{k \sigma}^{0}[75,48]$. Another crucial relation found by Hirsch and Fye is that the matrices $N_{k \sigma}$ and $N_{k \sigma}^{\prime}$ in eq. (4.51) are related by a Dyson equation

$$
\begin{aligned}
N_{i j}^{\prime} & =N_{i j}+\left(G_{i p}-\delta_{i p}\right) \lambda N_{p j}, \\
G_{i j}^{\prime} & =G_{i j}+\left(G_{i p}-\delta_{i p}\right) \lambda G_{p j}, \\
\lambda & =\mathrm{e}^{\gamma \operatorname{sgn}(\sigma)\left(s_{p}^{\prime}-s_{p}\right)}-1,
\end{aligned}
$$

omitting dependence on spin $\sigma$ and expansion order $k$. This follows directly from eq. (4.48). This relation allows for an efficient update of the configuration matrix $N_{k \sigma}$ and calculation of the acceptance probability. We use the "sub-matrix" update algorithm [56] described in appendix C, which performs a series of spin-flip and insertion/removal updates in a way that optimizes computer memory performance.

\subsubsection{Observables}

Note that the insertion of an arbitrary number of interaction vertices with "noninteracting spins" $s=0$ into eq. (4.46) does not change the value of the partition 
function. If we add two additional spins $s=s^{\prime}=0$ at sites $i, j$ and times $\tau, \tau^{\prime}$, we can derive an expression for the Green function measurement. $Z G_{\sigma i j}\left(\tau, \tau^{\prime}\right)$ is then given by an expression similar to eq. (4.46), with an insertion of $c_{i \sigma}(\tau)$ and $c_{j \sigma}^{\dagger}\left(\tau^{\prime}\right)$ at the corresponding times. Denoting with a tilde the corresponding enlarged matrices of size $k+2$, we can use the standard Hirsch-Fye formula (eq. (118) of ref. [48]) to obtain

$$
G_{\sigma i j}\left(\tau, \tau^{\prime}\right)=\frac{1}{Z} \sum_{C} w(C) \tilde{G}_{k \sigma} .
$$

Since $s=s^{\prime}=0$, the $\left(\tau, \tau^{\prime}\right)$ component of the matrix $\tilde{G}_{k \sigma}$ can be extracted from a simple block calculation,

$$
\begin{aligned}
G_{\sigma i j}\left(\tau-\tau^{\prime}\right) & =\mathcal{G}_{\sigma i j}\left(\tau-\tau^{\prime}\right) \\
& -\left\langle\sum_{p, q=1}^{k} \mathcal{G}_{\sigma i i_{p}}\left(\tau_{p}-\tau^{\prime}\right)\left[\left(\mathrm{e}^{V_{k \sigma}}-1\right) N_{k \sigma}\right]_{p q} \mathcal{G}_{\sigma i_{q} j}\left(\tau-\tau_{q}\right)\right\rangle_{w}
\end{aligned}
$$

This formula is identical to the measurement of the Green function in the CT-INT algorithm if we identify $M_{k \sigma}=\left(\mathrm{e}^{V_{k \sigma}}-1\right) N_{k \sigma}$. All other measurement formulas in section 4.2 .2 are therefore still valid.

\subsection{Summary}

We have given a description of the QMC algorithms that can be used to solve the quantum cluster problem of the DCA algorithm. In the next chapter, we show how the DCA in combination with these QMC methods can be applied to calculate thermodynamical properties of the Hubbard model.

Our implementation of the CT-INT and CT-AUX algorithms and the DCA framework described in the previous chapter is based on the libraries of the ALPS project $[3,9,57]$. ALPS (Applications and Libraries for Physics Simulations) is an open source effort providing libraries and simulation codes for strongly-correlated quantum-mechanical systems. The QMC algorithms were implemented in collaboration with Emanuel Gull. 


\section{Thermodynamics of the 3D Hubbard model on approach to the Néel transition}

In this chapter, we study the thermodynamic properties of the 3D Hubbard model for temperatures down to the Néel temperature $T_{\mathrm{N}}$. We perform large-scale DCA calculations using the methods outlined in the previous two chapters and controlled extrapolations to the infinite system size limit. We provide the full thermodynamical equation of state of the Hubbard model - in particular the entropy, energy, density, double occupancy, and nearest-neighbor spin-spin correlations - for interactions $U$ up to the bandwidth and for temperatures above $T_{\mathrm{N}}$.

We check the reliability of our approach by comparing our results with QMC simulations on finite lattices at half filling and high-temperature series expansion (HTSE) calculations.

To make contact with cold-gas experiments, we also compute properties of the system subject to an external trap in the local density approximation. We calculate the entropy required to reach the Néel state in an optical lattice experiment and provide the temperature dependence of observables that can be used for thermometry.

Major parts of this chapter were published in ref. [43]. HTSE calculations as well as all trap averages were calculated by Lode Pollet. Lattice QMC simulations were performed by Evgeny Kozik and Evgeny Burovski.

\subsection{Method}

We solve the Hubbard model within the DCA using the CT-AUX algorithm with sub-matrix updates introduced in section 4.3. We have performed extensive DCA calculations on bipartite clusters with $N_{\mathrm{c}}=18,26,36,48,56,64$. In order to achieve an optimal scaling behavior, we exclusively use the clusters determined in ref. [92] following the criteria in section 3.7. We show that an infinite system extrapolation can be done accurately and reliably in practice to obtain converged results for the Hubbard model directly in the thermodynamic limit [111, 92].

Fig. 5.1 shows, as a first example, the self-energy for the lowest Matsubara frequency $\mathrm{i} \omega_{0}$ along a path through the high-symmetry points of the Brillouin zone. The self-energies were calculated at the location of the discrete cluster momenta 

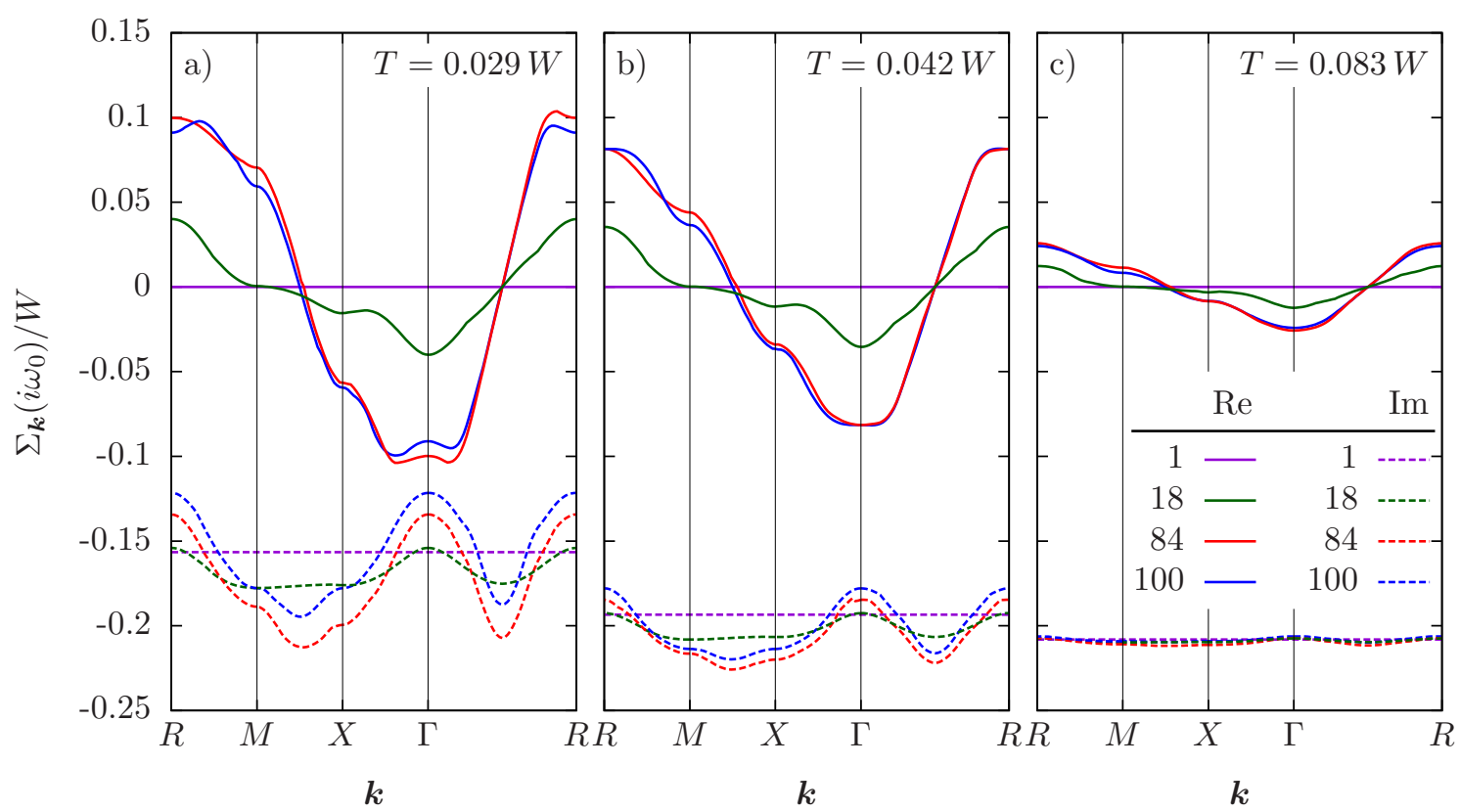

Figure 5.1: Real and imaginary parts of the lowest Matsubara frequency of the interpolated DCA self-energy $\Sigma_{\boldsymbol{k}}\left(\mathrm{i} \omega_{0}\right)$ for $U=0.67 \mathrm{~W}$ at half filling for three different temperatures. The lines denote DMFT results (horizontal straight lines) and results for clusters of size 18, 84, and 100. The self-energies are plotted along a path through the high-symmetry points of the first Brillouin zone (cf. fig. 7.1b for reference) and were interpolated using Akima splines [2].

$\boldsymbol{K}$ and were interpolated between these points using Akima splines in three dimensions. The high-temperature self-energy plotted in panel 5.1a is clearly converged as a function of cluster size, the intermediate temperature self-energy plotted in panel $5.1 \mathrm{~b}$ shows some cluster size dependence, and the right panel 5.1c shows a self-energy that even for 100 cluster sites is not yet converged - a sign of the long-ranged antiferromagnetic fluctuations important near $T_{\mathrm{N}}$. A reliable extrapolation of the cluster self-energy to the infinite-system limit would require even larger clusters.

While momentum-resolved self-energies show a very difficult convergence behavior, this is typically not true for momentum-averaged quantities. We measured energy, particle density, double-occupancy, and nearest-neighbor spin-spin correlation for each cluster size and extrapolate our results to the thermodynamic limit. While most observables can be measured directly along the lines of section 4.2.2, the evaluation of the kinetic energy demands more effort. We can express the kinetic energy using the single-particle Green function and the bare dispersion $\epsilon_{\boldsymbol{k}}$ via

$$
E_{\mathrm{kin}}=\sum_{\mathrm{i} \omega_{n}, \boldsymbol{k}} \epsilon_{\boldsymbol{k}} G_{\boldsymbol{k}}\left(\mathrm{i} \omega_{n}\right) \approx \frac{1}{V} \int \mathrm{d} \tilde{\boldsymbol{k}} \sum_{\mathrm{i} \omega_{n}, \boldsymbol{K}} \frac{\epsilon_{\boldsymbol{K}+\tilde{\boldsymbol{k}}}}{\mathrm{i} \omega_{n}+\mu-\epsilon_{\boldsymbol{K}+\tilde{\boldsymbol{k}}}-\Sigma_{\boldsymbol{K}}\left(\mathrm{i} \omega_{n}\right)} .
$$

The DCA enters here by approximating the self-energy part of the Green function 


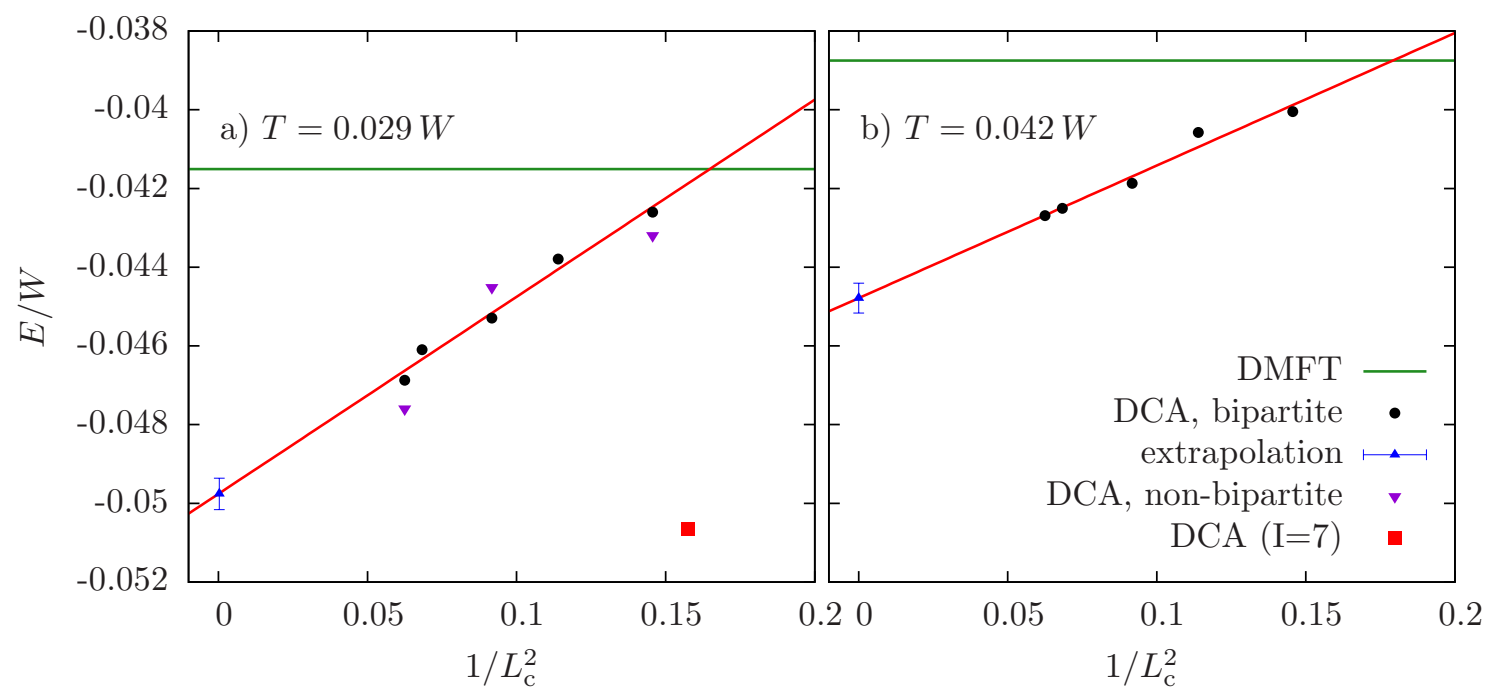

Figure 5.2: Finite-size scaling of the energy for $U=0.67 \mathrm{~W}$ at half filling for two different temperatures. DCA simulations on optimized bipartite clusters with $N_{\mathrm{c}}=18,26,36,56,64$ are taken into account. Monte Carlo errors are smaller than the symbol size. The DMFT energy is represented by a horizontal line. The left panel (a) presents additional DCA results for optimized non-biparte clusters with $N_{\mathrm{c}}=18,36,64$ and the result for the bipartite 16-site cluster described by $\boldsymbol{l}_{1}=(2,0,0), \boldsymbol{l}_{2}=(0,2,0), \boldsymbol{l}_{3}=(0,0,4)$ with - compared to the optimized clusters - high imperfection $I=7$ and cubicity $c=1.209$.

by the piecewise constant $\Sigma_{\boldsymbol{K}}\left(\mathrm{i} \omega_{n}\right)$.

Following the arguments in section 3.6, we assume $1 / L_{\mathrm{c}}^{2}$ finite-size scaling in the effective linear cluster size $L=N_{\mathrm{c}}^{1 / 3}$. Two scaling plots for $U=0.67 \mathrm{~W}$ at half filling are shown in fig. 5.2. The lower temperature in panel 5.2a corresponds approximately to $T_{\mathrm{N}}$, while the higher temperature in panel $5.2 \mathrm{~b}$ is far above $T_{\mathrm{N}}$. The figure shows the DMFT solution as a horizontal line and DCA estimates for five different bipartite clusters plotted against $1 / L_{\mathrm{c}}^{2}$. A linear least-squares fit to the DCA data produces a straight line whose interception with zero gives the extrapolated energy. The error due to the extrapolation is much larger compared to the statistical Monte Carlo errors. We therefore neglected the Monte Carlo errors in the process. Fig. 5.2a presents additional DCA results for optimized non-bipartite clusters. The nonbipartite clusters show a rather irregular scaling behavior compared to the bipartite ones. That indicates that the non-bipartite clusters may not only have a frustrating effect on antiferromagnetic order but on fluctuations as well. We therefore base our extrapolations exclusively on bipartite clusters. In order to demonstrate the relevance of topologically and geometrically optimized clusters, fig. 5.2a includes the energy of a 16-site cluster with high imperfection $I=7$ (red square), which is far off the scaling curve and thus unsuitable for finite-size scaling. 
In the immediate vicinity of the Néel temperature one would expect critical scaling behavior like $1 / L_{\mathrm{c}}^{1 / \nu}$. The critical exponent $\nu \approx 0.71$ [156] is known from the Heisenberg model whose universality class the Hubbard model belongs to. This ansatz has proven to be useful for the determination of the Néel temperature itself [92], but is not applicable to temperatures far above $T_{\mathrm{N}}$, which is the case for most of our data. Our scaling law should be replaced by critical scaling for data points which are very close to $T_{\mathrm{N}}$. However, such a procedure would introduce arbitrariness into the extrapolation, because the extent to which critical behavior is still valid away from $T_{\mathrm{N}}$ is unknown. We therefore consistently use the $1 / L_{\mathrm{c}}^{2}$ scaling and neglected critical finite-size scaling.

The entropy is not directly accessible to the QMC by the methods described in section 4.1 since it is not a thermal average but depends directly on the partition function $Z$. However, when the energy has been calculated on a sufficiently dense temperature grid, the entropy $S$ can subsequently be calculated by a numerical integration of the energy $E$ via

$$
\begin{aligned}
S(T) & =S\left(T_{\mathrm{u}}\right)-\int_{T}^{T_{\mathrm{u}}} \mathrm{d} T^{\prime} \frac{\mathrm{d} E}{T^{\prime} \mathrm{d} T^{\prime}} \\
& =S\left(T_{\mathrm{u}}\right)-\frac{E\left(T_{\mathrm{u}}\right)}{T_{\mathrm{u}}}+\frac{E(T)}{T}-\int_{T}^{T_{\mathrm{u}}} \mathrm{d} T^{\prime} \frac{E\left(T^{\prime}\right)}{T^{\prime 2}}
\end{aligned}
$$

up to a $T_{\mathrm{u}} \approx 3.5 \mathrm{~W}$, where the entropy $S\left(T_{u}\right)$ is accurately given by a hightemperature series expansion up to second order [15].

\subsection{Results at half filling}

We start our analysis at half filling, i. e., at chemical potential $\mu=U / 2$, and focus on $U=0.67 \mathrm{~W}$ where a comparison with results from lattice simulations $[144,29]$ is possible. We see in fig. 5.3 that the energy and entropy calculated using DCA and lattice QMC coincides within error bars at all temperatures. Agreement with a 10th order high temperature series expansion (HTSE) [161] is found down to $T \approx 0.1 \mathrm{~W}$. At that temperature single-site DMFT already deviates substantially, because this method misses short-range antiferromagnetic correlations. The Néel temperature was found to be $T_{\mathrm{N}} \approx 0.030(2) W$ in ref. [92]. The lattice QMC calculations find it at $T_{\mathrm{N}}=0.0278(6) W$. Using DCA, the critical entropy is $s:=S / N_{\mathrm{c}} \approx 0.46(4)$ for $T_{\mathrm{N}}$ according to ref. [92], and $s \approx 0.42(2)$ for $T_{\mathrm{N}}$ according to lattice QMC. In the rest of the chapter we will use $T_{\mathrm{N}}$ as determined by lattice QMC, where available, but entropies from DCA, since lattice QMC simulations are not possible away from half filling.

Temperatures $T \geq 0.03 W$ are reliably accessible for all but the largest interaction strength $U=W$, where we have been restricted to $T \geq 0.04 W$. The difference 

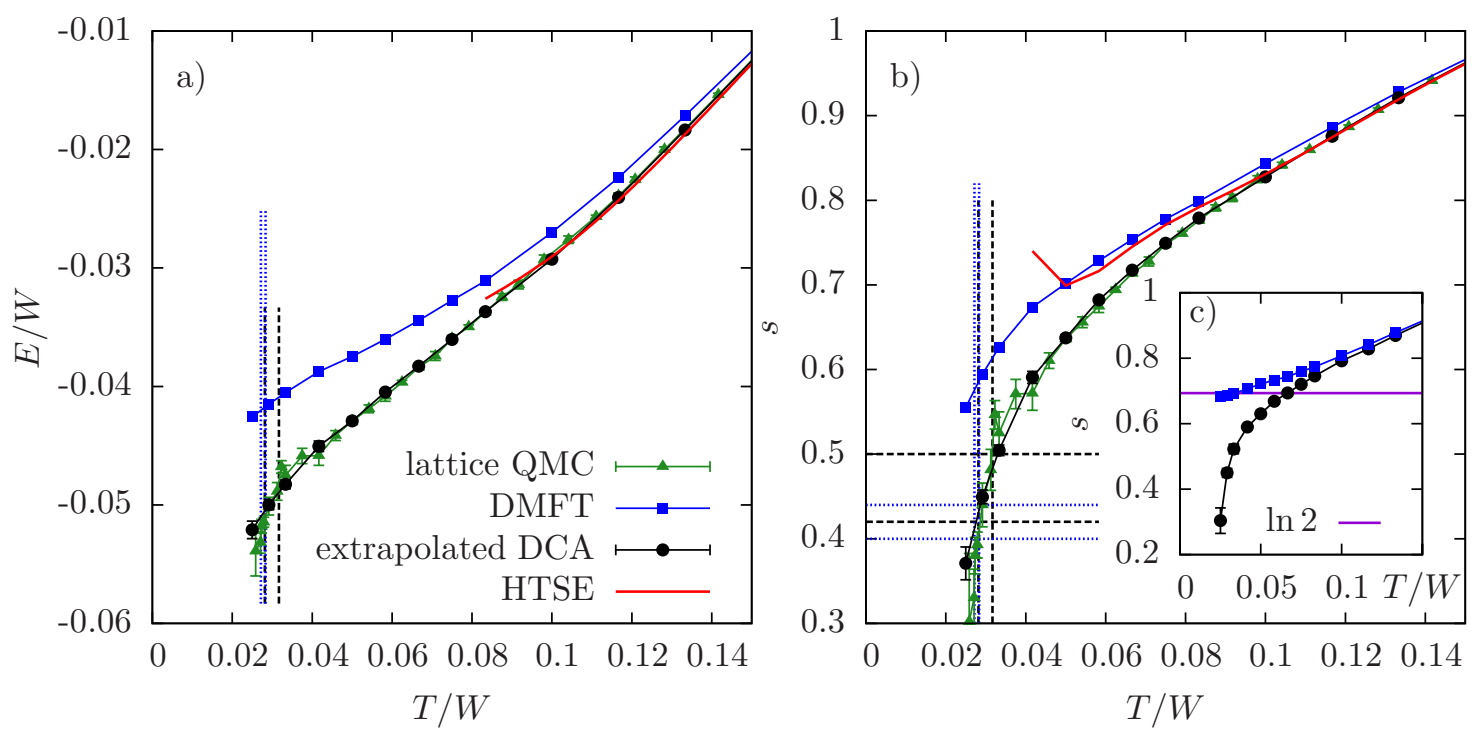

Figure 5.3: Energy per lattice site $E$ (a) and entropy per lattice site $s$ (b) as a function of temperature $T$, for $U=0.67 \mathrm{~W}$, at half filling. Dashed vertical lines (black): $T_{\mathrm{N}}$ from ref. [92], dotted lines (blue): according to lattice QMC. Dashed horizontal lines (black): entropy per lattice site $s$ at $T_{\mathrm{N}}$ [92], dotted lines (blue): according to lattice QMC. Lattice QMC data are extrapolated linearly in $1 / L_{\mathrm{c}}$ from simulation of cubic shaped lattice with $L_{\mathrm{c}}=6,8,10$. The inset (c) shows DMFT and extrapolated DCA entropies for $U=0.83 \mathrm{~W}$ at half filling.

between DMFT and DCA is even more pronounced for stronger interactions. For $U=0.83 W$ (fig. $5.3 \mathrm{c}$ ) the DMFT data for low temperatures are close to $\ln 2$, the entropy of a free spin. This indicates that the charge degrees of freedom are mostly frozen out, whereas the spin degrees of freedom are mostly unrestricted. Spin correlations, due to the antiferromagnetic fluctuations incorporated in the DCA, seem to cause a substantial decrease in entropy compared to DMFT.

The double occupancy, which has played a crucial role in optical lattice experiments $[88,163,161,173]$, is shown in fig. $5.4 \mathrm{a}$ as a function of temperature at half filling for different values of $U$. While for small $U$ a remarkable increase is seen on approach to $T_{\mathrm{N}}$, only a plateau remains at moderate values of $U$. This is in contrast to the DMFT predictions but similar to lattice QMC results in two dimensions [133]. For larger interactions $(U \gtrsim W)$, the double occupancy rises above that of a single site paramagnet, consistent with DMFT results for the antiferromagnetic phase below $T_{\mathrm{N}}$ [49]. The negative slope of the double occupancy, discussed in the context of single site DMFT [193], persists for a wide range of parameters. Sharp features just above $T_{\mathrm{N}}$, as detected in single site (momentum independent) studies [49], are not observed for the interaction values and temperature ranges studied here. Hence the 

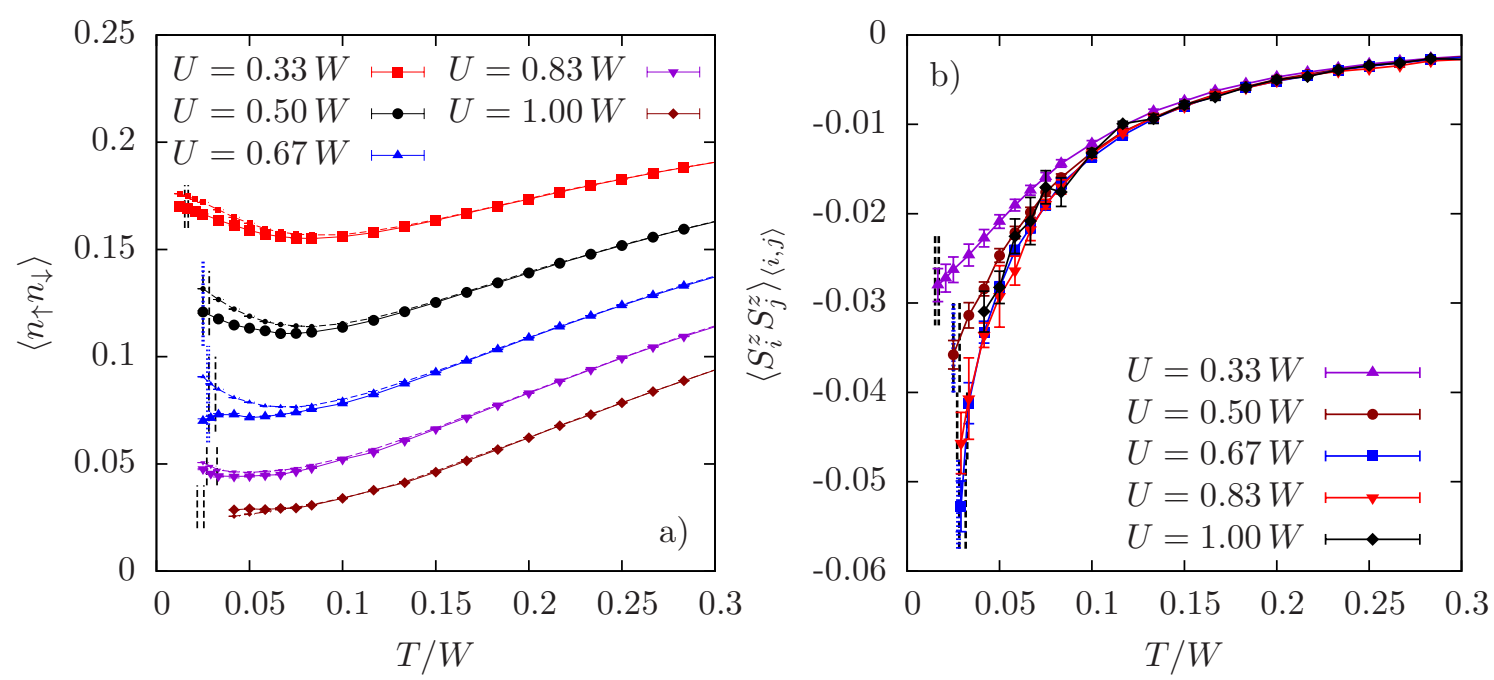

Figure 5.4: Double occupancy (a) and nearest-neighbor spin-spin correlation (b) as a function of $T$ at half filling. Extrapolated DCA results are shown as solid lines and DMFT values as dashed lines. Vertical lines: see fig. 5.3.

proposal that the double occupancy is a good candidate for thermometry $[89,49]$ is not substantiated by more accurate momentum-dependent calculations.

The spin-spin correlation function, plotted in fig. 5.4b as a function of temperature for various $U$, is only accessible in methods that include non-local correlations, but may be accessible experimentally [183]. It has a steep slope on approach to the Néel temperature, which makes it an ideal quantity for thermometry, in contrast to the double occupancy. This corresponds to the intuitive picture that charge degrees of freedom are already essentially frozen out around $T_{\mathrm{N}}$ while the spin degrees of freedom start to order there.

\subsection{Results away from half filling}

Away from half filling the sign problem hampers the performance of the QMC algorithm. Fig 5.5a shows the average sign close to $T_{\mathrm{N}}$ as a function of the filling $\langle n\rangle=$ $\left\langle n_{\uparrow}\right\rangle+\left\langle n_{\downarrow}\right\rangle$ for $U=0.67 W$ and different cluster sizes. The sign depends strongly on cluster size and its minimum as a function of filling is situated at $\langle n\rangle \approx 0.3$. This minimum corresponds to a chemical potential $\mu^{\prime}=\mu-U / 2=-0.58 \mathrm{~W}$ for the largest cluster with $N_{\mathrm{c}}=64$.

We study the temperature dependence of the sign for these particular parameters in fig. 5.5b. The figure exhibits a logarithmic plot of the sign as a function of the inverse temperature. The exponential nature of the sign problem is clearly visible in the linear slope at high $1 / T$. The sign of the converged DCA simulation is also compared to the sign of a finite-lattice calculation of the same cluster without the 

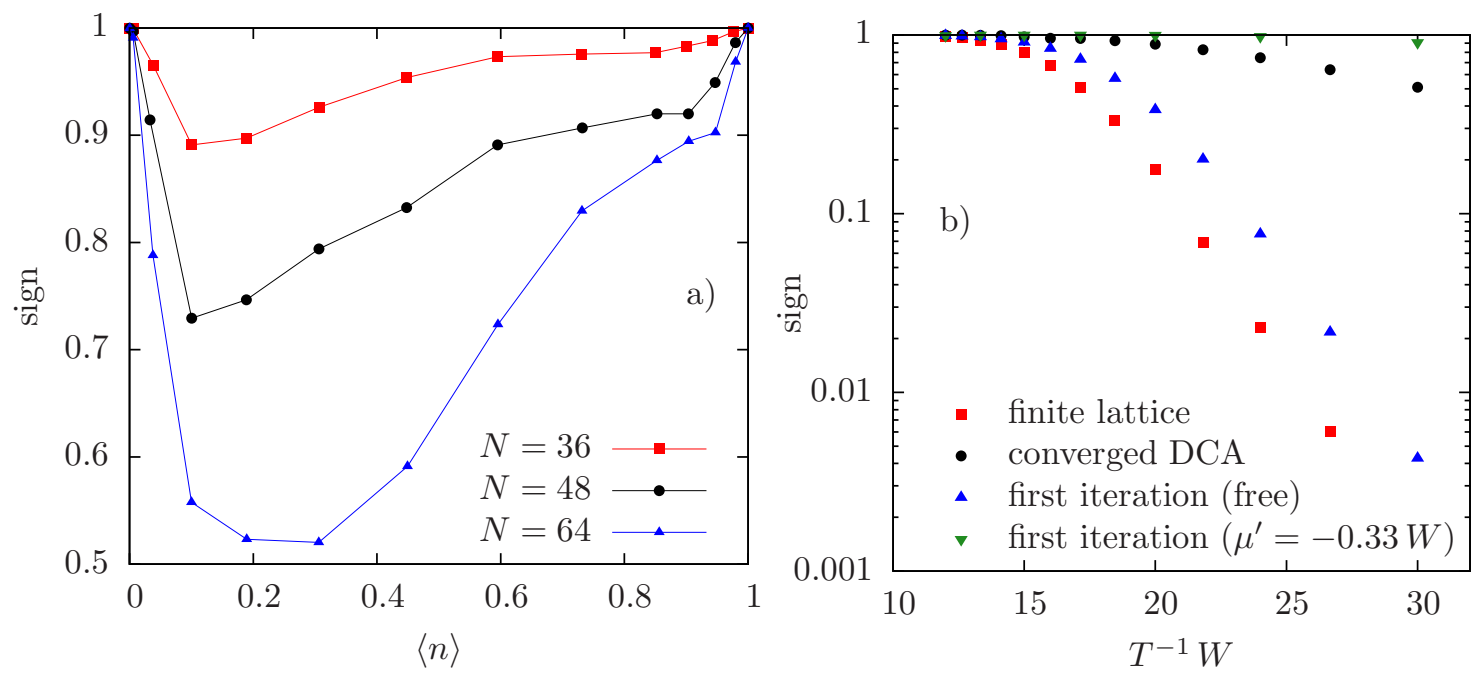

Figure 5.5: Average sign of the Monte Carlo simulation after convergence as a function of filling, for $U=0.67 \mathrm{~W}, T=0.025 \mathrm{~W}$, and for different cluster sizes (a). Panel b: Logarithmic sign for $N_{\mathrm{c}}=64$ at $\mu^{\prime}=\mu-U / 2=-0.58 \mathrm{~W}$ as function of $1 / T$. The figure depicts the sign for the converged DCA simulation as compared to a finite-lattice calculation, the sign of the first DCA iteration starting with the free solution, and the sign of the first DCA iteration starting with the converged result at $\mu^{\prime}=-0.33 \mathrm{~W}$.

DCA mean field. The comparison makes apparent that the average sign of the finite-lattice simulation is much smaller. For example, at $1 / T=30 \mathrm{~W}^{-1}$ the sign is already more than two orders of magnitude smaller than in the DCA calculation. This behavior effectively prohibits finite-lattice simulations away from half filling and illustrates how the DCA offers a possibility to study this parameter regime.

One should be aware of the fact, however, that the average sign is not the same for all DCA iterations. If we start the simulation with the usual free solution $\Sigma_{\boldsymbol{K}}\left(\mathrm{i} \omega_{n}\right)=$ 0 , the sign is almost as small as in the finite-lattice case (cf. fig. 5.5b). We can overcome this problem if we use the converged result of a simulation at the same temperature but at a slightly larger chemical potential $\mu^{\prime}=-0.33 \mathrm{~W}$ as starting point. In this case, the sign problem is even weaker than in the converged simulation. This illustrates that one should use a starting point which is as close as possible to the converged solution. We employ therefore the following strategy in our simulations: We perform simulations at fixed chemical potential $\mu$. The converged solution at a certain $\mu$ serves as the starting point of the simulation with next smaller chemical potential. If we start at half filling, where the sign problem is absent, we avoid bad starting configurations and can thereby iteratively simulate all chemical potentials.

After the discussion of the sign problem we proceed with simulation results. The suppression of entropy due to antiferromagnetic fluctuations in the DCA only takes 

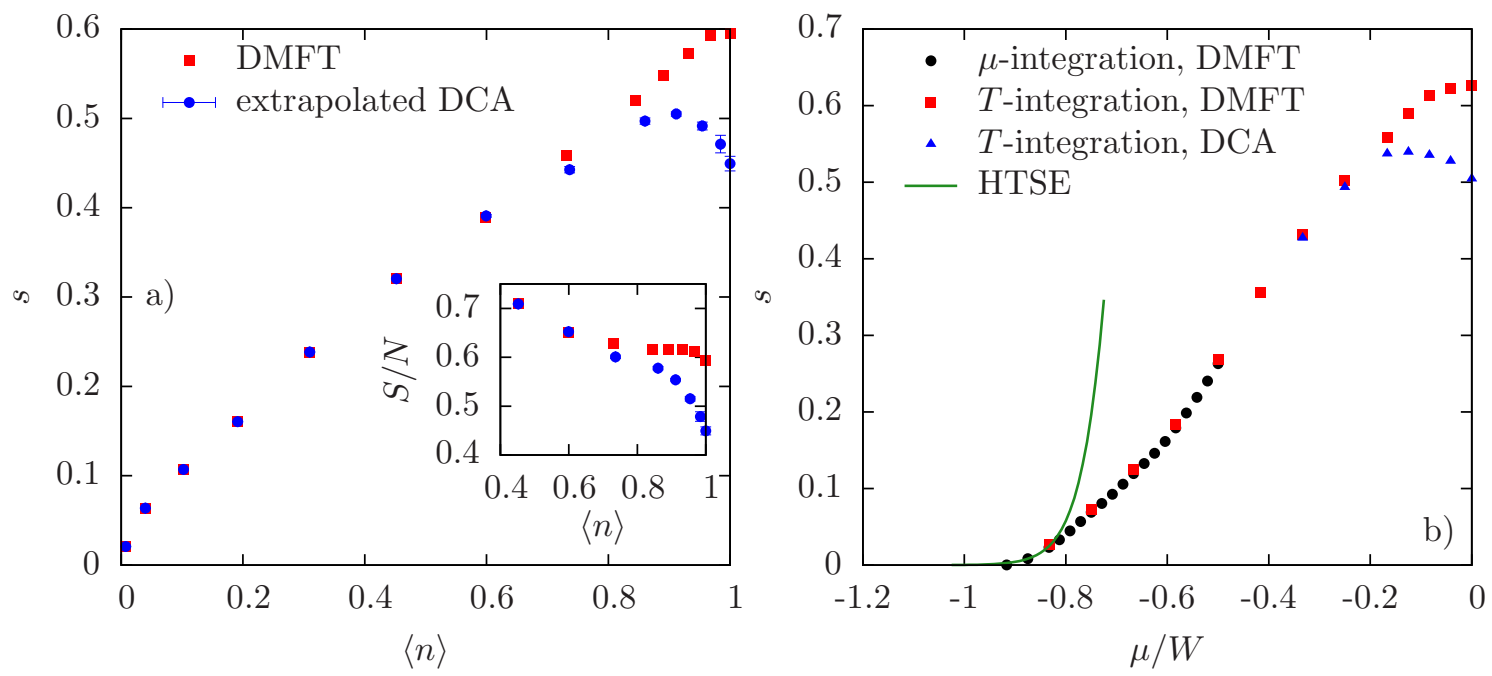

Figure 5.6: Panel a: Entropy per lattice site $s$ and the entropy per particle $S / N$ (inset) at $T=0.029 W \approx T_{\mathrm{N}}$ and $U=0.67 \mathrm{~W}$ as a function of $\langle n\rangle$. DMFT and extrapolated DCA values are shown. The errors are dominated by extrapolation errors. Panel b: Entropy for $T=0.033 \mathrm{~W}$ and $U=0.67 \mathrm{~W}$ as a function of the chemical potential $\mu$. Comparison of HTSE and different integration methods using either eq. (5.2) or eq. (5.3).

place near half filling. This can be seen in fig. 5.6, which shows the entropy per lattice site for $U=0.67 \mathrm{~W}$ near $T_{\mathrm{N}}$. The inset demonstrates that entropy per particle number $N$ increases strongly at lower densities. While single-site DMFT remains accurate for densities $n \lesssim 0.6$ due to the weak momentum dependence of the self-energy in this regime [54], the DCA results are important closer to half filling. Thus, DCA simulations are only necessary relatively close to half filling. We thereby avoid the region of minimal sign exhibited in fig. 5.5a.

The calculation of the entropy off half filling offers the possibility to test the accuracy of the integration formula eq. (5.2). The relation

$$
S(\mu, T)=\int_{-\infty}^{\mu} \mathrm{d} \mu^{\prime} \frac{\partial n\left(\mu^{\prime}, T\right)}{\partial T}
$$

i. e., integrating over chemical potential $\mu$, can serve as an alternative to the temperature integration eq. (5.2) [49] and yields the same results (fig. 5.6b). The figure also proves that, although the HTSE can be used to calculate the entropy, the approximation only holds for very low densities. We will use HTSE to calculate the low density part of a trap in the next section.

Similarly, near half filling, DMFT overestimates the double occupancy by $10 \%$ (fig. 5.7a), while deviations are less pronounced at lower densities. This observation 

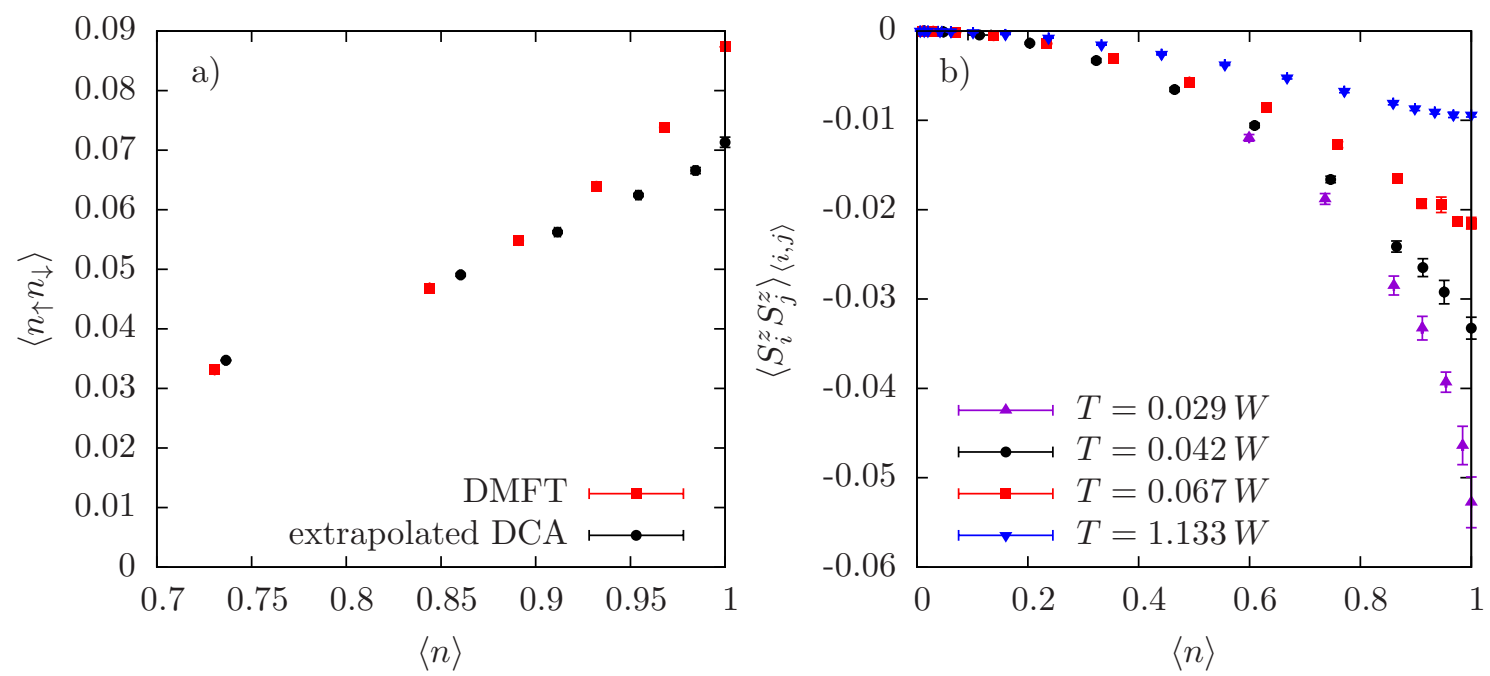

Figure 5.7: Double occupancy (a) and nearest-neighbor spin-spin correlation (b) for $U=$ $0.67 \mathrm{~W}$ and selected temperatures as a function of filling.

persists for all interactions and temperatures investigated. On the other hand, the spin-spin correlation function (fig. 5.7b) changes most rapidly near half filling when approaching $T_{\mathrm{N}}$ since it couples strongly to the developing (short-range) spin correlations.

\subsection{Entropy in the optical lattice system}

We now turn to the experimentally relevant case of an optical lattice in a harmonic trap, which is a closed system where entropy is conserved. So far, we have ignored the trap potential in all calculations. Now, we extend the Hubbard model by an energy potential $V_{i}$ on each lattice site $i$ (cf. eq. 2.17),

$$
H_{\text {trap }}=H+\sum_{\sigma i} V_{i} n_{i \sigma}
$$

We choose parameters close to current experiments: $V_{i}=0.004\left(\left|\boldsymbol{r}_{i}\right| / a\right)^{2} t$ with lattice spacing $a$, and we consider the case of half filling in the trap center $(\mu=U / 2)$. We treat the harmonic confinement in a local density approximation (LDA): for every site we perform a DCA simulation for a homogeneous system and average the results over the trap. LDA was found to be a good approximation for the Bose-Hubbard model for wide traps, except in close proximity to the critical point [197, 31, 32, 140] of the $\mathrm{U}(1)$ phase transition because of the diverging correlation length. In our setup LDA errors are small compared to errors from the uncertainty of $T_{\mathrm{N}}$.

Due to the large volume fraction, the wings of the gas may capture more entropy than the center of the trap, even though the entropy per site is comparable to the 

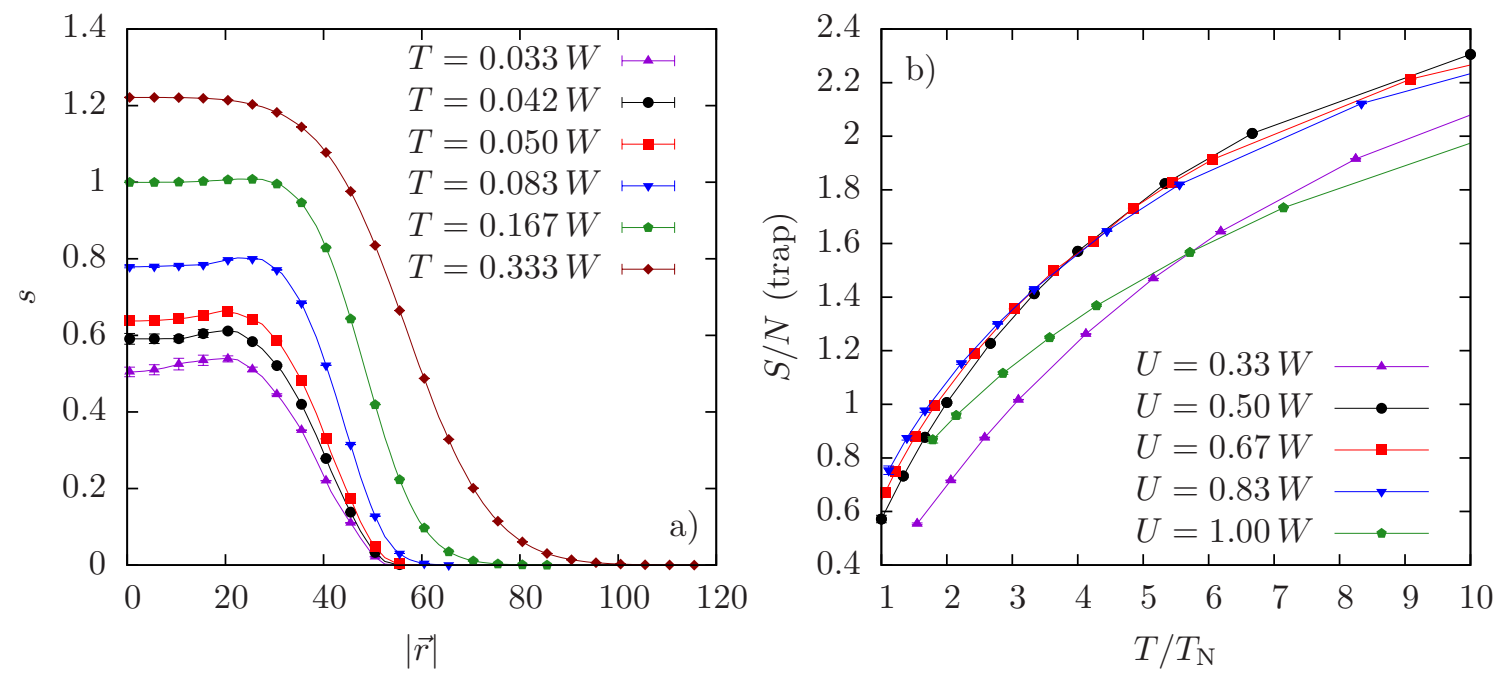

Figure 5.8: Panel a: Entropy profiles (entropy per lattice site) plotted over the trap in the LDA approximation for different temperatures with an interaction strength $U=0.67 \mathrm{~W}$. Error bars are shown every 5 lattice spacings, but are generally smaller than the symbol size. Panel b: Entropy per particle averaged over the trap as a function of temperature relative to $T_{\mathrm{N}}$ for different $U$. The Néel temperature is $T_{\mathrm{N}}=0.0278(6) \mathrm{W}$ for $U=0.67 \mathrm{~W}$ with an average entropy $S / N=0.65(6)$. Errors (not shown) in $S\left(T_{\mathrm{N}}\right)$ are estimated to be in the $10 \%$ range, with the largest contribution caused by the uncertainty in $T_{\mathrm{N}}$.

one in the center (see fig. 5.8a). In fact, the entropy of the whole density range $0.1<n<0.9$ is large. This opens the possibility to observe anti-ferromagnetic order in the trap center at an average entropy per particle over the trap which is about $50 \%$ larger than what could be expected from a homogeneous study. Optimal parameters are around $U=0.67 \mathrm{~W}$ when $T_{\mathrm{N}}=0.0278(6) W$ according to lattice QMC, corresponding to $S / N=0.65(6)$ in the trap, while $S / N=0.42(2)$ would be expected for a homogeneous system. As seen in fig. $5.8 \mathrm{~b}$, all $U$ in the range $0.67<U / W<1$ lead to similar conclusions. We have verified that changing the trap by a factor of 4 does not alter these conclusions.

\subsection{Summary}

We have provided the full thermodynamics of the 3D Hubbard model in the thermodynamic limit using the DCA formalism for $U \leq W$ and temperatures above the Néel temperature. Comparing to single-site DMFT, we found that the latter already fails at remarkably high temperatures $(T \approx 0.13 \mathrm{~W}$ for $U=0.67 \mathrm{~W}$ at the $1 \%$ level) near half filling. While the entropy per particle at the Néel temperature $T_{\mathrm{N}}=0.0278(6) W$ (determined with lattice QMC) is $S / N=0.42(2)$ for $U=0.67 \mathrm{~W}$ 
in a homogeneously half-filled system, we find that the Néel transition in a trap can already be reached at $S / N=0.65(6)$ in a realistically sized harmonic trap (taking $T_{\mathrm{N}}$ according to ref. [92] leads to $\left.S / N=0.69\right)$. Within the error of our calculation, taking any $U$ in the range $0.67<U / W<1$ is equally optimal for crossing the Néel temperature, and our conclusions do not change when the trap parameter is different.

We have also investigated the double occupancy and the nearest-neighbor spinspin correlation function as experimentally measurable quantities that were suggested to show precursors of antiferromagnetism. The double occupancy is almost flat as a function of temperature, while the spin correlations show a strong temperature dependence around the Néel temperature. This suggests that the spin correlations, not the double occupancy, are best suited to observe precursors of antiferromagnetism and measure the temperature. Our numerical data can be used to calibrate such a spin-correlation thermometer.

We have measured energy, entropy, density, double occupancy, and nearest-neighbor spin-spin correlations for many chemical potentials, temperatures above $T_{\mathrm{N}}$, and interaction strengths $U \leq W$. Since we could not present all results in this chapter, the complete data set can be obtained from tables in the supplementary material to ref. [43]. 


\section{Analytic continuation of quantum Monte Carlo data}

QMC algorithms provide powerful computational tools to calculate properties of interacting quantum many-particle systems. Of particular interest in those systems are dynamical correlation functions such as single-particle spectra or susceptibilities respectively dynamical structure factors. However, QMC presently provides data only on the imaginary time axis, and the necessary analytic continuation of these data has proven to be difficult.

The analytical structure of many-particle correlation functions is well known and, in principle, complete knowledge of their values along the imaginary time or frequency axis suffices to determine their values on the physically relevant real axis. If the correlation function is known analytically, this analytic continuation is indeed trivial in most cases. However, analytic continuation is one of many inverse problems in mathematics that are known to be ill-posed [7]. Although a unique solution of the problem exists, the solution is not stable with respect to even minimal variations in the input data. This means that - in the context of numerical methods, where only a finite number of discrete input data points can be calculated with often limited precision - a direct numerical solution of the inverse problem is impossible. The unavoidable statistical errors of Monte Carlo measurements further enhance the problem.

The standard tool to handle this difficulty is the maximum entropy method (MEM) [83]. It uses arguments of Bayesian logic [86, 61] to extract the most probable spectrum from the incomplete information provided by the data. The MEM is applied to a variety problems in data analysis in many fields of science and engineering [94]. Examples are image reconstruction [62], e. g., in astronomy [128], crystallography [155], spectroscopy of biological molecules [105], language processing [17], ecological modeling [139], or marketing research [65].

We give an overview over the maximum entropy techniques used to solve the analytic-continuation problem of QMC data. We especially focus on the Bayesian interpretation of the MEM. In order to solve the analytic-continuation problem efficiently, the MEM approximates all occurring probability distributions to be of Gaussian shape. In the past efforts were made to provide an alternative to this approach $[157,13,176]$. It was proposed to perform a Monte Carlo average over a wide range of spectra instead of selecting a single spectrum. So far, the method lacked a rigorous rule to eliminate a regularization parameter inherent in the algorithm. 
Although the MEM has been interpreted in terms of Bayesian inference [176], none of the authors utilized Bayesian logic to eliminate the above-mentioned regularization parameter. In this chapter, we derive a strict criterion to eliminate this free parameter, which is completely based on Bayesian logic. It uses Monte Carlo techniques to both calculate the average spectrum and to eliminate the regularization parameter. It treats all probabilities exactly and hereby avoids the approximations made in the standard maximum entropy method. The algorithm is applied to imaginary-frequency quantum Monte Carlo data. The resulting spectra are compared with results from standard maximum entropy calculations. Further applications of the MEM can be found in chapter 7, which deals with the calculation of momentum resolved single-particle spectra of the Hubbard model.

Major parts of this chapter were published in ref. [45]. A rudimentary implementation of the algorithm as initially described in ref. [13] and first tests on dynamical susceptibilities of one-dimensional quantum magnets were already presented in the author's diploma thesis [46].

\subsection{Analytic continuation}

For a finite temperature $T$, QMC simulations can provide accurate estimates $\bar{G}_{n}$ for either imaginary-time correlation functions $G(\tau)$ at a finite set of $N$ imaginary-time points $\tau_{n}$ or, alternatively, for imaginary-frequency correlation functions $G\left(\mathrm{i} \omega_{n}\right)$ at a finite set of $N$ Matsubara frequencies $\omega_{n}$ (cf. e. g., section 4.2.2 for the measurement of single-particle Green functions). The frequencies are defined as $\omega_{n}=(2 n+1) \pi / \beta$ for fermions and as $\omega_{n}=2 n \pi / \beta$ for bosons.

Because of the stochastic nature of Monte Carlo algorithms, each of the $\bar{G}_{n}$ possesses a known statistical error. Moreover, the data for the different time or frequency points are usually statistically highly correlated. Therefore, the input to the analytic-continuation procedure consists of the Monte Carlo estimates $\bar{G}_{i}$ and their covariance matrix

$$
C_{n m}=\overline{G_{n} G_{m}}-\bar{G}_{n} \bar{G}_{m} .
$$

In principle, the spectral function $A(\omega)=-\frac{1}{\pi} \operatorname{Im} G\left(\omega+i 0^{+}\right)$can be extracted from these data by inverting

$$
G_{n}=\int_{-\infty}^{\infty} \mathrm{d} \omega K_{n}(\omega) A(\omega)
$$

with

$$
K_{n}(\omega)=K\left(\tau_{n}, \omega\right):=-\frac{\mathrm{e}^{-\omega \tau_{n}}}{1 \pm \mathrm{e}^{-\omega \beta}}
$$

for time-dependent data or

$$
K_{n}(\omega)=K\left(\mathrm{i} \omega_{n}, \omega\right):= \pm \frac{1}{i \omega_{n}-\omega}
$$



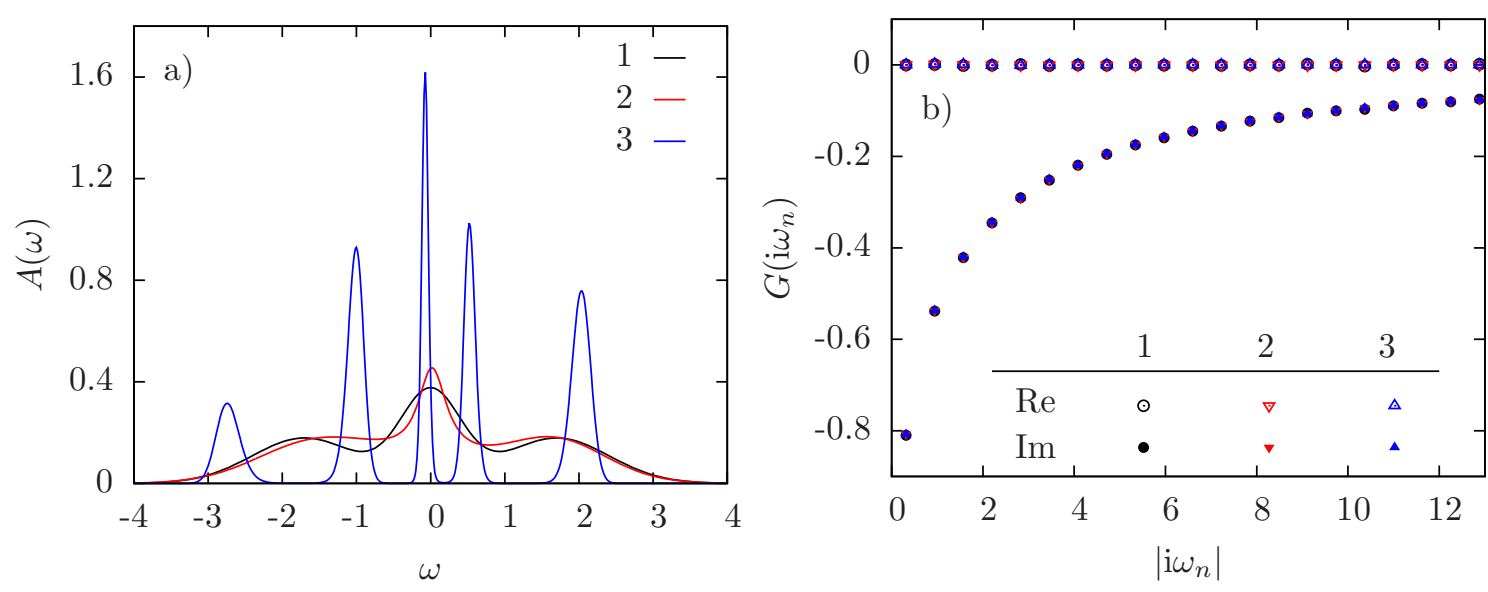

Figure 6.1: Three distinct spectra functions labeled by 1,2,3 (a) and their counterparts in imaginary frequencies (b) based on the fermionic kernel eq. (6.4) at $\beta=10$.

for frequency-dependent data, where the upper sign holds for fermions and the lower one for bosons.

Usually, one is interested in correlation functions of the form $G(\tau)=\left\langle T_{\tau} O(\tau) O^{\dagger}\right\rangle$, for example the local single-particle Green function $G_{\sigma i i}(\tau)=-\left\langle T_{\tau} c_{i \sigma}(\tau) c_{i \sigma}^{\dagger}\right\rangle$ (cf. eq. (3.5)). For fermionic operators $O$ and $O^{\dagger}$, the function $A(\omega)$ is real, non-negative for all $\omega$, and normalized to

$$
\mathcal{N}=\int_{-\infty}^{\infty} \mathrm{d} \omega A(\omega)
$$

For bosonic operators $O$ and $O^{\dagger}$, the same is true for the quantity $A(\omega) / \omega$. These properties are necessary for the analytic-continuation process. Both the MEM and the stochastic analytical inference discussed in section 6.3 rely on this behavior of $A(\omega)$. That means that, for example, a non-local single-particle Green function $G_{\sigma i j}(\tau)$ with $i \neq j$, where $A(\omega)$ has both positive and negative values, cannot be directly calculated by these methods. This has also consequences for the analytic continuation of antiferromagnetic self-energies described in section 7.3.

A direct inversion of eq. (6.2) is an ill-posed problem and numerically impossible. An alternative approach is a least-squares fit of $A(\omega)$ to the data $\bar{G}_{n}$, i. e., minimizing the $\chi^{2}$ estimate

$$
\chi^{2}[A]=\sum_{n, m}\left(\bar{G}_{n}-G_{n}\right)^{*} C_{n m}^{-1}\left(\bar{G}_{m}-G_{m}\right)
$$

with respect to $A(\omega)$. This approach, however, leads to a multitude of different solutions. Fig. 6.1 illustrates this effect. The apparently different spectra displayed in fig. 6.1a produce almost identical imaginary-frequency data (fig. 6.1b). The smallest 40 frequencies of the imaginary-frequency function of spectrum 1 in fig. 6.1a were 
used as a reference for a $\chi^{2}$ estimate after adding artificial Gaussian noise of width $\sigma=0.001$. With respect to these data, the $\chi^{2}$ estimate of the highly irregular spectrum 3 was even smaller than that of spectrum $2\left(\chi^{2}=27.5\right.$ compared to $\left.\chi^{2}=34.1\right)$, although spectrum 2 appears to be much closer to the input spectrum 1 .

This example illustrates, that a simple least-squares fit cannot solve the problem either. Additional assumptions about positivity, smoothness, or known moments of the spectrum can be applied to regularize the fit. Each of these assumptions involves a Lagrange parameter, which often cannot be determined unambiguously. Spectra calculated by these means are only qualitatively correct $[164,81]$.

\subsection{Maximum entropy method}

The maximum entropy method can be understood as a controlled attempt to regularize the least-squares fit described above. One defines the entropy

$$
S[A]=-\int_{-\infty}^{\infty} \mathrm{d} \omega A(\omega) \ln \frac{A(\omega)}{D(\omega)}
$$

relative to a default model $D(\omega)$. Any information, which is known about the spectrum beforehand, can be encoded in the default model. If $A(\omega)$ and $D(\omega)$ are non-negative and possess the same norm $\mathcal{N}$, the entropy $S$ will be non-positive and maximal for $D(\omega)$. Instead of just minimizing $\chi^{2}$ the MEM minimizes the quantity

$$
Q[A]=\frac{1}{2} \chi^{2}[A]-\alpha S[A]
$$

introducing a regularization parameter $\alpha>0$. This optimization problem can be numerically solved for fixed $\alpha$ to find the minimizing spectrum $\hat{A}_{\alpha}(\omega)$. In the limit of $\alpha \rightarrow \infty$ the spectrum minimizing $Q$ is the default model $D(\omega)$. For $\alpha \rightarrow 0$ the least-squares fit is regained. Thus, the parameter $\alpha$ interpolates between the least-squares fit and the default model.

There exist different approaches to find a criterion to eliminate the parameter $\alpha$. The simplest rule is to take the spectrum where $\chi^{2} \sim N$. This choice ensures that the differences between model and data are of the order of the error bars, thereby avoiding over-fitting. In order to derive more sophisticated methods, the MEM needs to be reinterpreted by means of Bayesian statistical inference [86, 61].

\subsubsection{Bayesian statistical inference}

The MEM can be reformulated by defining probabilities for the quantities involved in the analytic-continuation problem. Let $P[A]$ denote the prior probability of the spectrum $A(\omega) . P[A \mid \bar{G}]$ denotes the posterior probability of $A$ given the input data $\bar{G}$ 
and $P[\bar{G} \mid A]$ the likelihood function. Bayes's Theorem $[134]$ relates these probabilities to each other,

$$
P[A \mid \bar{G}]=P[\bar{G} \mid A] P[A] / P[\bar{G}] .
$$

The probability $P[\bar{G}]$ is called the evidence and serves as normalization for the posterior probability $P[A \mid \bar{G}]$,

$$
P[\bar{G}]=\int \mathcal{D} A P[\bar{G} \mid A] P[A] .
$$

One identifies

$$
P[\bar{G} \mid A]=\frac{1}{Z_{1}} \exp \left(-\frac{1}{2} \chi^{2}[A]\right)
$$

and

$$
P[A]=\frac{1}{Z_{2}} \exp (\alpha S[A])
$$

The quantities

$$
Z_{1}=\int \mathcal{D} \bar{G} \mathrm{e}^{-\frac{1}{2} \chi^{2}[A]}
$$

and

$$
Z_{2}=\int \mathcal{D} A \mathrm{e}^{\alpha S[A]}
$$

normalize the respective probabilities. This way, the posterior probability can be rewritten as

$$
P[A \mid \bar{G}]=\frac{\mathrm{e}^{-Q[A]}}{Z_{1} Z_{2} P[\bar{G}]}
$$

with

$$
P[\bar{G}]=\frac{\int \mathcal{D} A \mathrm{e}^{-Q[A]}}{Z_{1} Z_{2}} .
$$

Thus, the minimization of $Q$ can be reinterpreted as the maximization of the posterior probability $P[A \mid \bar{G}] \propto \mathrm{e}^{-Q}$. The MEM therefore determines the most probable spectrum $\hat{A}_{\alpha}$ given the input data $\bar{G}$.

\subsubsection{Bayesian inference and the regularization parameter $\alpha$}

This alternative formulation of the problem provides the necessary tools to eliminate the free parameter $\alpha[168,169]$. Eq. (6.9) can be rewritten including $\alpha$ :

$$
P[A, \alpha \mid \bar{G}]=P[\bar{G} \mid A, \alpha] P[A, \alpha] / P[\bar{G}] .
$$

If one applies Bayes's theorem to factorize $P[A, \alpha]$ and integrates over $A$, the relation

$$
\begin{aligned}
P[\alpha \mid \bar{G}] & =P[\alpha] \int \mathcal{D} A P[\bar{G} \mid A, \alpha] P[A \mid \alpha] / P[\bar{G}] \\
& =\frac{P[\alpha]}{Z_{1} Z_{2} P[\bar{G}]} \int \mathcal{D} A \mathrm{e}^{-Q[A]}
\end{aligned}
$$


for the posterior probability $P[\alpha \mid \bar{G}]$ can be found. Analogous to the argument given above, one identifies $P[\bar{G} \mid A, \alpha] \propto \exp \left(-\frac{1}{2} \chi^{2}[A]\right)$ and $P[A \mid \alpha] \propto \exp (\alpha S[A])$. The evidence

$$
P[\bar{G}]=\int_{0}^{\infty} \mathrm{d} \alpha \frac{P[\alpha] \int \mathcal{D} A \mathrm{e}^{-Q[A]}}{Z_{1} Z_{2}}
$$

is an $\alpha$-independent normalization constant. All quantities in this equation are known except $P[\alpha]$, the prior probability of $\alpha$. It is either taken to be constant or to be the Jeffreys prior $1 / \alpha[53,169,25]$. However, the choice of $P[\alpha]$ turns out to be of little influence on the resulting spectra.

By assuming all probabilities involved to be of Gaussian shape, a treatment of eqs. (6.15) and (6.18) by a numerical optimization process becomes possible. Details of the algorithm are described in appendix D. There are still two alternatives to treat the parameter $\alpha$ :

1. one calculates $\alpha^{*}$ as the $\alpha$ that maximizes $P[\alpha \mid \bar{G}]$ and takes $\hat{A}_{\alpha^{*}}$ as the final result for the spectral function $[168,169]$;

2. one averages over all $\hat{A}_{\alpha}$ weighted by the posterior probability of $\alpha$, i.e., the average spectrum

$$
\langle A\rangle=\int_{0}^{\infty} \mathrm{d} \alpha P[\alpha \mid \bar{G}] \hat{A}_{\alpha}
$$

is taken as the final result [25].

It is a priori not clear, which of the two algorithms is favorable.

\subsection{Stochastic analytical inference}

Stochastic analytical inference (SAI) is an alternative to the standard MEM which does not employ the explicit regularization of the fit by the entropy eq. (6.7). Rather than maximizing $P[A \mid \bar{G}]$, an average over all possible spectra weighted by

$$
w \propto \exp \left(-\frac{1}{2} \chi^{2} / \alpha\right)
$$

is performed. BEACH refined this approach, by introducing the default model $D(\omega)$ of the MEM into the algorithm [13]. By mapping $\omega$ on $x \in[0,1]$ using

$$
x=\phi(\omega)=\frac{1}{\mathcal{N}} \int_{-\infty}^{\omega} d \omega^{\prime} D\left(\omega^{\prime}\right)
$$


a dimensionless field $n(x)$ can be defined as

$$
n(x)=\frac{A\left(\phi^{-1}(x)\right)}{D\left(\phi^{-1}(x)\right)} .
$$

The field $n(x)$ is normalized to 1 ,

$$
1=\int_{0}^{1} \mathrm{~d} x n(x) .
$$

The correlation function eq. (6.2) is rewritten as

$$
G_{n}=\int_{0}^{1} \mathrm{~d} x \hat{K}_{n}(x) n(x)
$$

using $\mathcal{N} \hat{K}_{n}(\phi(\omega))=K_{n}(\omega)$. One calculates the average field

$$
\langle n(x)\rangle_{\alpha}=\frac{1}{Z} \int \mathcal{D}^{\prime} n(x) n(x) \mathrm{e}^{-\frac{1}{2} \chi^{2}[n(x)] / \alpha}
$$

with

$$
Z=\int \mathcal{D}^{\prime} n(x) \mathrm{e}^{-\frac{1}{2} \chi^{2}[n(x)] / \alpha}
$$

The measure

$$
\mathcal{D}^{\prime} n(x)=\mathcal{D} n(x) \Theta[n] \delta\left(\int_{0}^{1} \mathrm{~d} x n(x)-1\right)
$$

restricts the integration to fields $n(x)$ that satisfy norm rule eq. (6.24) and the positivity requirement. In eq. (6.28),

$$
\Theta[n]=\left\{\begin{array}{l}
1 \text { if } \forall x: n(x) \geq 0 \\
0 \text { otherwise }
\end{array}\right.
$$

The average spectrum $\langle A\rangle_{\alpha}$ can then be regained via

$$
\langle A(\omega)\rangle_{\alpha}=D(\omega)\langle n(\phi(\omega))\rangle_{\alpha}
$$

If $\frac{1}{2} \chi^{2}$ is interpreted as the Hamiltonian of a fictitious physical system, eq. (6.26) possesses the structure of a canonical ensemble average at a temperature $\alpha$. The laws of statistical mechanics then state that the average spectral function $\langle A\rangle_{\alpha}$ minimizes the free energy

$$
F=\frac{1}{2}\left\langle\chi^{2}\right\rangle_{\alpha}-\alpha \mathcal{S}
$$

This expression displays a similar structure as eq. (6.8). Thus, the averaging process implicitly generates an entropy $\mathcal{S}$. However, this entropy does not have the explicit 
form of eq. (6.7). In the limit $\alpha \rightarrow 0$ the averaging process minimizes $\chi^{2}$. Whereas in the limit $\alpha \rightarrow \infty$ the average in eq. (6.26) is completely unaffected by $\chi^{2}$ and will constrained by eq. (6.24) - result in $\langle n(x)\rangle=1$. In this case, the resulting spectrum is the default model. The algorithm therefore exhibits the same limiting cases as the MEM. BEACH further showed that a mean-field treatment of the fictitious physical system described by $\frac{1}{2} \chi^{2}[n(x)]$ is formally equivalent to the MEM [13]. Thus, at least in this respect, the SAI represents a generalization of the MEM.

\subsubsection{Bayesian statistical inference}

The remaining open question, namely, how to eliminate the parameter $\alpha$, was addressed by all preceding authors differently:

1. SANDVIK proposed to examine the plot of the average entropy against $\alpha$ and identifies the final $\alpha$ by a sharp drop in the entropy curve [157];

2. BEACH examined a double-logarithmic plot of the average $\chi^{2}$ and identifies the final $\alpha$ by a kink in the $\chi^{2}$ curve [13];

3. SyluUÅsen argues to take $\alpha=1$ [176].

Especially the first two criteria are merely based on heuristic arguments. The simple rule to take $\chi^{2} \sim N$ is also applicable to this method and should be mentioned here.

In the following, we will use Bayesian inference to derive a criterion to eliminate the regularization parameter $\alpha$. In contrast to the MEM, the stochastic analytical inference does not maximize the posterior probability $P[A \mid \bar{G}]$. Instead, it averages all possible fields $n$ (omitting the argument $x$ in the progress) weighted by $P[n \mid \bar{G}]$,

$$
\langle n\rangle=\int \mathcal{D} n n P[n \mid \bar{G}]
$$

Bayes's theorem can be applied to factorize $P[n \mid \bar{G}]$ analogous to eq. (6.9),

$$
P[n \mid \bar{G}]=P[\bar{G} \mid n] P[n] / P[\bar{G}] .
$$

The SAI does not introduce an explicit entropy term. Following ref. [176] only the positivity requirement and norm rule eq. (6.24) enter the prior probability

$$
P[n]=\Theta[n] \delta\left(\int_{0}^{1} \mathrm{~d} x n(x)-1\right) .
$$

The likelihood function is identified as

$$
P[\bar{G} \mid n]=\frac{1}{Z^{\prime}} \mathrm{e}^{-\frac{1}{2} \chi^{2} / \alpha} .
$$


By evaluating the Gaussian integral, the normalization $Z^{\prime}$ is readily calculated to be

$$
Z^{\prime}=\int \mathcal{D} \bar{G} \mathrm{e}^{-\frac{1}{2} \chi^{2} / \alpha}=(2 \pi \alpha)^{N / 2} \sqrt{\operatorname{det} C}
$$

Using

$$
P[\bar{G}]=\int \mathcal{D}^{\prime} n \frac{\mathrm{e}^{-\frac{1}{2} \chi^{2}[n] / \alpha}}{Z^{\prime}}=\frac{Z}{Z^{\prime}}
$$

the posterior probability results in

$$
P[n \mid \bar{G}]=\Theta[n] \delta\left(\int_{0}^{1} \mathrm{~d} x n(x)-1\right) \frac{1}{Z} \mathrm{e}^{-\frac{1}{2} \chi^{2}[n] / \alpha},
$$

as expected from the comparison of eqs. (6.26) and (6.32).

\subsubsection{Bayesian inference and the regularization parameter $\alpha$}

Bayesian logic can also be utilized to calculate the posterior probability $P[\alpha \mid \bar{G}]$. Substituting $n$ for $A$ in eq. (6.18) and identifying $P[n \mid \alpha]=P[n]$ with $P[n]$ from eq. (6.34) and correspondingly $P[\bar{G} \mid n, \alpha]=P[\bar{G} \mid n]$ with $P[\bar{G} \mid n]$ from eq. (6.35), one obtains

$$
\begin{aligned}
P[\alpha \mid \bar{G}] & =P[\alpha] \int \mathcal{D} n P[\bar{G} \mid n, \alpha] P[n \mid \alpha] / P[\bar{G}] \\
& =\frac{P[\alpha]}{Z^{\prime} P[\bar{G}]} \int \mathcal{D}^{\prime} n \mathrm{e}^{-\frac{1}{2} \chi^{2}[n] / \alpha} .
\end{aligned}
$$

The evidence

$$
P[\bar{G}]=\int_{0}^{\infty} \mathrm{d} \alpha \frac{P[\alpha] \mathrm{e}^{-\frac{1}{2} \chi^{2} / \alpha}}{Z^{\prime}(\alpha)}
$$

is again an $\alpha$-independent normalization constant. The combination of eqs. (6.36) and (6.39) gives the final expression for the $\alpha$-dependence of the posterior probability,

$$
P[\alpha \mid \bar{G}] \propto P[\alpha] \alpha^{-N / 2} \int \mathcal{D} n \mathrm{e}^{-\frac{1}{2} \chi^{2}[n] / \alpha} .
$$

Analogous to the MEM one has two possibilities to treat the regularization parameter:

1. one calculates $\alpha^{*}$ as the $\alpha$ that maximizes $P[\alpha \mid \bar{G}]$ and takes $\langle n\rangle_{\alpha^{*}}$ as the final result; 
2. one averages over all $\langle n\rangle_{\alpha}$ weighted by the posterior probability of $\alpha$, i.e., the average field

$$
\left\langle\langle n\rangle_{\alpha}\right\rangle=\int_{0}^{\infty} \mathrm{d} \alpha P[\alpha \mid \bar{G}]\langle n\rangle_{\alpha}
$$

is taken as the final result.

\subsection{Monte Carlo evaluation}

In order to calculate the quantities appearing in eqs. (6.26) and (6.41), a numerically treatable approximation for the field configuration $n(x)$ and the integration measure $\mathcal{D}^{\prime} n$ has to be found. The average eq. (6.26) is evaluated by a standard Monte Carlo simulation, treating the regularization parameter $\alpha$ as the temperature of the system. The simulation has to be performed for a wide range of different $\alpha$-values, which makes a parallel-tempering algorithm $[175,108,112]$ necessary to ensure convergence for small $\alpha$. In order to measure the average field configuration, a histogram of the delta function walkers is recorded. Our implementation closely follows ref. [13] and is further detailed in appendix D.

A particular problem in the proposed approach is that a numerical treatment of eq. (6.41) involves the calculation of the quantity

$$
Z=\int \mathcal{D}^{\prime} n \mathrm{e}^{-\frac{1}{2} \chi^{2} / \alpha}
$$

This is equivalent to calculating a partition function in a canonical ensemble at temperature $\alpha$. Standard Monte Carlo techniques are only able to calculate thermal expectation values but not the partition function itself. We therefore use a WangLandau algorithm $[189,188]$ to generate the density of states $\rho(E)$ of the system. Once $\rho(E)$ is calculated, the partition function can be obtained by

$$
Z=\int \mathrm{d} E \rho(E) \mathrm{e}^{-E / \alpha} .
$$

The Wang-Landau algorithm was developed to overcome critical slowing down of Monte Carlo simulations near a first-order phase transition. It is generally useful for systems with complex free-energy landscapes. Here, we only utilize its ability to calculate the partition function directly.

\subsection{Simulation results}

We apply the algorithm to imaginary-time data from quantum Monte Carlo simulations. As test case we consider the single-band Hubbard model eq. (2.1) in two 


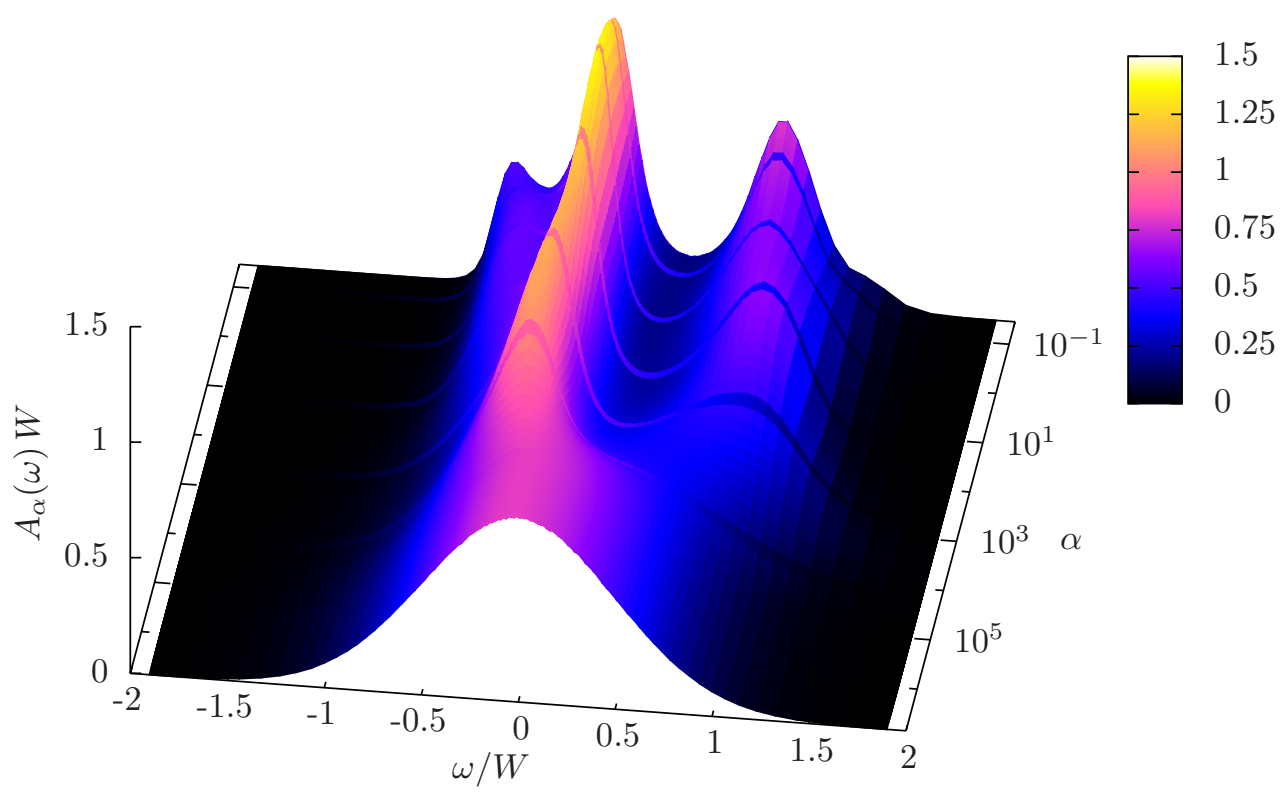

Figure 6.2: Simulated spectra for a range of regularization parameters $\alpha\left(\beta=14 W^{-1}\right)$. For large $\alpha$ the Gaussian shape of the default model is visible. For decreasing $\alpha$ several features begin to appear.

dimensions. The full lattice model was approximated by a square-shaped $2 \times 2$ cluster embedded in a mean field using the DCA. The single-particle Green function was calculated by QMC using the CT-INT algorithm (see section 4.2). The model was simulated for $U=W$, where $W=8 t$ denotes the bandwidth, and at fixed filling $\langle n\rangle=\left\langle n_{\uparrow}+n_{\downarrow}\right\rangle=0.9$ for several temperatures $T$. Since it is possible to calculate the Green function directly in frequency space (eq. (4.39)), no Fourier transformation or discretization of the imaginary time axis is necessary. In all simulations the number of measured Matsubara frequencies was restricted to $n_{\max }=2 U \beta$, which has proven to be sufficient for all calculation. A further increase in the number of frequencies had no influence on the analytic-continuation results.

Figure 6.2 shows the $\alpha$ dependence of the single-particle spectra calculated by the parallel-tempering Monte Carlo simulation $\left(\beta=14 W^{-1}\right)$. A Gaussian default model

$$
D(\omega)=\frac{1}{\sqrt{2 \pi \sigma}} \mathrm{e}^{-\omega^{2} / 2 \sigma}
$$

with $\sigma=0.25 W^{2}$ was used. The shape of the default model is clearly visible for large $\alpha$. One can see how several different peaks and other structures appear for decreasing $\alpha$. Since the $\alpha$ dependence is so strong, one definitely needs a criterion to eliminate the regularization parameter.

The density of states calculated by the Wang-Landau simulation and the probabil- 

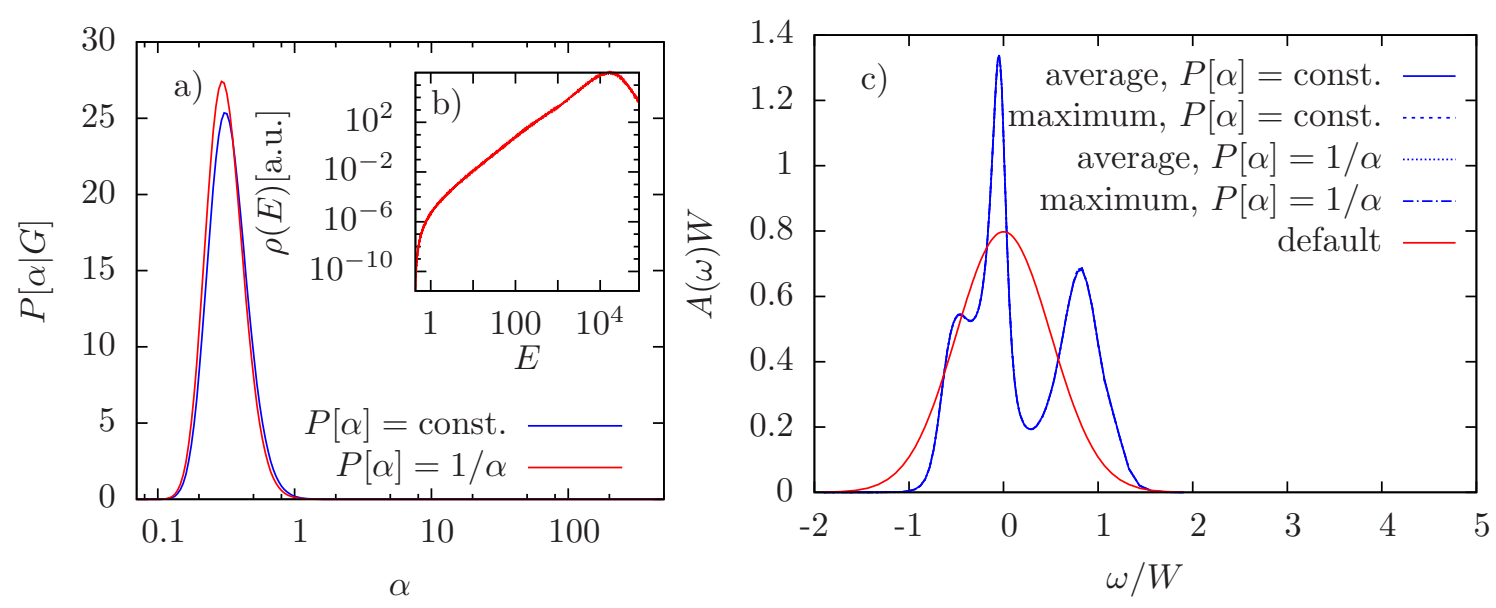

Figure 6.3: The probability distributions $P[\alpha \mid \bar{G}]$ (a) based on a Wang-Landau simulation of the density of states (b). The different choices for $P[\alpha]$ only have a weak influence on the position of the peak. The resulting spectra (c) are calculated by either averaging all spectra over $P[\alpha \mid \bar{G}]$ or by taking the spectrum that maximizes them. The four different spectra are practically identical.

ity distribution $P[\alpha \mid \bar{G}]$ following eq. (6.41) is shown in fig. 6.3. $P[\alpha \mid \bar{G}]$ is plotted for the two most common choices for $P[\alpha]$, i. e., $P[\alpha]=$ constant and $P[\alpha]=1 / \alpha$. The density of states varies over at least 15 orders of magnitude (note the logarithmic scales). The probability distributions $P[\alpha \mid \bar{G}]$ exhibit a well-defined peak at $\hat{\alpha} \approx 0.2$. Note that the two different choices for $P[\alpha]$ have only weak influence on the position of the peak. The two different probability distributions are used to calculate the final single-particle spectrum. Following the discussion in section 6.3.2, fig. 6.3 shows the average of all spectra of fig. 6.2 weighted by $P[\alpha \mid \bar{G}]$ and the spectrum whose $\alpha$ maximizes $P[\alpha \mid \bar{G}]$. The resulting spectra are nearly indistinguishable and show that neither the ambiguity in the treatment of the probability distribution nor the choice of $P[\alpha]$ has a significant influence on the resulting spectrum.

We compare our results from the stochastic analytical inference with those obtained with other methods to fix $\alpha$. Fig. 6.4a shows that the point where $\chi^{2} \sim N$ corresponds to $\alpha \approx 1$. That means that the $\alpha$ determined by this rule is identical to Syljuåsen's choice. The $\chi^{2}$ estimate also exhibits a kink in the very same $\alpha$ region. Thus, the spectra determined by all three methods are identical (fig. 6.4c). The chosen $\alpha=1$ is larger than $\hat{\alpha} \approx 0.2$. That indicates that the spectra determined with this criterion are stronger regularized than the spectra calculated by the probability distributions in fig. 6.3. However, at least for the QMC data under consideration, the difference between the two spectra is only small. The entropy (fig. 6.4b) shows no significant features and gives no indication how to choose the parameter $\alpha$. A sharp drop in the entropy curve is not visible in the simulated area.

Finally, we compare the SAI with the standard MEM approach. Figure 6.5 shows 

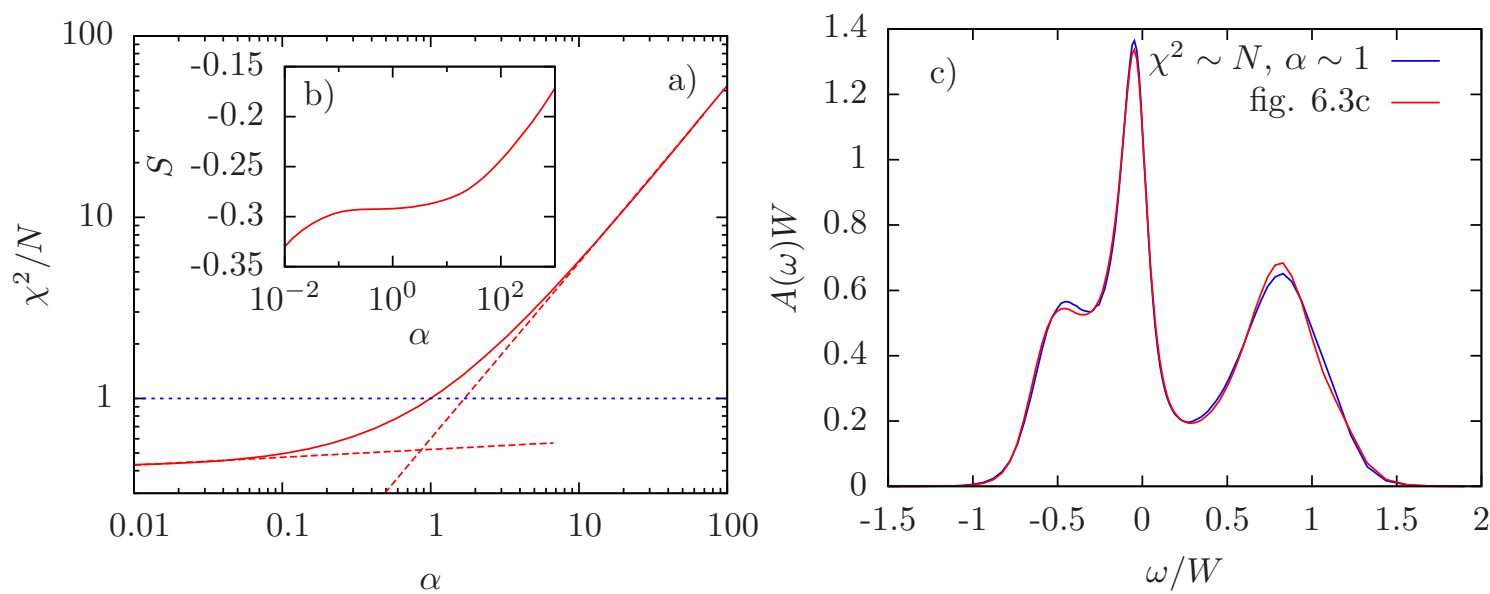

Figure 6.4: The double-logarithmic plot of $\chi^{2}$ (a) shows a kink at $\alpha \approx 0.8$ which is very close to the choice of Syljuåsen $(\alpha=1)$. It is also the region where $\chi^{2} \sim N$. Thus, all three methods give about the same answer and the resulting spectra (c) are identical. A comparison shows that this solution is quite close to the spectrum shown in fig. 6.3c. The entropy (b) exhibits no significant features and gives at least for this data set no indication how to determine $\alpha$.
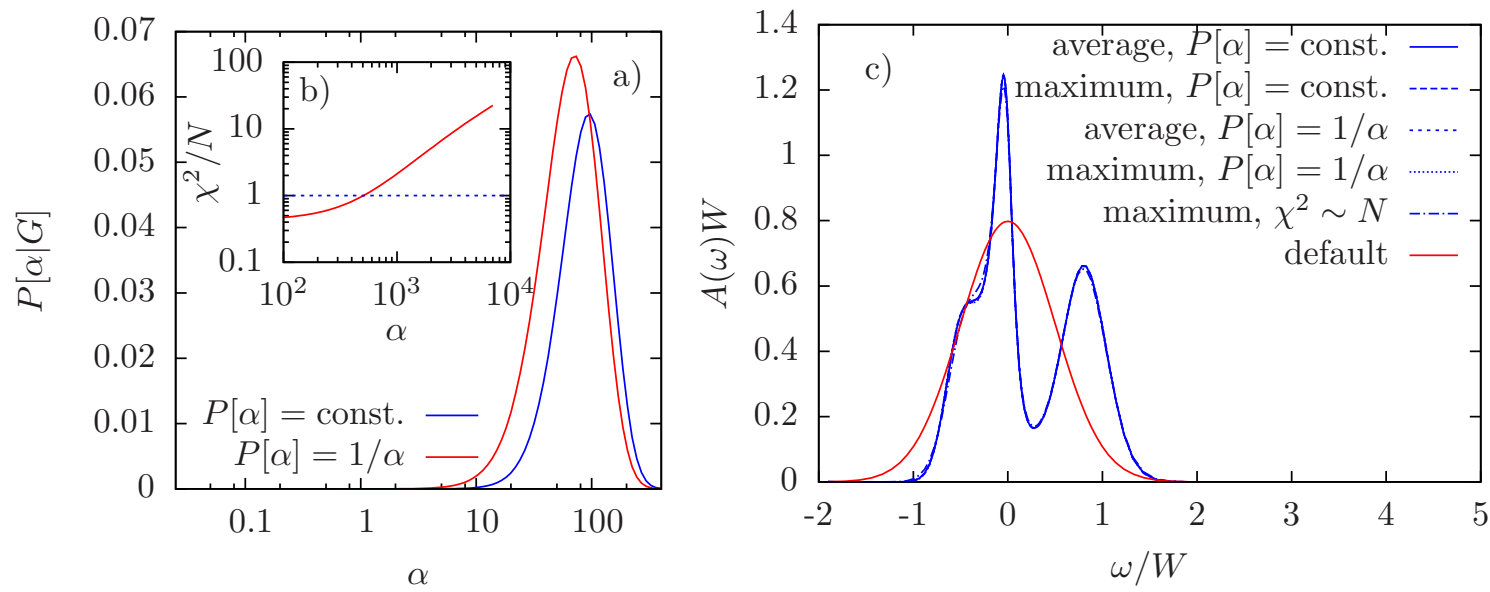

Figure 6.5: Results of a maximum entropy calculation for the same QMC data as in figs. 6.3 and 6.4. The probability distribution $P[\alpha \mid \bar{G}]$ (a) shows a noticeable dependence on $P[\alpha]$, but analogous to SAI, the resulting spectra (c) are identical. The $\alpha$ where $\chi^{2} \sim N($ b) is larger $(\alpha \approx 500)$ than the one for which $P[\alpha \mid \bar{G}]$ is maximal $(\alpha \approx 90)$. Accordingly, the spectrum chosen by the $\chi^{2} \sim N$ rule is more regularized than the one calculated by Bayesian inference. 


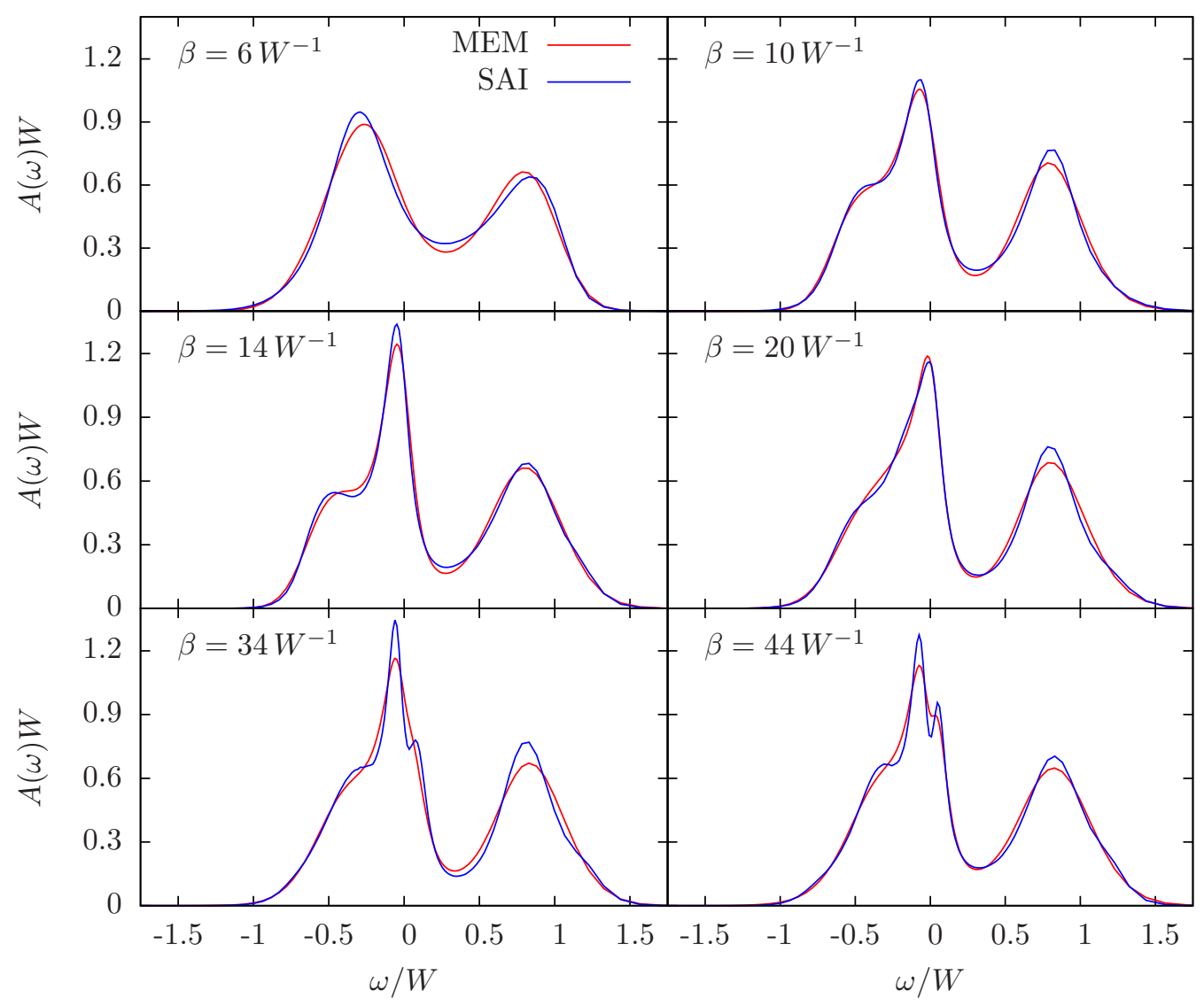

Figure 6.6: Spectra simulated by stochastic analytical inference compared to maximum entropy calculations. All calculations are based on a Gaussian default model.

results of a maximum entropy calculation using Bryan's algorithm [25] for the same QMC data as before. The qualitative behavior is similar to the SAI simulation: the probability distribution $P[\alpha \mid \bar{G}]$ shows a noticeable dependence on the prior probability $P[\alpha]$. However, the resulting spectra depend neither on $P[\alpha]$ nor on whether one averages over $P[\alpha \mid \bar{G}]$ or whether one takes the maximum. The $\chi^{2} \sim N$ rule determines an $\alpha$ which is again larger than the one calculated by Bayesian inference. Accordingly, the spectrum calculated by this criterion is more regularized, although the difference is relatively small. Interestingly, in the MEM the relevant values for $\alpha$ are about one or two orders of magnitude larger compared to those appearing in the SAI simulations. There seems to be no direct correspondence between the $\alpha$-values of the two methods.

An extended comparison of SAI spectra with results of maximum entropy calculations for several temperatures is collected in fig. 6.6. All calculations are based on the Gaussian default model eq. (6.45). As already noted before, MEM tends to 

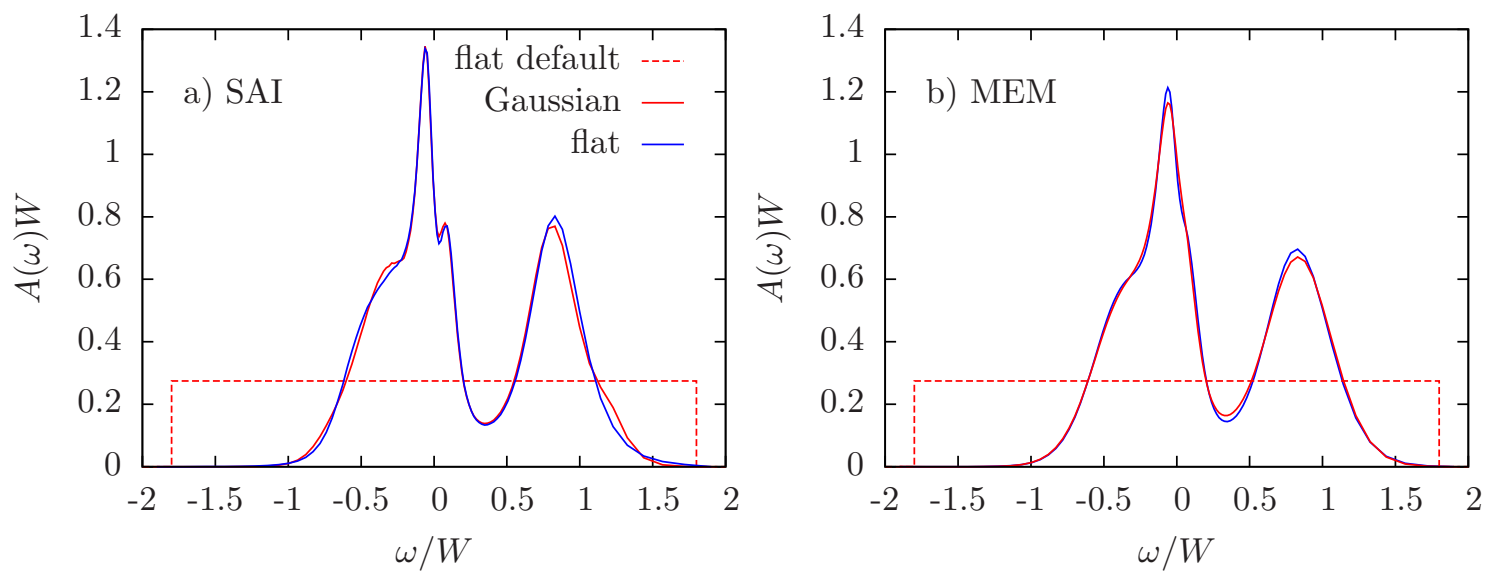

Figure 6.7: Spectra for $\beta=34 W^{-1}$ based on a flat default model calculated by SAI (a) and by the MEM (b). These spectra are only marginally different compared to those calculated using a Gaussian default model. We conclude, that for the QMC data under consideration the calculated spectra are quite independent of the default model.

stronger regularize the spectra and consequently the SAI spectra exhibit noticeably sharper features for all temperatures shown. Especially the pseudo-gap [77], which opens at $\beta=34 W^{-1}$, is captured nicely by SAI while the MEM cannot resolve it yet at that temperature.

An important question concerns the dependence of the spectra on the default model. To this end we show in fig. 6.7 again SAI and the MEM results for the spectrum at $\beta=34 W^{-1}$, this time, however, based on a different default model, namely a rectangular default model of width $1.8 \mathrm{~W}$. The resulting spectra are very similar to the one obtained for the Gaussian default model presented in fig. 6.6. Thus, even at low temperatures the resulting spectra are quite independent of the default model. More precisely, we could not detect a significant default model dependence at any temperature.

Finally, in order to make a definite statement about the accuracy of our method, we test it on a case where the actual spectrum is known. To this end we create artificial input data by constructing a spectrum

$$
A(\omega)=\left\{\begin{array}{ll}
\frac{|\omega|}{\sqrt{\omega^{2}-\frac{1}{4}}}-1 & \text { if }|\omega|>\frac{1}{2} \\
0 & \text { otherwise }
\end{array} .\right.
$$

This function is a particularly difficult example for any analytic-continuation method, since the actually divergent peak is almost impossible to resolve. The spectrum was transformed into fermionic Matsubara frequency space at $\beta=10$ via the integration kernel eq. (6.4). The first 20 frequencies were calculated. Artificial Gaussian distributed random numbers with a standard deviation $\sigma=5 \times 10^{-4}$ where added, 


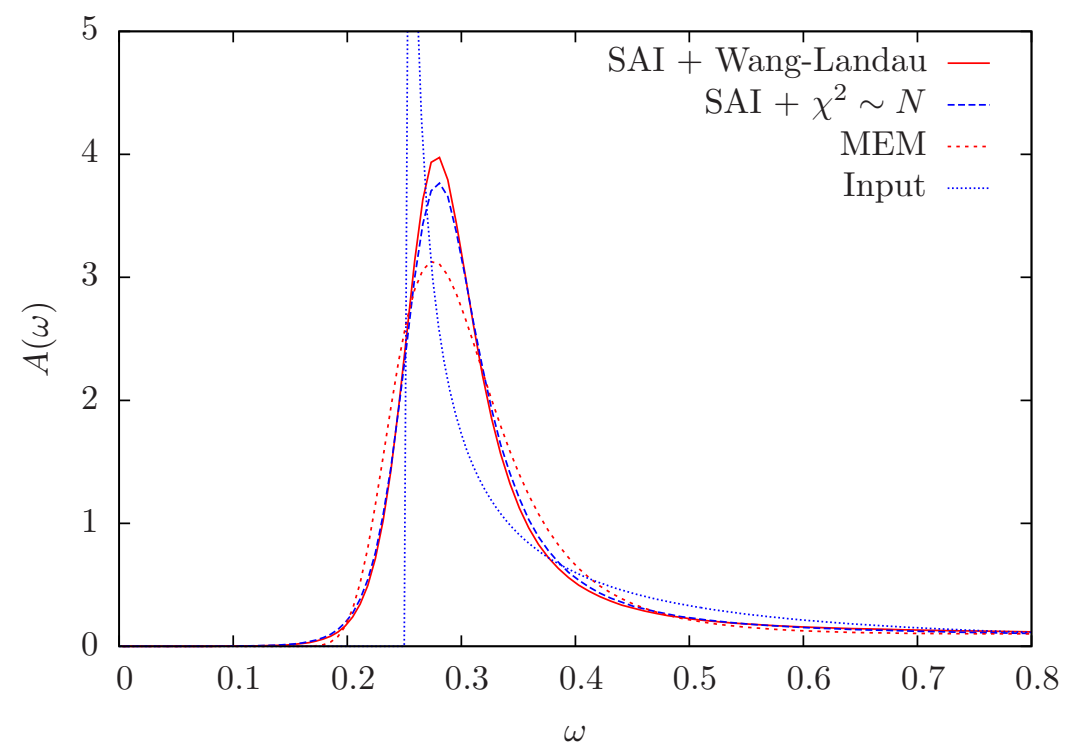

Figure 6.8: Results for a square-root-shaped spectrum. Only positive frequencies are shown since the spectrum is symmetric around zero. This symmetry was enforced for all simulations. The SAI including the Wang-Landau criterion for the determination of $\alpha$ is the most successful in resolving the sharply peaked input spectrum. Note that the choice $\alpha=1$ gives the same result as $\chi^{2}=N$.

simulating a somewhat simplified version of statistical errors of real Monte Carlo data by omitting any correlations between the data points. Figure 6.8 shows that the SAI is more successful in reconstructing the peak than the MEM. It also indicates, that the Wang-Landau criterion leads to an even sharper contour and a better reconstruction of the input spectrum compared to the simple $\chi^{2} \sim N$ rule.

\subsection{Summary}

We have demonstrated that the stochastic analytic-continuation method introduced by SANDVIK and BEACH can be interpreted in terms of Bayesian probability theory. We developed an algorithm that uses Monte Carlo techniques to both calculate the average spectrum and to eliminate the regularization parameter. It treats all probabilities exactly and hereby avoids the approximations made in the maximum entropy method.

Comparisons to the standard MEM show that the SAI results in robust spectral functions which are less regularized and consequently show more pronounced features, in particular with decreasing temperature in the model calculations. As known from standard MEM, no significant dependence on the default model could 
be observed. Comparisons to other approaches to fix the regularization parameter $\alpha$ show that the method identifies a smaller $\alpha$ and thus a typically less regularized spectrum, although for the high-quality Monte Carlo data used here the results differ only slightly. Note that this observation adds additional confidence to the method and this type of analytic continuation in general, because it proves that in the limit of infinitely precise data, all methods give the same result.

A comparison of the Wang-Landau criterion and the $\chi^{2} \sim N$ rule using artificially constructed input data based on a sharply peaked spectrum with a hard gap shows a significant improvement due to the new method; while at the same time the stochastic analytical inference in both cases seems to be more accurate in approximating the singular structure than the classical maximum entropy. As to when simple rules such as $\chi^{2} \sim N$ or the choice $\alpha=1$ may or may not present a good way to fix the regularization parameter depends sensitively on the model, the quality of the data, and structures occurring in the spectral function. However, we can expect the SAI to result in spectra which are in general closer to the exact one, with the Wang-Landau approach typically giving the most accurate image.

One apparent drawback of the method is the necessity to perform simulations for a broad range of values for $\alpha$, independent of whether one chooses the Wang-Landau approach or $\chi^{2} \sim N$, respectively, $\alpha=1$ to fix $\alpha$. Although this can be performed efficiently with parallel-tempering techniques, the required computer resources for one single spectrum can sum up to about 20 processor hours and are hence orders of magnitude larger than the few seconds that are usually needed for standard MEM approaches. Especially for QMC data at higher temperatures, more computer time may even be needed for the analytic continuation than for the simulation of the Monte Carlo data itself. As the resulting spectra tend to be less regularized one has to ponder the gain in details in the structures against the significant increase in computer time. For example, the calculation of momentum-resolved single-particle spectra described in the next chapter required up to 50 analytic-continuation procedures for a single set of parameters. The application of the SAI for this task was therefore not practicable and we reverted to standard MEM techniques. 


\section{Spectral properties of the 3D Hubbard model}

In this chapter, we present momentum-resolved single-particle spectra of the 3D Hubbard model for the paramagnetic and antiferromagnetically ordered phase. The absence of a time discretization error in continuous time QMC and the ability to perform Monte Carlo measurements directly in Matsubara frequencies enable us to analytically continue the self-energies by maximum entropy, which is essential to obtain momentum-resolved spectral functions for the Néel state. We investigate the dependence on temperature and interaction strength and the effect of magnetic frustration introduced by a next-nearest-neighbor hopping. One particular question we address here is the influence of the frustrating interaction on the metal-insulator transition of the 3D Hubbard model.

Major parts of this chapter were published in ref. [44].

\subsection{Method}

Our aim is to study the Hubbard model in three dimensions within the DCA to include both the short to medium-ranged antiferromagnetic fluctuations and the possibility of actual long-range antiferromagnetic order. In the past, the quasistandard for simulations of fermionic many-particle systems was the Hirsch-Fye algorithm [75], which uses a discretization of the imaginary time axis. The absence of a time discretization error in the continuous-time algorithms and the possibility of Monte Carlo measurements directly in Matsubara frequencies enhance the quality of the data significantly [58] and hence enable us to directly analytically continue selfenergies [190]. This avoids the extraction of the self-energies from already continued Green functions by a numerically difficult multi-dimensional root finding algorithm [82].

We obtain from the QMC algorithm the cluster Green function $G_{\sigma K}\left(\mathrm{i} \omega_{n}\right)$. Usually, one then uses the maximum entropy method (MEM) to analytically continue this quantity to the real axis. In order to be able to reverse the coarse-graining, i. e., calculate $G_{\sigma \boldsymbol{k}}\left(\omega+\mathrm{i} 0^{+}\right)$for all $\boldsymbol{k}$ from the first Brillouin zone, one however needs the self-energy $\Sigma_{\sigma \boldsymbol{K}}\left(\omega+\mathrm{i} 0^{+}\right)[109]$. One possible way to extract it is by numerically inverting $G_{\sigma K}\left(\omega+\mathrm{i} 0^{+}\right)$. While this is feasible in the paramagnetic phase, the matrix structure appearing in the antiferromagnetically ordered phase (cf. section 3.5) 
renders this approach impossible.

We therefore follow here an alternative procedure and analytically continue the self-energy directly, which is related to the cluster Green function by

$$
\Sigma_{\sigma \boldsymbol{K}}\left(\mathrm{i} \omega_{n}\right)=\mathcal{G}_{\sigma \boldsymbol{K}}\left(\mathrm{i} \omega_{n}\right)^{-1}-G_{\sigma \boldsymbol{K}}\left(\mathrm{i} \omega_{n}\right)^{-1}
$$

i. e., an inversion of $G_{\sigma \boldsymbol{K}}\left(\mathrm{i} \omega_{n}\right)$. This inverse is calculated directly from the Monte Carlo bins using a jackknife procedure [121], which also incorporates a full error propagation of the covariance matrix. As in all these iterative procedures, the errors of the bare Green function $\mathcal{G}_{\sigma \boldsymbol{K}}\left(\mathrm{i} \omega_{n}\right)$ stemming from previous iterations are neglected. The analytic continuation of the self-energy from imaginary to real frequencies is then performed by the maximum entropy method. Details of the method were already discussed in chapter 6 . Details of the implementation can be found in appendix D.

In order to properly continue self-energies with the MEM, we need to know their high-frequency behavior [190]. To this end, we perform a high-frequency expansion of the self-energy

$$
\Sigma_{\sigma \boldsymbol{K}}\left(\mathrm{i} \omega_{n}\right)=\Sigma_{\sigma}^{0}+\frac{\Sigma_{\sigma}^{1}}{\mathrm{i} \omega_{n}}+\mathcal{O}\left(\left(\mathrm{i} \omega_{n}\right)^{-2}\right)
$$

where the coefficients are given by (see appendix B)

$$
\Sigma_{\sigma}^{0}=U\left(\left\langle n_{\bar{\sigma}}\right\rangle-\frac{1}{2}\right) \quad \text { and } \quad \Sigma_{\sigma}^{1}=U^{2}\left\langle n_{\bar{\sigma}}\right\rangle\left(1-\left\langle n_{\bar{\sigma}}\right\rangle\right)
$$

We now define the quantity

$$
\Sigma_{\sigma K}^{\prime}\left(\mathrm{i} \omega_{n}\right):=\frac{\Sigma_{\sigma K}\left(\mathrm{i} \omega_{n}\right)-\Sigma_{0}}{\Sigma_{1}} .
$$

Since the average number density $\left\langle n_{-\sigma}\right\rangle$ is a Monte Carlo measurement, we estimate $\Sigma_{\sigma \boldsymbol{K}}^{\prime}\left(\mathrm{i} \omega_{n}\right)$ and its covariance matrix by a jackknife procedure as before. The rescaled self-energy $\Sigma_{\sigma K}^{\prime}\left(\mathrm{i} \omega_{n}\right)$ as function of Matsubara frequencies is related to the imaginary part $\operatorname{Im} \Sigma_{\sigma \boldsymbol{K}}^{\prime}(\omega+i \delta)$ on the real frequency axis through the Hilbert transform

$$
\Sigma_{\sigma \boldsymbol{K}}^{\prime}\left(\mathrm{i} \omega_{n}\right)=-\frac{1}{\pi} \int_{-\infty}^{\infty} \mathrm{d} \omega^{\prime} \frac{\operatorname{Im} \Sigma_{\sigma \boldsymbol{K}}^{\prime}\left(\omega^{\prime}\right)}{\mathrm{i} \omega_{n}-\omega^{\prime}}
$$

By virtue of the rescaling eq. (7.4) we furthermore have

$$
-\frac{1}{\pi} \int_{-\infty}^{\infty} \mathrm{d} \omega \operatorname{Im} \Sigma_{\sigma \boldsymbol{K}}^{\prime}(\omega)=1
$$



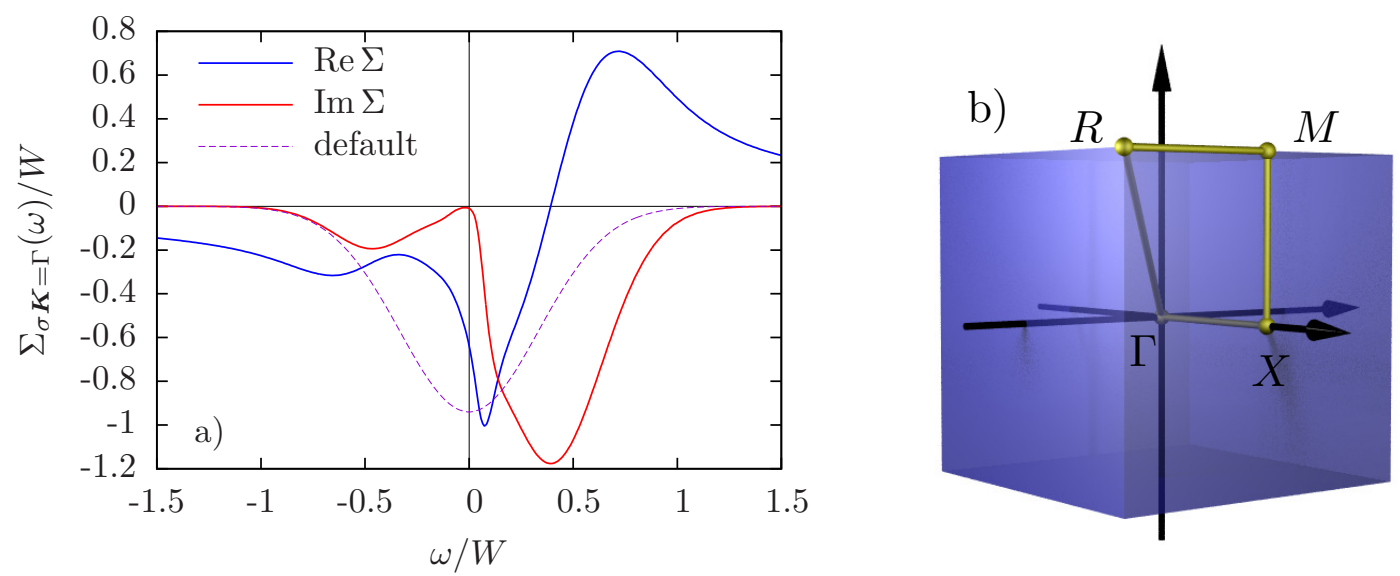

Figure 7.1: The self-energy on the real-frequency axis in the paramagnetic phase for $\boldsymbol{K}=$ $\Gamma, U=W, t^{\prime}=0$ and $T=0.021 \mathrm{~W}$. The default model that entered the MEM calculation of the imaginary part is also shown. The real part is obtained from the imaginary part via eq. (7.7). Panel b: The full first Brillouin zone of the cubic lattice including high-symmetry points and paths taken for plots of $\boldsymbol{k}$ resolved spectra.

i. e., the spectral function $-\frac{1}{\pi} \operatorname{Im} \Sigma_{\sigma \boldsymbol{K}}^{\prime}(\omega)$ is non-negative, normalized to one, and can thus be calculated by the MEM from the data on the imaginary axis. The real part of the self-energy then follows from the Kramers-Kronig relation

$$
\operatorname{Re} \Sigma_{\sigma \boldsymbol{K}}(\omega)=-\frac{1}{\pi} \mathrm{P} \int_{-\infty}^{\infty} \mathrm{d} \omega^{\prime} \frac{\operatorname{Im} \Sigma_{\sigma \boldsymbol{K}}\left(\omega^{\prime}\right)}{\omega-\omega^{\prime}}+\Sigma_{\sigma}^{0}
$$

where P $\int$ denotes a principal value integral. An example for a full self-energy on the real-frequency axis is shown in fig. 7.1. An interpolation of the coarse-grained selfenergies yields the self-energy $\Sigma_{\sigma \boldsymbol{k}}(\omega)$ for all momenta $\boldsymbol{k}$ of the Brillouin zone. We use a 3D interpolation based on Akima splines [2] which provide a smooth interpolation along the momentum points while avoiding unnatural oscillations. Finally, the single particle spectral function $A_{\sigma \boldsymbol{k}}(\omega)$ is calculated using Dyson's equation:

$$
A_{\sigma \boldsymbol{k}}(\omega)=-\frac{1}{\pi} \operatorname{Im} \frac{1}{\omega+\mu-\epsilon_{\boldsymbol{k}}-\Sigma_{\sigma \boldsymbol{k}}(\omega)} .
$$

\subsection{Properties of the paramagnetic phase}

We begin the discussion of our results by presenting spectral functions in the paramagnetic phase of the model, i. e., we will presently deliberately ignore any ordered phase. 


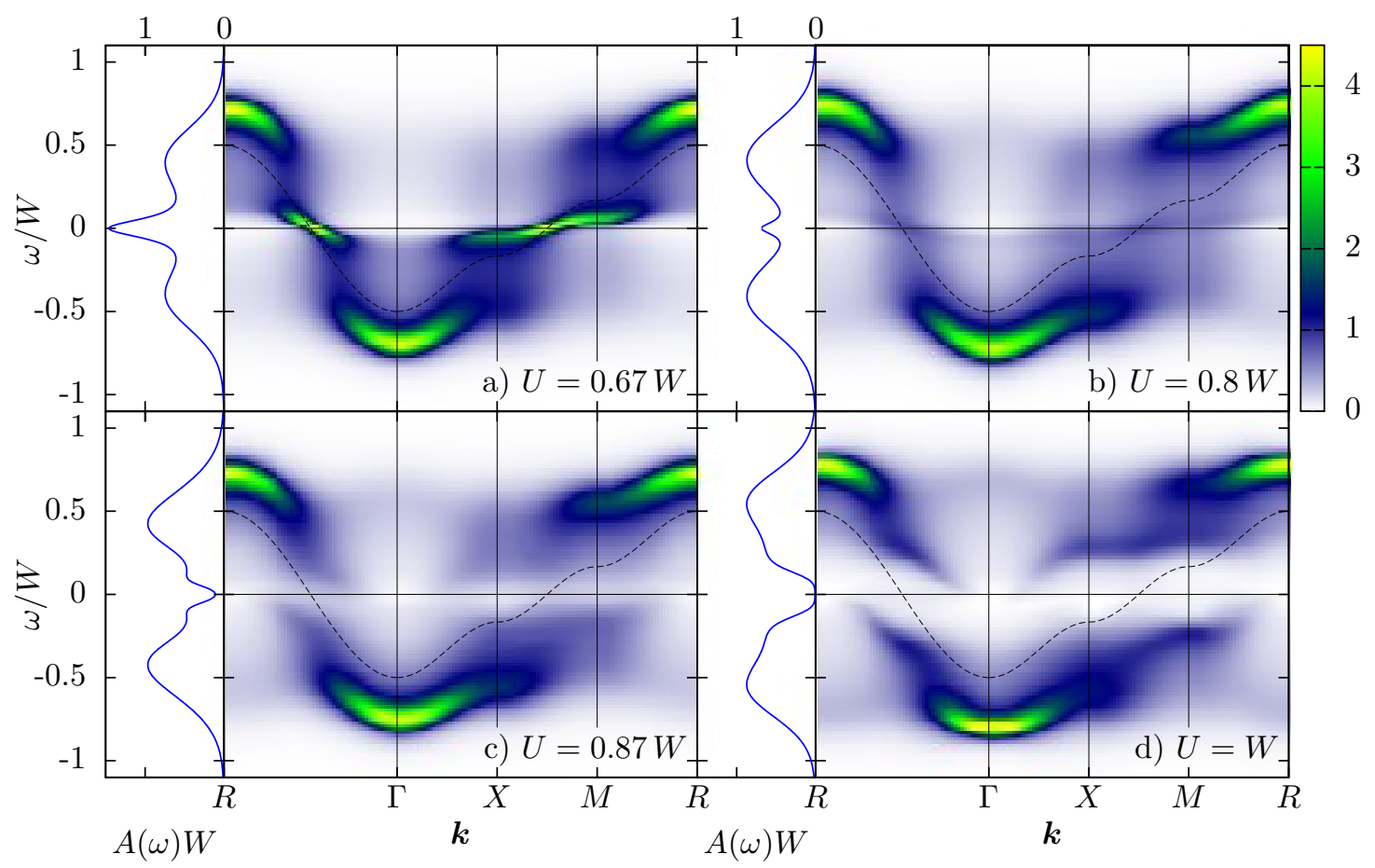

Figure 7.2: Momentum-resolved single-particle spectra $A_{\boldsymbol{k}}(\omega) / \mathrm{W}^{-1}$ for $T=0.021 \mathrm{~W}$ and $t^{\prime}=0$. The momenta $\boldsymbol{k}$ follow a path along high-symmetry directions of the first Brillouin zone as depicted in fig. 7.1b. The left part of each diagram shows the local single-particle spectrum $A(\omega)$ derived from the direct analytic continuation of the Green function. The dashed line denotes the bare dispersion $\epsilon_{\boldsymbol{k}}$.

For this chapter, we choose a fixed cluster of size $N_{\mathrm{c}}=18$, which is described by the vectors $\boldsymbol{a}_{1}=(1,1,2), \boldsymbol{a}_{2}=(2,2,-2)$, and $\boldsymbol{a}_{3}=(2,-1,-1)$. This cluster has been identified as the optimal bipartite cluster of this size [92] following the criteria detailed in section 3.7. Since we are primarily interested in identifying trends and basic physical effects we did not perform calculations for larger clusters to obtain a finite-size scaling as would have been necessary, e.g., for a precise estimation of the Néel temperature in the thermodynamic limit [92]. The high precision necessary for a analytic continuation of the QMC data as well as the relatively low temperatures, especially for simulations around the MH-MIT, rendered a finite-size analysis along the lines of chapter 5 beyond our numerical powers.

\subsubsection{Metallic phase}

Fig. 7.2 presents paramagnetic single particle dispersions for $T=0.021 \mathrm{~W}, t^{\prime}=0$ and different Coulomb repulsions $U$. The selected $\boldsymbol{k}$ points follow a path along 

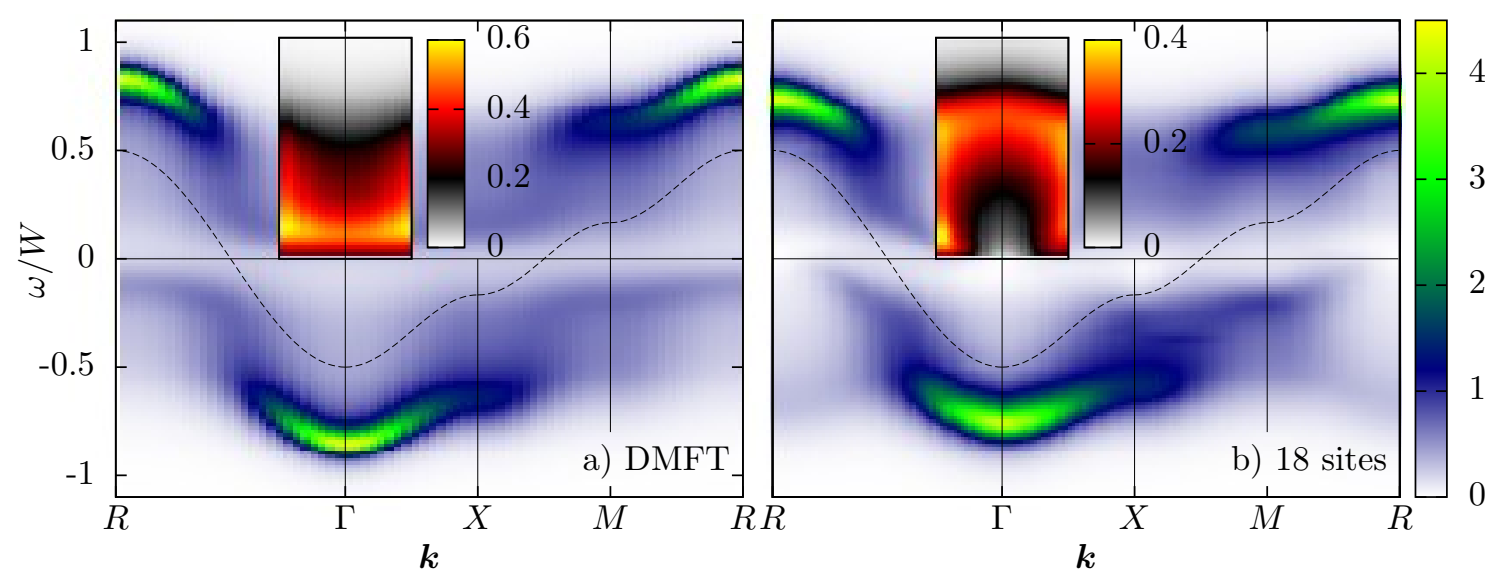

Figure 7.3: Momentum-resolved single-particle spectra $A_{\boldsymbol{k}}(\omega) / W^{-1}$ for $T=0.021 \mathrm{~W}$, $t^{\prime}=0$, and $U=0.93 \mathrm{~W}$ using a non-dispersive DMFT self-energy (a) and a momentum-dependent cluster self-energy (b). The inset highlights a part of the spectrum using an alternative color scheme.

the high-symmetry points of the first Brillouin zone depicted in fig. 7.1b. Local single particle spectra are also shown. For small $U=0.67 \mathrm{~W}$ one observes a quasiparticle peak at the Fermi level, both in the local density of states (DOS) and the momentum-resolved spectral function. The momentum-resolved spectra show, that the main contributions to the quasi-particle peak are situated halfway between the $\Gamma$ and $R$ points and the $X$ and $M$ points, respectively. Comparing its dispersion in these regions to the non-interacting one, one clearly sees a flattening at the Fermi energy, i.e., an increased effective mass of the quasi-particles. At higher energies additional structures - the lower and upper Hubbard bands - are visible, which follow the curvature of the bare dispersion, although shifted to higher energies. The Hubbard bands are connected to the quasi-particle band through broad "waterfall"like features similar to recently observed structures in angel-resolved photoemission spectroscopy of cuprates [50]. For increasing Coulomb repulsion $U$ the quasi-particle band at the Fermi energy vanishes and is replaced by an insulating gap. At the same time the dispersion of the high-energy structures flattens, i. e., the system as a whole becomes more localized in nature. Thus for the temperature under consideration a crossover from a metallic dispersion at $U=0.67 \mathrm{~W}$ to the Mott insulator at $U=W$ is clearly visible. We will return to the details of the Mott-Hubbard metal-insulator transition in section 7.2.2.

In order to make the influence of the momentum dependence of the self-energy in the spectra more transparent, fig. 7.3 compares an insulating spectrum based on self-energies of the 18 site cluster to the corresponding spectrum based on a momentum-independent DMFT self-energy. Although the overall features are similar, there are qualitative differences. For example, if one looks at the details of the 


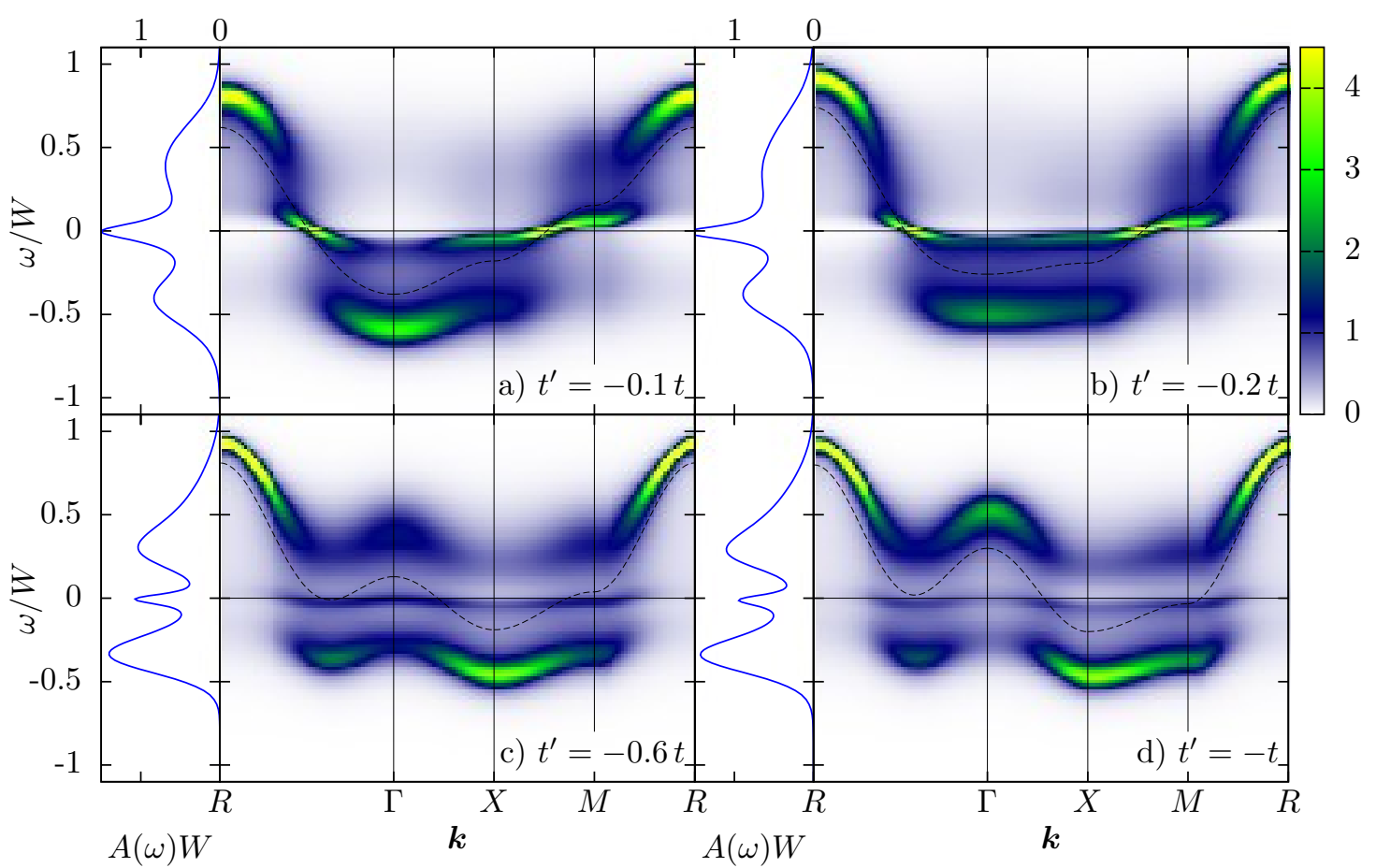

Figure 7.4: Momentum-resolved single-particle spectra $A_{\boldsymbol{k}}(\omega) / W^{-1}$ for $U=0.67 \mathrm{~W}, T=$ $0.021 \mathrm{~W}$ and finite $t^{\prime}$.

spectra close to the $\Gamma$ point (insets to fig. 7.3), one notices that a substantial part of the DMFT spectrum around the $\Gamma$ point is situated just above the Fermi energy. This contribution is shifted to higher frequencies in the cluster calculation, and the curvature is reversed, more resembling the structure of the lower Hubbard band, however with much less spectral weight. This feature can be regarded as a precursor of the complete symmetry with respect to the Fermi energy occurring for spectra in the antiferromagnetically ordered phase (see section 7.3). Thus, we attribute these pale reflections of the lower Hubbard band to the so-called shadow bands [90] arising due to the antiferromagnetic fluctuation neglected by the DMFT simulation.

Next, we want to examine the influence of next-nearest neighbor hopping $t^{\prime}$ on these finding by simulating the system described by the Hamiltonian eq. (2.9). Since we are using QMC to solve the quantum cluster, we have to expect that a finite value of $t^{\prime}$ introduces a fermionic sign problem [184] into the simulations. However, for the temperatures, Coulomb repulsions and cluster sizes studied here the average sign was always greater than 0.94 and thus affects the efficiency of the simulations only weakly.

Fig 7.4 shows results of calculations for $U=0.67 \mathrm{~W}$ and different values for $t^{\prime}$. The particle-hole-symmetric spectrum of fig. 7.2a becomes more and more asymmetric with increasing $t^{\prime}$. These changes can to a large amount be attributed to the changes 


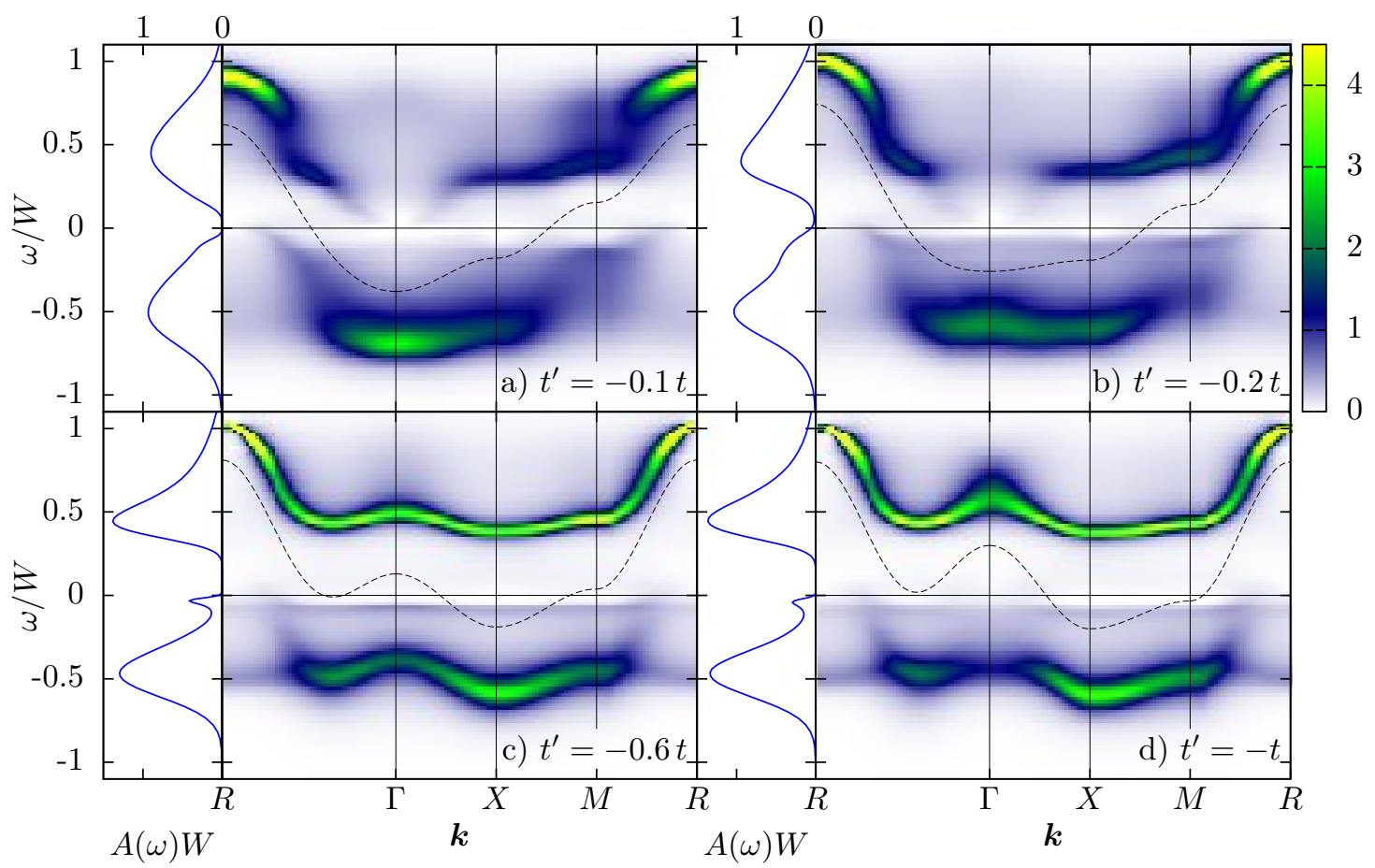

Figure 7.5: Momentum-resolved single-particle spectra $A_{\boldsymbol{k}}(\omega) / W^{-1}$ for $U=W, T=$ $0.021 \mathrm{~W}$ and finite $t^{\prime}$.

in the bare dispersion $\epsilon_{\boldsymbol{k}}$. However, while for small to moderate $t^{\prime}$ the quasi-particle properties do not seem to change dramatically, one observes for larger $t^{\prime}$ a significant reduction in the spectral weight at the Fermi energy, resulting in a reduction of the quasi-particle peak in the DOS. For example, at $t^{\prime}=-t$ the integrated weight of the quasi-particle peak is reduced by $50 \%$ compared to the case with $t^{\prime}=0$ in fig. 7.2a. These observations point towards a reduction of the quasi-particle mass with increasing $t^{\prime}$, in accordance with the findings from DMFT [138].

Frustration effects on the insulating spectrum fig. $7.2 \mathrm{~d}(U=W)$ can be seen in fig. 7.5. As for the metallic spectrum, the features present in fig. $7.2 \mathrm{~d}$ for $t^{\prime}=0$ initially change only weakly, in particular the shadow structures seem to be present, too, albeit reduced in weight. For strong frustration, the Hubbard bands become dominant. Interestingly, these Hubbard bands have a rather well-defined structure and dispersion reminiscent of the bare dispersion. Furthermore, a peak develops just below the Fermi energy, which becomes more pronounced with increasing $t^{\prime}$ and appears to have hardly any momentum dependence. 


\subsubsection{Mott-Hubbard metal-insulator transition}

One of the interesting properties of the Hubbard model is the formation of a correlation driven metal-insulator transition in the paramagnetic phase, the Mott-Hubbard metal-insulator transition (MH-MIT, cf. section 2.1.2). Different from the conventional band insulators for even electron number, where the insulating behavior is due to a completely filled band, the MH-MIT occurs in a partially filled band, which within a simple single-particle picture would thus be conducting. Such a transition is believed to frequently be present in transition metal oxides [78], and has been under debate both from an experimental and theoretical point of view over the past 50 years.

Unfortunately, for a simple cubic lattice with nearest-neighbor hopping only, the MH-MIT of the Hubbard model at half filling is completely covered by the antiferromagnetic phase [144]. Nevertheless, one can study it within a generalized mean-field theory by suppressing any antiferromagnetic order in the system. Under this condition, the MH-MIT can be identified by inspecting the value of the DOS at the Fermi energy: if it is finite at $T=0$, one will have a metal, otherwise an insulator. Identifying it at finite temperature is somewhat more subtle, but again the DOS at the Fermi level can serve as "order parameter". While off the MH-MIT the DOS varies smoothly as function of temperature, it jumps when one approaches the transition. What is even more interesting and has first been shown by the DMFT is that this transition is of first order and quite likely related to an Ising-like transition [48, 123].

An easy way to obtain the trend of the DOS at the Fermi energy without having to perform an analytical continuation is through the low-frequency behavior of $G_{i i}\left(\mathrm{i} \omega_{n}\right)$ : if it decreases towards zero with decreasing $\omega_{n}$, the system is insulating, otherwise metallic. Fig. 7.6a shows the imaginary part of $G_{\text {loc }}\left(i \omega_{n}\right):=G_{i i}\left(i \omega_{n}\right)$ for $t^{\prime}=0$ and $T=0.01 W$ obtained within the DCA for a cluster size of 18 . The jump from an insulating Green function at $U=0.80 \mathrm{~W}$ to a metallic solution at $U=0.766 \mathrm{~W}$ indicates that the MH-MIT is situated in this interval at this temperature. For $T=0.015 \mathrm{~W}$ we could only detect a crossover from insulating to metallic behavior (see fig. 7.6b). This indicates that the critical endpoint of the MH-MIT transition line is located between $T=0.01 W$ and $T=0.015 W$, substantially below the Néel temperature at this interaction strength $\left(T_{\mathrm{N}}=0.030(3) W\right.$ at $\left.U=0.8 W[144,92]\right)$.

A more accurate way to study the MH-MIT is through the effective mass, which is defined as

$$
\frac{m_{\boldsymbol{k}}^{*}}{m}=1-\left.\frac{\partial \operatorname{Re} \Sigma_{\sigma \boldsymbol{k}}(\omega)}{\partial \omega}\right|_{\omega=0},
$$

where $m$ denotes the bare carrier mass. To avoid the process of analytical continuations, the effective mass at finite temperature can be estimated directly from the QMC data as [166]

$$
\frac{m_{\boldsymbol{k}}^{*}}{m} \approx 1-\frac{\operatorname{Im} \Sigma_{\sigma \boldsymbol{k}}\left(\mathrm{i} \omega_{0}\right)}{\omega_{0}}
$$



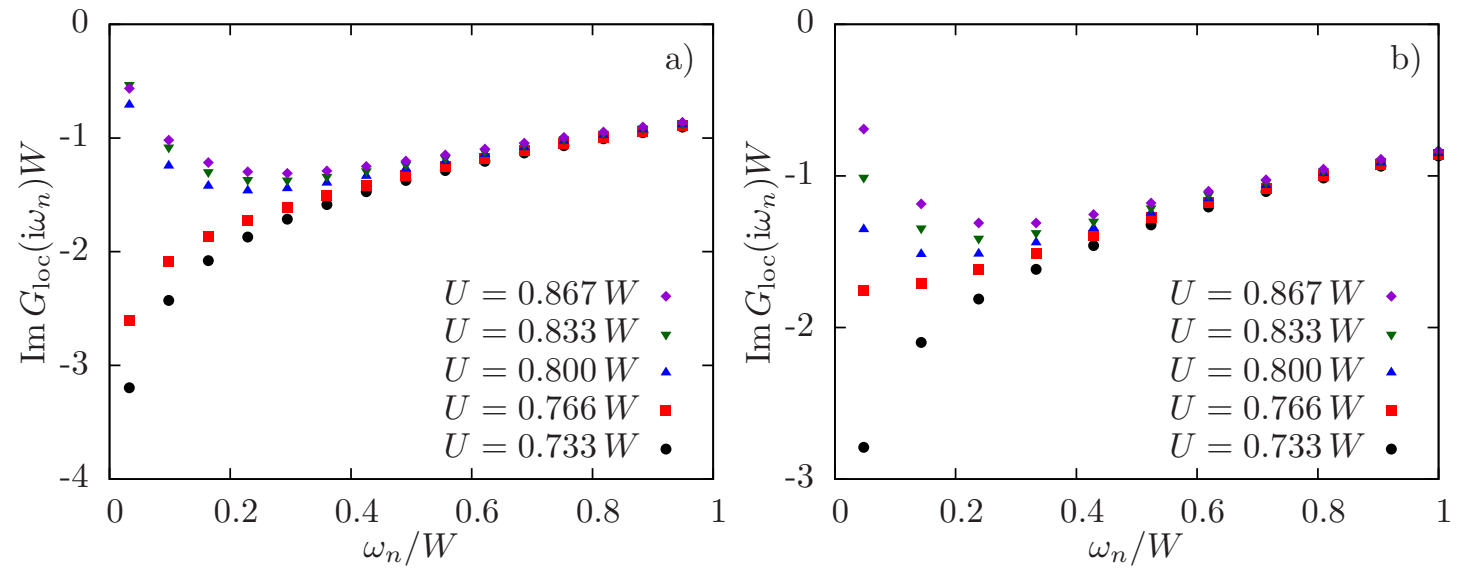

Figure 7.6: The imaginary part of the local Green function in Matsubara frequencies for $t^{\prime}=0, T=0.01 W$ (a) and $T=0.015 W(\mathrm{~b})$. Several values of $U$ around the MH-MIT are shown.

where $\omega_{0}$ is the lowest Matsubara frequency. A sharp increase in $m_{\boldsymbol{k}}^{*}$ across the Fermi surface indicates the metal-insulator transition.

The estimate for the effective mass obtained that way is shown in fig. 7.7a for two different values of $U=0.73 W<U_{c}\left(t^{\prime}=0\right)$ and $U=0.8 W>U_{c}\left(t^{\prime}=0\right)$ respectively $t^{\prime}=0$ and $-0.2 t$ at $T=0.01 \mathrm{~W}$. We do not show the result for $m_{\boldsymbol{k}}^{*}$ from the interpolated data, as the division by $\omega_{0}$ with $\omega_{0} / W \ll 1$ also strongly enhances spurious artificial oscillations due to interpolation, but rather present the masses $m_{\boldsymbol{K}}^{*}$ for each of the 18 cluster momenta as function of their mean distance to the non-interacting Fermi surface for $t^{\prime}=0$. The mean distance is thereby calculated by averaging the distance to the Fermi surface of all points inside the particular cluster cell around $\boldsymbol{K}$. Due to symmetry, some cluster momenta are equivalent and thus we obtain only five different masses. Fig. $7.7 \mathrm{~b}$ depicts one representative cluster momentum for each one of these five equivalence classes.

The first thing one notes is that for both values of $t^{\prime}$ the $\boldsymbol{K}$-dependence of $m_{\boldsymbol{K}}^{*}$ for $U$ well in the metallic phase is rather weak, although nevertheless visible. Going into the insulating phase shows a dramatic increase of the effective mass for the cluster momenta near the Fermi surface. As in particular point 1 is situated on the Fermi surface of the non-interacting system, the natural interpretation is that one preferably obtains strong mass enhancements for $\boldsymbol{k}$-points at or close to the Fermi surface [55]. Points far away from the Fermi surface on the other hand, like points 4 and 5 ( 5 for example corresponding to $\Gamma$ respectively $R$ ), experience only weak renormalizations. This interpretation is further supported by the influence of finite $t^{\prime}$, which moderately reduces the mass.

In the following, we focus on cluster momentum 1, which is situated directly on the Fermi surface, midway between $\Gamma$ and $M$. Since this point exhibits the strongest 

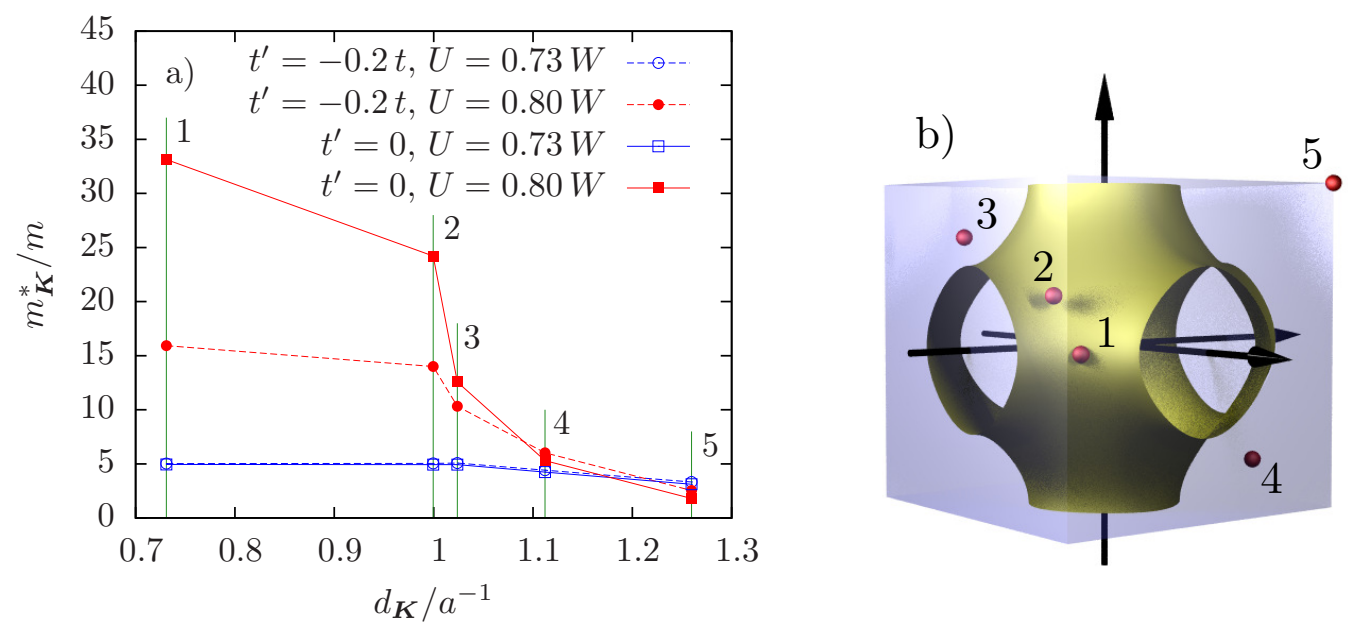

Figure 7.7: The quasi-particle mass $m_{\boldsymbol{K}}^{*}$ as function of mean distance $d_{\boldsymbol{K}}$ from the Fermi surface in units of the lattice spacing $a$ (a). The distance $d_{\boldsymbol{K}}$ is defined as the average of the distances between the Fermi surface and all momenta in the cluster cell described by the cluster momentum $\boldsymbol{K}$. The 18 cluster momenta reduce to 5 different masses due to point symmetries. The labels 1 to 5 refer to the points depicted in panel b, which also shows the non-interacting Fermi surface for $t^{\prime}=0$.

mass enhancement in the insulating phase, it is an ideal candidate to study the MHMIT. The effective mass of this $\boldsymbol{K}$ point is plotted in fig. 7.8 as a function of $U$. At $U_{\mathrm{c}}=0.766 \mathrm{~W}$ both an insulating and a metallic solution can be stabilized, depending on the initial Green function used to start the DCA self-consistency. This behavior indicates a coexistence region in this regime of interaction parameters and tells us that the qualitative physical properties of the paramagnetic MH-MIT do not change at least qualitatively for a true 3D system. The figure also shows the corresponding curves for next-nearest-neighbor hopping parameters $t^{\prime}=-0.1 t$ and $t^{\prime}=-0.2 t$. Here the coexistence region has vanished at the temperature for which the simulations were done, while the relatively smooth shape of the curve indicates that one is still observing a crossover and not yet a sharp phase transition as in the case of $t^{\prime}=0$. This is again in accordance with previous DMFT calculations, where a reduction of the critical temperature and also critical value of $U$ was observed with increasing $t^{\prime}$ [137]. It would be highly desirable to perform simulations at lower temperatures for finite $t^{\prime}$, but as the computational effort necessary increases dramatically with decreasing temperature, we were not yet able to do these simulations for the time being.

Previous studies of the MH-MIT at finite temperatures were typically performed for a Bethe lattice in the limit of infinite dimension within the DMFT approximation $[28,87]$. While the general features of the MH-MIT appear to be rather insensitive to the actual non-interacting band structure, the details like critical values for temper- 


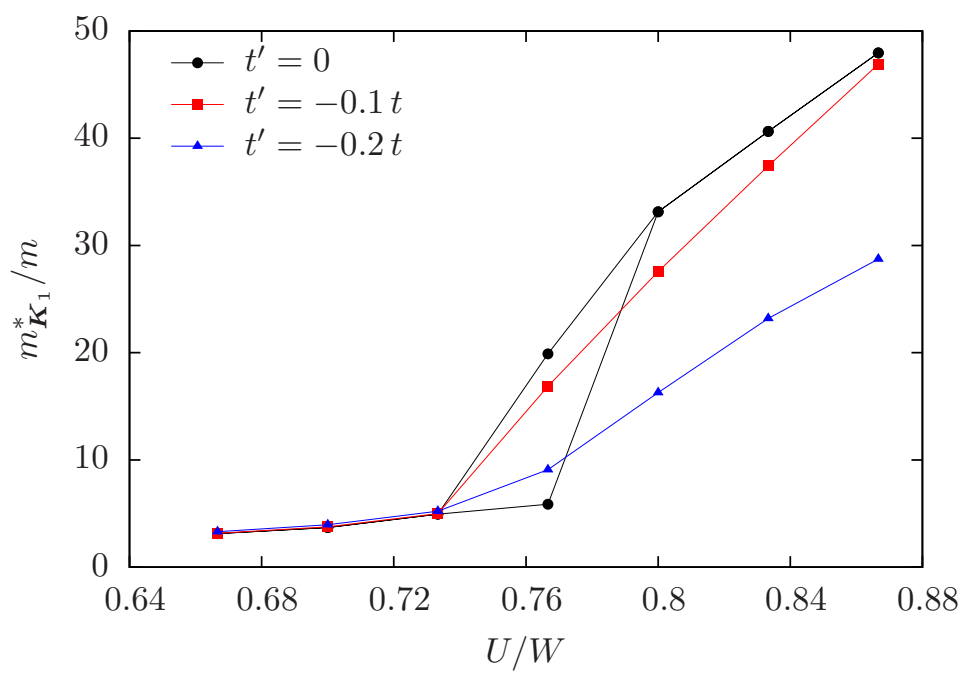

Figure 7.8: The quasi-particle mass estimate $m_{\boldsymbol{K}_{1}}^{*}$ at the midpoint between $\Gamma$ and $M$ (point 1 in fig. 7.7b) for $T=0.01 \mathrm{~W}$ as a function of the interaction $U$.

ature and Coulomb interactions vary strongly with such details. In order to compare these values for lattices with different noninteracting density of states, e. g., the simple cubic lattice, one uses the second moment of the non-interacting density of states $\rho(\omega)[26,48]$,

$$
W_{\mathrm{eff}}=4 \sqrt{\int_{-W / 2}^{W / 2} \mathrm{~d} \omega \omega^{2} \rho(\omega)}
$$

as characteristic energy scale instead of the bandwidth $W$. From eq. (7.11) one obtains $W_{\text {eff }}=W_{\text {Bethe }}$ for the Bethe lattice and $W_{\text {eff }} \approx 0.816 W$ for the simple cubic lattice, and a rather good agreement of critical values when relating them to $W_{\text {eff }}$ $[26,201]$.

Our result $U_{\mathrm{c}}=0.77(3) \mathrm{W}$ for the coexistence region then translates to $U_{\mathrm{c}}=$ $0.94(3) W_{\text {eff }}$ at $T=0.012 W_{\text {eff }}$. For a conventional DMFT calculation, refs. [28] and [87] located the coexistence region for this temperature around $U_{c}=1.18(2) W_{\text {eff }}$ (cf. fig. 2.2). This indicates, that for a true $3 \mathrm{D}$ system the critical values of the MH-MIT will be renormalized, notably the critical $U_{c}$ will be shifted to lower values. Although there is no direct evidence, we think it reasonable to attribute these renormalizations to the short-ranged antiferromagnetic fluctuations present in the DCA. They will have the tendency to suppress the formation of quasi-particles and will thus cause the transition to shift to smaller Coulomb repulsions $U$. 


\subsection{Antiferromagnetic phase}

As already mentioned, the actual thermodynamically stable low-temperature phase of the Hubbard model at half filling and $t^{\prime}=0$ is the antiferromagnet. It also completely covers the MH-MIT [144, 138]. Conventionally, one investigates the onset of the antiferromagnet by looking at the divergence of the staggered susceptibility, coming from the paramagnetic state at high temperature. Another route is to try and simulate the symmetry-broken phase directly. While this scheme surely is not suited to obtain the phase boundaries with high accuracy, it can address the properties within the ordered phase and thus is relevant for comparison to experiment. Furthermore, it is often desirable to investigate the direct change of quantities when one has competing phases or when different ordering phenomena are present simultaneously.

The first thing to note is that the antiferromagnetic order breaks the translational symmetry of the lattice, leading to the well-known doubling of the unit cell. This means, that the first Brillouin zone correspondingly becomes smaller. Evaluating the mapping for the $3 \mathrm{D}$ cubic lattice then results in the reduced zone, also called magnetic Brillouin zone (MBZ) (shown in fig. 2.2a and fig. 7.10b).

As detailed in section 3.5, a small staggered magnetic field is applied to the system during the first iteration of the simulation. The field is switched off for subsequent iterations and the system is allowed to evolve freely. Since the DCA is formulated in momentum space, one has to incorporate the symmetry breaking explicitly by allowing for quantities that are non-diagonal in momentum space. Thus, the antiferromagnetic Green function is represented by the $2 \times 2$ matrix (cf. eq. (3.39))

$$
\begin{aligned}
\mathbf{G}_{\sigma \boldsymbol{K}^{\prime}}\left(\mathrm{i} \omega_{n}\right) & =\left(\begin{array}{ll}
G_{\sigma \boldsymbol{K}^{\prime}}^{00}\left(\mathrm{i} \omega_{n}\right) & G_{\sigma \boldsymbol{K}^{\prime}}^{01}\left(\mathrm{i} \omega_{n}\right) \\
G_{\sigma \boldsymbol{K}^{\prime}}^{10}\left(\mathrm{i} \omega_{n}\right) & G_{\sigma \boldsymbol{K}^{\prime}}^{11}\left(\mathrm{i} \omega_{n}\right)
\end{array}\right) \\
& =\left(\begin{array}{cc}
G_{\sigma \boldsymbol{K}^{\prime}, \boldsymbol{K}^{\prime}}\left(\mathrm{i} \omega_{n}\right) & G_{\sigma \boldsymbol{K}^{\prime}, \boldsymbol{K}^{\prime}+\boldsymbol{Q}}\left(\mathrm{i} \omega_{n}\right) \\
G_{\sigma \boldsymbol{K}^{\prime}+\boldsymbol{Q}, \boldsymbol{K}^{\prime}}\left(\mathrm{i} \omega_{n}\right) & G_{\sigma \boldsymbol{K}^{\prime}+\boldsymbol{Q}, \boldsymbol{K}^{\prime}+\boldsymbol{Q}}\left(\mathrm{i} \omega_{n}\right)
\end{array}\right),
\end{aligned}
$$

where $\boldsymbol{K}^{\prime}$ is an element of the reduced Brillouin zone and $Q=(\pi, \pi, \pi)$. The symmetry relations

$$
G_{\sigma \boldsymbol{K}^{\prime}}^{00}\left(\mathrm{i} \omega_{n}\right)=G_{\bar{\sigma} \boldsymbol{K}^{\prime}}^{11}\left(\mathrm{i} \omega_{n}\right)=-\left(G_{\sigma \boldsymbol{K}^{\prime}}^{11}\left(\mathrm{i} \omega_{n}\right)\right)^{*}=-\left(G_{\bar{\sigma} \boldsymbol{K}^{\prime}}^{00}\left(\mathrm{i} \omega_{n}\right)\right)^{*}
$$

and

$$
G_{\sigma \boldsymbol{K}^{\prime}}^{10}\left(\mathrm{i} \omega_{n}\right)=G_{\sigma \boldsymbol{K}^{\prime}}^{01}\left(\mathrm{i} \omega_{n}\right)=G_{\bar{\sigma} \boldsymbol{K}^{\prime}}^{10}\left(\mathrm{i} \omega_{n}\right)=G_{\bar{\sigma} \boldsymbol{K}^{\prime}}^{01}\left(\mathrm{i} \omega_{n}\right)
$$

hold for the Green function as well as for the self-energy. The latter is still defined via Dyson's equation

$$
\boldsymbol{\Sigma}_{\sigma \boldsymbol{K}^{\prime}}\left(\mathrm{i} \omega_{n}\right)=\mathcal{G}_{\sigma \boldsymbol{K}^{\prime}}\left(\mathrm{i} \omega_{n}\right)^{-1}-\mathbf{G}_{\sigma \boldsymbol{K}^{\prime}}\left(\mathrm{i} \omega_{n}\right)^{-1},
$$

which however now involves quantities which are $2 \times 2$ matrices. 


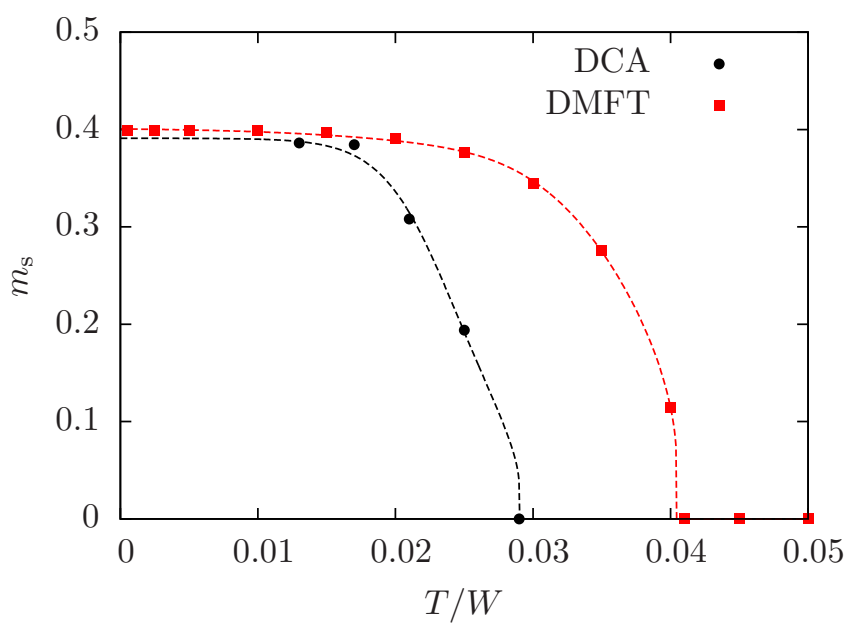

Figure 7.9: Staggered magnetization $m_{s}$ as function of $T$ for $U=0.67 \mathrm{~W}$ as obtained from a DCA calculation with $N_{c}=18$ and the DMFT. The dashed lines are meant as guide to the eye. The DMFT results were obtained by Thomas Pruschke using Wilson's numerical renormalization group algorithm.

A first simple test of the method is to calculate the staggered moment

$$
m_{\mathrm{s}}=\sum_{i} m_{i} \mathrm{e}^{\mathrm{i} \boldsymbol{Q} \cdot \boldsymbol{r}_{i}}
$$

with $m_{i}=n_{i \uparrow}-n_{i \downarrow}$ as a function of temperature and thus locate the antiferromagnetic phase. The results of such a calculation for $U=0.67 \mathrm{~W}$ as function of temperature are shown in fig. 7.9 for DCA simulations and, for comparison, DMFT calculations using Wilson's numerical renormalization group (NRG) algorithm as impurity solver [27]. The first thing to note is that within DCA the critical temperature is reduced by roughly $30 \%$ as compared to the DMFT. The values of $T_{\mathrm{N}} \approx 0.03 W$ for DCA and $T_{\mathrm{N}} \approx 0.042 W$ for DMFT agree with the results obtained by Kent et al. [92]. The saturation value of $m_{\mathrm{s}}(T \rightarrow 0)$, on the other hand, is only weakly affected by the finite cluster. This is in agreement with the expectation, that for a 3D system far enough away from the critical region one should not see dramatic influence by the order parameter fluctuations any more. Finally, while the functional shape of $m_{\mathrm{s}}(T)$ for the DMFT follows the standard mean-field behavior $m_{\mathrm{s}}\left(T \nearrow T_{N}\right) \propto \sqrt{1-T / T_{\mathrm{N}}}$ respectively $m_{\mathrm{s}}(T \rightarrow 0) \propto 1-2 \mathrm{e}^{-2 T_{\mathrm{N}} / T}$, the form obtained from DCA is very different, rather exhibiting a linear behavior just below $T_{\mathrm{N}}$ and a constant value for $T \lesssim 0.02 \mathrm{~W}$.

With the ability to perform reliable calculations in the symmetry-broken phase, one is of course interested in extracting dynamics from the simulations, preferably by analytically continuing the single-particle self-energy. To this end, we again need the high-frequency behavior of the self-energy, which can be obtained from a high- 
frequency expansion (see appendix B) as

$$
\begin{aligned}
\Sigma_{\sigma \boldsymbol{K}^{\prime}}\left(\mathrm{i} \omega_{n}\right)= & \mathcal{O}\left(\left(\mathrm{i} \omega_{n}\right)^{-2}\right)+U\left(\begin{array}{cc}
\left\langle n_{\bar{\sigma}}\right\rangle-\frac{1}{2} & \left\langle m_{\bar{\sigma}}\right\rangle \\
\left\langle m_{\bar{\sigma}}\right\rangle & \left\langle n_{\bar{\sigma}}\right\rangle-\frac{1}{2}
\end{array}\right) \\
& +\frac{U^{2}}{\mathrm{i} \omega_{n}}\left(\begin{array}{cc}
\left\langle n_{\bar{\sigma}}\right\rangle\left(1-\left\langle n_{\bar{\sigma}}\right\rangle\right)+\left\langle m_{\bar{\sigma}}\right\rangle^{2} & \left\langle m_{\bar{\sigma}}\right\rangle\left(1-2\left\langle n_{\bar{\sigma}}\right\rangle\right) \\
\left\langle m_{\bar{\sigma}}\right\rangle\left(1-2\left\langle n_{\bar{\sigma}}\right\rangle\right) & \left\langle n_{\bar{\sigma}}\right\rangle\left(1-\left\langle n_{\bar{\sigma}}\right\rangle\right)+\left\langle m_{\bar{\sigma}}\right\rangle^{2}
\end{array}\right)
\end{aligned}
$$

using the staggered spin polarization

$$
\left\langle m_{\sigma}\right\rangle=\sum_{i} \mathrm{e}^{\mathrm{i} \boldsymbol{Q} \cdot \boldsymbol{r}_{i}}\left\langle n_{\sigma i}-n_{\bar{\sigma} i}\right\rangle
$$

The direct analytic continuation of non-diagonal self-energies - or Green functions - is not possible, since the non-diagonal spectral function $-\frac{1}{\pi} \operatorname{Im} \Sigma_{\sigma \boldsymbol{K}^{\prime}}^{10}(\omega)$ has both negative and positive values while the standard MEM algorithm can only deal with non-negative spectral functions. In order to solve this problem, we employ the linear transformation [180] (omitting spin and frequency dependencies)

$$
\Sigma_{\boldsymbol{K}^{\prime}}^{ \pm}=\frac{\Sigma_{\boldsymbol{K}^{\prime}}^{00}+\Sigma_{\boldsymbol{K}^{\prime}}^{11}}{2} \pm \Sigma_{\boldsymbol{K}^{\prime}}^{10}
$$

and determine $\operatorname{Im} \Sigma_{\sigma \boldsymbol{K}^{\prime}}^{ \pm}(\omega)$ along the diagonal elements $\operatorname{Im} \Sigma_{\sigma \boldsymbol{K}^{\prime}}^{00}(\omega)$ and $\operatorname{Im} \Sigma_{\sigma \boldsymbol{K}^{\prime}}^{11}(\omega)$ using the MEM. Analogous to the paramagnetic case, the high-frequency coefficients eq. (7.17) are used to normalize the self-energies prior to the analytic continuation. Finally, the real parts are calculated by a Kramers-Kronig relation analogous to eq. (7.7). Since the transformation eq. (7.19) is linear, it holds for the analytically continued functions as well and can thus be solved for the non-diagonal element $\Sigma_{\sigma K^{\prime}}^{10}(\omega)$. An example for a complete self-energy matrix on the real frequency axis obtained by this procedure is shown in fig. 7.10. Note that the diagonal and offdiagonal elements have different symmetry properties. For the particle-hole symmetric situation presented here, the former obey the relation $\Sigma_{\sigma \boldsymbol{K}^{\prime}}^{\alpha \alpha}(\omega)=\sum_{\bar{\sigma} \boldsymbol{K}^{\prime}}^{\alpha \alpha}(-\omega)$ respectively $\sum_{\sigma \boldsymbol{K}^{\prime}}^{\bar{\alpha} \bar{\alpha}}(\omega)=\sum_{\sigma \boldsymbol{K}^{\prime}}^{\alpha \alpha}(-\omega)$ following from the structure of the Néel state, while the latter are all identical but obey $\Sigma_{\sigma \boldsymbol{K}^{\prime}}^{\alpha \bar{\alpha}}(\omega)=-\Sigma_{\bar{\sigma} \boldsymbol{K}^{\prime}}^{\alpha \bar{\alpha}}(-\omega)$. This last relation in particular implies that the real part is an even function of $\omega$, and the imaginary part is odd.

The resulting self-energies for the cluster $\boldsymbol{K}^{\prime}$ points are then interpolated as in the paramagnetic case, and finally the spin-averaged spectral function for all momenta $\boldsymbol{k}^{\prime}$ of the magnetic Brillouin zone follows from

$$
A_{\boldsymbol{k}^{\prime}}(\omega)=-\frac{1}{\pi} \operatorname{Im} \operatorname{Tr}\left[\left(\begin{array}{lr}
\omega+\mu-\epsilon_{\boldsymbol{k}^{\prime}} & 0 \\
0 & \omega+\mu-\epsilon_{\boldsymbol{k}^{\prime}+\boldsymbol{Q}}
\end{array}\right)-\boldsymbol{\Sigma}_{\boldsymbol{k}^{\prime}}(\omega)\right]^{-1},
$$

where $\operatorname{Tr}$ denotes the trace over the $2 \times 2$-matrix. 

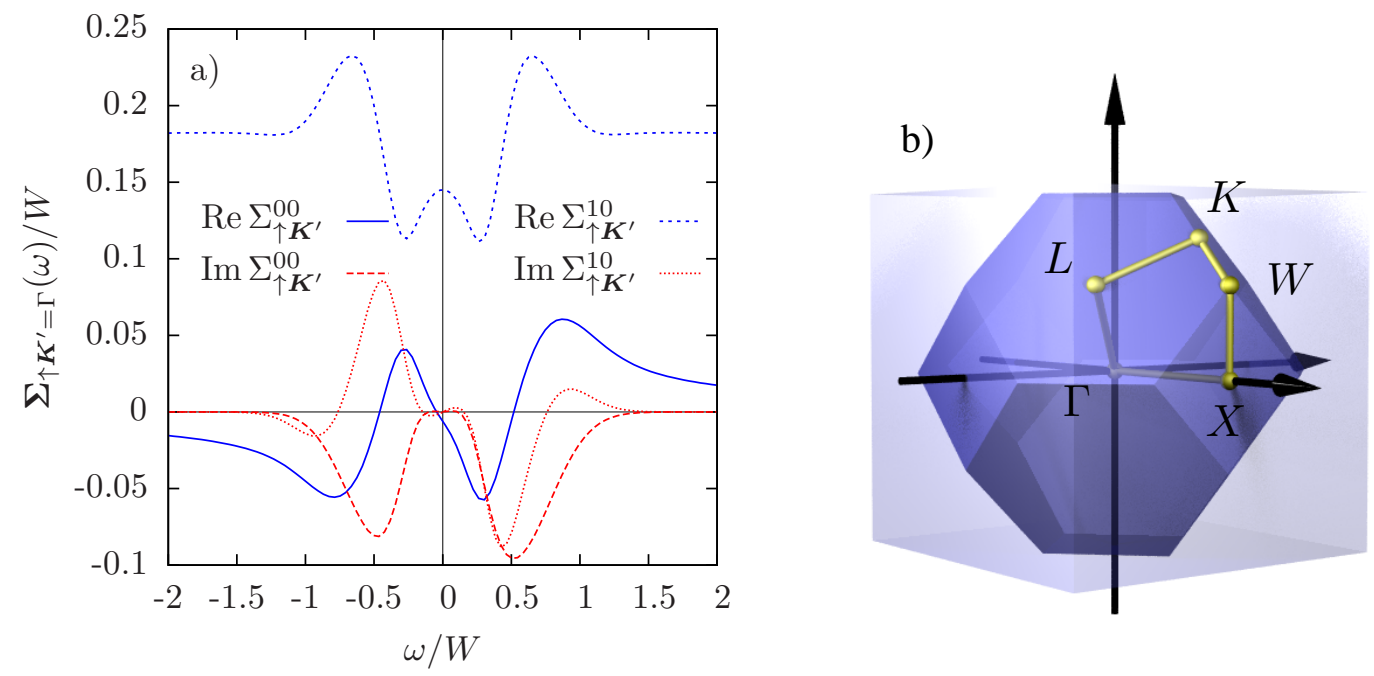

Figure 7.10: The self-energy for $\boldsymbol{K}^{\prime}=\Gamma, U=0.5 \mathrm{~W}, t^{\prime}=0$ and $T=0.021 \mathrm{~W}$ in the antiferromagnetic phase (a). The real and imaginary parts of the elements $\Sigma_{\uparrow \boldsymbol{K}^{\prime}}^{00}(\omega)=\Sigma_{\downarrow \boldsymbol{K}^{\prime}}^{11}(\omega)=\Sigma_{\uparrow \boldsymbol{K}^{\prime}}^{11}(-\omega)=\Sigma_{\downarrow \boldsymbol{K}^{\prime}}^{00}(-\omega)$ and $\Sigma_{\uparrow \boldsymbol{K}^{\prime}}^{10}(\omega)=\Sigma_{\uparrow \boldsymbol{K}^{\prime}}^{01}(\omega)=$ $\Sigma_{\downarrow \boldsymbol{K}^{\prime}}^{10}(\omega)=\Sigma_{\downarrow \boldsymbol{K}^{\prime}}^{01}(\omega)$ are shown. The right panel (b) depicts the magnetic Brillouin zone of the simple cubic lattice and the path along high-symmetry points used for the presentation of spectra in the antiferromagnetically ordered state in fig. 7.11 .

Results for single-particle spectra in the antiferromagnetically ordered phase and different values of $U$ for $T=0.021 \mathrm{~W}$ are shown in fig. 7.11a-c for paths connecting high-symmetry points in the magnetic Brillouin zone in fig. 7.10b. As expected, the spectral function and DOS have a gap around the Fermi energy, i. e., we always have an insulating state. Furthermore, it is symmetric with respect to the Fermi energy, reflecting the back-folding of the spectrum due to the broken translational symmetry. Along a large part of the Brillouin zone one has rather flat bands. For weak and moderate coupling these structures have a rather high spectral weight, which results in the formation of characteristic van Hove singularities at the gap edges. This is a typical weak-coupling result consistent with a conventional Hartree approximation. The gap increases for increasing $U$, while at the same time the weight in the structures at the gap edges is redistributed to larger energies, leading to a softening of the structures in the DOS.

Fig. 7.11d shows an antiferromagnetic spectrum for finite $t^{\prime}=-0.2 t$. The nextnearest-neighbor hopping breaks the symmetry with respect to the Fermi energy analogous to the paramagnetic case. Since magnetically frustrating interactions cause the antiferromagnetic phase to vanish quickly at the present temperature, no larger value of $t^{\prime}$ was simulated. 


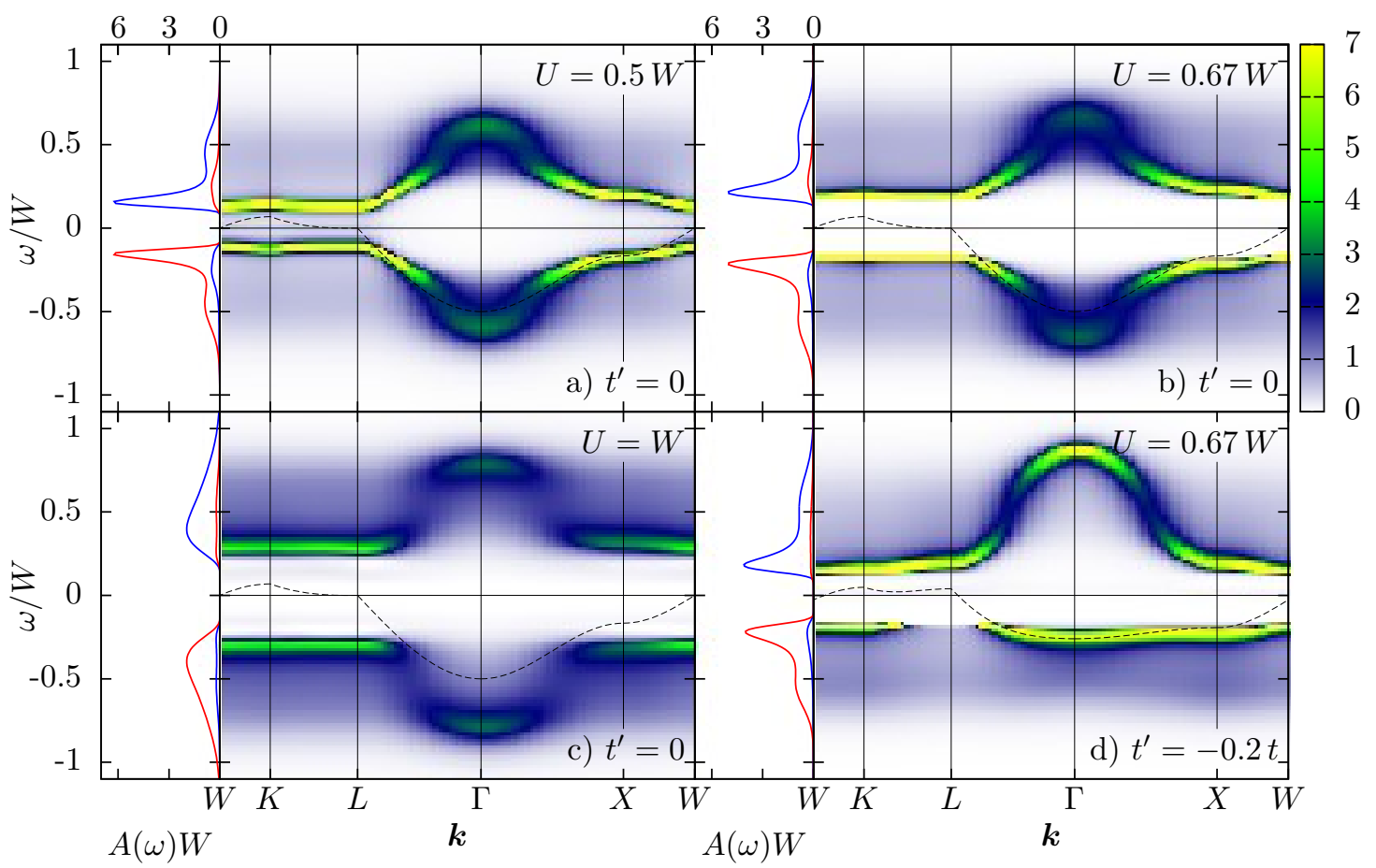

Figure 7.11: Spin-averaged single-particle spectra $A_{\boldsymbol{k}}(\omega) / W^{-1}$ for $T=0.021 \mathrm{~W}$ in the antiferromagnetic phase for different interaction strengths. The lower right panel (d) shows a spectrum for finite next-nearest-neighbor hopping $t^{\prime}=$ $-0.2 t$. The left part of each figure depicts the local single-particle spectrum for both the majority spins (red) and the minority spins (blue). In figure (a) the edges of the gap are too sharp to resolve properly. In order to avoid a numerical division by zero, an artificial imaginary shift $-\mathrm{i} \delta$ with $\delta=0.03 \mathrm{~W}$ was added to the self-energy. The result is a slight broadening of the gap edges. The interpolation follows the path along the high-symmetry points of the reduced Brillouin zone depicted in fig. 7.10b.

\subsection{Summary}

One particular drawback of QMC algorithms is the problem to extract spectral functions from the QMC data. As this requires an analytic continuation based on maximum entropy, a thorough error analysis for the quantity to be continued including proper high-frequency information is necessary. With the standard HirschFye QMC this prevented the direct continuation of the irreducible self-energy and made rather unreliable root-searching techniques necessary [82].

Modern continuous-time QMC algorithms allow for a direct simulation of data in frequency space and moreover yield high-quality data for the self-energy with reliable error estimates, thus allowing for a direct analytic continuation of the self-energy. 
Based on this new route we presented a method to extract momentum-resolved dynamical correlation functions from QMC simulations of strongly correlated electron systems. We showed that QMC simulation of clusters within the DCA can provide data which enable the calculation of both momentum and frequency-resolved singleparticle spectra. The method can resolve very detailed structures in the spectral functions, including waterfall-like features. Thus, spectra based on momentumresolved self-energies can lead to qualitatively different features as compared to DMFT spectra, and will give a more reliable way to extrapolate cluster results.

In addition, we showed that one now can access not only spectra in the paramagnetic state but is also able to calculate frequency and momentum-resolved spectra in more complex ordered phases. As an example, we discussed spectral properties of the Hubbard model inside the antiferromagnetic phase, also including an additional magnetic frustration through next-nearest-neighbor hopping.

We detected the MH-MIT at $T=0.012 W_{\text {eff }}$ and $U_{c}=0.94(3) W_{\text {eff }}$, a value, which is substantially smaller than the DMFT result $U_{\mathrm{c}}=1.18(2) W_{\text {eff }}$. For finite values of $t^{\prime}$ no MH-MIT could be detected for temperatures $T \geq 0.01 \mathrm{~W}$. The presence of a smooth cross-over indicates that the transition has presumably moved to lower temperatures - an effect that has been previously studied in a DMFT context [137]. To clarify this point as well as the $t^{\prime}$ dependence of $U_{\mathrm{c}}$, further studies at lower temperatures are necessary. 


\section{Summary}

We have studied the Hubbard model in three dimensions within the dynamical cluster approximation using quantum Monte Carlo simulations and analytic-continuation techniques.

We have provided the full thermodynamics of the 3D Hubbard model in the thermodynamic limit for $U \leq W$ and temperatures above the Néel temperature in chapter 5 . The necessary finite-size scaling analysis relied on large-scale cluster calculations up to $N_{\mathrm{c}}=100$ cluster sites which would not have been numerically feasible without the recently developed highly-optimized continuous-time QMC algorithms. Comparing the DCA results to single site DMFT, we found that the latter already fails at remarkably high temperatures near half filling. This indicates that DMFT extensions such as the DCA - although numerically much more expensive are mandatory for a precise quantitative study of the Hubbard model.

We have made contact to optical lattice experiments by calculating entropy averages over realistic trap geometries thereby providing an exact estimate of the entropy which would be sufficient to reach an antiferromagnetically ordered state in the center of the optical trap. The calculated value is $\sim 40 \%$ larger than the entropy at Néel temperature in a homogeneous system showing the trap geometry to be an important influence on the system. We have also investigated the double occupancy and the nearest-neighbor spin-spin correlation function. The double occupancy was shown to be almost flat as a function of temperature, while the spin correlations have shown a strong temperature dependence around the Néel temperature. This suggests that the spin correlations, not the double occupancy, are best suited to observe precursors of antiferromagnetism and measure the temperature. Our data can be used to calibrate such a spin-correlation thermometer and could be experimentally valuable for the detection of the antiferromagnetic phase.

We have addressed the difficult and even ill-posed problem of extracting energy spectra from QMC simulations (chapter 6). After giving an overview of the maximum entropy method (MEM) that is usually applied to this problem, we have introduced stochastic analytical inference as an extension of the stochastic analyticcontinuation method introduced by SANDVIK and BEACH which is fully based on Bayesian probability theory. We developed an algorithm that uses Monte Carlo techniques to both calculate an average over all possible spectra and to eliminate the characteristic regularization parameter of analytic-continuation algorithms. It treats all probabilities exactly and hereby avoids the approximations made in the maximum entropy method. Comparisons to the MEM have shown that the SAI produces 
robust spectral functions which are less regularized and display more pronounced features compared to MEM spectra. Since maximum entropy methods provide general data analysis tools whose application is not limited to analytic-continuation techniques [94], our research in this field could also be relevant in various other fields of science and engineering.

We have demonstrated the possibility to extract both momentum and frequencyresolved single-particle spectra from simulation of clusters within the DCA in chapter 7. The recently developed continuous-time QMC algorithms have provided highquality data that allowed for a direct analytic continuation of self-energies. The method was able to resolve detailed structures in the spectral functions which lead to qualitatively different features as compared to DMFT spectra. We have shown that this method can be extended to symmetry-broken phases and discussed energy spectra inside the antiferromagnetic phase, also including magnetic frustration. Additional simulations detected the Mott-Hubbard metal-insulator transition at substantially smaller value for the Coulomb interaction than was previously calculated by DMFT.

We have studied the Hubbard model focussing on two aspects, thermodynamics and spectral functions. On the one hand, the DCA in combination with highperformance QMC techniques offered the possibility of a systematic finite-size scaling analysis and was able to produce precise results for the thermodynamic limit of the system. On the other hand, the calculation of energy spectra - although inherently difficult - benefited from recent development in both QMC and analytic-continuation methods producing additional insight into the physical properties of the model. Thus, we have shown that the DCA in combination with modern QMC and analyticcontinuation methods constitute valuable numerical tools to study thermodynamic and spectral properties of quantum many-particle systems. 


\section{Appendix A}

\section{CT-INT: Inversion by partitioning}

The CT-INT algorithm introduced in chapter 4 requires the efficient calculation of ratios of determinants (cf. eqs. (4.32), (4.33), and (4.37))

$$
r=\frac{\operatorname{det} M_{k+1}^{-1}}{\operatorname{det} M_{k}^{-1}},
$$

where $M_{k}^{-1}$ denotes a $k \times k$ matrix. The matrix $M_{k+1}^{-1}$ is the matrix $M_{k}^{-1}$ with one additional row and column containing either - in an insertion update - the newly added vertex, or the vertex to be deleted in an removal update. Since the determinant is invariant under the exchange of rows and columns, we assume these additional row and column to be the last ones, writing

$$
M_{k+1}^{-1}=\left(\begin{array}{cc}
M_{k}^{-1} & Q \\
R & S
\end{array}\right)
$$

where $Q, R, S$ are $k \times 1,1 \times k$, and $1 \times 1$ matrices, respectively, which contain the additional row and column. It has been proven to be most efficient to store the inverse matrix $M_{k}$, mostly because all measurements depend on $M_{k}$ instead of $M_{k}^{-1}$. This causes also the question how to update the matrix during an accepted Monte Carlo update while avoiding a numerically expensive inversion of the whole matrix. In other words, knowing $M_{k}, Q, R$, and $S$, how do we compute

$$
M_{k+1}=\left(\begin{array}{cc}
\tilde{P} & \tilde{Q} \\
\tilde{R} & \tilde{S}
\end{array}\right) ?
$$

Following ref. [186], a straightforward calculation shows that the solution to this problem is

$$
\begin{aligned}
\tilde{S} & =\left(S-R M_{k} Q\right)^{-1} \\
\tilde{Q} & =-M_{k} Q \tilde{S} \\
\tilde{R} & =-\tilde{S} R M_{k} \\
\tilde{P} & =M_{k}+M_{k} Q \tilde{S} R M_{k} .
\end{aligned}
$$


Only one inversion in the calculation of $\tilde{S}$ is needed, which is trivial because $\tilde{S}$ is only a $1 \times 1$ matrix. For the rest of the update $\mathcal{O}\left(k^{2}\right)$ steps are necessary for the matrix multiplications. Using $\operatorname{det} M=1 / \operatorname{det} M^{-1}$ and the Laplace expansion for determinants, the ratio eq. (A.1) can also be determined yielding

$$
r=\frac{\operatorname{det} M_{k}}{\operatorname{det} M_{k+1}}=\frac{1}{\operatorname{det} \tilde{S}}=\operatorname{det}\left[S-R M_{k} Q\right]
$$

This expression is the basis of the measurement formula eq. (4.39). The presented formalism does not only hold for one additional row and column in the determinant ratio eq. (A.1). It is also valid for the case where $S$ is a general $n \times n$ matrix, $Q$ a $k \times n$ matrix, and $R$ an $n \times k$ matrix, respectively [186]. Thus, the relation

$$
\frac{\operatorname{det} M_{k}}{\operatorname{det} M_{k+2}}=\operatorname{det}\left[S-R M_{k} Q\right]
$$

can be applied to the measurement of two-particle correlations as in eq. (4.42). In contrast to eq. (A.8), however, the result is the determinant of a $2 \times 2$ matrix. 


\section{Appendix B}

\section{High-frequency expansion}

In this appendix chapter, the high-frequency tails of Green functions and selfenergies obtained for the dynamical mean-field theory [141, 35] are generalized to the momentum-dependent case suitable for the DCA in both antiferromagnetic and paramagnetic phases.

The antiferromagnetic coarse-grained Green function $\overline{\mathbf{G}}_{\sigma \boldsymbol{K}^{\prime}}\left(\mathrm{i} \omega_{n}\right)$ in the presence of a staggered magnetic field can be described by (see eq. (3.41))

$$
\overline{\mathbf{G}}_{\sigma \boldsymbol{K}^{\prime}}\left(\mathrm{i} \omega_{n}\right)=\frac{1}{V} \int \mathrm{d} \tilde{\boldsymbol{k}}\left[\left(\begin{array}{lrr}
\mathrm{i} \omega_{n}-\xi_{\boldsymbol{K}^{\prime}+\tilde{\boldsymbol{k}}} & h_{\sigma} / 2 \\
h_{\sigma} / 2 & \mathrm{i} \omega_{n}-\xi_{\boldsymbol{K}^{\prime}+\boldsymbol{Q}+\tilde{\boldsymbol{k}}}
\end{array}\right)-\boldsymbol{\Sigma}_{\boldsymbol{K}^{\prime}}\left(\mathrm{i} \omega_{n}\right)\right]^{-1}
$$

using the matrix notation eq. (7.12) and $\xi_{\boldsymbol{K}^{\prime}}=\epsilon_{\boldsymbol{K}^{\prime}}-\mu$. In order to gain an expression for the high-frequency coefficients of the self-energy, one uses the ansatz

$$
\Sigma_{\sigma \mathbf{K}^{\prime}}\left(\mathrm{i} \omega_{n}\right)=\Sigma_{\sigma \boldsymbol{K}^{\prime}}^{0}+\frac{\boldsymbol{\Sigma}_{\sigma \boldsymbol{K}^{\prime}}^{1}}{\mathrm{i} \omega_{n}}+\mathcal{O}\left(\left(\mathrm{i} \omega_{n}\right)^{-2}\right)
$$

and expands the coarse-grained Green function up to fourth order:

$$
\overline{\mathbf{G}}_{\sigma \boldsymbol{K}^{\prime}}\left(\mathrm{i} \omega_{n}\right)=\frac{\mathbf{C}_{\sigma \boldsymbol{K}^{\prime}}^{1}}{\mathrm{i} \omega_{n}}+\frac{\mathbf{C}_{\sigma \boldsymbol{K}^{\prime}}^{2}}{\left(\mathrm{i} \omega_{n}\right)^{2}}+\frac{\mathbf{C}_{\sigma \boldsymbol{K}^{\prime}}^{3}}{\left(\mathrm{i} \omega_{n}\right)^{3}}+\mathcal{O}\left(\left(\mathrm{i} \omega_{n}\right)^{-4}\right)
$$

The results are

$$
\begin{aligned}
& \mathbf{C}_{\sigma \boldsymbol{K}^{\prime}}^{1}=\left(\begin{array}{ll}
1 & 0 \\
0 & 1
\end{array}\right) \\
& \mathbf{C}_{\sigma \boldsymbol{K}^{\prime}}^{2}=\left(\begin{array}{ll}
\bar{\xi}_{\boldsymbol{K}^{\prime}} & h_{\sigma} / 2 \\
h_{\sigma} / 2 & \bar{\xi}_{\boldsymbol{K}^{\prime}+\boldsymbol{Q}}
\end{array}\right)+\boldsymbol{\Sigma}_{\sigma \boldsymbol{K}^{\prime}}^{0}, \\
& \mathbf{C}_{\sigma \boldsymbol{K}^{\prime}}^{3}=\overline{\left[\left(\begin{array}{ll}
\xi_{\boldsymbol{K}^{\prime}} & h_{\sigma} / 2 \\
h_{\sigma} / 2 & \xi_{\boldsymbol{K}^{\prime}+\boldsymbol{Q}}
\end{array}\right)+\boldsymbol{\Sigma}_{\sigma \boldsymbol{K}^{\prime}}^{0}\right]^{2}}+\boldsymbol{\Sigma}_{\sigma \boldsymbol{K}^{\prime}}^{1},
\end{aligned}
$$

where the over-lined quantities are coarse-grained over the momentum patch centered around $\boldsymbol{K}^{\prime}$, e.g.

$$
\bar{\xi}_{\boldsymbol{K}^{\prime}}=\frac{1}{V} \int \mathrm{d} \tilde{\boldsymbol{k}} \xi_{\boldsymbol{K}^{\prime}+\tilde{\boldsymbol{k}}}
$$


A direct calculation of the Green function using Heisenberg's equations of motion provides the information necessary for the determination of the unknown coefficients $\boldsymbol{\Sigma}_{\sigma \boldsymbol{K}^{\prime}}^{0}$ and $\boldsymbol{\Sigma}_{\sigma \boldsymbol{K}^{\prime}}^{1}$. Starting again with the Hamiltonian eq. (3.37), the high-frequency coefficients of the single-particle Green function in real space

$$
G_{\sigma i j}\left(\mathrm{i} \omega_{n}\right)=\frac{C_{\sigma i j}^{1}}{\mathrm{i} \omega_{n}}+\frac{C_{\sigma i j}^{2}}{\left(\mathrm{i} \omega_{n}\right)^{2}}+\frac{C_{\sigma i j}^{3}}{\left(\mathrm{i} \omega_{n}\right)^{3}}+\mathcal{O}\left(\left(\mathrm{i} \omega_{n}\right)^{-4}\right)
$$

can be obtained [35] via

$$
\begin{aligned}
& C_{\sigma i j}^{1}=\left\langle\left\{c_{i \sigma}, c_{j \sigma}^{\dagger}\right\}\right\rangle, \\
& C_{\sigma i j}^{2}=-\left\langle\left\{\left[H_{h}, c_{i \sigma}\right], c_{j \sigma}^{\dagger}\right\}\right\rangle, \\
& C_{\sigma i j}^{3}=\left\langle\left\{\left[H_{h},\left[H_{h}, c_{i \sigma}\right]\right], c_{j \sigma}^{\dagger}\right\}\right\rangle
\end{aligned}
$$

using the Hamiltonian eq. (3.37). Here $[A, B](\{A, B\})$ denotes the (anti)commutator of the operators $A$ and $B$. A straightforward calculation yields

$$
\begin{aligned}
C_{\sigma i j}^{1}= & \delta_{i j}, \\
C_{\sigma i j}^{2}= & -\tilde{\xi}_{i j}-\left[h_{\sigma i}+U\left\langle n_{\bar{\sigma} i}\right\rangle\right] \delta_{i j}, \\
C_{\sigma i j}^{3}= & \sum_{m} \tilde{\xi}_{i m} \tilde{\xi}_{m j}+\left(h_{\sigma i} h_{\sigma j}+U^{2}\left\langle n_{\bar{\sigma} i}\right\rangle\right) \delta_{i j} \\
& \quad-\left(h_{\sigma i}+h_{\sigma j}\right) \tilde{\xi}_{i j}-U\left(n_{\bar{\sigma} i}+n_{\bar{\sigma} j}\right)\left(\tilde{\xi}_{i j}-h_{\sigma i} \delta_{i j}\right),
\end{aligned}
$$

where $\tilde{\xi}_{i j}=t+\left(\mu+\frac{U}{2}\right) \delta_{i j}$ for nearest neighbors, $\tilde{\xi}_{i j}=t^{\prime}+\left(\mu+\frac{U}{2}\right) \delta_{i j}$ for nextnearest neighbors (if we consider next-nearest-neighbor hopping according to the Hamiltonian eq. (2.9)), and zero otherwise. The high-frequency coefficients of the coarse-grained Green function in cluster momentum space eq. (B.3) are readily calculated by a Fourier transformation of eqs. (B.12)-(B.14) followed by a coarse-graining in $\boldsymbol{k}$-space. One obtains

$$
\begin{aligned}
& \mathbf{C}_{\sigma \boldsymbol{K}^{\prime}}^{1}=\left(\begin{array}{ll}
1 & 0 \\
0 & 1
\end{array}\right), \\
& \mathbf{C}_{\sigma \boldsymbol{K}^{\prime}}^{2}=\left(\begin{array}{lc}
\overline{\tilde{\xi}}_{\boldsymbol{K}^{\prime}}+U\left\langle n_{\bar{\sigma}}\right\rangle & U\left\langle m_{\bar{\sigma}}\right\rangle+h_{\sigma} / 2 \\
U\left\langle m_{\bar{\sigma}}\right\rangle+h_{\sigma} / 2 & \overline{\tilde{\xi}}_{\boldsymbol{K}^{\prime}+\boldsymbol{Q}}+U\left\langle n_{\bar{\sigma}}\right\rangle
\end{array}\right), \\
& \mathbf{C}_{\sigma \boldsymbol{K}^{\prime}}^{3}=\left(\begin{array}{l}
\overline{\tilde{\xi}}^{2} \boldsymbol{K}^{\prime}+h_{\sigma}^{2} / 4+2 U\left\langle n_{\bar{\sigma}}\right\rangle \overline{\tilde{\xi}}_{\boldsymbol{K}^{\prime}}+U^{2}\left\langle n_{\bar{\sigma}}\right\rangle+U h_{\sigma}\left\langle m_{\bar{\sigma}}\right\rangle \\
\left(U\left\langle m_{\bar{\sigma}}\right\rangle+h_{\sigma} / 2\right)\left(\overline{\tilde{\xi}}_{\boldsymbol{K}^{\prime}}+\overline{\tilde{\xi}}_{\boldsymbol{K}^{\prime}+\boldsymbol{Q}}\right)+U^{2}\left\langle m_{\bar{\sigma}}\right\rangle+U h_{\sigma}\left\langle n_{\bar{\sigma}}\right\rangle
\end{array}\right. \\
& \left.\begin{array}{l}
\left.\left(U\left\langle m_{\bar{\sigma}}\right\rangle+h_{\sigma} / 2\right)\left(\overline{\tilde{\xi}}_{\boldsymbol{K}^{\prime}}+\overline{\tilde{\xi}}_{\boldsymbol{K}^{\prime}+\boldsymbol{Q}}\right)+U^{2}\left\langle m_{\bar{\sigma}}\right\rangle+U h_{\sigma}\left\langle n_{\bar{\sigma}}\right\rangle\right), \\
\overline{\tilde{\xi}}^{2} \boldsymbol{K}^{\prime}+\boldsymbol{Q} \\
+h_{\sigma}^{2} / 4+2 U\left\langle n_{\bar{\sigma}}\right\rangle \overline{\tilde{\xi}}_{\boldsymbol{K}^{\prime}+\boldsymbol{Q}}+U^{2}\left\langle n_{\bar{\sigma}}\right\rangle+U h_{\sigma}\left\langle m_{\bar{\sigma}}\right\rangle
\end{array}\right),
\end{aligned}
$$


where $\tilde{\xi}_{\boldsymbol{K}^{\prime}}=\xi_{\boldsymbol{K}^{\prime}}-U / 2$ and

$$
\left\langle m_{\sigma}\right\rangle=\sum_{i} \mathrm{e}^{\mathrm{i} \boldsymbol{Q} \cdot \boldsymbol{r}_{i}}\left\langle n_{\sigma i}-n_{\bar{\sigma} i}\right\rangle
$$

These formulas constitute the basis of the Green function measurement at high frequencies discussed in section 4.2.2 (see, for example, fig. 4.2). A comparison with eqs. (B.4)-(B.6) yields

$$
\begin{aligned}
& \boldsymbol{\Sigma}_{\boldsymbol{K}^{\prime}}^{0}=U\left(\begin{array}{ll}
\left\langle n_{\bar{\sigma}}\right\rangle-\frac{1}{2} & \left\langle m_{\bar{\sigma}}\right\rangle \\
\left\langle m_{\bar{\sigma}}\right\rangle & \left\langle n_{\bar{\sigma}}\right\rangle-\frac{1}{2}
\end{array}\right), \\
& \boldsymbol{\Sigma}_{\boldsymbol{K}^{\prime}}^{1}=U^{2}\left(\begin{array}{ll}
\left\langle n_{\bar{\sigma}}\right\rangle\left(1-\left\langle n_{\bar{\sigma}}\right\rangle\right)+\left\langle m_{\bar{\sigma}}\right\rangle^{2} & \left\langle m_{\bar{\sigma}}\right\rangle\left(1-2\left\langle n_{\bar{\sigma}}\right\rangle\right) \\
\left\langle m_{\bar{\sigma}}\right\rangle\left(1-2\left\langle n_{\bar{\sigma}}\right\rangle\right) & \left\langle n_{\bar{\sigma}}\right\rangle\left(1-\left\langle n_{\bar{\sigma}}\right\rangle\right)+\left\langle m_{\bar{\sigma}}\right\rangle^{2}
\end{array}\right),
\end{aligned}
$$

which is the solution shown in eq. (7.17). The non-diagonal parts vanish for $\left\langle m_{\sigma}\right\rangle=0$ and the expression simplifies to the paramagnetic solution eq. (7.3). 


\section{Appendix C}

\section{Sub-matrix updates for the CT-AUX algorithm}

Two numerical algorithmic improvements have significantly increased the size of systems accessible by simulations with the Hirsch-Fye algorithm: the "delayed" updates [4], and the "sub-matrix" updates [130]. The "delayed" update algorithm can be straightforwardly generalized to spin-flip operations in the CT-INT and CTAUX algorithms [195], and an adaptation of the concept of delayed updates to vertex insertion and removals in the interaction expansion was recently proposed as well [120]. Our implementation of the CT-AUX algorithm uses a generalization of the "sub-matrix" technique of ref. [130].

Both "delayed" and "sub-matrix" updates are based on efficient memory management. The central object in continuous-time algorithms is a matrix, which for large cluster calculations does not fit into the cache of computer memory. Monte Carlo updates often consist of rank one updates or matrix-vector products. Such updates perform $\mathcal{O}\left(k^{2}\right)$ operations on $\mathcal{O}\left(k^{2}\right)$ data, where $k$ is the average matrix size, and therefore run at the speed of memory. Matrix-matrix operations (with $O\left(k^{3}\right)$ operations executed on $O\left(k^{2}\right)$ data) could run at the speed of the registers, as more calculations as compared to memory access operations are performed.

This appendix chapter follows ref. [56] closely.

\section{C.1 Sub-matrix updates}

To derive the sub-matrix updates [130], we consider a step $n$ of the algorithm at which a spin at $p_{n}$ from $k$ interaction vertices is changed from $s_{p_{n}}$ to $s_{p_{n}}^{\prime}$. The new matrix $G^{n+1}$ is then given by eq. (4.53),

$$
\begin{aligned}
G_{i j}^{n+1} & =G_{i j}^{n}+\left(G_{i p_{n}}^{n}-\delta_{i p_{n}}\right) \lambda^{n} G_{p_{n} j}^{n}, \\
\lambda^{n} & =\mathrm{e}^{\gamma \operatorname{sgn}(\sigma)\left(s_{p_{n}}^{\prime}-s_{p_{n}}\right)}-1
\end{aligned}
$$


again omitting the dependence on spin $\sigma$ and expansion order $k$. A change to the inverse Green function matrix $A^{n}=\left(G^{n}\right)^{-1}$ is of the form [167]

$$
\begin{aligned}
A_{i j}^{n+1} & =A_{i j}^{n}+\gamma^{n}\left(A_{i p}^{n}-\delta_{i p}\right) \delta_{p j}, \\
\gamma^{n} & =e^{-\gamma \operatorname{sgn}(\sigma)\left(s_{p_{n}}^{\prime}-s_{p_{n}}\right)}-1 .
\end{aligned}
$$

This is commonly known as the Sherman-Morrison formula. We define $\tilde{A}_{i j}^{n}=A_{i j}^{n}+$ $\gamma^{n} A_{i p}^{n} \delta_{p j}$ with $\operatorname{det} \tilde{A}^{n}=\left(1+\gamma^{n}\right) \operatorname{det}\left(A^{n}\right)$. We then rewrite eq. (C.3) as $A_{i j}^{n+1}=$ $\tilde{A}_{i j}^{n}-\gamma^{n} \delta_{i p} \delta_{p j}$, and using $\operatorname{det}\left(A_{i j}+u_{i} v_{j}\right)=\left(1+v_{l}\left(A^{-1}\right)_{l q} u_{q}\right) \operatorname{det} A_{i j}$, we have

$$
\begin{aligned}
\operatorname{det} A^{n+1} & =\operatorname{det}\left(\tilde{A}^{n}\right) \operatorname{det}\left(1-\gamma^{n}\left[\left(\tilde{A}^{n}\right)^{-1}\right]_{p p}\right) \\
& =-\operatorname{det} A^{n} \gamma^{n}\left[G_{p p}^{n}-\frac{1+\gamma^{n}}{\gamma^{n}}\right] .
\end{aligned}
$$

This formula yields the determinant ratio $\operatorname{det} N^{n} / \operatorname{det} N^{n+1}=\operatorname{det} A^{n+1} / \operatorname{det} A^{n}$ needed for the acceptance or rejection of an update.

We can recursively apply eq. (C.5) to obtain an expression for performing multiple interaction changes, as long as they occur for different spins $p_{i} \neq p_{j}(i \neq j)$,

$$
\begin{aligned}
A_{i j}^{n+1} & =\tilde{A}_{i j}^{n}-\sum_{m=0}^{n} \gamma^{m} \delta_{i p_{m}} \delta_{p_{m} j} \\
& =\tilde{A}^{n}-X^{n}\left(Y^{n}\right)^{T},
\end{aligned}
$$

with $X_{i j}^{n}=\gamma^{j} \delta_{i p_{j}}$ and $\left(Y^{n}\right)_{i j}^{T}=\delta_{p_{i} j} . X$ and $Y^{T}$ are index matrices that label the changed spins and keep track of a prefactor $\gamma^{n}$.

For measurements, we need access to the Green function $G$. It is obtained after $n_{\max }$ steps by applying the Woodbury identity [199] to eq. (C.6),

$$
G^{q+1}=\tilde{G}+\tilde{G} X\left(1-Y^{T} \tilde{G} X\right)^{-1} Y^{T} \tilde{G}
$$

with $q$ denoting a Woodbury step combining $n_{\max }$ vertex update steps and where $\tilde{G}=\tilde{A}^{-1}$. After some simplification, eq. (C.7) can be shown to be

$$
G_{i j}^{q+1}=D_{i}^{-1}\left(G_{i j}-G_{i p_{n}} \Gamma_{p_{n} p_{m}}^{-1} G_{p_{m} j}\right) .
$$

Here we have introduced the $n_{\max } \times n_{\max }$ matrix $\Gamma$, defined as

$$
\Gamma_{p q}=G_{p q}^{0}-\delta_{p q} \frac{1+\gamma_{p}}{\gamma_{p}}
$$

and a vector $D$ that is 1 everywhere but at positions where auxiliary spins are changed,

$$
D_{p_{n}}^{-1}=\frac{1}{1+\gamma^{n}}
$$


Note that $G_{p q}^{0}$ is the interacting Green function at step $n=0$ and not the effective bare Green function.

Translating this Green function formalism to a formalism for $N$ is straightforward: writing $G=N G^{0}$ and multiplying eq. (C.8) from the right with $\left(G^{0}\right)^{-1}$ yields

$$
N_{i j}^{q+1}=D_{i}^{-1}\left(N_{i j}-G_{i p_{n}} \Gamma_{p_{n} p_{m}}^{-1} N_{p_{m} j}\right)
$$

where one $G_{i p_{n}}$ remains in eq. (C.11).

Inserting $G=N G_{0}$ into eq. (4.53) and setting $s^{\prime}=0\left(N^{\prime}=1\right)$ we obtain:

$$
\begin{aligned}
1 & =N e^{V}-N G_{0} e^{V}+N G_{0} \\
\left(N G_{0}\right)_{i j} & =\left(N_{i j} e^{V_{j}}-\delta_{i j}\right) /\left(e^{V_{j}}-1\right)=G_{i j} \\
N_{i j} & =G_{i j}\left(1-e^{-V_{j}}\right)+e^{-V_{j}} \delta_{i j} .
\end{aligned}
$$

The computation of $G$ from $N$ in this manner fails if the interaction is zero. In this case we need to compute $G_{i j}=N_{i k} G_{k j}^{0}$.

\section{C.2 Monte Carlo updates}

To either accept or reject a configuration change, we need to compute the determinant ratio $\operatorname{det} N^{n+1} / \operatorname{det} N^{n}$. Following ref. [130] we write

$$
\operatorname{det} A^{n+1}=(-1)^{n+1} \prod_{j=0}^{n} \gamma^{j} \operatorname{det} A^{0} \operatorname{det} \Gamma^{n} .
$$

We successively build $\Gamma^{n}$ by adding rows and columns while keeping track of a $L U$ decomposition of the matrix,

$$
\Gamma^{n}=\left(\begin{array}{cc}
\Gamma^{n-1} & s \\
w^{T} & d
\end{array}\right)=\left(\begin{array}{cc}
L^{n-1} & 0 \\
x^{T} & 1
\end{array}\right)\left(\begin{array}{cc}
U^{n-1} & y \\
0 & \beta
\end{array}\right)
$$

with $L y=s, U^{T} x=w$, and

$$
\beta=G_{p_{n} p_{n}}^{0}-\frac{1+\gamma^{n}}{\gamma^{n}}-x^{T} y
$$

where both $x^{T}$ and $y$ are computed in $O\left(n^{2}\right)$ by solving a linear equation for a triangular matrix. The determinant ratio needed for the acceptance of an update is

$$
\frac{\operatorname{det} A^{n+1}}{\operatorname{det} A^{n}}=-\beta \gamma^{n}
$$

These updates have been formulated for spins that have only been updated once. In the case where the same spin is changed twice or more, rows and columns in $\Gamma$, 
or $L$ and $U$, need to be modified. These changes are of the form $\Gamma \rightarrow \Gamma+u v^{T}$, and Bennett's algorithm [16] can be used to re-factorize the matrix.

To efficiently make use of the sub-matrix updates, we add an additional step before insertion and removal updates are performed. We insert a number $n_{\max }$ of randomly chosen non-interacting vertices with auxiliary spin $s=0$, which does not change the value of the partition function. Once these vertices are inserted, insertion and removal updates at the locations of the pre-inserted non-interacting vertices become identical to spin-flip updates.

To accommodate this pre-insertion step, we split our random walk into an inner and an outer loop. In the outer loop (labeled by q) we perform measurements of observables and run the preparation step discussed above as well as recompute steps. These steps are described in more detail below. In the inner loop (labeled by $n$ ) we perform $n_{\max }$ insertion, removal, or spin-flip updates at the locations of the pre-inserted non-interacting spins.

We begin a Monte Carlo sweep by generating randomly a set of $n_{\max }^{\text {ins }}$ pairs of (site, time) indices, where $n_{\max }^{\text {ins }}$ denotes the maximum insertions possible. We then compute the additional rows of the matrix $N$ for these non-interacting spins:

$$
\tilde{N}=\left(\begin{array}{ll}
N & 0 \\
\tilde{R} & 1
\end{array}\right)
$$

where $\tilde{R}$ is a matrix of size $k \times n_{\max }^{\text {ins }}$ containing the contributions of newly added non-interacting spins,

$$
\tilde{R}_{i j}=\mathcal{G}_{i n}^{0}\left(e^{-\gamma \sigma s_{n}}-1\right) N_{n j},
$$

as well as the Green function matrix $G=N \mathcal{G}^{0}$ for the new spins.

Vertex insertion updates are performed by proposing to flip one of the newly inserted non-interacting spins from value zero to either plus or minus one. The determinant ratio is obtained using eq. (C.17). Vertex removal updates consist accordingly of a proposed change of a spin form \pm 1 to zero. To perform a spin-flip update we choose a currently interacting spin with value \pm 1 and propose to flip it to $\mp 1$. Each time a update is accepted, the auxiliary spin is changed and the matrix $\Gamma$ is enlarged by a row and a column.

This scheme of insertion, removal, and spin-flip updates is repeated $n_{\max }$ times. With each accepted move the matrix $\Gamma$ grows by a row and a column. To keep the algorithm efficient we periodically recompute the full $N$-matrix using the Woodbury formula (C.11)

$$
N_{i j}^{q+1}=D_{i}^{-1}\left(G_{i j}-G_{i p_{n}} \Gamma_{p_{n} p_{m}}^{-1} N_{p_{m} j}\right),
$$

as $\Gamma$ grows with every accepted update, and the cost of computing determinant ratios is $O\left(n^{2}\right)$. Non-interacting auxiliary spins can then be removed from $N_{i j}^{q+1}$ by deleting the corresponding rows and columns. At the end of a sweep, if the system is thermalized, observable averages can be computed. 


\section{C.3 Summary}

For large problem sizes, the "sub-matrix" algorithm achieves a significant performance increase as compared to the traditional update scheme, by replacing the slow rank-one updates by faster matrix-matrix operations. Our implementation of the sub-matrix updates in the CT-AUX algorithm requires an additional preparation step in which non-interacting vertices with auxiliary spins $s=0$ are introduced. After this step, the CT-AUX vertex insertion and removal updates become equivalent to spin-flip updates. The sub-matrix algorithm then proceeds by manipulating the inverse of the Green function matrix, for which changes under auxiliary spin-flips are completely local. This allows for a significantly faster computation of the QMC transition probabilities under a spin-flip update. The algorithm keeps track of a number $k$ of these local changes, similar to the delayed update algorithm, and then performs a Green function update as a matrix-matrix multiply.

Because this algorithm requires additional overhead over the traditional CT-AUX implementation, there is an optimal choice for the maximum number of spin-flip updates $n_{\max }$ per Green function update which depends on problem size and architectural parameters such as the cache size. For an optimal value of $n_{\max }$, a speed increase up to a factor of 8 relative to the traditional CT-AUX updates can be found [56]. Without this increase in performance, the large-scale cluster calculations up to $N_{\mathrm{c}}=100$ necessary for the finite-size extrapolation in chapter 5 would not have been possible. 
Appendix C Sub-matrix updates for the CT-AUX algorithm 


\section{Appendix D}

\section{Analytic continuation: Implementation details}

This appendix chapter deals with algorithmic details of the MEM and SAI algorithms introduced in chapter 6. Our implementation of these algorithm is based on the libraries of the ALPS project [3, 9]. ALPS (Applications and Libraries for Physics Simulations) is an open source effort providing libraries and simulation codes for strongly correlated quantum-mechanical systems.

Both the maximum entropy method and stochastic analytical inference require a numerical treatment of the $\chi^{2}$ estimate eq. (6.6)

$$
\chi^{2}[A]=\sum_{n, m}\left(\bar{G}_{n}-G_{n}\right)^{*} C_{n m}^{-1}\left(\bar{G}_{m}-G_{m}\right)
$$

with (c.f. eq. (6.2))

$$
G_{n}=\int_{-\infty}^{\infty} \mathrm{d} \omega K_{n}(\omega) A(\omega)
$$

We apply a matrix-vector notation to eq. (D.1) obtaining

$$
\chi^{2}=(\overline{\boldsymbol{G}}-\boldsymbol{G})^{T} \mathbf{C}^{-1}(\overline{\boldsymbol{G}}-\boldsymbol{G})
$$

and

$$
\boldsymbol{G}=\int_{-\infty}^{\infty} \mathrm{d} \omega \tilde{\boldsymbol{K}}(\omega) A(\omega),
$$

where $\tilde{\boldsymbol{K}}(\omega)$ describes a vector with elements $K_{n}(\omega)$. Numerical diagonalization of the covariance matrix $\mathbf{C}$ yields the diagonal eigenvalue matrix $\mathbf{E}$ and a orthogonal transformation matrix $\mathbf{O}$ with $\mathbf{C}=\mathbf{O E O}^{T}$. We define $\boldsymbol{y}:=\mathbf{E}^{-1 / 2} \mathbf{O}^{T} \overline{\boldsymbol{G}}, \boldsymbol{K}:=$ $\mathbf{E}^{-1 / 2} \mathbf{O}^{T} \tilde{\boldsymbol{K}}$, and write

$$
\chi^{2}=\left|\boldsymbol{y}-\int_{-\infty}^{\infty} \mathrm{d} \omega \boldsymbol{K}(\omega) A(\omega)\right|^{2} .
$$




\section{D.1 Maximum entropy algorithm}

We specify an interval where the spectrum $A(\omega)$ is non-zero and discretize it on this interval using a grid of frequencies $\omega_{m}$. If we define the vector $\boldsymbol{a}$ with elements $a_{m}=A\left(\omega_{m}\right)\left(\omega_{m+1}-\omega_{m}\right)$ and the matrix $\mathbf{K}$ with elements $K_{n m}=K_{n}\left(\omega_{m}\right)$, the relation eq. (D.4) can be written as the matrix-vector product $\boldsymbol{G}=\mathbf{K} \boldsymbol{a}$, hence

$$
\chi^{2}=|\boldsymbol{y}-\mathbf{K} \boldsymbol{a}|^{2} .
$$

The central quantity of the maximum entropy method is the entropy eq. (6.7)

$$
S=-\sum_{m} a_{m} \ln \left(\frac{a_{m}}{d_{m}}\right)
$$

with respect to the default model $d_{m}=D\left(\omega_{m}\right)\left(\omega_{m}-\omega_{m+1}\right)$. The aim of the method is to minimize the quantity eq. (6.8)

$$
Q=\frac{1}{2} \chi^{2}-\alpha S
$$

with respect to $\boldsymbol{a}$ for several fixed values of $\alpha$. This can be performed by a nonlinear numerical optimization algorithm. We implemented a standard version of such an algorithm introduced by BRYAN [25]. The condition for an extremum of $Q$ is

$$
\nabla Q=\mathbf{K}^{T}(\boldsymbol{y}-\mathbf{K a})+\alpha \ln \left(\frac{\boldsymbol{a}}{\boldsymbol{d}}\right) \stackrel{!}{=} 0,
$$

where the expression $\ln \left(\frac{\boldsymbol{a}}{\boldsymbol{d}}\right)$ is understood as a vector with elements $\ln \left(a_{m} / d_{m}\right)$. The vector $\boldsymbol{a}$ has of order 100 elements depending on the chosen frequency grid. In order to avoid a search for a minimum in such a high number of dimensions a singularvalue decomposition of the matrix $\mathbf{K}$ is performed. The result is $\mathbf{K}=\tilde{\mathbf{U}} \tilde{\mathbf{\Sigma}} \tilde{\mathbf{V}}^{T}$ with the diagonal matrix $\tilde{\boldsymbol{\Sigma}}$ and orthogonal matrices $\tilde{\mathbf{U}}$ and $\tilde{\mathbf{V}}$. The diagonal elements of $\tilde{\Sigma}$ are non-negative although many of them will be zero or at least equal or smaller than the machine precision of a computer. The dimension of $\tilde{\boldsymbol{\Sigma}}$ is therefore truncated to a small number $s$ (usually less than 10) and only the largest $s$ values are kept. We denote the truncated matrix by $\boldsymbol{\Sigma}$ and truncate the orthogonal transformation matrices accordingly, yielding $\mathbf{K}=\mathbf{U} \boldsymbol{\Sigma} \mathbf{V}^{T}$. The condition eq. (D.9) then becomes

$$
\boldsymbol{\Sigma} \mathbf{U}^{T}(\boldsymbol{y}-\mathbf{K} \boldsymbol{a})+\alpha \mathbf{V}^{T} \ln \left(\frac{\boldsymbol{a}}{\boldsymbol{d}}\right) \stackrel{!}{=} 0 .
$$

Finally, we substitute $\boldsymbol{a}$ by the small vector $\boldsymbol{u}$ of dimension $s$,

$$
\boldsymbol{u}:=\mathbf{V}^{T} \ln \left(\frac{\boldsymbol{a}}{\boldsymbol{d}}\right) \quad \Rightarrow \quad \boldsymbol{a}(\boldsymbol{u})=\boldsymbol{d} \exp (\mathbf{V} \boldsymbol{u})
$$

yielding

$$
\boldsymbol{\Sigma} \mathbf{U}^{T}(\boldsymbol{y}-\mathbf{K a}(\boldsymbol{u}))+\alpha \boldsymbol{u} \stackrel{!}{=} 0
$$

This is a nonlinear root-searching problem in $s$ dimensions, which can be solved iteratively using a Levenberg-Marquard algorithm [25, 187]. 


\section{D.2 Stochastic analytical inference}

Our implementation of the Monte Carlo procedure needed for the SAI algorithm follows ref. [13] closely. The SAI substitutes the spectrum $A(\omega)$ by the dimensionless field $n(x)$ writing (c.f. eq. (6.25))

$$
\chi^{2}=\left|\boldsymbol{y}-\int_{0}^{1} \mathrm{~d} x \hat{\boldsymbol{K}}(x) n(x)\right|^{2}
$$

analogous to eq. (D.5). In contrast to the maximum entropy algorithm, the SAI does not discretize the frequency axis but represents the field as a substitution of a finite set of delta functions with weights $n_{m}$ at positions $x_{m}$,

$$
n_{\mathcal{C}}(x)=\sum_{m} n_{m} \delta\left(x-x_{m}\right)
$$

The choice of weights and positions constitutes a Monte Carlo configuration $\mathcal{C}=$ $\left\{n_{m}, x_{m}\right\}$. The weights $n_{m}$ are non-negative and satisfy $\sum_{m} n_{m}=1$. This representation renders $\chi^{2}$ as well as the measure $\mathcal{D}^{\prime} n(x)$ (cf. eq. (6.28)) computationally tractable,

$$
\begin{aligned}
\chi_{\mathcal{C}}^{2} & =\left|\boldsymbol{y}-\sum_{m} \hat{\boldsymbol{K}}\left(x_{m}\right) n_{m}\right|^{2}, \\
\mathcal{D}^{\prime} n_{\mathcal{C}}(x) & =\prod_{m} \int_{0}^{\infty} \mathrm{d} n_{m} \int_{0}^{1} \mathrm{~d} x_{m} \delta\left(\sum_{m} n_{m}-1\right) .
\end{aligned}
$$

We can propose a new configuration $\mathcal{C}^{\prime}$ by changing a subset $\Lambda$ of the delta functions,

$$
\begin{aligned}
& n_{m} \rightarrow n_{m}^{\prime}+\sum_{l \in \Lambda} \delta_{m l} \Delta n_{l}, \\
& x_{m} \rightarrow x_{m}^{\prime}+\sum_{l \in \Lambda} \delta_{m l} \Delta x_{l},
\end{aligned}
$$

which produces a new $\chi^{2}$ estimate

$$
\chi_{\mathcal{C}^{\prime}}^{2}=\left|\boldsymbol{y}-\sum_{m} \hat{\boldsymbol{K}}\left(x_{m}\right) n_{m}+\sum_{l \in \Lambda}\left[\hat{\boldsymbol{K}}\left(x_{l}\right) n_{l}-\hat{\boldsymbol{K}}\left(x_{l}^{\prime}\right) n_{l}^{\prime}\right]\right|^{2} .
$$

We implemented two kinds of Monte Carlo updates: delta function moves and weight shifts. For the former we propose a random coordinate shift $\Delta x$ for a randomly chosen delta function. The latter are proposed shifts of the weights $n_{m}$. These updates must conserve the norm of the field. This is most easily accomplished by 
randomly choosing two delta functions 1 and 2 and proposing the shift $n_{1} \rightarrow n_{1}-\Delta n$ and $n_{2} \rightarrow n_{2}+\Delta n$, where $\Delta n \in\left[0, n_{1}\right]$. The simulation becomes more efficient if one uses weight shifts that incorporate more than two delta functions and which conserve not only the norm but also higher moments

$$
M_{k}=\int_{0}^{1} \mathrm{~d} x n_{\mathcal{C}}(x) x^{k}=\sum_{m} n_{m} x_{m}^{k}
$$

of the field configuration $[157,13]$. If one chooses $k>2$ delta functions $\Lambda=$ $\left\{\lambda_{1}, \ldots, \lambda_{k}\right\}$ and defines the scale factors

$$
Q_{l}=\left\{\begin{array}{ll}
1 & ; l=l_{1} \\
\frac{\prod_{m \in \Lambda \backslash\left\{l_{1}\right\}}\left(x_{m}-x_{l_{1}}\right)}{\prod_{m \in \Lambda \backslash\{l\}}\left(x_{m-x_{l}}\right)} & ; \text { else }
\end{array},\right.
$$

the weight shifts

$$
n_{l}^{\prime}=n_{l}-s Q_{l}
$$

conserve the lowest $l-1$ moments. In order to ensure positivity for all $n_{l}^{\prime}, s$ is chosen from the interval

$$
\max _{l \in \Lambda^{-}} \frac{r_{l}}{Q_{l}}<s<\min _{l \in \Lambda^{+}} \frac{r_{l}}{Q_{l}},
$$

where $\Lambda^{-}:=\left\{l: Q_{l}<0\right\}$ and $\Lambda^{+}:=\left\{l: Q_{l}>0\right\}$. We implemented updates up to $m=5$.

Update proposals are accepted by the usual Metropolis acceptance probability

$$
a\left(\mathcal{C} \rightarrow \mathcal{C}^{\prime}\right)=\min \left(1, \exp \left[-\frac{1}{2 \alpha}\left(\chi_{\mathcal{C}}^{2}-\chi_{\mathcal{C}^{\prime}}^{2}\right)\right]\right) .
$$

The most important Monte Carlo measurement is the average field configuration $n(x)$. It is measured by repeatedly recording a histogram of the moving delta functions.

The free-energy landscape of the system in question has proven to be quite complex. Previous implementations of the algorithm [157, 13] used either simulated annealing [93] or parallel tempering $[175,108,112]$ to overcome convergence problems of the simulations. We implemented a parallel-tempering algorithm, where several simulations with different regularization parameters $\alpha_{p}$ run in parallel. The temperature profile is chosen logarithmically with a constant modification factor $f=\alpha_{p+1} / \alpha_{p}$. This causes no further simulation effort, since a calculation of average spectra for a wide range of $\alpha$ is necessary anyway. All simulations, however, need to run in parallel and are not independent of each other. Neighboring simulations $\alpha_{p}$ and $\alpha_{p+1}$ are allowed to swap their configurations with probability

$$
p=\min \left(1, \exp \left[\left(\frac{1}{2 \alpha_{p}}-\frac{1}{2 \alpha_{p+1}}\right)\left(\chi_{p}^{2}-\chi_{p+1}^{2}\right)\right]\right) \text {. }
$$


Thus, configurations at low temperatures have access to high temperatures, where they can move more easily in phase space. This ensures that low temperature configuration can overcome barriers in the free-energy landscape.

\section{D.2.1 Wang-Landau algorithm}

A particular problem in the SAI is that a numerical treatment of eq. (6.41) involves the calculation of the quantity

$$
Z=\int \mathcal{D}^{\prime} n \mathrm{e}^{-\frac{1}{2} \chi^{2} / \alpha}
$$

This is equivalent to calculating a partition function in a canonical ensemble at temperature $\alpha$. Standard Monte Carlo techniques are only able to calculate thermal expectation values but not the partition function itself. We use a Wang-Landau algorithm $[189,188]$ to generate the density of states $\rho(E)$ of the system. Once $\rho(E)$ is calculated, the partition function can be obtained by

$$
Z=\int \mathrm{d} E \rho(E) \mathrm{e}^{-E / \alpha}
$$

The Wang-Landau algorithm performs a random walk in energy space with probability

$$
p(E)=1 / \rho(E)
$$

using the usual Metropolis weights, i. e., a proposed move from an energy $E_{1}$ to $E_{2}$ is accepted with the probability

$$
a\left(E_{1} \rightarrow E_{2}\right)=\min \left(1, \frac{\rho\left(E_{1}\right)}{\rho\left(E_{2}\right)}\right)
$$

Such a random walk visits all possible energy values with equal probability. We record a histogram $H(E)$ during the simulation: each time an energy $E$ is visited, the corresponding entry $H(E)$ is incremented by 1 . A random walk subject to the probability eq. (D.28) would produce a completely flat histogram.

Since the density of states is unknown at the beginning of the simulation, one starts with an arbitrary starting value, e.g., $\rho(E)=1$. For each visited energy, one multiplies the density of states by an modification factor $f>1$. This modification ensures that the current energy will be frequented less often in the further course of the simulation. As a result, the energies are visited more evenly. When the histogram is reasonably flat, one resets the histogram and restarts the simulation with a new modification factor $f^{\prime}=\sqrt{f}$. The starting value of $f$ is usually taken to be Euler's constant and the procedure is repeated until $f$ is very close to 1 (16 times in our implementation). Each new iteration further refines the structure of 
$\rho(E)$ until it converges to the density of states of the system up to an unknown normalization factor.

In order to speed up the algorithm, it is advisable to divide the energy range of interest into several slightly overlapping smaller intervals. This offers parallelization possibilities and accelerates convergence. The density of states varies over many orders of magnitude (cf. fig. 6.3b for an example). It is therefore only possible to store its logarithm. It nevertheless provides a possibility to evaluate the partition function eq. (D.26) and hence the probability distribution eq. (6.41). 


\section{Appendix E}

\section{Cluster table}

The following table lists optimized finite-size clusters of the simple cubic lattice in three dimensions following the rules determined in ref. [21]. It is meant to be an extension to the clusters already published in ref. [92].

Only bipartite clusters with an even number of cluster sites $N_{\mathrm{c}}$ are listed. All vectors $\boldsymbol{l}_{1}, \boldsymbol{l}_{2}, \boldsymbol{l}_{3}$ with individual components up to \pm 9 were examined by direct enumeration. The table shows the cluster with the smallest possible topological imperfection $I$, cubicity $c$ closest to one, and smallest surface area $A$, in this order of priority. If a cluster features only a marginally larger cubicity (less than $0.01 \%$ ) but a much smaller suface area (more than $20 \%$ ), the one with the smaller surface area is chosen instead. Not all cluster sizes are equally likely to produce high quality clusters (see Fig. 3.6). Thus, those cluster sizes are omitted where only relatively high imperfections and cubicities are possible.

Table E.1: Optimized bipartite clusters

\begin{tabular}{rcccrrr}
\hline$N_{\mathrm{c}}$ & $\boldsymbol{l}_{1}$ & $\boldsymbol{l}_{2}$ & $\boldsymbol{l}_{3}$ & $I$ & $c$ & $A$ \\
\hline 80 & $(4,1,-1)$ & $(-3,2,-3)$ & $(-2,3,3)$ & 4 & 1.05411 & 117.287 \\
96 & $(4,2,-2)$ & $(-4,2,-2)$ & $(-2,3,3)$ & 4 & 1.06462 & 131.417 \\
100 & $(4,2,-2)$ & $(-3,2,-3)$ & $(-2,3,3)$ & 6 & 1.02882 & 132.452 \\
156 & $(5,3,-2)$ & $(-4,2,-2)$ & $(2,4,4)$ & 11 & 1.04364 & 182.029 \\
158 & $(5,3,-2)$ & $(-4,1,-3)$ & $(-3,3,4)$ & 10 & 1.04959 & 184.262 \\
162 & $(5,2,-1)$ & $(-2,-4,-4)$ & $(-4,3,-3)$ & 8 & 1.06373 & 187.548 \\
168 & $(5,3,-2)$ & $(-4,0,-4)$ & $(-3,3,4)$ & 9 & 1.07053 & 197.177 \\
174 & $(5,2,-3)$ & $(-3,5,0)$ & $(3,4,3)$ & 10 & 1.06760 & 201.745 \\
180 & $(4,4,-2)$ & $(-4,-2,-4)$ & $(-3,4,3)$ & 11 & 1.05875 & 202.473 \\
188 & $(5,3,-2)$ & $(-2,4,-4)$ & $(-3,3,4)$ & 9 & 1.05157 & 207.287 \\
190 & $(5,3,-2)$ & $(-5,2,-3)$ & $(-3,3,4)$ & 10 & 1.06137 & 207.225 \\
194 & $(5,3,-2)$ & $(-2,-4,-4)$ & $(-4,3,-3)$ & 12 & 1.04167 & 207.776 \\
196 & $(5,3,-2)$ & $(-4,2,-4)$ & $(-3,3,4)$ & 13 & 1.03629 & 209.062 \\
198 & $(5,3,-2)$ & $(-4,3,-3)$ & $(2,3,5)$ & 14 & 1.04300 & 211.239 \\
\hline
\end{tabular}




\begin{tabular}{|c|c|c|c|c|c|c|}
\hline$N$ & $l_{1}$ & $\boldsymbol{l}_{2}$ & $l_{3}$ & $I$ & $c$ & $A$ \\
\hline 280 & $(6,4,-2)$ & $(-4,4,4)$ & $(4,1,5)$ & 14 & 1.06190 & 277.060 \\
\hline 282 & $(6,2,-2)$ & $(-5,3,-4)$ & $(-3,4,5)$ & 13 & .06070 & 72.050 \\
\hline 284 & $(6,4,-2)$ & $(-5,2,-3)$ & $(2,5,5)$ & 20 & 1.05982 & 272.817 \\
\hline 286 & $(5,5,-2)$ & $(-3,-2,-5)$ & $(-4,5,3)$ & 21 & 1.02963 & 272.601 \\
\hline 292 & $(6,3,-1)$ & $(-5,3,-4)$ & $(2,5,5)$ & 16 & 1.07416 & 78.630 \\
\hline 294 & $(6,2,-2)$ & $(-4,3,-5)$ & $(-3,4,5)$ & 19 & 1.04306 & 77.267 \\
\hline 298 & $(5,4,-3)$ & $(-4,-3,-5)$ & $(-4,5,-3)$ & 21 & 1.06352 & 287.151 \\
\hline 314 & $(5,5,-2)$ & $(-5,4,3)$ & $(3,-4,5)$ & 21 & 1.05739 & 295.808 \\
\hline 316 & $(6,4,-2)$ & $(-4,3,5)$ & $(4,-5,3)$ & 18 & 1.06232 & 6.746 \\
\hline 322 & $(5,5,-2)$ & $(-5,-2,-5)$ & $(-3,5,4)$ & 23 & 1.06622 & 299.074 \\
\hline 324 & $(6,4,-2)$ & $(-4,3,5)$ & $(3,-5,4)$ & 16 & 5347 & 99.188 \\
\hline 330 & $(6,3,-3)$ & $(-5,3,-4)$ & $(-3,4,5)$ & 19 & 217 & 96.425 \\
\hline 332 & $(6,4,-2)$ & $(-4,3,5)$ & $(2,-5,5)$ & 20 & 1.06057 & 304.019 \\
\hline 336 & $(6,5,-1)$ & $(-3,5,-4)$ & $(-5,3$ & 22 & & .831 \\
\hline 340 & $(7,3,-2)$ & $(-5,2,-5)$ & $(-4,4,4)$ & 24 & 1.06152 & 307.845 \\
\hline 342 & $(6,3,-3)$ & $(-3,-5,-4)$ & $(-5,5,-2)$ & 25 & 107 & 393 \\
\hline 412 & $(6,5,-3)$ & $(-5,4,5)$ & $(4,2,6)$ & 29 & 1.07416 & .612 \\
\hline 416 & $(7,3,-2)$ & $(-4,4$, & $(2,-6,4)$ & 31 & & \\
\hline 420 & $(7,4,-1)$ & $(-5,4,5)$ & $(2,-6,4)$ & 29 & & .607 \\
\hline 422 & $(7,3,-2)$ & $(-1,6,-5)$ & $(-4,5,5)$ & 28 & 1.06524 & 362.717 \\
\hline 424 & $(5,5,-4)$ & $(-6,-2,-4)$ & $(-3,5,6)$ & 27 & 112 & .802 \\
\hline 430 & $(7,5,-2)$ & $(-5,4,3)$ & $(4,3,7)$ & 32 & 1.06523 & 376.913 \\
\hline 444 & $(7,5,-2)$ & $(-5,5,4)$ & $(4,2,6)$ & 21 & 1.06078 & .755 \\
\hline 448 & $(6,5,-3)$ & $(-4,4,6)$ & $(6,1,5)$ & 27 & 72 & 156 \\
\hline 452 & $(7,4,-3)$ & $(-6,2,-4)$ & & 25 & & 934 \\
\hline 460 & $(7,4,-3)$ & $(-4,5,5)$ & $(6,-2,4)$ & 29 & 1.04672 & 371.302 \\
\hline 462 & $(5,5,-4)$ & $(-6,3,-3)$ & $(3,4,7)$ & 32 & 1.03192 & 372.631 \\
\hline 464 & $(7,3,-2)$ & $(-6,4,-4)$ & $(3,5,6)$ & 25 & 1.06330 & 375.665 \\
\hline 474 & $(7,4,-3)$ & $(-4,5,5)$ & $(4,1,7)$ & 26 & 1.06484 & 392.977 \\
\hline 484 & $(5,5,-4)$ & $(-6,3,-5)$ & $(-2,2,8)$ & 29 & 1.06773 & 392.684 \\
\hline 490 & $(6,5,-3)$ & $(-5,-3,-6)$ & $(-4,6,4)$ & 26 & 1.06179 & 397.412 \\
\hline 492 & $(7,4,-3)$ & $(-5,6,-3)$ & $(-4,4,6)$ & 27 & 1.07046 & 400.262 \\
\hline 498 & $(7,4,-3)$ & $(-4,5,5)$ & $(3,-6,5)$ & 26 & 1.05840 & 402.976 \\
\hline 500 & $(7,4,-3)$ & $(-4,4,6)$ & $(4,-6,4)$ & 25 & 1.05837 & 401.711 \\
\hline 504 & $(6,6,-2)$ & $(-5,4,5)$ & $(3,-6,5)$ & 29 & 1.05957 & 405.711 \\
\hline 508 & $(7,4,-3)$ & $(-3,6,-5)$ & $(-4,4,6)$ & 23 & 1.05849 & 405.394 \\
\hline 514 & $(7,5,-2)$ & $(-3,6,-5)$ & $(-5,4,5)$ & 26 & 1.05569 & 410.347 \\
\hline 516 & $(7,4,-3)$ & $(-4,4,6)$ & $(2,-6,6)$ & 27 & 1.06906 & 411.256 \\
\hline 518 & $(7,4,-3)$ & $(-4,4,6)$ & $(7,-3,4)$ & 32 & 1.06550 & 406.077 \\
\hline 520 & $(7,5,-2)$ & $(-5,3,6)$ & $(4,-6,4)$ & 33 & 1.05866 & 412.599 \\
\hline
\end{tabular}




\begin{tabular}{|c|c|c|c|c|c|c|}
\hline$N$ & $l_{1}$ & $l_{2}$ & $l_{3}$ & $I$ & C & $A$ \\
\hline 522 & $(7,4,-3)$ & $(-4,4,6)$ & $(3,-5,6)$ & 38 & 1.04971 & 406.186 \\
\hline 524 & $(8,4,-2)$ & $(-5,4,5)$ & $(3,-6,5)$ & 31 & 05959 & 7.563 \\
\hline 530 & $(7,5,-2)$ & $(-3,6,-5)$ & $(-5,3,6)$ & 38 & 1.05838 & 415.893 \\
\hline 532 & $(7,4,-3)$ & $(-7,4,-3)$ & $(-3,6,5)$ & 39 & 1.06024 & 412.773 \\
\hline 534 & $(7,5,-2)$ & $(-5,-2,-7)$ & $(-5,5,4)$ & 36 & 1.06245 & 423.184 \\
\hline 614 & $(7,6,-3)$ & $(-6,-3,-7)$ & $(-4,5,5)$ & 45 & 1.07069 & 75.127 \\
\hline 620 & $(6,5,-5)$ & $(-5,4,-7)$ & $(3,7,4)$ & 42 & 1.06942 & 474.396 \\
\hline 624 & $(8,3,-3)$ & $(-6,3,-5)$ & $(-4,6,6)$ & 44 & 1.04862 & 456.226 \\
\hline 626 & $(7,6,-3)$ & $(-6,5,5)$ & $(4,3,7)$ & 39 & 1.07238 & 81.495 \\
\hline 634 & $(8,4,-2)$ & $(-5,5,6)$ & $(2,-7,5)$ & 39 & 1.06262 & 474.309 \\
\hline 636 & $(6,5,-5)$ & $(-6,3,-7)$ & $(3,7,4)$ & 38 & 477 & 2.022 \\
\hline 638 & $(8,4,-2)$ & $(-4,3,7)$ & $(7,-5,4)$ & 45 & 967 & 7.337 \\
\hline 642 & $(7,6,-3)$ & $(-6,-2,-6)$ & $(-5,5,6)$ & 43 & & 35.276 \\
\hline 648 & $(8,5,-1)$ & $(-6,5,5)$ & $(2,-7,5)$ & 40 & & .139 \\
\hline 652 & $(6,6,-4)$ & $(-7,-2,-5)$ & $(-4,5,7)$ & 38 & 1.06647 & 487.652 \\
\hline 656 & $(7,2,-5)$ & $(-3,6,-7)$ & $(6,5$ & 36 & 82 & .656 \\
\hline 662 & $(8,4,-2)$ & $(-5,5,6)$ & $(6,-1,7)$ & 45 & 1.06521 & 487.957 \\
\hline 676 & $(8,5,-3)$ & $(-5,-2,-7)$ & $(-5,6,5)$ & 30 & 269 & .414 \\
\hline 678 & $(7,5,-4)$ & $(-4,7,-5)$ & $(-3,4,7)$ & 47 & 668 & 7.185 \\
\hline 682 & $(7,6,-1)$ & & & 35 & & .196 \\
\hline 686 & $(7,5,-4)$ & $(-5,7,-4)$ & $(-3,4,7)$ & 45 & 34 & 490.134 \\
\hline 696 & $(8,3,-3)$ & $(-4,-6,-6)$ & $(-7,5,-4)$ & 36 & 1.05831 & 491.571 \\
\hline 706 & $(8,5,-3)$ & $(-4,-2,-8)$ & $(-5,6,5)$ & 39 & 6161 & 512.003 \\
\hline 712 & $(8,5,-1)$ & $(-3,8,-5)$ & $(-6,5)$ & 36 & 7238 & 521.215 \\
\hline 718 & $(7,6,-3)$ & $(-5,5,6)$ & $(4,-7,5)$ & 39 & 1.06322 & .114 \\
\hline 726 & $(7,5,-4)$ & $(-5,-4,-7)$ & $(-5,7,4)$ & 37 & 1.06 & 517.827 \\
\hline 732 & $(7,6,-3)$ & $(-6,-3,-7)$ & $(-5,6,5)$ & 38 & 1.06 & 522.523 \\
\hline 740 & $(7,6,-3)$ & $(-5,4,7)$ & $(5,-7,4)$ & 46 & 099 & 525.370 \\
\hline 742 & $(8,5,-3)$ & $(-5,6,5)$ & $(3,-7,6)$ & 39 & 1.06576 & 531.178 \\
\hline 746 & $(9,5,-2)$ & $(-6,5,5)$ & $(4,-7,5)$ & 47 & 1.07101 & 538.668 \\
\hline 748 & $(7,6,-3)$ & $(-6,-3,-7)$ & $(-4,7,5)$ & 50 & 1.06724 & 527.466 \\
\hline 750 & $(8,5,-3)$ & $(-5,4,7)$ & $(5,-7,4)$ & 43 & 1.06411 & 529.209 \\
\hline 756 & $(8,5,-3)$ & $(-5,5,6)$ & $(3,-7,6)$ & 34 & 1.05986 & 531.951 \\
\hline 758 & $(7,7,-2)$ & $(-7,3,6)$ & $(5,-6,5)$ & 49 & 1.06599 & 536.797 \\
\hline 760 & $(8,5,-3)$ & $(-5,4,7)$ & $(4,-7,5)$ & 40 & 1.05948 & 531.445 \\
\hline 768 & $(8,6,-2)$ & $(-6,4,6)$ & $(4,-7,5)$ & 44 & 1.05945 & 539.047 \\
\hline 770 & $(8,5,-3)$ & $(-5,4,7)$ & $(3,-7,6)$ & 45 & 1.06387 & 535.917 \\
\hline 772 & $(8,6,-2)$ & $(-6,5,5)$ & $(3,-7,6)$ & 46 & 1.06094 & 542.929 \\
\hline 776 & $(9,5,-2)$ & $(-4,7,-5)$ & $(-6,4,6)$ & 48 & 1.06441 & 544.589 \\
\hline 780 & $(9,4,-3)$ & $(-7,3,-6)$ & $(-5,5,6)$ & 50 & 1.06570 & 536.537 \\
\hline
\end{tabular}




\begin{tabular}{rcccrrr}
\hline$N$ & $\boldsymbol{l}_{1}$ & $\boldsymbol{l}_{2}$ & $\boldsymbol{l}_{3}$ & $I$ & $c$ & $A$ \\
\hline 786 & $(9,5,-2)$ & $(-6,5,5)$ & $(3,-7,6)$ & 53 & 1.06183 & 551.440 \\
792 & $(8,6,-2)$ & $(-5,5,6)$ & $(3,-8,5)$ & 56 & 1.05955 & 550.741 \\
868 & $(8,6,-4)$ & $(-6,-4,-8)$ & $(-5,6,5)$ & 63 & 1.06979 & 599.507 \\
870 & $(8,6,-4)$ & $(-6,7,5)$ & $(5,4,7)$ & 62 & 1.06891 & 598.837 \\
872 & $(8,6,-2)$ & $(-6,7,5)$ & $(5,3,8)$ & 65 & 1.06962 & 593.299 \\
878 & $(8,7,-3)$ & $(-6,7,5)$ & $(5,4,7)$ & 62 & 1.07120 & 605.032 \\
882 & $(9,6,-3)$ & $(-6,5,5)$ & $(4,-8,6)$ & 60 & 1.07092 & 615.089 \\
896 & $(9,7,-2)$ & $(-6,6,4)$ & $(5,3,8)$ & 65 & 1.05459 & 601.062 \\
900 & $(8,6,-4)$ & $(-6,6,6)$ & $(5,3,8)$ & 51 & 1.07399 & 611.515 \\
902 & $(8,6,-4)$ & $(-6,-3,-7)$ & $(-5,7,6)$ & 58 & 1.06607 & 608.484 \\
904 & $(8,6,-4)$ & $(-4,-4,-8)$ & $(-6,7,5)$ & 57 & 1.06991 & 611.109 \\
906 & $(9,5,-4)$ & $(-6,-3,-7)$ & $(-6,7,5)$ & 56 & 1.07462 & 615.423 \\
910 & $(9,4,-3)$ & $(-5,6,7)$ & $(7,-3,6)$ & 58 & 1.05399 & 589.550 \\
912 & $(8,7,-3)$ & $(-7,6,5)$ & $(4,4,8)$ & 53 & 1.07406 & 620.929 \\
914 & $(9,4,-3)$ & $(-8,4,-6)$ & $(-4,5,7)$ & 64 & 1.04999 & 592.505 \\
918 & $(9,5,-2)$ & $(-6,5,7)$ & $(3,-8,5)$ & 54 & 1.06318 & 607.483 \\
920 & $(9,4,-3)$ & $(-5,6,7)$ & $(2,-8,6)$ & 53 & 1.06682 & 610.466 \\
924 & $(8,5,-3)$ & $(-6,5,7)$ & $(4,-8,6)$ & 51 & 1.06651 & 616.484 \\
926 & $(9,5,-4)$ & $(-6,8,4)$ & $(5,4,7)$ & 58 & 1.06259 & 621.652 \\
928 & $(9,5,-4)$ & $(-6,-4,-8)$ & $(-5,7,4)$ & 57 & 1.06462 & 620.966 \\
930 & $(8,7,-3)$ & $(-5,-3,-8)$ & $(-6,6,6)$ & 48 & 1.06837 & 623.034 \\
934 & $(9,6,-1)$ & $(-7,5,6)$ & $(3,-8,5)$ & 54 & 1.06720 & 620.966 \\
940 & $(8,5,-5)$ & $(-6,2,-8)$ & $(3,9,4)$ & 63 & 1.06641 & 614.538 \\
944 & $(8,7,-3)$ & $(-5,7,6)$ & $(5,3,8)$ & 61 & 1.06574 & 624.861 \\
948 & $(8,6,-2)$ & $(-7,5,6)$ & $(4,-8,6)$ & 47 & 1.07054 & 629.040 \\
950 & $(8,5,-5)$ & $(-7,-2,-7)$ & $(-4,7,7)$ & 62 & 1.06933 & 629.386 \\
952 & $(9,6,-1)$ & $(-2,8,-6)$ & $(-7,6,5)$ & 61 & 1.07195 & 632.337 \\
964 & $(8,7,-3)$ & $(-6,5,7)$ & $(6,2,8)$ & 55 & 1.07279 & 638.890 \\
966 & $(9,6,-3)$ & $(-6,7,5)$ & $(5,3,8)$ & 42 & 1.06310 & 635.469 \\
972 & $(9,6,-3)$ & $(-6,6,6)$ & $(6,2,8)$ & 39 & 1.07195 & 641.354 \\
984 & $(9,5,-4)$ & $(-5,7,6)$ & $(6,2,8)$ & 49 & 1.06520 & 641.604 \\
986 & $(9,7,-2)$ & $(-6,7,5)$ & $(5,3,8)$ & 52 & 1.06325 & 645.361 \\
988 & $(7,7,-4)$ & $(-7,6,-5)$ & $(5,5,8)$ & 55 & 1.07118 & 642.496 \\
994 & $(9,4,-3)$ & $(-4,-8,-6)$ & $(-8,5,-5)$ & 52 & 1.06831 & 630.719 \\
998 & $(9,5,-4)$ & $(-6,5,7)$ & $(5,-7,6)$ & 54 & 1.07303 & 646.831 \\
1000 & $(8,7,-3)$ & $(-6,8,4)$ & $(4,4,8)$ & 65 & 1.04999 & 637.212 \\
\hline & & & & & &
\end{tabular}




\section{Bibliography}

[1] A. A. Abrikosov, L. P. Gorkov, and I. E. Dzyaloshinski, Methods of quantum field theory in statisitical physics, pp. 130-143, Prentice Hall, New Jersey, 1963.

[2] H. Akima, A new method of interpolation and smooth curve fitting based on local procedures, J. ACM 17, 589-602 (1970).

[3] A. F. Albuquerque, F. Alet, P. Corboz, P. Dayal, A. Feiguin, S. Fuchs, L. Gamper, E. Gull, S. Gürtler, A. Honecker, R. Igarashi, M. Körner, A. Kozhevnikov, A. Läuchli, S. R. Manmana, M. Matsumoto, I. P. McCulloch, F. Michel, R. M. Noack, G. Pawłowski, L. Pollet, T. Pruschke, U. Schollwöck, S. Todo, S. Trebst, M. Troyer, P. Werner, and S. Wessel, The ALPS project release 1.3: Open-source software for strongly correlated systems, Journal of Magnetism and Magnetic Materials 310, 1187-1193 (2007).

[4] G. Alvarez, M. S. Summers, D. E. Maxwell, M. Eisenbach, J. S. Meredith, J. M. Larkin, J. Levesque, T. A. Maier, P. R. C. Kent, E. F. D'Azevedo, and T. C. Schulthess, New algorithm to enable $400+$ TFlop/s sustained performance in simulations of disorder effects in high- $T_{c}$ superconductors, Proceedings of the 2008 ACM/IEEE conference on Supercomputing (Piscataway, NJ, USA), SC '08, IEEE Press, 2008, pp. 61:1-61:10.

[5] P. W. Anderson, The resonating valence bond state in $\mathrm{La}_{2} \mathrm{CuO} 4$ and superconductivity, Science 235, 1196-1198 (1987).

[6] F. F. Assaad and T. C. Lang, Diagrammatic determinantal quantum Monte Carlo methods: Projective schemes and applications to the Hubbard-Holstein model, Phys. Rev. B 76, 035116 (2007).

[7] R. Aster, B. Borchers, and C. Thurber, Parameter estimation and inverse problems, Elsevier Academic Press, 2004.

[8] G. G. Batrouni, V. Rousseau, R. T. Scalettar, M. Rigol, A. Muramatsu, P. J. H. Denteneer, and M. Troyer, Mott domains of bosons confined on optical lattices, Phys. Rev. Lett. 89, 117203 (2002).

[9] B. Bauer, L. D. Carr, H.G. Evertz, A. Feiguin, J. Freire, S. Fuchs, L. Gamper, J. Gukelberger, E. Gull, S. Guertler, A. Hehn, R. Igarashi, S.V. Isakov, D. Koop, P.N. Ma, P. Mates, H. Matsuo, O. Parcollet, G. Pawłowski, J.D. Picon, L. Pollet, E. Santos, V.W. Scarola, U. Schollwöck, C. Silva, B. Surer, S. Todo, S. Trebst, M. Troyer, M. L. Wall, P. Werner, and S. Wessel, The 
ALPS project release 2.0: open source software for strongly correlated systems, http://arxiv.org/abs/1101.2646, 2011.

[10] G. Baym, Self-consistent approximations in many-body systems, Phys. Rev. 127, 1391-1401 (1962).

[11] G. Baym and L. P. Kadanoff, Conservation laws and correlation functions, Phys. Rev. 124, 287-299 (1961).

[12] G. Baym and C. Pethick, Landau Fermi-liquid theory: Concepts and applications, Wiley-VCH Verlag GmbH, 2007.

[13] K. S. D. Beach, Identifying the maximum entropy method as a special limit of stochastic analytic continuation, http://arxiv.org/abs/cond-mat/0403055, 2004.

[14] B. B. Beard and U.-J. Wiese, Simulations of discrete quantum systems in continuous euclidean time, Phys. Rev. Lett. 77, 5130-5133 (1996).

[15] G. Beni, P. Pincus, and D. Hone, High-temperature thermodynamics of the strongly correlated Hubbard model at arbitrary electron density, Phys. Rev. B 8, 3389-3397 (1973).

[16] J. Bennett, Triangular factors of modified matrices, Numerische Mathematik 7, 217-221 (1965).

[17] A. L. Berger, V. J. Della Pietra, and S. A. Della Pietra, A maximum entropy approach to natural language processing, Comput. Linguist. 22, 39-71 (1996).

[18] D. D. Betts, H. Q. Lin, and J. S. Flynn, Improved finite-lattice estimates of the properties of two quantum spin models on the infinite square lattice, Can. J. Phys. 77, 353-369 (1999).

[19] D. D. Betts, S. Masui, N. Vats, and G. E. Stewart, Improved finite-lattice method for estimating the zero-temperature properties of two-dimensional lattice models, Can. J. Phys. 74, 54-64 (1996).

[20] D. D. Betts, J. Schulenburg, G. E. Stewart, J. Richter, and J. S. Flynn, Exact diagonalization of the $s=1 / 2$ Heisenberg antiferromagnet on finite bcc lattices to estimate properties on the infinite lattice, Journal of Physics A: Mathematical and General 31, 7685 (1998).

[21] D. D. Betts and G. E. Stewart, Estimation of zero-temperature properties of quantum spin systems on the simple cubic lattice via exact diagonalization on finite lattices, Can. J. Phys. 75, 47-66 (1997).

[22] R. Blankenbecler, D. J. Scalapino, and R. L. Sugar, Monte Carlo calculations of coupled boson-fermion systems. I, Phys. Rev. D 24, 2278-2286 (1981).

[23] I. Bloch, J. Dalibard, and W. Zwerger, Many-body physics with ultracold gases, Rev. Mod. Phys. 80, 885-964 (2008).

[24] C. J. Bolech, S. S. Kancharla, and G. Kotliar, Cellular dynamical mean-field theory for the one-dimensional extended Hubbard model, Phys. Rev. B 67, 075110 (2003). 
[25] R. K. Bryan, Maximum entropy analysis of oversampled data problems, Eur. Biophys. J. 18, 165-174 (1990).

[26] R. Bulla, Zero temperature metal-insulator transition in the infinitedimensional Hubbard model, Phys. Rev. Lett. 83, 136-139 (1999).

[27] R. Bulla, T. A. Costi, and T. Pruschke, Numerical renormalization group method for quantum impurity systems, Rev. Mod. Phys. 80, 395-450 (2008).

[28] R. Bulla, T. A. Costi, and D. Vollhardt, Finite-temperature numerical renormalization group study of the Mott transition, Phys. Rev. B 64, 045103 (2001).

[29] E. Burovski, E. Kozik, N. Prokof'ev, B. Svistunov, and M. Troyer, Critical temperature curve in BEC-BCS crossover, Phys. Rev. Lett. 101, 090402 (2008).

[30] M. Caffarel and W. Krauth, Exact diagonalization approach to correlated fermions in infinite dimensions: Mott transition and superconductivity, Phys. Rev. Lett. 72, 1545-1548 (1994).

[31] M. Campostrini and E. Vicari, Critical behavior and scaling in trapped systems, Phys. Rev. Lett. 102, 240601 (2009).

[32] M. Campostrini and E. Vicari, Trap-size scaling in confined-particle systems at quantum transitions, Phys. Rev. A 81, 023606 (2010).

[33] D. Ceperley, G. V. Chester, and M. H. Kalos, Monte Carlo simulation of a many-fermion study, Phys. Rev. B 16, 3081-3099 (1977).

[34] D. M. Ceperley, Path integrals in the theory of condensed helium, Rev. Mod. Phys. 67, 279-355 (1995).

[35] Armin Comanac, Dynamical mean field theory of correlated electron systems: New algorithms and applications to local observables, $\mathrm{PhD}$ thesis, Columbia University, New York, 2007.

[36] F. Dalfovo, S. Giorgini, L. P. Pitaevskii, and S. Stringari, Theory of BoseEinstein condensation in trapped gases, Rev. Mod. Phys. 71, 463-512 (1999).

[37] K. B. Davis, M. O. Mewes, M. R. Andrews, N. J. van Druten, D. S. Durfee, D. M. Kurn, and W. Ketterle, Bose-Einstein condensation in a gas of sodium atoms, Phys. Rev. Lett. 75, 3969-3973 (1995).

[38] L. De Leo, C. Kollath, A. Georges, M. Ferrero, and O. Parcollet, Trapping and cooling fermionic atoms into Mott and Néel states, Phys. Rev. Lett. 101, 210403 (2008).

[39] F. H. Essler, H. Frahm, F. Göhmann, A. Klümper, and V. E. Korepin, The one-dimensional Hubbard model, Cambridge University Press, 2005.

[40] H. G. Evertz, The loop algorithm, Advances in Physics 52, 1-66 (2003).

[41] H. G. Evertz, G. Lana, and M. Marcu, Cluster algorithm for vertex models, Phys. Rev. Lett. 70, 875-879 (1993).

[42] W. M. C. Foulkes, L. Mitas, R. J. Needs, and G. Rajagopal, Quantum Monte Carlo simulations of solids, Rev. Mod. Phys. 73, 33-83 (2001). 
[43] S. Fuchs, E. Gull, L. Pollet, E. Burovski, E. Kozik, T. Pruschke, and M. Troyer, Thermodynamics of the 3D Hubbard model on approaching the Néel transition, Phys. Rev. Lett. 106, 030401 (2011).

[44] S. Fuchs, E. Gull, M. Troyer, M. Jarrell, and T. Pruschke, Spectral properties of the three-dimensional Hubbard model, http://arxiv.org/abs/1012.5950, 2010.

[45] S. Fuchs, T. Pruschke, and M. Jarrell, Analytic continuation of quantum Monte Carlo data by stochastic analytical inference, Phys. Rev. E 81, 056701 (2010).

[46] Sebastian Fuchs, Quanten-Monte-Carlo-Simulationen von Spinketten und analytische Fortsetzung dynamischer Korrelationsfunktionen, diploma thesis, Georg-August Universität Göttingen, 2006.

[47] P. Fulde, Electron correlations in molecules and solids, Springer Series in SolidState Sciences, Springer Verlag, Berlin/Heidelberg/New York, 1991.

[48] A. Georges, G. Kotliar, W. Krauth, and M. J. Rozenberg, Dynamical meanfield theory of strongly correlated fermion systems and the limit of infinite dimensions, Rev. Mod. Phys. 68, 13 (1996).

[49] E. V. Gorelik, I. Titvinidze, W. Hofstetter, M. Snoek, and N. Blümer, Néel transition of lattice fermions in a harmonic trap: A real-space dynamic meanfield study, Phys. Rev. Lett. 105, 065301 (2010).

[50] J. Graf, G.-H. Gweon, K. McElroy, S. Y. Zhou, C. Jozwiak, E. Rotenberg, A. Bill, T. Sasagawa, H. Eisaki, S. Uchida, H. Takagi, D.-H. Lee, and A. Lanzara, Universal high energy anomaly in the angle-resolved photoemission spectra of high temperature superconductors: Possible evidence of spinon and holon branches, Phys. Rev. Lett. 98, 067004 (2007).

[51] M. Greiner, O. Mandel, T. Esslinger, T. W. Hänsch, and I. Bloch, Quantum phase transition from a superfluid to a Mott insulator in a gas of ultracold atoms, Nature 415, 39-44 (2002).

[52] R. Grimm, M. Weidemüller, and Y. B. Ovchinnikov, Optical dipole traps for neutral atoms, Advances In Atomic, Molecular, and Optical Physics (B. Bederson and H. Walther, eds.), Advances In Atomic, Molecular, and Optical Physics, vol. 42, Academic Press, 2000, pp. 95-170.

[53] J. E. Gubernatis, M. Jarrell, R. N. Silver, and D. S. Sivia, Quantum Monte Carlo simulations and maximum entropy: Dynamics from imaginary-time data, Phys. Rev. B 44, 6011-6029 (1991).

[54] E. Gull, M. Ferrero, O. Parcollet, A. Georges, and A. J. Millis, Momentumspace anisotropy and pseudogaps: A comparative cluster dynamical mean-field analysis of the doping-driven metal-insulator transition in the two-dimensional Hubbard model, Phys. Rev. B 82, 155101 (2010).

[55] E. Gull, O. Parcollet, P. Werner, and A. J. Millis, Momentum-sector-selective metal-insulator transition in the eight-site dynamical mean-field approximation to the Hubbard model in two dimensions, Phys. Rev. B 80, 245102 (2009). 
[56] E. Gull, P. Staar, S. Fuchs, P. Nukala, M. S. Summers, T. Pruschke, T. Schulthess, and T. Maier, Sub-matrix updates for the continuous-time auxiliary-field algorithm, http://arxiv.org/abs/1010.3690, accepted for publication in Phys. Rev. B, 2010.

[57] E. Gull, P. Werner, S. Fuchs, B. Surer, T. Pruschke, and M. Troyer, Continuous-time quantum Monte Carlo impurity solvers, accepted for publication in Computer Physics Communications, 2011.

[58] E. Gull, P. Werner, A. Millis, and M. Troyer, Performance analysis of continuous-time solvers for quantum impurity models, Phys. Rev. B 76, 235123 (2007).

[59] E. Gull, P. Werner, O. Parcollet, and M. Troyer, Continuous-time auxiliaryfield Monte Carlo for quantum impurity models, EPL (Europhysics Letters) 82, 57003 (2008).

[60] Emanuel Gull, Continuous-time quantum Monte Carlo algorithms for fermions, PhD thesis, ETH Zürich, 2008.

[61] S. F. Gull, Bayesian inductive inference and maximum entropy, MaximumEntropy and Bayesian Methods in Science and Engineering (G. J. Erickson and C. R. Smith, eds.), vol. 1, Kluwer Academic Press, Dordrecht, 1988, p. 53.

[62] S. F. Gull and J. Skilling, Maximum entropy method in image processing, Communications, Radar and Signal Processing, IEE Proceedings F 131, 646 -659 (1984).

[63] M. C. Gutzwiller, Effect of correlation on the ferromagnetism of transition metals, Phys. Rev. Lett. 10, 159 (1963).

[64] O. Haan, J.-U. Klaetke, and K.-H. Mütter, Ground-state staggered magnetization of the antiferromagnetic Heisenberg model, Phys. Rev. B 46, 5723-5726 (1992).

[65] M. Han, W. Hua, W. Xu, and Y. Gong, An integrated baseball digest system using maximum entropy method, Proceedings of the 10th ACM international conference on Multimedia (New York, NY, USA), MULTIMEDIA '02, ACM, 2002, pp. 347-350.

[66] K. Harada and N. Kawashima, Universal jump in the helicity modulus of the two-dimensional quantum xy model, Phys. Rev. B 55, R11949-R11952 (1997).

[67] W. K. Hastings, Monte Carlo sampling methods using Markov chains and their applications, Biometrika 57, 97-109 (1970).

[68] F. Hébert, G. G. Batrouni, R. T. Scalettar, G. Schmid, M. Troyer, and A. Dorneich, Quantum phase transitions in the two-dimensional hardcore boson model, Phys. Rev. B 65, 014513 (2001).

[69] R. W. Helmes, T. A. Costi, and A. Rosch, Mott transition of fermionic atoms in a three-dimensional optical trap, Phys. Rev. Lett. 100, 056403 (2008). 
[70] M. H. Hettler, M. Mukherjee, M. Jarrell, and H. R. Krishnamurthy, Dynamical cluster approximation: Nonlocal dynamics of correlated electron systems, Phys. Rev. B 61, 12739-12756 (2000).

[71] M. H. Hettler, A. N. Tahvildar-Zadeh, M. Jarrell, T. Pruschke, and H. R. Krishnamurthy, Nonlocal dynamical correlations of strongly interacting electron systems, Phys. Rev. B 58, R7475-R7479 (1998).

[72] A. C. Hewson, The Kondo Problem to Heavy Fermions, Cambridge University Press, 1997.

[73] J. E. Hirsch, Discrete Hubbard-Stratonovich transformation for fermion lattice models, Phys. Rev. B 28, 4059-4061 (1983).

[74] J. E. Hirsch, Erratum: Discrete Hubbard-Stratonovich transformation for fermion lattice models, Phys. Rev. B 29, 4159 (1984).

[75] J. E. Hirsch and R. M. Fye, Monte Carlo method for magnetic impurities in metals, Phys. Rev. Lett. 56, 2521-2524 (1986).

[76] J. Hubbard, Electron correlations in narrow energy bands, Proc. Roy. Soc. London A 276, 238 (1963).

[77] C. Huscroft, M. Jarrell, T. Maier, S. Moukouri, and A. N. Tahvildarzadeh, Pseudogaps in the 2D Hubbard model, Phys. Rev. Lett. 86, 139-142 (2001).

[78] M. Imada, A. Fujimori, and Y. Tokura, Metal-insulator transitions, Rev. Mod. Phys. 70, 1039-1263 (1998).

[79] D. Jaksch, C. Bruder, J. I. Cirac, C. W. Gardiner, and P. Zoller, Cold bosonic atoms in optical lattices, Phys. Rev. Lett. 81, 3108-3111 (1998).

[80] D. Jaksch and P. Zoller, The cold atom Hubbard toolbox, Annals of Physics 315, 52-79 (2005).

[81] M. Jarrell and O. Biham, Dynamical approach to analytic continuation of quantum Monte Carlo data, Phys. Rev. Lett. 63, 2504-2507 (1989).

[82] M. Jarrell, J. K. Freericks, and T. Pruschke, Optical conductivity of the infinite-dimensional Hubbard model, Phys. Rev. B 51, 11704-11711 (1995).

[83] M. Jarrell and J. E. Gubernatis, Bayesian inference and the analytic continuation of imaginary-time quantum Monte Carlo data, Phys. Rep. 269, 133 (1996).

[84] M. Jarrell, T. Maier, C. Huscroft, and S. Moukouri, Quantum Monte Carlo algorithm for nonlocal corrections to the dynamical mean-field approximation, Phys. Rev. B 64, 195130 (2001).

[85] M. Jarrell and T. Pruschke, Magnetic and dynamic properties of the Hubbard model in infinite dimensions, Zeitschrift für Physik B Condensed Matter 90, 187-194 (1993), 10.1007/BF02198153.

[86] E. T. Jaynes, Bayesian methods: General background, Maximum-Entropy and Bayesian Methods in Applied Statistics (J. H. Justice, ed.), Cambridge University Press, Cambridge, 1986, p. 1. 
[87] J. Joo and V. Oudovenko, Quantum Monte Carlo calculation of the finite temperature Mott-Hubbard transition, Phys. Rev. B 64, 193102 (2001).

[88] R. Jördens, N. Strohmaier, K. Gunter, H. Moritz, and T. Esslinger, A Mott insulator of fermionic atoms in an optical lattice, Nature 455, 204-207 (2008).

[89] R. Jördens, L. Tarruell, D. Greif, T. Uehlinger, N. Strohmaier, H. Moritz, T. Esslinger, L. De Leo, C. Kollath, A. Georges, V. Scarola, L. Pollet, E. Burovski, E. Kozik, and M. Troyer, Quantitative determination of temperature in the approach to magnetic order of ultracold fermions in an optical lattice, Phys. Rev. Lett. 104, 180401 (2010).

[90] A. P. Kampf and J. R. Schrieffer, Spectral function and photoemission spectra in antiferromagnetically correlated metals, Phys. Rev. B 42, 7967-7974 (1990).

[91] J. Kanamori, Electron correlation and ferromagnetism of transition metals, Prog. Theor. Phys. 30, 275 (1963).

[92] P. R. C. Kent, M. Jarrell, T. A. Maier, and T. Pruschke, Efficient calculation of the antiferromagnetic phase diagram of the three-dimensional Hubbard model, Phys. Rev. B 72, 060411 (2005).

[93] S. Kirkpatrick, C. D. Gelatt, and M. P. Vecchi, Optimization by simulated annealing, Science 220, pp. 671-680 (1983).

[94] K. H. Knuth, A. E. Abbas, R. D. Morris, and J. P. Castle, Bayesian inference and maximum entropy methods in science and engineering, American Institute of Physics (AIP), 2005.

[95] M. Köhl, H. Moritz, T. Stöferle, K. Günter, and T. Esslinger, Fermionic atoms in a three dimensional optical lattice: Observing Fermi surfaces, dynamics, and interactions, Phys. Rev. Lett. 94, 080403 (2005).

[96] G. Kotliar, S. Y. Savrasov, K. Haule, V. S. Oudovenko, O. Parcollet, and C. A. Marianetti, Electronic structure calculations with dynamical mean-field theory, Rev. Mod. Phys. 78, 865-951 (2006).

[97] G. Kotliar, S. Y. Savrasov, G. Pálsson, and G. Biroli, Cellular dynamical mean field approach to strongly correlated systems, Phys. Rev. Lett. 87, 186401 (2001).

[98] B. Kyung and A.-M. S. Tremblay, Mott transition, antiferromagnetism, and d-wave superconductivity in two-dimensional organic conductors, Phys. Rev. Lett. 97, 046402 (2006).

[99] D. P. Landau and K. Binder, A guide to Monte Carlo simulations in statistical physics, Cambridge University Press, 2000.

[100] P.-S., Marquis de Laplace, Essai philosophique sur les probabilités, pp. 1-2, Courcier, Paris, 1814.

[101] P. A. Lee, N. Nagaosa, and X.-G. Wen, Doping a Mott insulator: Physics of high-temperature superconductivity, Rev. Mod. Phys. 78, 17-85 (2006). 
[102] A. J. Leggett, Bose-Einstein condensation in the alkali gases: Some fundamental concepts, Rev. Mod. Phys. 73, 307-356 (2001).

[103] E. H. Lieb and F. Y. Wu, Absence of Mott transition in an exact solution of the short-range, one-band model in one dimension, Phys. Rev. Lett. 20, 1445-1448 (1968).

[104] E. H. Lieb and F. Y. Wu, The one-dimensional Hubbard model: a reminiscence, Physica A: Statistical Mechanics and its Applications 321, 1-27 (2003).

[105] A. K. Livesey and J. C. Brochon, Analyzing the distribution of decay constants in pulse-fluorimetry using the maximum entropy method, Biophysical Journal 52, 693-706 (1987).

[106] J. M. Luttinger and J. C. Ward, Ground-state energy of a many-fermion system. II, Phys. Rev. 118, 1417-1427 (1960).

[107] J. N. Lyness, T. Søverik, and P. Keast, Notes on integration and integer sublattices, Mathematics of Computation 56, 243-255 (1991).

[108] A. P. Lyubartsev, A. A. Martsinovski, S. V. Shevkunov, and P. N. VorontsovVel'yaminov, New approach to Monte Carlo calculation of the free energy: Method of expanded ensembles, J. Chem. Phys. 96, 1776 (1992).

[109] T. Maier, M. Jarrell, T. Pruschke, and M. H. Hettler, Quantum cluster theories, Rev. Mod. Phys. 77, 1027-1080 (2005).

[110] T. A. Maier and M. Jarrell, Comparison of two-quantum-cluster approximations, Phys. Rev. B 65, 041104 (2002).

[111] T. A. Maier, M. Jarrell, T. C. Schulthess, P. R. C. Kent, and J. B. White, Systematic study of d-wave superconductivity in the $2 D$ repulsive Hubbard model, Phys. Rev. Lett. 95, 237001 (2005).

[112] E. Marinari and G. Parisi, Simulated tempering: A new Monte Carlo scheme, EPL (Europhysics Letters) 19, 451 (1992).

[113] N. Masuhara, J. M. Doyle, J. C. Sandberg, D. Kleppner, T. J. Greytak, H. F. Hess, and G. P. Kochanski, Evaporative cooling of spin-polarized atomic hydrogen, Phys. Rev. Lett. 61, 935-938 (1988).

[114] W. L. McMillan, Ground state of liquid He $\mathrm{e}^{4}$, Phys. Rev. 138, A442-A451 (1965).

[115] D. B. McWhan, A. Menth, J. P. Remeika, W. F. Brinkman, and T. M. Rice, Metal-insulator transitions in pure and doped $V_{2} O_{3}$, Phys. Rev. B 7, 1920-1931 (1973).

[116] H. J. Metcalf and P. van der Straten, Laser cooling and trapping of neutral atoms, Wiley-VCH Verlag, 2007.

[117] N. Metropolis, A. W. Rosenbluth, M. N. Rosenbluth, A. H. Teller, and E. Teller, Equation of state calculations by fast computing machines, The Journal of Chemical Physics 21, 1087-1092 (1953). 
[118] W. Metzner and D. Vollhardt, Correlated lattice fermions in $d=\infty$ dimensions, Phys. Rev. Lett. 62, 324-327 (1989).

[119] K. Mikelsons, A. Macridin, and M. Jarrell, Relationship between Hirsch-Fye and weak-coupling diagrammatic quantum Monte Carlo methods, Phys. Rev. E 79, 057701 (2009).

[120] Karlis Mikelsons, Extensions of numerical methods for strongly correlated electron systems, PhD thesis, University of Cincinnati, 2009.

[121] R. G. Miller, The jackknife - a review, Biometrika 61, 1 (1974).

[122] G. Modugno, G. Roati, F. Riboli, F. Ferlaino, R. J. Brecha, and M. Inguscio, Collapse of a degenerate Fermi gas, Science 297, 2240-2243 (2002).

[123] G. Moeller, Q. Si, G. Kotliar, M. Rozenberg, and D. S. Fisher, Critical behavior near the Mott transition in the Hubbard model, Phys. Rev. Lett. 74, 2082-2085 (1995).

[124] N. F. Mott, The basis of the electron theory of metals, with special reference to the transition metals, Proceedings of the Physical Society. Section A 62, 416 (1949).

[125] S. Moukouri and M. Jarrell, Absence of a Slater transition in the twodimensional Hubbard model, Phys. Rev. Lett. 87, 167010 (2001).

[126] E. Müller-Hartmann, Correlated fermions on a lattice in high dimensions, Zeitschrift für Physik 74, 507-512 (1989).

[127] Y. Nagaoka, Ferromagnetism in a narrow, almost half-filled s band, Phys. Rev. 147, 392-405 (1966).

[128] R. Narayan and R. Nityananda, Maximum entropy image restoration in astronomy, Annual Review of Astronomy and Astrophysics 24, 127-170 (1986).

[129] J. W. Negele and H. Orland, Quantum many-particle systems, Addison-Wesley Publishing Company, 1988.

[130] P. K. V. V. Nukala, T. A. Maier, M. S. Summers, G. Alvarez, and T. C. Schulthess, Fast update algorithm for the quantum Monte Carlo simulation of the Hubbard model, Phys. Rev. B 80, 195111 (2009).

[131] T. Obermeier, T. Pruschke, and J. Keller, Ferromagnetism in the large-U Hubbard model, Phys. Rev. B 56, R8479-R8482 (1997).

[132] J. Oitmaa and D. D. Betts, The ground state of two quantum models of magnetism, Can. J. Phys. 56, 897-901 (1978).

[133] T. Paiva, R. Scalettar, M. Randeria, and N. Trivedi, Fermions in $2 D$ optical lattices: Temperature and entropy scales for observing antiferromagnetism and superfluidity, Phys. Rev. Lett. 104, 066406 (2010).

[134] A. Papoulis, Probability and statistics, p. 422, Prentice Hall, New York, 1990.

[135] O. Parcollet, G. Biroli, and G. Kotliar, Cluster dynamical mean field analysis of the Mott transition, Phys. Rev. Lett. 92, 226402 (2004). 
[136] H. Park, K. Haule, C. A. Marianetti, and G. Kotliar, Dynamical mean-field theory study of Nagaoka ferromagnetism, Phys. Rev. B 77, 035107 (2008).

[137] R. Peters and T. Pruschke, Half-filled Hubbard model on a Bethe lattice with next-nearest-neighbor hopping, Phys. Rev. B 79, 045108 (2009).

[138] R. Peters and T. Pruschke, Magnetic phase diagram of the hubbard model with next-nearest-neighbour hopping, New J. Phys. 11, 083022 (2009).

[139] S. J. Phillips, R. P. Anderson, and R. E. Schapire, Maximum entropy modeling of species geographic distributions, Ecological Modelling 190, 231-259 (2006).

[140] L. Pollet, N. V. Prokof'ev, and B. V. Svistunov, Criticality in trapped atomic systems, Phys. Rev. Lett. 104, 245705 (2010).

[141] M. Potthoff, T. Wegner, and W. Nolting, Interpolating self-energy of the infinite-dimensional Hubbard model: Modifying the iterative perturbation theory, Phys. Rev. B 55, 16132-16142 (1997).

[142] N. V. Prokof'ev and B. V. Svistunov, Polaron problem by diagrammatic quantum Monte Carlo, Phys. Rev. Lett. 81, 2514-2517 (1998).

[143] N. V. Prokof'ev, B. V. Svistunov, and I. S. Tupitsyn, Exact, complete, and universal continuous-time worldline Monte Carlo approach to the statistics of discrete quantum systems, JETP Sov. Phys. 87, 310 (1998).

[144] R. Staudt, M. Dzierzawa, and A. Muramatsu, Phase diagram of the threedimensional Hubbard model at half filling, Eur. Phys. J. B 17, 411-415 (2000).

[145] E. L. Raab, M. Prentiss, A. Cable, S. Chu, and D. E. Pritchard, Trapping of neutral sodium atoms with radiation pressure, Phys. Rev. Lett. 59, 2631-2634 (1987).

[146] M. Rigol and A. Muramatsu, Quantum Monte Carlo study of confined fermions in one-dimensional optical lattices, Phys. Rev. A 69, 053612 (2004).

[147] M. Rigol, A. Muramatsu, G. G. Batrouni, and R. T. Scalettar, Local quantum criticality in confined fermions on optical lattices, Phys. Rev. Lett. 91, 130403 (2003).

[148] S. Rombouts, K. Heyde, and N. Jachowicz, A discrete Hubbard-Stratonovich decomposition for general, fermionic two-body interactions, Physics Letters A 242, 271-276 (1998).

[149] S. M. A. Rombouts, K. Heyde, and N. Jachowicz, Quantum Monte Carlo method for fermions, free of discretization errors, Phys. Rev. Lett. 82, 41554159 (1999).

[150] M. J. Rozenberg, G. Kotliar, and X. Y. Zhang, Mott-Hubbard transition in infinite dimensions. II, Phys. Rev. B 49, 10181-10193 (1994).

[151] A. N. Rubtsov, Quantum Monte Carlo determinantal algorithm without Hubbard-Stratonovich transformation: a general consideration, http://arxiv.org/abs/cond-mat/0302228, 2003. 
[152] A. N. Rubtsov and A. I. Lichtenstein, Continuous-time quantum Monte Carlo method for fermions: Beyond auxiliary field framework, JETP Letters 80, 61-65 (2004).

[153] A. N. Rubtsov, V. V. Savkin, and A. I. Lichtenstein, Continuous-time quantum Monte Carlo method for fermions, Phys. Rev. B 72, 035122 (2005).

[154] G. Rushbrooke, G. Baker, and P. Wood, PhaseTransitions and Critical Phenomena (C. Domb and M. Green, eds.), vol. 3, Academic Press, New York, 1974

[155] M. Sakata and M. Sato, Accurate structure analysis by the maximum-entropy method, Acta Crystallographica Section A 46, 263-270 (1990).

[156] A. W. Sandvik, Critical temperature and the transition from quantum to classical order parameter fluctuations in the three-dimensional Heisenberg antiferromagnet, Phys. Rev. Lett. 80, 5196-5199 (1998).

[157] A. W. Sandvik, Stochastic method for analytic continuation of quantum Monte Carlo data, Phys. Rev. B 57, 10287-10290 (1998).

[158] A. W. Sandvik, Multichain mean-field theory of quasi-one-dimensional quantum spin systems, Phys. Rev. Lett. 83, 3069-3072 (1999).

[159] A. W. Sandvik, Classical percolation transition in the diluted two-dimensional $S=\frac{1}{2}$ Heisenberg antiferromagnet, Phys. Rev. B 66, 024418 (2002).

[160] A. W. Sandvik and J. Kurkijärvi, Quantum Monte Carlo simulation method for spin systems, Phys. Rev. B 43, 5950-5961 (1991).

[161] V. W. Scarola, L. Pollet, J. Oitmaa, and M. Troyer, Discerning incompressible and compressible phases of cold atoms in optical lattices, Phys. Rev. Lett. 102, 135302 (2009).

[162] G. Schmid, S. Todo, M. Troyer, and A. Dorneich, Finite-temperature phase diagram of hard-core bosons in two dimensions, Phys. Rev. Lett. 88, 167208 (2002).

[163] U. Schneider, L. Hackermuller, S. Will, T. Best, I. Bloch, T. A. Costi, R. W. Helmes, D. Rasch, and A. Rosch, Metallic and Insulating Phases of Repulsively Interacting Fermions in a 3D Optical Lattice, Science 322, 1520-1525 (2008).

[164] H. B. Schüttler and D. J. Scalapino, Monte Carlo studies of the dynamics of quantum many-body systems, Phys. Rev. Lett. 55, 1204-1207 (1985).

[165] P. Sengupta, A. W. Sandvik, and D. K. Campbell, Bond-order-wave phase and quantum phase transitions in the one-dimensional extended Hubbard model, Phys. Rev. B 65, 155113 (2002).

[166] J. W. Serene and D. W. Hess, Quasiparticle properties of the two-dimensional Hubbard model in a propagator-renormalized fluctuation-exchange approximation, Phys. Rev. B 44, 3391-3394 (1991). 
[167] J. Sherman and W. J. Morrison, Adjustment of an inverse matrix corresponding to a change in one element of a given matrix, The Annals of Mathematical Statistics 21, 124-127 (1950).

[168] J. Skilling, Maximum Entropy and Bayesian Methods (J. Skilling, ed.), Kluwer Academic Press, Dordrecht, 1989, p. 45.

[169] J. Skilling, Maximum Entropy and Bayesian Methods (J. Skilling, ed.), Kluwer Academic Press, Dordrecht, 1989, p. 53.

[170] J. C. Slater, Magnetic effects and the Hartree-Fock equation, Phys. Rev. 82, 538-541 (1951).

[171] M. Snoek, I. Titvinidze, C. Tőke, K. Byczuk, and W. Hofstetter, Antiferromagnetic order of strongly interacting fermions in a trap: real-space dynamical mean-field analysis, New Journal of Physics 10, 093008 (2008).

[172] G. E. Stewart, D. D. Betts, and J. S. Flynn, Extension of the method of exact diagonalization of quantum spin models to finite face centred cubic lattices and estimation of the $T=0$ properties of the $S=1 / 2 X Y$ ferromagnet on the infinite fcc lattice, Journal of the Physical Society of Japan 66, 3231-3236 (1997).

[173] N. Strohmaier, D. Greif, R. Jördens, L. Tarruell, H. Moritz, T. Esslinger, R. Sensarma, D. Pekker, E. Altman, and E. Demler, Observation of elastic doublon decay in the Fermi-Hubbard model, Phys. Rev. Lett. 104, 080401 (2010).

[174] M. Suzuki, Relationship between d-dimensional quantal spin systems and $(d+1)$-dimensional ising systems, Progress of Theoretical Physics 56, 14541469 (1976).

[175] R. H. Swendsen and J. Wang, Replica Monte Carlo simulation of spin-glasses, Phys. Rev. Lett. 57, 2607 (1986).

[176] O. F. Syljuåsen, Using the average spectrum method to extract dynamics from quantum Monte Carlo simulations, Phys. Rev. B 78, 174429 (2008).

[177] O. F. Syljuåsen and A. W. Sandvik, Quantum Monte Carlo with directed loops, Phys. Rev. E 66, 046701 (2002).

[178] T. Pruschke and R. Bulla, Hund's coupling and the metal-insulator transition in the two-band Hubbard model, Eur. Phys. J. B 44, 217-224 (2005).

[179] A. N. Tahvildar-Zadeh, J. K. Freericks, and M. Jarrell, Magnetic phase diagram of the Hubbard model in three dimensions: The second-order local approximation, Phys. Rev. B 55, 942-946 (1997).

[180] Jan M. Tomczak, Spectral and optical properties of correlated materials, PhD thesis, Ecole Polytechnique, Palaiseau, 2007.

[181] N.-H. Tong, S.-Q. Shen, and F.-C. Pu, Mott-Hubbard transition in infinite dimensions, Phys. Rev. B 64, 235109 (2001). 
[182] H. F. Trotter, On the product of semi-groups of operators, Proceedings of the American Mathematical Society 10, 545-551 (1959).

[183] S. Trotzky, Y.-A. Chen, U. Schnorrberger, P. Cheinet, and I. Bloch, Controlling and detecting spin correlations of ultracold atoms in optical lattices, Phys. Rev. Lett. 105, 265303 (2010).

[184] M. Troyer and U.-J. Wiese, Computational complexity and fundamental limitations to fermionic quantum Monte Carlo simulations, Phys. Rev. Lett. 94, 170201 (2005).

[185] P. G. J. van Dongen, Thermodynamics of the extended Hubbard model in high dimensions, Phys. Rev. Lett. 67, 757-760 (1991).

[186] W. T. Vetterling, B. P. Flannery, W. H. Press, and S. A. Teukolski, Numerical Recipes in Fortran 77 - Second Edition, ch. 2.7 - Inversion by Partitioning, p. 70, University Press, Cambridge, 1992.

[187] W. T. Vetterling, B. P. Flannery, W. H. Press, and S. A. Teukolski, Numerical Recipes in Fortran 77 - Second Edition, pp. 675-683, University Press, Cambridge, 1992.

[188] F. Wang and D. P. Landau, Determining the density of states for classical statistical models: A random walk algorithm to produce a flat histogram, Phys. Rev. E 64, 056101 (2001).

[189] F. Wang and D. P. Landau, Efficient, multiple-range random walk algorithm to calculate the density of states, Phys. Rev. Lett. 86, 2050-2053 (2001).

[190] X. Wang, E. Gull, L. de' Medici, M. Capone, and A. J. Millis, Antiferromagnetism and the gap of a Mott insulator: Results from analytic continuation of the self-energy, Phys. Rev. B 80, 045101 (2009).

[191] P. Weiss, Hypothesis of the molecular field and ferromagnetic properties, J. Phys. Radium 6, 661 (1907).

[192] A. Weiße and H. Fehske, Exact diagonalization techniques, Computational Many-Particle Physics (H. Fehske, R. Schneider, and A. Weie, eds.), Lecture Notes in Physics, vol. 739, Springer Berlin / Heidelberg, 2008, pp. 529-544.

[193] F. Werner, O. Parcollet, A. Georges, and S. R. Hassan, Interaction-induced adiabatic cooling and antiferromagnetism of cold fermions in optical lattices, Phys. Rev. Lett. 95, 056401 (2005).

[194] P. Werner, A. Comanac, L. de' Medici, M. Troyer, and A. J. Millis, Continuous-time solver for quantum impurity models, Phys. Rev. Lett. 97, 076405 (2006).

[195] P. Werner, E. Gull, O. Parcollet, and A. J. Millis, Momentum-selective metalinsulator transition in the two-dimensional Hubbard model: An 8-site dynamical cluster approximation study, Phys. Rev. B 80, 045120 (2009).

[196] P. Werner and A. J. Millis, Hybridization expansion impurity solver: General formulation and application to Kondo lattice and two-orbital models, Phys. Rev. B 74, 155107 (2006). 
[197] S. Wessel, F. Alet, M. Troyer, and G. G. Batrouni, Quantum Monte Carlo simulations of confined bosonic atoms in optical lattices, Phys. Rev. A 70, 053615 (2004).

[198] G. C. Wick, The evaluation of the collision matrix, Phys. Rev. 80, 268-272 (1950).

[199] M. A. Woodbury, Inverting modified matrices, Statistical Research Group, Memo. Rep. no. 42, Princeton University, Princeton, N. J., 1950.

[200] F. C. Zhang and T. M. Rice, Effective Hamiltonian for the superconducting Cu oxides, Phys. Rev. B 37, 3759-3761 (1988).

[201] R. Žitko, J. Bonča, and T. Pruschke, Van Hove singularities in the paramagnetic phase of the Hubbard model: DMFT study, Phys. Rev. B 80, 245112 (2009).

[202] R. Zitzler, T. Pruschke, and R. Bulla, Magnetism and phase separation in the ground state of the Hubbard model, The European Physical Journal B Condensed Matter and Complex Systems 27, 473-481 (2002). 


\section{Acknowledgements}

Zuerst möchte ich meinem Doktorvater Thomas Pruschke für seine hervorragende Betreuung danken, für gute Ideen und dafür, immer ein offenes Ohr für Fragen und Probleme gehabt zu haben.

Vielen Dank auch an meinen Korreferenten Fakher Assaad für seine Mühe und Bereitschaft sich mit meiner Arbeit zu beschäftigen.

Ein großer Dank an Emanuel Gull, ohne dessen Programm, Hilfe, Kontakte, Organisation und Zusammenarbeit die Doktorarbeit in dieser Form überhaupt nicht möglich gewesen wäre.

Many thanks to Mark Jarrell and his group for stimulating discussions and the kind hospitality during my stay in Cincinnati and Baton Rouge. I thank Karlis Mikelsons for providing a deeper understanding of cluster and quantum Monte Carlo techniques and for his indispensable help in overcoming many practical obstacles.

I would like to thank Lode Polet, Evgeny Kozik, and Evgeny Burovski for the fruitful collaboration, although we have not even met each other in person during the whole time we spent on the cold-atoms project.

I thank Matthias Troyer and the ALPS developers for providing a powerful library and for the chance to participate in their programming project.

Ein herzlicher Dank geht an meine Arbeitsgruppe, vor allem an meine Büromitbewohner Andreas Dirks und Stephan Filor für die motivierende Arbeitsatmosphäre, sowie an Oliver Bodensiek, Piet Dargel, Ansgar Kalz und Andreas Honecker für das hilfreiche Korrekturlesen dieser Arbeit.

Schließlich danke ich meinen Eltern für ihre Unterstützung und Förderung und natürlich meiner Frau Britta Fuchs für alles. 


\section{Curriculum vitae}

\section{Personal data}

Name: $\quad$ Sebastian Fuchs

Date of birth: September 28, 1980

Place of birth: Lippstadt, Germany

Nationality: German

\section{Education}

September 1992 Highschool, Niklas-Luhmann-Gymnasium Oerlinghausen, Ger- June 2000 many

June 5, 2000 Highschool degree (Abitur)

October 2001 University studies in physics, University of Göttingen, Ger- November 2006 many

November 9, University diploma, University of Göttingen, Advisor: Prof. 2006 Dr. Thomas Pruschke Thesis title: Quanten-Monte-Carlo-Simulationen von Spinketten und analytische Fortsetzung dynamischer Korrelationsfunktionen (Quantum Monte Carlo simulations of spin chains and analytic continuation of dynamic correlation functions)

December 2006 Doctoral studies, University of Göttingen

- January 2011

\section{Scholarships}

September 2003 University studies abroad, University of California, Santa Bar-

- May 2004 bara, USA, as exchange student of the University of California Education Abroad Program

January 2005 Scholarship, Studienstiftung des Deutschen Volkes (German - November 2006 National Academic Foundation)

October 2008 Research fellowship, University of Cincinnati, USA, as research

- December 2008 fellow of the German Academic Exchange Service (DAAD)

January 2009 Research fellowship, Louisiana State University, Baton Rouge, - March 2009 USA, as research fellow of the DAAD 


\section{Publication list}

1. A. F. Albuquerque, F. Alet, P. Corboz, P. Dayal, A. Feiguin, S. Fuchs, L. Gamper, E. Gull, S. Gürtler, A. Honecker, R. Igarashi, M. Körner, A. Kozhevnikov, A. Läuchli, S. R. Manmana, M. Matsumoto, I. P. McCulloch, F. Michel, R. M. Noack, G. Pawłowski, L. Pollet, T. Pruschke, U. Schollwöck, S. Todo, S. Trebst, M. Troyer, P. Werner, and S. Wessel, The ALPS project release 1.3: open source software for strongly correlated systems, Journal of Magnetism and Magnetic Materials 310, 1187 (2007)

2. S. Demeshko, G. Leibeling, S. Dechert, S. Fuchs, T. Pruschke, and F. Meyer, Alternating spin chains: Controlled assembly from bimetallic building blocks and QMC simulation of spin correlation, ChemPhysChem 8, 405 (2007)

3. F.-M. Nie, S. Demeshko, S. Fuchs, S. Dechert, T. Pruschke, and F. Meyer, Targeted self-assembly and quantum Monte Carlo magnetic study of an alternating nickel(II) $1 D$ coordination polymer composed of highly preorganized binuclear tectons, Dalton Transactions 30, 3971 (2008)

4. A. Kalz, A. Honecker, S. Fuchs, and T. Pruschke, Phase diagram of the Ising square lattice with competing interactions, European Physics Journal B 65, $533(2008)$

5. S. Fuchs, T. Pruschke, and M. Jarrell, Application of Bayesian inference to stochastic analytic continuation, Journal of Physics: Conference Series 200, 012041 (2010) (conference proceedings)

6. S. Fuchs, T. Pruschke, and M. Jarrell, Analytic continuation of quantum Monte Carlo data by stochastic analytical inference, Phys. Rev. E 81, 056701 (2010)

7. E. Gull, P. Werner, S. Fuchs, B. Surer, T. Pruschke, M. Troyer, ContinuousTime Quantum Monte Carlo Impurity Solvers, accepted for publication in Computer Physics Communications (2010)

8. S. Fuchs, E. Gull, L. Pollet, E. Burovski, E. Kozik, T. Pruschke, and M. Troyer, Thermodynamics of the 3D Hubbard model on approaching the Néel transition, Phys. Rev. Lett. 106, 030401 (2011)

9. E. Gull, P. Staar, S. Fuchs, P. Nukala, M. S. Summers, T. Pruschke, T. Schulthess, and T. Maier, Sub-matrix updates for the continuous-time auxiliary field algorithm, arXiv:1010.3690, accepted for publication in Phys. Rev. B (2010)

10. S. Fuchs, E. Gull, M. Troyer, M. Jarrell and T. Pruschke, Spectral properties of the three-dimensional Hubbard model, arXiv:1012.5950 (2010) 
11. B. Bauer, L. D. Carr, A. Feiguin, J. Freire, S. Fuchs, L. Gamper, J. Gukelberger, E. Gull, S. Guertler, A. Hehn, R. Igarashi, S. V. Isakov, D. Koop, P. N. Ma, P. Mates, H. Matsuo, O. Parcollet, G. Pawłowski, J. D. Picon, L. Pollet, E. Santos, V. W. Scarola, U. Schollwöck, C. Silva, B. Surer, S. Todo, S. Trebst, M. Troyer, M. L. Wall, P. Werner, S. Wessel The ALPS project release 2.0: Open source software for strongly correlated systems, arXiv:1101.2646 (2011)

12. A. Kalz, A. Honecker, S. Fuchs, and T. Pruschke, Quantum disordered ground state for hard-core bosons on the frustrated square lattice, arXiv:1101.5115 (2011) 